Florida International University FIU Digital Commons

FIU Electronic Theses and Dissertations

University Graduate School

$11-1-2013$

\title{
A Study on the Correlation of Bivariate And Trivariate Normal Models
}

Maria del Pilar Orjuela

Florida International University, pily.org@gmail.com

DOI: $10.25148 /$ etd.FI13120413

Follow this and additional works at: https:// digitalcommons.fiu.edu/etd

Part of the Multivariate Analysis Commons, and the Statistical Theory Commons

\section{Recommended Citation}

Orjuela, Maria del Pilar, "A Study on the Correlation of Bivariate And Trivariate Normal Models" (2013). FIU Electronic Theses and Dissertations. 976.

https://digitalcommons.fiu.edu/etd/976

This work is brought to you for free and open access by the University Graduate School at FIU Digital Commons. It has been accepted for inclusion in FIU Electronic Theses and Dissertations by an authorized administrator of FIU Digital Commons. For more information, please contact dcc@fiu.edu. 


\section{FLORIDA INTERNATIONAL UNIVERSITY \\ Miami, Florida}

\section{A STUDY ON THE CORRELATION OF BIVARIATE AND TRIVARIATE NORMAL MODELS}

A thesis submitted in partial fulfillment of the requirements for the degree of

MASTER OF SCIENCE

in STATISTICS

by

Maria del Pilar Orjuela Garavito 
To: Dean Kenneth Furton

College of Arts and Sciences

This thesis, written by Maria del Pilar Orjuela Garavito, and entitled A Study on the Correlation of Bivariate and Trivariate Normal Models, having been approved in respect to style and intellectual content, is referred to you for judgment.

We have read this thesis and recommend that it be approved.

$\begin{array}{r}\hline \text { Florence George } \\ \hline \text { Kai Huang, Co-Major Professor } \\ \hline \text { Jie Mi, Co-Major Professor }\end{array}$

Date of Defense: November 1, 2013

The thesis of Maria del Pilar Orjuela Garavito is approved.

\begin{tabular}{r} 
Dean Kenneth Furton \\
College of Arts and Sciences \\
\hline Dean Lakshmi N. Reddi \\
University Graduate School
\end{tabular}

Florida International University, 2013 
(C) Copyright 2013 by Maria del Pilar Orjuela Garavito

All rights reserved. 


\section{DEDICATION}

To my parents. 
ACKNOWLEDGMENTS

Thanks, everyone! 


\author{
ABSTRACT OF THE THESIS \\ A STUDY ON THE CORRELATION OF BIVARIATE AND TRIVARIATE \\ NORMAL MODELS \\ by \\ Maria del Pilar Orjuela Garavito \\ Florida International University, 2013 \\ Miami, Florida \\ Professor Jie Mi, Co-Major Professor \\ Professor Kai Huang, Co-Major Professor
}

Suppose two or more variables are jointly normally distributed. If there is a common relationship between these variables, it would be very important to quantify this relationship by a parameter called the correlation coefficient which measures its strength, and the use of it can develop an equation for predicting, and ultimately draw testable conclusion about the parent population.

My research focused on the correlation coefficient $\rho$ for the bivariate and trivariate normal distribution when equal variances and equal covariances are considered. Particularly, we derived the Maximum Likelihood Estimators (MLE) of the distribution parameters assuming all of them are unknown, and we studied the properties and asymptotic distribution of $\hat{\rho}$. Showing the asymptotic normality of the distribution of $\hat{\rho}$, we were able to construct confidence intervals of the correlation coefficient $\rho$ and test hypothesis about $\rho$. With a series of simulations, the performance of our new estimators were studied and were compared with those estimators that already exist in the literature. The results indicated that the MLE has a better or similar performance than the others.

Keywords: Bivariate Normal Distribution, Trivariate Normal Distribution, Correlation coefficient, MLE, Pearson Correlation Coefficient. 


\section{TABLE OF CONTENTS}

CHAPTER

PAGE

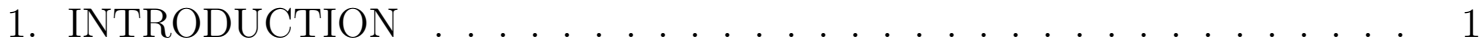

1.1 Motivation . . . . . . . . . . . . . . . . . . . . . 1

1.2 Background and Theory . . . . . . . . . . . . . . 2

2. BIVARIATE NORMAL DISTRIBUTION . . . . . . . . . . 5

2.1 General Discussion . . . . . . . . . . . . . . . . . . . . . . 5

2.2 Case of Equal Variances . . . . . . . . . . . . . . . . . . . . . . 7

2.2 .1 Data and Model . . . . . . . . . . . . . . . . . . 7

2.2 .2 Likelihood Function . . . . . . . . . . . . . . . . . . 7

2.2.3 Derivation of MLEs of Covariance Matrix Symmetric Bivariate Normal Parameters ................... 8

2.2.4 Properties of the MLEs of the Covariance Matrix Symmetric Bivariate Normal Parameters . . . . . . . . . . . . . . . . . . 11

2.2.5 Sampling Distributions of MLEs of the Covariance Matrix Symmetric Bivariate Normal Parameters . . . . . . . . . . . . . . 13

2.2.6 Discussion about the correlation coefficient $\hat{\rho} \ldots \ldots \ldots \ldots$. . . . . . . . 14

2.2.7 Variance Stabilizing transformation: The limiting distribution of the $z$ - transformation of $\hat{\rho} \ldots \ldots \ldots \ldots \ldots$

3. TRIVARIATE NORMAL DISTRIBUTION . . . . . . . . . . . . 26

3.1 General Discussion . . . . . . . . . . . . . . . . . . . . . . . 26

3.2 Covariance Matrix Symmetric . . . . . . . . . . . . . . . . . 27

3.2 .1 Model . . . . . . . . . . . . . . . . . . . . . . . 27

3.2 .2 Likelihood Function . . . . . . . . . . . . . . . . . . . . . . . 28

3.2.3 Derivation of Maximum Likelihood Estimators . . . . . . . . . . . . . . 29

3.2 .4 Properties of the MLE of $\rho \ldots \ldots \ldots \ldots$

3.3 Sampling Distribution of $\hat{\rho}$ of Trivariate Normal Distribution . . . . . . . 35

3.3 .1 The Limiting Distribution of $\hat{\rho} \ldots \ldots$. . . . . . . . . . 35

3.3.2 Variance-stabilizing transformation: The limiting distribution of the $z$ - transformation of $\hat{\rho} \ldots \ldots \ldots \ldots \ldots$

4. NUMERICAL STUDY ON THE CASE OF BIVARIATE NORMAL DIS-

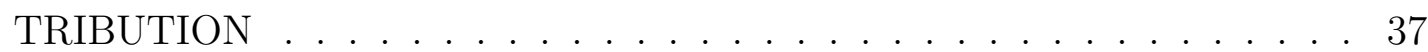

4.1 Comparison between $\hat{r}_{n}$ and $\hat{\rho}_{n} \ldots \ldots \ldots \ldots \ldots$

4.1.1 Comparison Point Estimators of the Correlation Coefficient $\rho$. . . . 38

4.1.2 Study on the Performance of the Estimators . . . . . . . . . . . . . . 41

4.2 Confidence Interval for $\rho \ldots \ldots \ldots$. . . . . . . . . . . 47

4.2.1 Study on the Denominator for the Confidence Intervals . . . . . . . . . 47

4.2 .2 Confidence Intervals . . . . . . . . . . . . . . . . . . . . 60

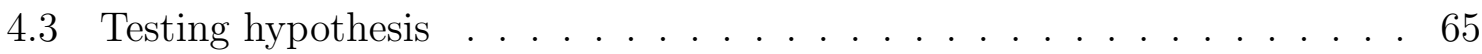


4.3.1 Probability of a Type I error . . . . . . . . . . . . . . . . 65

4.3.2 Power of the Test . . . . . . . . . . . . . . . . . . 67

5. NUMERICAL STUDY ON THE CASE OF TRIVARIATE NORMAL DIS-

TRIBUTION ............................. 76

5.1 Comparison Estimators of $\rho \ldots \ldots$. . . . . . . . . 76

5.1 .1 Point Estimators . . . . . . . . . . . . . . . . . . . 77

5.1.2 Study on the performance of the estimators . . . . . . . . . . . 79

5.2 Confidence Intervals . . . . . . . . . . . . . . . . . . . . . 84

5.2 .1 Study on the denominators . . . . . . . . . . . . . . . 85

5.2 .2 Confidence Intervals . . . . . . . . . . . . . . . . . . . . . 91

5.3 Test of Hypothesis . . . . . . . . . . . . . . . . . . . . . . . . . . . . . . 94

5.3.1 Probability Type I error . . . . . . . . . . . . . . . . . . . 94

5.3.2 Power of the test . . . . . . . . . . . . . . . . . . . . . . . . . . . . . . . . . . . . .

5.4 Conclusion . . . . . . . . . . . . . . . . . . . 102 


\section{LIST OF TABLES}

TABLE

PAGE

4.1 Comparison Point Estimators . . . . . . . . . . . . . . . . . 39

4.2 Comparison Bias Estimators . . . . . . . . . . . . . . . . . . 42

4.3 Comparison MSE Estimators . . . . . . . . . . . . . . . . 45

4.4 Comparison Denominadors MLE using Confidence Interval in Expres-

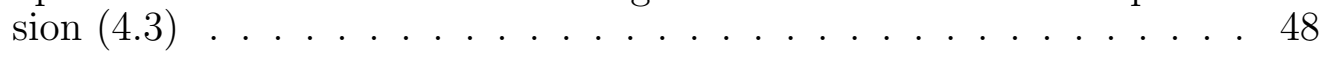

4.5 Cont. Comparison Denominadors MLE using Confidence Interval in

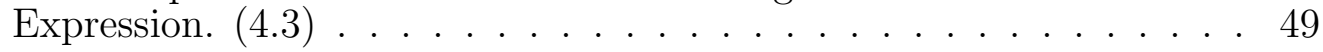

4.6 Comparison Denominadors Pearson using Confidence Interval in Expres-

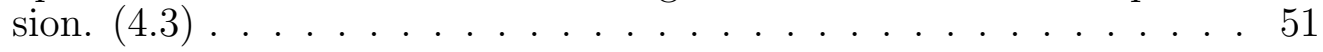

4.7 Cont. Comparison Denominadors Pearson using Confidence Interval in Expression. (4.3) . . . . . . . . . . . . . . 52

4.8 Comparison Denominadors MLE using Fisher Transformation . . . . . . 54

4.9 Cont. Comparison Denominadors MLE using Fisher Transformation . . 55

4.10 Comparison Denominadors Pearson using Fisher Dist. . . . . . . . . . . 57

4.11 Cont. Comparison Denominadors Pearson using Fisher Dist. . . . . . . . 58

4.12 Comparison Coverage Probability . . . . . . . . . . . . . . . 61

4.13 Comparison Average Width CI . . . . . . . . . . . . . 63

4.14 Comparison Type I Error . . . . . . . . . . . . . . . . . 66

4.15 Power MLE when $H a: \rho_{1}=\rho \pm 0.10 \ldots \ldots$. . . . . . . . . . . . 68

4.16 Power Pearson when $H a: \rho_{1}=\rho \pm .10$. . . . . . . . . . . . . . . . . 69

4.17 Power MLE when $H a: \rho_{1}=\rho \pm .05$. . . . . . . . . . . . . . . . . 72

4.18 Power Pearson when $\mathrm{Ha}: \rho_{1}=\rho \pm .05 \ldots$. . . . . . . . . . . . . 73

5.1 Comparison Point Estimators Trivariate . . . . . . . . . . . . 77

5.2 Comparison Bias of Estimators Trivariate . . . . . . . . . . . 80

5.3 Comparison MSE of Estimators Trivariate . . . . . . . . . . . . 82

5.4 Comparison Denominators MLE Using Normal Trivariate . . . . . . . . 86 
5.5 Comparison Denominators MLE Using Variance Stabilizing Transformation Trivariate . . . . . . . . . . . . . . . . . . . . . . 89

5.6 Coverage Probability Confidence Interval for $\rho$ Trivariate . . . . . . . . 91

5.7 Average Width of Confidence Interval for $\rho$ Trivariate . . . . . . . . 93

5.8 Type I error Trivariate . . . . . . . . . . . . . . . . . . . . . . . . 95

5.9 Comparison Power when $H a: \rho_{1}=\rho_{0} \pm 0.10$ using MLE Trivariate . . . 97

5.10 Comparison Power when $H a: \rho=\rho \pm 0.05$ using MLE Trivariate . . . 100 


\section{LIST OF FIGURES}

FIGURE

PAGE

4.1 Comparison Point Estimators of Rho . . . . . . . . . . . . . . 40

4.2 Comparison Bias of Point Estimators _ . . . . . . . . . . . . . . 43

4.3 Comparison Absolute Value of Bias . . . . . . . . . . . . . . . . 44

4.4 Comparison MSE . . . . . . . . . . . . . . . . 46

4.5 Comparison Denominators for MLE using Confidence Interval in Expression. $(4.3) \ldots \ldots \ldots \ldots \ldots \ldots \ldots$

4.6 Comparison Denominators for Pearson Estimator using Confidence Interval in Espression. (4.3) f . . . . . . . . . . . . . . 53

4.7 Comparison Denominators for MLE using Fisher Transformation . . . . 56

4.8 Comparison Denominators for Pearson Estimator using Fisher Trans-

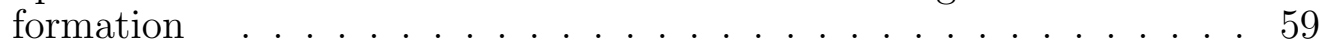

4.9 Comparison Coverage Probability Confidence Interval . . . . . . . . . . 62

4.10 Comparison Average Width of the 95\% Confidence Interval . . . . . . . 64

4.11 Comparison Type I error _ . . . . . . . . . . . . . . . . . . 67

4.12 Comparison Power $\mathrm{Ha}: \rho=\rho-0.10 \ldots \ldots \ldots$

4.13 Comparison Power $\mathrm{Ha}: \rho=\rho+0.10 \ldots \ldots \ldots \ldots$. . . . . . 71

4.14 Comparison Power $\mathrm{Ha}: \rho=\rho-0.05 \ldots \ldots \ldots \ldots$

4.15 Comparison Power Ha: $\rho=\rho+0.05 \ldots \ldots \ldots \ldots$

5.1 Comparison Point Estimators Trivariate Normal Distribution . . . . . 78

5.2 Comparison Bias Trivariate Normal Distribution . . . . . . . . . . . . 81

5.3 Comparison MSE Trivariate Normal Distribution _ . . . . . . . . . . 83

5.4 Comparison denominators using MLE in Expression $5.3 \ldots$. . . . . 87

5.5 Comparison denominator MLE using variance stabilizing transformation 90

5.6 Comparison Coverage Probability of CI using Fisher . . . . . . . . . . 92

5.7 Comparison Width 95\% CI using Fisher . . . . . . . . . . . . 94

5.8 Comparison Type I error _ . . . . . . . . . . . . . . . . . . . 96 


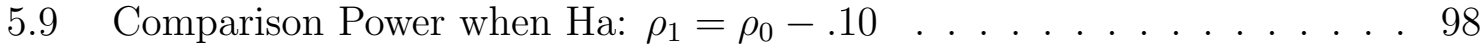

5.10 Comparison Power when $\mathrm{Ha}: \rho_{1}=\rho_{0}+.10$. . . . . . . . . . . . . . 99

5.11 Comparison Power when $\mathrm{Ha}: \rho_{1}=\rho_{0}-.05$. . . . . . . . . . . . . 101

5.12 Comparison Power when $H a: \rho_{1}=\rho_{0}+.05$. . . . . . . . . . . . . . . . 102 


\section{CHAPTER 1 \\ INTRODUCTION}

\subsection{Motivation}

The Normal Distribution is one of the most common distributions used in a variety of fields, not only because it appears in theoretical work as an approximation of the distribution of different kind of measurements, but also because there are so many phenomena in the nature that follows the normal distribution. For instance, in natural sciences, quantities such as height of people and intelligence coefficients are approximately normally distributed (Clauser [2007]); in physics velocities of the molecules in an ideal gas, the position of a particle which experiences diffusion and the ground state in a quantum harmonic oscillator, are also normally distributed.

The Multivariate case of the Normal Distribution and, in particular, the Bivariate and Trivariate cases, play a fundamental role in Multivariate Analysis. Bivariate normal distributions have shown to be important from the theoretical point of view and have been widely studied because its several useful and elegant properties. However, the Trivariate case, although also important, has received much less attention.

Sometimes there is a relationship and dependence among the variables under the Multivariate Normal Distribution. For this reason, would be very useful to quantify this relationship in a number called the correlation coefficient, measure its strength, develop an equation for predicting, and ultimately draw testable conclusion about the parent population. My thesis focuses on the measure of correlation, assuming that there is common relationship in hte Bivariate and Trivariate models when all the variables have common variance. 


\subsection{Background and Theory}

In the first half of the nineteenth century, scientists such as Laplace, Plana, Gauss and Bravais studied the Bivariate Normal and Seal and Lancaster contributed to these developments as well. But it was until 1888 when Francis Galton, who first introduced the concept of a measure of correlation in a bivariate data, re-described and recognized the mathematical definition of it and found some applications.

Inference regarding the correlation coefficient of two random variables from the Bivariate Normal Distribution has been investigated by many authors. In particular, Fisher (1915) and Hotelling (1953) derived exact forms of the density for the sample correlation coefficient but obtaining confidence intervals was computationally intensive (1988). Fisher also derived his famous transformation for large samples for the limiting distribution of the bivariate correlation coefficient, and, in 1921, he obtained results for the correlation coefficient for small samples (1921). Other authors such as Ruben (1966) established the distribution of the correlation coefficient in the form of a normalization approximation, and Kraemer (1973) and Samiuddin (1970) in the form of a $t$ approximations. More recently, Sun and Wong (2007) used a likelihood-based higher-order asymptotic method to obtain confidence intervals very accurate even when the sample size is small.

Some other authors, such as Goria (1980), Masry (2011) and Paul (1988) studied the correlation coefficient when specific assumptions are given to the Bivariate Normal distributions. For example, Goria (1980) deduced two test for testing the correlation coefficient in the bivariate normal population assuming that the ratio of the variances is known; Masry (2011) worked with the estimation of the correlation 
coefficient of bivariate data under dependence; and Paul (1988) proposed a method for testing the equality of several correlation coefficients, for more than two independent random samples from bivariate normal populations.

Since very little attempt, in the literature, has been made to develop and evaluate statistics for testing the validity of the assumption of a common correlation with equal variances, commonly called covariance permutation-symmetric for multivariate normal distribution, our task is then to explore and study the correlation coefficient under this assumption in the bivariate and trivariate normal distributions.

The idea of our study is that, on the basis of a random sample $\left(X_{1}, Y_{1}\right), \ldots,\left(X_{n}, Y_{n}\right)$ of $n$ observations from a Bivariate Normal population with equal variance, we will find the estimators of the parameters assuming that all of them are unknown. Particularly, we will derive the Maximum Likelihood estimators of the distribution parameters, and we will study the asymptotic distribution of correlation coefficient. Showing the asymptotic normality of this distribution, we will then be able to construct confidence intervals of it and test hypothesis. The performance of our new estimator will be studied and will be compared with those estimators that already exist in the literature.

In the case of the Trivariate Normal distribution, we derive the maximum likelihood estimator for the correlation coefficient from a random sample of $\mathrm{n}$ observations $\left(X_{11}, X_{21}, X_{31}\right), \ldots,\left(X_{n 1}, X_{n 1}, X_{n 1}\right)$ as well assuming that this sample comes from a covariance permutation-symmetric Trivariate Normal population. We will compare it with that one obtained from the average of pairwise bivariate Pearson correlation coefficients and the average of pairwise MLE of $\rho$. 
The thesis is organized as follows. The derivation of the Maximum Likelihood Estimators of the parameters of the Bivariate Normal distribution under the assumption of equal variances is reviewed in Chapter 2. Moreover, a discussion about the correlation coefficient is performed, whose properties are announced and sampling distribution is found. In Chapter 3 a similar procedure is made when a Trivariate Normal distribution is considered under the same assumption of equal variances. Chapter 4 and 5 focus on numerical computations using the MLE of the correlation coefficient for Bivariate and Trivariate cases respectively, comparing the performance of confidence regions, probability of Type I error and Power of testing for its true value. 


\section{CHAPTER 2}

\section{BIVARIATE NORMAL DISTRIBUTION}

In Chapter 2, we study a particular case of the Bivariate Normal Distribution. Using a frequently used method, namely the method of the Maximum Likelihood Estimation, we find the estimators of the parameters of this special case. We discuss its properties and focus on the MLE estimator of its correlation coefficient.

The outline of the chapter is as follows. In Section 2.1 we introduce the probability density function of the Bivariate Normal Distribution and its MLEs. In Section 2.2 we discuss the probability density function of the Bivariate Normal Distributionn with equal variances. In particular, in Section 2.2.2 we find the likelihood function and in Section 2.2.3 we discuss the MLEs of the parameters. Later, in Section 2.2.4 we show properties of those MLEs found before and finally, in Section 2.2.6, we focus on the study of the estimator of the correlation coefficient $\hat{\rho}$.

\subsection{General Discussion}

Let $X$ and $Y$ be two random variables. It is said that $(X, Y)$ have a Bivariate Normal Distribution if the joint probability density function $f(x, y)$ is of the form

$$
f(x, y)=\frac{1}{2 \pi \sigma_{1} \sigma_{2} \sqrt{1-\rho^{2}}} \exp \left\{-\frac{1}{2} Q(x, y)\right\} \quad-\infty<x, y<\infty
$$

where

$$
Q(x, y)=\frac{1}{1-\rho^{2}}\left[\left(\frac{x-\mu_{1}}{\sigma_{1}}\right)^{2}-2 \rho \frac{\left(x-\mu_{1}\right)}{\sigma_{1}} \frac{\left(y-\mu_{2}\right)}{\sigma_{2}}+\left(\frac{y-\mu_{1}}{\sigma_{2}}\right)^{2}\right]
$$

It can be shown that

$$
E(X)=\mu_{1}, E(Y)=\mu_{2}, \operatorname{Var}(X)=\sigma_{1}^{2}, \operatorname{Var}(Y)=\sigma_{2}^{2}
$$


and $\rho$ is the correlation between $X$ and $Y$, where $-\infty<\mu_{1}, \mu_{2}<\infty, \sigma_{1}^{2}, \sigma_{2}^{2}>0$ and $-1 \leq \rho \leq 1$

Suppose that $n$ observations are taken from this Bivariate Normal Distribution, let say $\left(x_{1}, y_{1}\right),\left(x_{2}, y_{2}\right), \ldots,\left(x_{n}, y_{n}\right)$. The Maximum Likelihood Estimators, MLEs, of the parameters $\mu_{1}, \mu_{2}, \sigma_{1}, \sigma_{2}$ and $\rho$ are:

$$
\begin{array}{r}
\hat{\mu}_{1}=\frac{\sum_{i=1}^{n} x_{i}}{n}=\bar{x} \\
\hat{\mu}_{2}=\frac{\sum_{i=1}^{n} y_{i}}{n}=\bar{y} \\
\hat{\sigma}_{1}^{2}=\frac{\sum_{i=1}^{n}\left(x_{i}-\bar{x}\right)^{2}}{n} \\
\hat{\sigma}_{2}^{2}=\frac{\sum_{i=1}^{n}\left(y_{i}-\bar{y}\right)^{2}}{n} \\
r_{n}=\frac{\sum_{i=1}^{n}\left(x_{i}-\bar{x}\right)\left(y_{i}-\bar{y}\right)}{\sqrt{\sum_{i=1}^{n}\left(x_{i}-\bar{x}\right) \sum_{i=1}^{n}\left(y_{i}-\bar{y}\right)}}
\end{array}
$$

It can be shown that the properties of the estimator of the correlation coefficiente $r_{n}$ for the general case of the Bivariate Normal distribution are the following:

\section{Properties of $r_{n}$}

1. $\sqrt{n}\left(r_{n}-\rho\right) \rightarrow N\left(0,\left(1-\rho^{2}\right)^{2}\right)$

2. $\sqrt{n}\left(\frac{1}{2} \ln \left(\frac{1+r_{n}}{1-r_{n}}\right)-\frac{1}{2} \ln \left(\frac{1+\rho}{1-\rho}\right)\right) \rightarrow N(0,1)$. This is called Fisher's $z$-Transformation 


\subsection{Case of Equal Variances}

\subsubsection{Data and Model}

Suppose $(X, Y)$ is a random vector following a Bivariate Normal distribution $N_{2}(\boldsymbol{\mu}, \Sigma)$ where

$$
\boldsymbol{\mu}=\left(\mu_{1}, \mu_{2}\right)^{\prime}
$$

and $\Sigma$ is the $2 \times 2$ variance-covariance matrix. In the present section we assume that $\operatorname{Var}(X)=\operatorname{Var}(Y)=\sigma^{2}$ and $\operatorname{Cov}(X, Y)=\sigma^{2} \rho$. Then, the joint probability density function for $(X, Y)$ is given by

$$
f\left(x, y ; \mathbf{u}, \sigma^{2}, \rho\right)=\frac{1}{2 \pi \sigma^{2} \sqrt{1-\rho^{2}}} \exp \left\{-\frac{W(x, y)}{2 \sigma^{2}\left(1-\rho^{2}\right)}\right\}
$$

where

$$
W(x, y)=\left(x-\mu_{1}\right)^{2}-2 \rho\left(x-\mu_{1}\right)\left(y-\mu_{2}\right)+\left(y-\mu_{1}\right)^{2}
$$

provided that $-1<\rho<1$.

\subsubsection{Likelihood Function}

We want to find the MLEs of the unkown parameters of the distribution $\mu_{1}, \mu_{2}, \tau$ and $\rho$ where $\tau=\sigma^{2}$. For this purpose, let us assume $\left(x_{1}, y_{1}\right),\left(x_{2}, y_{2}\right), \ldots,\left(x_{n}, y_{n}\right)$ is a random sample from this population. Since they are independents and identically distributed, we can write the likelihood function $L\left(\mathbf{u}, \sigma^{2}, \rho ;(\boldsymbol{x}, \boldsymbol{y})\right)$ as

$$
\begin{aligned}
L\left(\mathbf{u}, \sigma^{2}, \rho ;(\boldsymbol{x}, \boldsymbol{y})\right) & =\prod_{i=1}^{n} f\left(x_{i}, y_{i} ; \mathbf{u}, \sigma^{2}, \rho\right) \\
& =\prod_{i=1}^{n} \frac{1}{2 \pi \tau \sqrt{1-\rho^{2}}} \exp \left\{-\frac{1}{2 \tau\left(1-\rho^{2}\right)} Q\left(x_{i}, y_{i}\right)\right\}
\end{aligned}
$$


where $\boldsymbol{x}=\left(x_{1}, x_{2}, \ldots, x_{n}\right), \boldsymbol{y}=\left(y_{1}, y_{2}, \ldots, y_{n}\right)$ and

$$
Q\left(x_{i}, y_{i}\right)=\left(x_{i}-\mu_{1}\right)^{2}-2 \rho\left(x_{i}-\mu_{1}\right)\left(y_{i}-\mu_{2}\right)+\left(y_{i}-\mu_{2}\right)^{2} .
$$

The likelihood function can be written as

$$
L\left(\mathbf{u}, \sigma^{2}, \rho ;(\boldsymbol{x}, \boldsymbol{y})\right)=\frac{1}{(2 \pi)^{n} \tau^{n}\left(1-\rho^{2}\right)^{\frac{n}{2}}} \exp \left\{-\frac{1}{2 \tau\left(1-\rho^{2}\right)} Q^{*}(x, y)\right\}
$$

where

$$
Q^{*}(x, y)=\sum_{i=1}^{n}\left[\left(x_{i}-\mu_{1}\right)^{2}-2 \rho\left(x_{i}-\mu_{1}\right)\left(y_{i}-\mu_{2}\right)+\left(y_{i}-\mu_{2}\right)^{2}\right] .
$$

\subsubsection{Derivation of MLEs of Covariance Matrix Symmetric}

\section{Bivariate Normal Parameters}

Using the likelihood function $L\left(\mathbf{u}, \sigma^{2}, \rho ;(\boldsymbol{x}, \boldsymbol{y})\right)$ we want to find the MLEs of $\hat{\mu_{1}}, \hat{\mu_{2}}, \hat{\sigma_{1}}, \hat{\sigma_{2}}, \hat{\rho}$ that maximizes $L\left(\mathbf{u}, \sigma^{2}, \rho ;(\boldsymbol{x}, \boldsymbol{y})\right)$.

For this purpose, it is convenient to work with the natural logarithm of the likelihood function (since it is a monotone function)

$$
\begin{aligned}
\ln L\left(\mathbf{u}, \sigma^{2}, \rho ;(\boldsymbol{x}, \boldsymbol{y})\right)= & -n \ln (2 \pi)-n \ln \tau-\frac{n}{2} \ln \left(1-\rho^{2}\right) \\
& -\frac{1}{2 \tau\left(1-\rho^{2}\right)}\left[\sum_{i=1}^{n}\left[\left(x_{i}-\mu_{1}\right)^{2}-2 \rho\left(x_{i}-\mu_{1}\right)\left(y_{i}-\mu_{2}\right)+\left(y_{i}-\mu_{2}\right)^{2}\right]\right]
\end{aligned}
$$

and solving the system of equations

$$
\frac{\partial \ln L}{\partial \mu_{1}}=\frac{\partial \ln L}{\partial \mu_{2}}=\frac{\partial \ln L}{\partial \tau}=\frac{\partial \ln L}{\partial \rho}=0
$$


we can obtain the desired estimators.

The equations in (2.16 can be written as

$$
\begin{aligned}
& \frac{\partial \ln L}{\partial \mu_{1}}=-\frac{1}{2 \tau\left(1-\rho^{2}\right)} \sum_{i=1}^{n}\left[-2\left(x_{i}-\mu_{1}\right)+2 \rho\left(y_{i}-\mu_{2}\right)\right]=0 \\
& \frac{\partial \ln L}{\partial \mu_{2}}=-\frac{1}{2 \tau\left(1-\rho^{2}\right)} \sum_{i=1}^{n}\left[2 \rho\left(x_{i}-\mu_{1}\right)-2\left(y_{i}-\mu_{2}\right)\right]=0 \\
& \frac{\partial \ln L}{\partial \tau}=-\frac{n}{\tau}+\frac{\sum_{i=1}^{n}\left[\left(x_{i}-\mu_{1}\right)^{2}+\left(y_{i}-\mu_{2}\right)^{2}-2 \rho\left(x_{i}-\mu_{1}\right)\left(y_{i}-\mu_{2}\right)\right]}{2\left(1-\rho^{2}\right) \tau^{2}}=0 \\
& \frac{\partial \ln L}{\partial \rho}=\frac{2 n \rho}{2\left(1-\rho^{2}\right)}-\frac{\left[-2 \sum_{i=1}^{n}\left(x_{i}-\mu_{1}\right)\left(y_{i} \mu_{2}\right)\right]\left[2 \tau\left(1-\rho^{2}\right)\right]}{4 \tau^{2}\left(a-\rho^{2}\right)^{2}} \\
&-\frac{2 \tau(-2 \rho) \sum_{i=1}^{n}\left[\left(x_{i}-\mu_{1}\right)^{2}+\left(y_{i}-\mu_{2}\right)^{2}-2 \rho\left(x_{i}-\mu_{1}\right)\left(y_{i}-\mu_{2}\right)\right]}{4 \tau^{2}\left(a-\rho^{2}\right)^{2}}=0
\end{aligned}
$$

Simplifying equations (2.17) and (2.18), we have

$$
\begin{array}{r}
-2 \sum_{i=1}^{n}\left(x_{i}-\mu_{1}\right)+2 \rho \sum_{i=1}^{n}\left(y_{i}-\mu_{2}\right)=0 \\
2 \rho \sum_{i=1}^{n}\left(x_{i}-\mu_{1}\right)-2 \sum_{i=1}^{n}\left(y_{i}-\mu_{2}\right)=0
\end{array}
$$

multiplying equation (2.21) by $\rho$ and adding it to equation (2.22), we obtain

$$
2\left(\rho^{2}-1\right) \sum_{i=1}^{2}\left(y_{1}-\mu_{2}\right)=0
$$

from where

$$
\sum_{i=1}^{n}\left(y_{i}-\mu_{2}\right)=0
$$

since $\rho \neq \pm 1$, which implies that

$$
\hat{\mu}_{2}=\frac{\sum_{i=1}^{n} y_{i}}{n}=\bar{y}
$$

In similar way, we obtain

$$
\hat{\mu}_{1}=\frac{\sum_{i=1}^{n} x_{i}}{n}=\bar{x}
$$


Now, in order to obtain $\hat{\rho}$ and $\hat{\tau}$, let us use equations (2.19) and (2.20). Finding the common denominator in (2.19) and replacing $\mu_{1}$ by $\bar{x}$ and $\mu_{2}$ by $\bar{y}$, we get

$$
\frac{-2 n \tau\left(1-\rho^{2}\right)+\sum_{i=1}^{n}\left[\left(x_{i}-\bar{x}\right)^{2}+\left(y_{i}-\bar{y}\right)^{2}\right]-2 \rho \sum_{i=1}^{n}\left(x_{i}-\bar{x}\right)\left(y_{i}-\bar{y}\right)}{2 \tau^{2}\left(1-\rho^{2}\right)}=0
$$

and multiplying it by $2 \tau^{2}\left(1-\rho^{2}\right)$ we can obtain

$$
-2 n \tau\left(1-\rho^{2}\right)+\sum_{i=1}^{n}\left[\left(x_{i}-\bar{x}\right)^{2}+\left(y_{i}-\bar{y}\right)^{2}\right]-2 \rho \sum_{i=1}^{n}\left(x_{i}-\bar{x}\right)\left(y_{i}-\bar{y}\right)=0
$$

Similarly, simplifying equation (2.20) and replacing $\mu_{1}$ and $\mu_{2}$ by their estimators we have

$$
\begin{aligned}
\frac{n \rho}{\left(1-\rho^{2}\right)}+\frac{\sum_{i=1}^{n}\left(x_{i}-\bar{x}\right)\left(y_{i}-\bar{y}\right)}{\tau\left(1-\rho^{2}\right)}- & \frac{\rho \sum_{i=1}^{n}\left[\left(x_{i}-\bar{x}\right)^{2}+\left(y_{i}-\bar{y}\right)^{2}\right]}{\tau\left(1-\rho^{2}\right)^{2}} \\
& +\frac{2 \rho^{2} \sum_{i=1}^{n}\left(x_{i}-\bar{x}\right)\left(y_{i}-\bar{y}\right)}{\tau\left(1-\rho^{2}\right)^{2}}=0
\end{aligned}
$$

and multiplying it by $\tau\left(1-\rho^{2}\right)^{2}$ and combining like terms, equation (2.29) becomes

$$
n \tau \rho\left(1-\rho^{2}\right)+\left(1-\rho^{2}+2 \rho^{2}\right) \sum_{i=1}^{n}\left(x_{i}-\bar{x}\right)\left(y_{i}-\bar{y}\right)-\rho \sum_{i=1}^{n}\left[\left(x_{i}-\bar{x}\right)^{2}+\left(y_{i}-\bar{y}\right)^{2}\right]=0
$$

which is the same as

$$
n \tau \rho\left(1-\rho^{2}\right)-\rho \sum_{i=1}^{n}\left[\left(x_{i}-\bar{x}\right)^{2}+\left(y_{i}-\bar{y}\right)^{2}\right]+\left(1+\rho^{2}\right) \sum_{i=1}^{n}\left(x_{i}-\bar{x}\right)\left(y_{i}-\bar{y}\right)=0
$$

Multiplying equation (2.28) by $\rho$ and equation (2.31) by 2 and adding them up, we get

$$
-\rho \sum_{i=1}^{n}\left[\left(x_{i}-\bar{x}\right)^{2}+\left(y_{i}-\bar{y}\right)^{2}\right]+2 \sum_{i=1}^{n}\left(x_{i}-\bar{x}\right)\left(y_{i}-\bar{y}\right)=0
$$


from where

$$
\hat{\rho}=\frac{2 \sum_{i=1}^{n}\left(x_{i}-\bar{x}\right)\left(y_{i}-\bar{y}\right)}{\sum_{i=1}^{n}\left[\left(x_{i}-\bar{x}\right)^{2}+\left(y_{i}-\bar{y}\right)^{2}\right]}
$$

which is different from $\hat{r}_{n}$ obtained in (2.7).

Finally, solving for $\tau$ we obtain that

$$
\hat{\tau}=\frac{\sum_{i=1}^{n}\left[\left(x_{i}-\bar{x}\right)^{2}+\left(y_{i}-\bar{y}\right)^{2}-2 \hat{\rho}\left(x_{i}-\bar{x}\right)\left(y_{i}-\bar{y}\right)\right]}{2 n\left(1-\hat{\rho}^{2}\right)}
$$

which is equivalent to

$$
\hat{\tau}=\frac{\sum_{i=1}^{n}\left[\left(x_{i}-\bar{x}\right)^{2}+\left(y_{i}-\bar{y}\right)^{2}\right]}{2 n}
$$

and thus

$$
\hat{\sigma}=\sqrt{\frac{\sum_{i=1}^{n}\left[\left(x_{i}-\bar{x}\right)^{2}+\left(y_{i}-\bar{y}\right)^{2}\right]}{2 n}}
$$

\subsubsection{Properties of the MLEs of the Covariance Matrix Sym-}

\section{metric Bivariate Normal Parameters}

1. Mean of $\hat{\mu}_{1}, \hat{\mu}_{2}$ :

The estimators of $\hat{\mu}_{1}$ and $\hat{\mu}_{2}$ in our model are unbiased estimators. It can be easily proven:

Proof.

$$
\begin{aligned}
E\left[\hat{\mu}_{1}\right] & =E\left[\frac{\sum_{i=1}^{n} X_{i}}{n}\right] \\
& =\frac{1}{n} E\left[\sum_{i=1}^{n} X_{i}\right] \\
& =\frac{1}{n} \sum_{i=1}^{n} E\left[X_{i}\right]
\end{aligned}
$$


and using the fact that $E\left[X_{i}\right]=\mu_{1}$ then

$$
\begin{aligned}
E\left[\hat{\mu}_{1}\right] & =\frac{1}{n} \sum_{i=1}^{n} \mu_{1} \\
& =\frac{1}{n} n \mu_{1} \\
& =\mu_{1}
\end{aligned}
$$

Therefore $E\left[\hat{\mu}_{1}\right]=\mu_{1}$ which means that $\hat{\mu}_{1}$ is an unbiased estimator of $\mu_{1}$

Similarly, $\hat{\mu}_{2}$ is an unbiased estimator of $\mu_{2}$.

2. Mean of $\hat{\mu}_{1}-\hat{\mu}_{2}$ :

It is easy to show that the mean of $\hat{\mu}_{1}-\hat{\mu}_{2}$ is just $\mu_{1}-\mu_{2}$.

3. Variance of $\hat{\mu}_{1}, \hat{\mu}_{2}$ :

The variance of $\hat{\mu}_{1}$ and $\hat{\mu}_{1}$ is $\frac{\sigma^{2}}{n}$

Proof.

$$
\begin{aligned}
\operatorname{Var}\left(\hat{\mu}_{1}\right) & =\operatorname{Var}\left(\frac{\sum_{i=1}^{n} X_{i}}{n}\right) \\
& =\frac{1}{n^{2}} \operatorname{Var}\left(\sum_{i=1}^{n} X_{i}\right)
\end{aligned}
$$

Since $X_{i}, i=1, \ldots, n$ are independent, then

$$
\begin{aligned}
\operatorname{Var}\left(\hat{\mu}_{1}\right) & =\frac{1}{n^{2}} \sum_{i=1}^{n} \operatorname{Var}\left(X_{i}\right) \\
& =\frac{1}{n^{2}} \sum_{i=1}^{n} \sigma^{2} \\
& =\frac{1}{n^{2}} n \sigma^{2} \\
& =\frac{\sigma^{2}}{n}
\end{aligned}
$$

Therefore $\operatorname{Var}\left(\hat{\mu}_{1}\right)=\frac{\sigma^{2}}{n}$ 
4. Variance of $\hat{\mu}_{1}-\hat{\mu}_{2}$ :

The variance of $\hat{\mu}_{1}-\hat{\mu}_{2}$ is given by $\frac{2 \sigma^{2}}{n}(1-\rho)$

Proof.

$$
\begin{aligned}
\operatorname{Var}\left(\hat{\mu}_{1}-\hat{\mu}_{2}\right) & =\operatorname{Var}\left(\hat{\mu}_{1}\right)+\operatorname{Var}\left(\hat{\mu}_{2}\right)-2 \operatorname{Cov}\left(\hat{\mu}_{1}, \hat{\mu}_{2}\right) \\
& =\frac{\sigma^{2}}{n}+\frac{\sigma^{2}}{n}-2 \operatorname{Cov}\left(\frac{\sum_{i=1}^{n} X_{i}}{n}, \frac{\sum_{j=1}^{n} Y_{j}}{n}\right) \\
& =\frac{2 \sigma^{2}}{n}-\frac{2}{n^{2}} \sum_{i=1}^{n} \sum_{j=1}^{n} \operatorname{Cov}\left(X_{i}, Y_{j}\right)
\end{aligned}
$$

since $\operatorname{Cov}\left(X_{i}, Y_{j}\right)=\sigma^{2} \rho$ if $i=j$ and $\operatorname{Cov}\left(X_{i}, Y_{j}\right)=0$ if $i \neq j$, then

$$
\begin{aligned}
\operatorname{Var}\left(\hat{\mu}_{1}-\hat{\mu}_{2}\right) & =\frac{2 \sigma^{2}}{n}-\frac{2}{n^{2}} \sum_{i=1}^{n} \sigma^{2} \rho \\
& =\frac{2 \sigma^{2}}{n}-\frac{2}{n^{2}} \frac{\sigma^{2} \rho}{n} \\
& =\frac{2 \sigma^{2}}{n}-\frac{2 \sigma^{2} \rho}{n} \rho \\
& =\frac{2 \sigma^{2}}{n}(1-\rho)
\end{aligned}
$$

\subsubsection{Sampling Distributions of MLEs of the Covariance}

\section{Matrix Symmetric Bivariate Normal Parameters}

1. The distribution of $\hat{\mu}_{1}-\hat{\mu}_{2}$

Since $\hat{\mu}_{1}-\hat{\mu}_{2}$ is a linear combinations of $\left(X_{i}, Y_{i}\right), i=1, \ldots, n$ and those come from a Bivariate Normal populations, the linear combination is also a normal population. From the properties seen in Section 2.2.4, then we can conclude that $\hat{\mu}_{1}-\hat{\mu}_{2} \sim N\left(\mu_{1}-\mu_{2}, \frac{2 \sigma^{2}}{n}(1-\rho)\right)$. 


\subsubsection{Discussion about the correlation coefficient $\hat{\rho}$}

In subsection we will discuss some properties and the limiting distribution of the estimator $r_{n}$ of the correlation coefficient $\rho$ that was found in Section 2.2.3.

Recall that for the general case of the Bivariate Normal Distribution, the estimator $\hat{r}_{n}$ for the correlation coefficient $\rho$ is given by

$$
\hat{r}_{n}=\frac{\sum_{i=1}^{n}\left(x_{i}-\bar{x}\right)\left(y_{i}-\bar{y}\right)}{\sqrt{\left(\sum_{i=1}^{n}\left(x_{i}-\bar{x}\right)^{2}\right)\left(\sum_{i=1}^{n}\left(y_{i}-\bar{y}\right)^{2}\right)}}
$$

while the estimator for the correlation coefficient in case of equal variances $\hat{\rho}$ was found to be

$$
\hat{\rho}_{n}=\frac{2 \sum_{i=1}^{n}\left(x_{i}-\bar{x}\right)\left(y_{i}-\bar{y}\right)}{\sum_{i=1}^{n}\left[\left(x_{i}-\bar{x}\right)^{2}+\left(y_{i}-\bar{y}\right)^{2}\right]}
$$

\section{Properties of $\hat{\rho}_{n}$}

1. $-1 \leq \hat{\rho}_{n} \leq 1$

Proof.

$$
\begin{aligned}
\hat{\rho}_{n} \leq 1 & \Leftrightarrow \frac{2 \sum_{i=1}^{n}\left(x_{i}-\bar{x}\right)\left(y_{i}-\bar{y}\right)}{\sum_{i=1}^{n}\left[\left(x_{i}-\bar{x}\right)^{2}+\left(y_{i}-\bar{y}\right)^{2}\right]} \leq 1 \\
& \Leftrightarrow 2 \sum_{i=1}^{n}\left(x_{i}-\bar{x}\right)\left(y_{i}-\bar{y}\right) \leq \sum_{i=1}^{n}\left[\left(x_{i}-\bar{x}\right)^{2}+\left(y_{i}-\bar{y}\right)^{2}\right] \\
& \Leftrightarrow \sum_{i=1}^{n}\left[\left(x_{i}-\bar{x}\right)^{2}+\left(y_{i}-\bar{y}\right)^{2}\right]-2 \sum_{i=1}^{n}\left(x_{i}-\bar{x}\right)\left(y_{i}-\bar{y}\right) \geq 0 \\
& \Leftrightarrow \sum_{i=1}^{n}\left[\left(x_{i}-\bar{x}\right)^{2}+\left(y_{i}-\bar{y}\right)^{2}-2\left(x_{i}-\bar{x}\right)\left(y_{i}-\bar{y}\right)\right] \geq 0 \\
& \Leftrightarrow \sum_{i=1}^{n}\left[\left(x_{i}-\bar{x}\right)+\left(y_{i}-\bar{y}\right)\right]^{2} \geq 0
\end{aligned}
$$

In similar way, $\hat{\rho}_{n} \geq-1$.

2. $P\left(-1 \leq \hat{\rho}_{n} \leq 1\right)=1$ 
Proof.

$$
\begin{aligned}
P\left(\hat{\rho}_{n}=1\right) & =P\left(\sum_{i=1}^{n}\left[\left(X_{i}-\bar{X}\right)^{2}+\left(Y_{i}-\bar{Y}\right)^{2}\right]=0\right) \\
= & P\left(\bigcap_{i=1}^{n}\left[\left(X_{i}-\bar{X}\right)^{2}+\left(Y_{i}-\bar{Y}\right)^{2}\right]=0\right) \\
& \leq P\left(\left(X_{i}-\bar{X}\right)^{2}+\left(Y_{i}-\bar{Y}\right)^{2}=0\right)=0
\end{aligned}
$$

3. $\hat{\rho}_{n}$ and $\hat{r}_{n}$ have the same sign and $\left|\hat{\rho}_{n}\right| \leq\left|r_{n}\right|$

Proof. The desired inequality is equivalent to

$$
\begin{aligned}
& \frac{2\left|\sum_{i=1}^{n}\left(x_{i}-\bar{x}\right)\left(y_{i}-\bar{y}\right)\right|}{\sum_{i=1}^{n}\left(x_{i}-\bar{x}\right)^{2}+\sum_{i=1}^{n}\left(y_{i}-\bar{y}\right)^{2}} \leq \frac{\left|\sum_{i=1}^{n}\left(x_{i}-\bar{x}\right)\left(y_{i}-\bar{y}\right)\right|}{\sqrt{\sum_{i=1}^{n}\left(x_{i}-\bar{x}\right)^{2} \sum_{i=1}^{n}\left(y_{i}-\bar{y}\right)^{2}}} \\
& \Leftrightarrow 4\left(\sum_{i=1}^{n}\left(x_{i}-\bar{x}\right)^{2}\right)\left(\sum_{i=1}^{n}\left(y_{i}-\bar{y}\right)^{2}\right) \leq\left[\sum_{i=1}^{n}\left(x_{i}-\bar{x}\right)^{2}+\sum_{i=1}^{n}\left(y_{i}-\bar{y}\right)^{2}\right]^{2} \\
& \Leftrightarrow\left(\sum_{i=1}^{n}\left(x_{i}-\bar{x}\right)^{2}\right)^{2}+\left(\sum_{i=1}^{n}\left(y_{i}-\bar{y}\right)^{2}\right)^{2}-2\left(\sum_{i=1}^{n}\left(x_{i}-\bar{x}\right)^{2}\right)\left(\sum_{i=1}^{n}\left(y_{i}-\bar{y}\right)^{2}\right) \geq 0
\end{aligned}
$$

which is certainly true. Therefore, $\left|\hat{\rho}_{n}\right| \leq\left|r_{n}\right|$

\section{The limiting distribution of $\hat{\rho_{n}}$}

In the present subsection we will show that as the sample size goes to infinity $(n \rightarrow \infty)$ it holds that $\sqrt{n}\left(\hat{\rho}_{n}-\rho\right) \rightarrow N\left(0, \gamma^{2}\right)$ in distribution where the constant $\gamma^{2}$ will be determined later.

To show this asymptotic normality the following results are needed.

Result 2.2.1. $\sqrt{n}\left(\bar{X}_{n}-\mu_{1}\right)^{2} \rightarrow 0$ in probability as $n \rightarrow \infty$ 
Proof. For any fixed $\epsilon>0$, by Chebychev's inequality, it holds that

$$
\begin{aligned}
P\left(\left|\sqrt{n}\left(\bar{X}_{n}-\mu_{1}\right)^{2}\right|>\epsilon\right) & \leq \frac{E\left[\sqrt{n}\left(\bar{X}_{n}-\mu_{1}\right)^{2}\right]}{\epsilon} \\
& =\frac{\sqrt{n} \operatorname{Var}\left(\bar{X}_{n}\right)}{n \epsilon} \\
& =\frac{\sqrt{n} \sigma^{2}}{n \epsilon} \\
& =\frac{\sigma^{2}}{\sqrt{n} \epsilon} \rightarrow 0
\end{aligned}
$$

as $n \rightarrow \infty$

Result 2.2.2. $\sqrt{n}\left(\bar{Y}_{n}-\mu_{2}\right)^{2} \rightarrow 0$ in probability as $n \rightarrow \infty$

It can be shown in the similar way as in 1.

Result 2.2.3. $\sqrt{n}\left(\bar{X}_{n}-\mu_{1}\right)\left(\bar{Y}_{n}-\mu_{2}\right) \rightarrow 0$ in probability as $n \rightarrow \infty$

Proof. For any given $\epsilon>0$ we have

$$
\begin{aligned}
P\left(\left|\sqrt{n}\left(\bar{X}_{n}-\mu_{1}\right)\left(\bar{Y}_{n}-\mu_{2}\right)\right|>\epsilon\right) & \leq \frac{\sqrt{n}}{\epsilon} E\left|\left(\bar{X}_{n}-\mu_{1}\right)\left(\bar{Y}_{n}-\mu_{2}\right)\right| \\
& \leq \frac{\sqrt{n}}{\epsilon} \sqrt{E\left(\bar{X}_{n}-\mu_{1}\right)^{2} E\left(\bar{Y}_{n}-\mu_{2}\right)^{2}} \\
& =\frac{\sqrt{n}}{\epsilon} \frac{\sigma^{2}}{n} \\
& =\frac{\sigma^{2}}{\epsilon \sqrt{n}} \rightarrow 0
\end{aligned}
$$

as $n \rightarrow \infty$, where the first inequality follows from Chebychev's inequality and the second one is from Cauchy-Schwarz inequality. 
Lemma 2.2.4. As $n \rightarrow \infty$ the limit distribution of

$$
\begin{array}{r}
\left(\sqrt{n}\left[\frac{1}{n} \sum_{i=1}^{n}\left(X_{i}-\bar{X}\right)\left(Y_{i}-\bar{Y}\right)-\operatorname{Cov}(X, Y)\right],\right. \\
\sqrt{n}\left[\frac{1}{n} \sum_{i=1}^{n}\left(X_{i}-\bar{X}\right)^{2}-\sigma^{2}\right], \\
\left.\sqrt{n}\left[\frac{1}{n} \sum_{i=1}^{n}\left(Y_{i}-\bar{Y}\right)^{2}-\sigma^{2}\right]\right)
\end{array}
$$

is the same as

$$
\begin{array}{r}
\left(\sqrt{n}\left[\frac{1}{n} \sum_{i=1}^{n}\left(X_{i}-\mu_{1}\right)\left(Y_{i}-\mu_{2}\right)-\operatorname{Cov}(X, Y)\right],\right. \\
\sqrt{n}\left[\frac{1}{n} \sum_{i=1}^{n}\left(X_{i}-\mu_{1}\right)^{2}-\sigma^{2}\right], \\
\left.\sqrt{n}\left[\frac{1}{n} \sum_{i=1}^{n}\left(Y_{i}-\mu_{2}\right)^{2}-\sigma^{2}\right]\right)
\end{array}
$$

Proof. It is easy to see that

$$
\begin{aligned}
\sum_{i=1}^{n} & \left(X_{i}-\bar{X}\right)\left(Y_{i}-\bar{Y}\right) \\
= & \sum_{i=1}^{n}\left[\left(X_{i}-\mu_{1}\right)-\left(\bar{X}-\mu_{1}\right)\right]\left[\left(Y_{i}-\mu_{2}\right)-\left(\bar{Y}-\mu_{2}\right)\right] \\
= & \sum_{i=1}^{n}\left[\left(X_{i}-\mu_{1}\right)\left(Y_{i}-\mu_{2}\right)-\left(X_{i}-\mu_{1}\right)\left(\bar{Y}-\mu_{2}\right)\right. \\
& \left.-\left(\bar{X}-\mu_{1}\right)\left(Y_{i}-\mu_{2}\right)+\left(\bar{X}-\mu_{1}\right)\left(\bar{Y}-\mu_{2}\right)\right] \\
= & \sum_{i=1}^{n}\left(X_{i}-\mu_{1}\right)\left(Y_{i}-\mu_{2}\right)-n\left(\bar{X}-\mu_{1}\right)\left(\bar{Y}-\mu_{2}\right) .
\end{aligned}
$$

It yields

$$
\begin{aligned}
& \sqrt{n}\left[\frac{1}{n} \sum_{i=1}^{n}\left(X_{i}-\bar{X}\right)\left(Y_{i}-\bar{Y}\right)-\operatorname{Cov}(X, Y)\right] \\
& =\sqrt{n}\left[\frac{1}{n} \sum_{i=1}^{n}\left(X_{i}-\mu_{1}\right)\left(Y_{i}-\mu_{2}\right)-\operatorname{Cov}(X, Y)-\left(\bar{X}-\mu_{1}\right)\left(\bar{Y}-\mu_{2}\right)\right] \\
& =\sqrt{n}\left[\frac{1}{n} \sum_{i=1}^{n}\left(X_{i}-\mu_{1}\right)\left(Y_{i}-\mu_{2}\right)-\operatorname{Cov}(X, Y)\right]-\sqrt{n}\left(\bar{X}-\mu_{1}\right)\left(\bar{Y}-\mu_{2}\right)
\end{aligned}
$$


In the same manner we can obtain

$$
\sqrt{n}\left[\frac{1}{n} \sum_{i=1}^{n}\left(X_{i}-\bar{X}\right)^{2}-\sigma^{2}\right]=\sqrt{n}\left[\frac{1}{n} \sum_{i=1}^{n}\left(X_{i}-\mu_{1}\right)^{2}-\sigma^{2}\right]-\sqrt{n}\left(\bar{X}-\mu_{1}\right)^{2}
$$

and

$$
\sqrt{n}\left[\frac{1}{n} \sum_{i=1}^{n}\left(Y_{i}-\bar{Y}\right)^{2}-\sigma^{2}\right]=\sqrt{n}\left[\frac{1}{n} \sum_{i=1}^{n}\left(Y_{i}-\mu_{2}\right)^{2}-\sigma^{2}\right]-\sqrt{n}\left(\bar{Y}-\mu_{2}\right)^{2}
$$

Using the Results 2.2.1 - 2.2.3 obtained above, we can conclude that the desired result is true.

Lemma 2.2.5. The limiting distribution of

$$
\sqrt{n}\left(\hat{\rho}_{n}-\rho\right)
$$

is the same as

$$
\sqrt{n}\left(\widetilde{\rho}_{n}-\rho\right)
$$

where

$$
\widetilde{\rho}_{n}=\frac{2 \sum_{i=1}^{n}\left(X_{1}-\mu_{1}\right)\left(Y_{1}-\mu_{2}\right)}{\sum_{i=1}^{n}\left(X_{i}-\mu_{1}\right)^{2}+\sum_{i=1}^{n}\left(Y_{i}-\mu_{2}\right)^{2}}
$$

Proof. Obviously

$$
\begin{aligned}
& \frac{\sqrt{n}}{2}\left(\hat{\rho}_{n}-\widetilde{\rho}_{n}\right) \\
& =\frac{1}{2} \sqrt{n}\left(\frac{2 \sum_{i=1}^{n}\left(X_{i}-\bar{X}\right)\left(Y_{i}-\bar{Y}\right)}{\sum_{i=1}^{n}\left(X_{i}-\bar{X}\right)^{2}+\sum_{i=1}^{n}\left(Y_{i}-\bar{Y}\right)^{2}}-\frac{2 \sum_{i=1}^{n}\left(x_{1}-\mu_{1}\right)\left(y_{1}-\mu_{2}\right)}{\sum_{i=1}^{n}\left(x_{i}-\mu_{1}\right)^{2}+\sum_{i=1}^{n}\left(y_{i}-\mu_{2}\right)^{2}}\right) \\
& =\frac{\sqrt{n} \sum_{i=1}^{n}\left(X_{i}-\bar{X}\right)\left(Y_{i}-\bar{Y}\right)}{\sum_{i=1}^{n}\left(X_{i}-\bar{X}\right)^{2}+\sum_{i=1}^{n}\left(Y_{i}-\bar{Y}\right)^{2}}-\frac{\sqrt{n} \sum_{i=1}^{n}\left(X_{i}-\mu_{1}\right)\left(Y_{i}-\mu_{2}\right)}{\sum_{i=1}^{n}\left(X_{i}-\mu_{1}\right)^{2}+\sum_{i=1}^{n}\left(Y_{i}-\mu_{2}\right)^{2}}
\end{aligned}
$$

adding and subtracting

$$
\sqrt{n} \frac{\sum_{i=1}^{n}\left(X_{i}-\mu_{1}\right)\left(Y_{i}-\mu_{2}\right)}{\sum_{i=1}^{n}\left(X_{i}-\bar{X}\right)^{2}+\sum_{i=1}^{n}\left(Y_{i}-\bar{Y}\right)^{2}}
$$


then we get

$$
\begin{aligned}
& \frac{\sqrt{n}}{2}\left(\hat{\rho}_{n}-\widetilde{\rho}_{n}\right) \\
& =\frac{\sqrt{n} \sum_{i=1}^{n}\left(X_{i}-\bar{X}\right)\left(Y_{i}-\bar{Y}\right)}{\sum_{i=1}^{n}\left(X_{i}-\bar{X}\right)^{2}+\sum_{i=1}^{n}\left(Y_{i}-\bar{Y}\right)^{2}}-\frac{\sqrt{n} \sum_{i=1}^{n}\left(X_{i}-\mu_{1}\right)\left(Y_{i}-\mu_{2}\right)}{\sum_{i=1}^{n}\left(X_{i}-\mu_{1}\right)^{2}+\sum_{i=1}^{n}\left(Y_{i}-\mu_{2}\right)^{2}} \\
& \quad \sqrt{n} \frac{\sum_{i=1}^{n}\left(X_{i}-\mu_{1}\right)\left(Y_{i}-\mu_{2}\right)}{\sum_{i=1}^{n}\left(X_{i}-\bar{X}\right)^{2}+\sum_{i=1}^{n}\left(Y_{i}-\bar{Y}\right)^{2}}-\sqrt{n} \frac{\sum_{i=1}^{n}\left(X_{i}-\mu_{1}\right)\left(Y_{i}-\mu_{2}\right)}{\sum_{i=1}^{n}\left(X_{i}-\bar{X}\right)^{2}+\sum_{i=1}^{n}\left(Y_{i}-\bar{Y}\right)^{2}}
\end{aligned}
$$

grouping them

$$
\begin{aligned}
& \frac{\sqrt{n}}{2}\left(\hat{\rho}_{n}-\widetilde{\rho}_{n}\right) \\
& =\left[g \frac{\sqrt{n} \sum_{i=1}^{n}\left(X_{i}-\bar{X}\right)\left(Y_{i}-\bar{Y}\right)}{\sum_{i=1}^{n}\left(X_{i}-\bar{X}\right)^{2}+\sum_{i=1}^{n}\left(Y_{i}-\bar{Y}\right)^{2}}-\frac{\sqrt{n} \sum_{i=1}^{n}\left(X_{i}-\mu_{1}\right)\left(Y_{i}-\mu_{2}\right)}{\sum_{i=1}^{n}\left(X_{i}-\bar{X}\right)^{2}+\sum_{i=1}^{n}\left(Y_{i}-\bar{Y}\right)^{2}}\right] \\
& \quad+\left[\frac{\sqrt{n} \sum_{i=1}^{n}\left(X_{i}-\mu_{1}\right)\left(Y_{i}-\mu_{2}\right)}{\sum_{i=1}^{n}\left(X_{i}-\bar{X}\right)^{2}+\sum_{i=1}^{n}\left(Y_{i}-\bar{Y}\right)^{2}}-\frac{\sqrt{n} \sum_{i=1}^{n}\left(X_{i}-\mu_{1}\right)\left(Y_{i}-\mu_{2}\right)}{\sum_{i=1}^{n}\left(X_{i}-\mu_{1}\right)^{2}+\sum_{i=1}^{n}\left(Y_{i}-\mu_{2}\right)^{2}}\right]
\end{aligned}
$$

and factoring, we have

$$
\begin{aligned}
& \frac{\sqrt{n}}{2}\left(\hat{\rho}_{n}-\widetilde{\rho}_{n}\right) \\
& =\sqrt{n}\left[\frac{\sum_{i=1}^{n}\left(X_{i}-\bar{X}\right)\left(Y_{i}-\bar{Y}\right)-\sum_{i=1}^{n}\left(X_{i}-\mu_{1}\right)\left(Y_{i}-\mu_{2}\right)}{\sum_{i=1}^{n}\left(X_{i}-\bar{X}\right)^{2}+\sum_{i=1}^{n}\left(Y_{i}-\bar{Y}\right)^{2}}\right] \\
& \quad+\sqrt{n} \sum_{i=1}^{n}\left[\left(X_{i}-\mu_{1}\right)\left(Y_{i}-\mu_{2}\right)\right] \\
& \quad\left[\frac{1}{\sum_{i=1}^{n}\left(X_{i}-\bar{X}\right)^{2}+\sum_{i=1}^{n}\left(Y_{i}-\bar{Y}\right)^{2}}-\frac{1}{\sum_{i=1}^{n}\left(x_{i}-\mu_{1}\right)^{2}+\sum_{i=1}^{n}\left(y_{i}-\mu_{2}\right)^{2}}\right]
\end{aligned}
$$


thus, applying the equality proved above in Equation 2.39

$$
\begin{aligned}
\frac{\sqrt{n}}{2}\left(\hat{\rho}_{n}-\tilde{\rho}_{n}\right) & \frac{-n \sqrt{n}\left(\bar{X}-\mu_{1}\right)\left(\bar{Y}-\mu_{2}\right)}{=} \frac{\sqrt{n} \sum_{i=1}^{n}\left(X_{i}-\mu_{1}\right)\left(Y_{i}-\mu_{2}\right)}{\sum_{i=1}^{n}\left(X_{i}-\bar{X}\right)^{2}+\sum_{i=1}^{n}\left(Y_{i}-\bar{Y}\right)^{2}} \\
& -\frac{\left[\sum_{i=1}^{n}\left(X_{i}-\bar{X}\right)^{2}+\sum_{i=1}^{n}\left(Y_{i}-\bar{Y}\right)^{2}\right]\left[\sum_{i=1}^{n}\left(X_{i}-\mu_{1}\right)^{2}+\sum_{i=1}^{n}\left(Y_{i}-\mu_{2}\right)^{2}\right]}{\left.\left[\sum_{i=1}^{n}\left(X_{i}-\bar{X}\right)^{2}+\sum_{i=1}^{n}\left(Y_{i}-\bar{Y}\right)^{2}\right]-\left[\sum_{i=1}^{n}\left(X_{i}-\mu_{1}\right)^{2}+\sum_{i=1}^{n}\left(Y_{i}-\mu_{2}\right)^{2}\right]\right\}} \\
= & \frac{-n \sqrt{n}\left(\bar{X}-\mu_{1}\right)\left(\bar{Y}-\mu_{2}\right)}{\sum_{i=1}^{n}\left(X_{i}-\bar{X}\right)^{2}+\sum_{i=1}^{n}\left(Y_{i}-\bar{y}\right)^{2}} \\
& +\frac{\sqrt{n} \sum_{i=1}^{n}\left(X_{i}-\mu_{1}\right)\left(Y_{i}-\mu_{2}\right)}{\left[\sum_{i=1}^{n}\left(X_{i}-\bar{X}\right)^{2}+\sum_{i=1}^{n}\left(Y_{i}-\bar{Y}\right)^{2}\right]\left[\sum_{i=1}^{n}\left(X_{i}-\mu_{1}\right)^{2}+\sum_{i=1}^{n}\left(Y_{i}-\mu_{2}\right)^{2}\right]} \\
& {\left[\sqrt{n}\left(\bar{X}-\mu_{1}\right)^{2}+\sqrt{n}\left(\bar{Y}-\mu_{2}\right)^{2}\right] }
\end{aligned}
$$

Note that as $n \rightarrow \infty$

$$
\frac{1}{n} \sum_{i=1}^{n}\left(X_{i}-\bar{X}\right)=\frac{1}{n} \sum_{i=1}^{n}\left(X_{i}-\mu_{1}\right)^{2}-\left(\bar{X}-\mu_{1}\right)^{2} \rightarrow \sigma^{2}
$$

with probability one, and likewise

$$
\frac{1}{n} \sum_{i=1}^{n}\left(Y_{i}-\bar{Y}\right)=\frac{1}{n} \sum_{i=1}^{n}\left(Y_{i}-\mu_{1}\right)^{2}-\left(\bar{Y}-\mu_{1}\right)^{2} \rightarrow \sigma^{2}
$$

with probability one.

Thus the first term in (2.41)

$$
\frac{-n \sqrt{n}\left(\bar{X}-\mu_{1}\right)\left(\bar{Y}-\mu_{2}\right)}{\sum_{i=1}^{n}\left(X_{i}-\bar{X}\right)^{2}+\sum_{i=1}^{n}\left(Y_{i}-\bar{Y}\right)^{2}}=-\frac{\sqrt{n}\left(\bar{X}-\mu_{1}\right)\left(\bar{Y}-\mu_{2}\right)}{\frac{\sum_{i=1}^{n}\left(X_{i}-\bar{X}\right)^{2}+\sum_{i=1}^{n}\left(Y_{i}-\bar{Y}\right)^{2}}{n}} \rightarrow 0
$$

in probability by Result 2.2.3.

In the same way, it can be shown that as $n \rightarrow \infty$, the second term in (2.41) converges to zero in probability. 
Summarizing the above, we have shown that $\sqrt{n}\left(\rho_{n}-\widetilde{\rho}_{n}\right) \rightarrow 0$ in probability as $n \rightarrow \infty$. This further implies that the limiting distribution of $\sqrt{n}\left(\hat{\rho}_{n}-\rho\right)$ is the same as that of

$$
\sqrt{n}\left(\widetilde{\rho}_{n}-\rho\right)
$$

Lemma 2.2.6. As $n \rightarrow \infty$ it is true that

$$
\left(\sqrt{n}\left(\xi_{n}-\rho \sigma^{2}\right), \sqrt{n}\left(\eta_{n}-\sigma^{2}\right), \sqrt{n}\left(\zeta_{n}-\sigma^{2}\right)\right) \rightarrow N(0, \Sigma)
$$

in distribution, where

$$
\Sigma=\sigma^{4}\left(\begin{array}{ccc}
1+\rho^{2} & 2 \rho & 2 \rho \\
2 \rho & 2 & 2 \rho^{2} \\
2 \rho & 2 \rho^{2} & 2
\end{array}\right)
$$

and $\xi_{n}, \eta_{n}$ and $\zeta_{n}$ are three random variables define as follows:

$$
\begin{aligned}
\xi_{n} & =\frac{1}{n} \sum_{i=1}^{n}\left(X_{i}-\mu_{1}\right)\left(Y_{i}-\mu_{2}\right) \\
\eta_{n} & =\frac{1}{n} \sum_{i=1}^{n}\left(X_{i}-\mu_{1}\right)^{2} \\
\zeta_{n} & =\frac{1}{n} \sum_{i=1}^{n}\left(Y_{i}-\mu_{2}\right)^{2}
\end{aligned}
$$

Proof. The Multivariate Central Limit Theorem implies

$$
\left(\sqrt{n}\left(\xi_{n}-\rho \sigma^{2}\right), \sqrt{n}\left(\eta_{n}-\sigma^{2}\right), \sqrt{n}\left(\zeta_{n}-\sigma^{2}\right)\right) \rightarrow N(0, \Sigma)
$$

in distribution where $\Sigma$ is the covariance matrix of the random vector

$$
\left(\left(X_{i}-\mu_{1}\right)\left(Y_{i}-\mu_{2}\right),\left(X_{i}-\mu_{1}\right)^{2},\left(Y_{i}-\mu_{2}\right)^{2}\right)
$$

Therefore it is sufficient to obtain the matrix $\Sigma=\left(\sigma_{i j}\right)_{1 \leq i, j \leq 3}$.

It can be shown that 


$$
\begin{aligned}
& \sigma_{11}=\left(1+\rho^{2}\right) \sigma^{4} \quad \sigma_{12}=2 \rho^{2} \sigma^{4} \quad \sigma_{13}=2 \rho^{2} \sigma^{4} \\
& \sigma_{22}=2 \sigma^{4} \quad \sigma_{23}=2 \rho^{2} \sigma^{4} \\
& \sigma_{33}=2 \sigma^{4}
\end{aligned}
$$

and so,

$$
\Sigma=\sigma^{4}\left(\begin{array}{ccc}
1+\rho^{2} & 2 \rho & 2 \rho \\
2 \rho & 2 & 2 \rho^{2} \\
2 \rho & 2 \rho^{2} & 2
\end{array}\right)
$$

Theorem 2.2.7. As $n \rightarrow \infty$, it holds that

$$
\sqrt{n}\left(\hat{\rho}_{n}-\rho\right) \rightarrow N\left(0, \gamma^{2}\right)
$$

in distribution where $\gamma^{2}=\left(1-\rho^{2}\right)^{2}$.

Proof. According to Lemma 2.2.5, it suffices to show that as $n \rightarrow \infty$

$$
\sqrt{n}\left(\widetilde{\rho}_{n}-\rho\right) \rightarrow N\left(0, \gamma^{2}\right)
$$

in distribution.

Clearly $\widetilde{\rho}_{n}$ can be expressed in terms of $\xi_{n}, \eta_{n}, \zeta_{n}$ as

$$
\widetilde{\rho}_{n}=\frac{2 \sum_{i=1}^{n}\left(x_{i}-\mu_{1}\right)\left(y_{i}-\mu_{2}\right)}{\sum_{i=1}^{n}\left(x_{i}-\mu_{1}\right)^{2}+\sum_{i=1}^{n}\left(y_{i}-\mu_{1}\right)}=\frac{2 \xi_{n}}{\eta_{n}+\zeta_{n}}
$$

Define the function $g(u, v, w)$ by

$$
g(u, v, w)=\frac{2 u}{v+w}
$$

then obviously

$$
\widetilde{\rho}_{n}=g(u, v, w)
$$

It is easy to see that

$$
\left(\frac{\partial g}{\partial u}, \frac{\partial g}{\partial v}, \frac{\partial g}{\partial w}\right)=\left(\frac{2}{v+w}, \frac{-2 u}{(v+w)^{2}}, \frac{-2 u}{(v+w)^{2}}\right)
$$


Evaluating it at $u=\rho \sigma^{2}, v=\sigma^{2}, w=\sigma^{2}$ we have

$$
\begin{aligned}
\left(\frac{\partial g}{\partial u}, \frac{\partial g}{\partial v}, \frac{\partial g}{\partial w}\right)_{\left(\rho \sigma^{2}, \sigma^{2}, \sigma^{2}\right)} & =\left(\frac{1}{\sigma^{2}}, \frac{-\rho}{2 \sigma^{2}}, \frac{-\rho}{2 \sigma^{2}}\right) \\
& =\frac{1}{\sigma^{2}}\left(1,-\frac{\rho}{2},-\frac{\rho}{2}\right)
\end{aligned}
$$

By the delta method it follows that

$$
\sqrt{n}\left(g\left(\xi_{n}, \eta_{n}, \zeta_{n}\right)-g\left(\rho \sigma^{2}, \sigma^{2}, \sigma^{2}\right)\right) \rightarrow N\left(0, \gamma^{2}\right)
$$

in distribution where

$$
\begin{aligned}
\gamma^{2} & =\frac{1}{\sigma^{4}} \times\left(1,-\frac{\rho}{2},-\frac{\rho}{2}\right) \Sigma\left(\begin{array}{c}
1 \\
-\frac{\rho}{2} \\
-\frac{\rho}{2}
\end{array}\right) \\
& =\left(1,-\frac{\rho}{2},-\frac{\rho}{2}\right)\left(\begin{array}{rrr}
1+\rho^{2} & 2 \rho & 2 \rho \\
2 & 2 \rho^{2} \\
& 2
\end{array}\right)\left(\begin{array}{c}
1 \\
-\frac{\rho}{2} \\
-\frac{\rho}{2}
\end{array}\right) \\
= & \left(1-\rho^{2}\right)^{2} .
\end{aligned}
$$

Therefore, as $n \rightarrow \infty$

$$
\sqrt{n}\left(\widetilde{\rho}_{n}-\rho\right) \rightarrow N\left(0, \gamma^{2}\right)
$$

and consequently

$$
\sqrt{n}\left(\hat{\rho}_{n}-\rho\right) \rightarrow N\left(0, \gamma^{2}\right)
$$

in distribution

The result of Theorem 2.2.7 can be expressed as

$$
\hat{\rho}_{n} \sim N\left(\rho, \frac{\left(1-\rho^{2}\right)^{2}}{\sqrt{n}}\right)
$$

Notice that the variance of the distribution involves the parameter $\rho$. How can we find a distribution whose variance is parameter - free? In the next section we address this problem. 


\subsubsection{Variance Stabilizing transformation: The limiting dis- tribution of the $z$-transformation of $\hat{\rho}$}

The Variance Stabilizing Transformation is used when the constant variance assumption is violated; that is, when $\operatorname{Var}(W)$, where $W$ is a random variable, is a function of $E[W]=\mu$. In particular, the transformation assumes that $\operatorname{Var}(W)=g^{2}(\mu)$, where the function $g(\cdot)$ is known. The idea of it is to find a (differentiable) function $h(\cdot)$ so that the transformed random variable $h(W)$ will have a variance which is approximately independent of its mean $E[h(W)]$.

The delta method yields the following approximation:

$$
\operatorname{Var}(h(W)) \approx\left[h^{\prime}(\mu)\right]^{2} g^{2}(\mu)
$$

Then, the desired function $h(w)$ is the solution of the differential equation above. For our case, we need to find a function $h$ such that

$$
\sqrt{n}\left(h\left(\hat{\rho}_{n}\right)-h(\rho)\right) \sim N(0,1)
$$

therefore, applying the Variance Stabilizing transformation we have

$$
\left(h^{\prime}(\rho)\right)^{2}\left(1-\rho^{2}\right)^{2}=1
$$

from where, solving for $h$

$$
\begin{aligned}
\left.\int h^{\prime}(\rho)\right)^{2} d \rho & =\int \frac{1}{1-\rho^{2}} d \rho \\
h(\rho) & =\int \frac{1}{2(1-\rho)}+\frac{1}{2(1+\rho)} d \rho \\
h(\rho) & =-\frac{1}{2} \ln (1-\rho)+\frac{1}{2} \ln (1+\rho) \\
h(\rho) & =\frac{1}{2} \ln \left(\frac{1+\rho}{1-\rho}\right)
\end{aligned}
$$


Thus, we can conclude that

$$
\frac{\sqrt{n}}{2}\left(\ln \left(\frac{1+\hat{\rho}}{1-\hat{\rho}}\right)-\ln \left(\frac{1+\rho}{1-\rho}\right)\right) \sim N(0,1)
$$

The transformation express in Equation (2.44) is commonly called the "Fisher Transformation". 


\section{CHAPTER 3}

\section{TRIVARIATE NORMAL DISTRIBUTION}

In this chapter we introduce the Trivariate Normal Distribution and describe its probability density function. In particular, making the assumptions of common variances and common correlation coefficient, we derive its Maximum Likelihood Estimators and focus our study on the estimation of the correlation coefficient $\rho$.

Chapter 3 is developed as follows. In Section 3.1 we introduce the Trivariate Normal Distribution and its probability density function. In Section 3.2 we describe our particular case of the Covariance Matrix Symmetric Trivariate Normal Distribution, its probability density function in Section 3.2.1 and derive its estimators in Section 3.2.3 using the method of the Maximum Likelihood.

\subsection{General Discussion}

A three dimensional random variable $\left(Y_{1}, Y_{2}, Y_{3}\right)$ is said to have a Trivariate Normal Distribution if the joint probability distribution function can be represented in matrix form as

$$
f(\boldsymbol{y})=\frac{1}{(2 \pi)^{\frac{3}{2}}|\Sigma|^{\frac{1}{2}}} \exp \left\{-\frac{(\boldsymbol{y}-\boldsymbol{u})^{T} \Sigma^{-1}(\boldsymbol{y}-\boldsymbol{u})}{2}\right\}
$$

where $\boldsymbol{y}=\left(y_{1}, y_{2}, y_{3}\right)^{\prime}, \boldsymbol{\mu}=\left(\mu_{1}, \mu_{2}, \mu_{3}\right)^{\prime}, \Sigma$ is a $3 \times 3$, positive definite and symmetric variance-covariance matrix of the form

$$
\Sigma=\left(\begin{array}{lll}
\sigma_{11} & \sigma_{12} & \sigma_{13} \\
\sigma_{12} & \sigma_{22} & \sigma_{23} \\
\sigma_{13} & \sigma_{23} & \sigma_{33}
\end{array}\right)
$$


and $|\Sigma|>0$ is the determinant of $\Sigma$.

The density function of a Trivariate Normal Distribution can be written also as

$$
\begin{gathered}
f\left(y_{1}, y_{2}, y_{3}\right)=\frac{1}{(2 \pi)^{\frac{3}{2}} \sqrt{\sigma_{11} \sigma_{22} \sigma_{33}+2 \sigma_{12} \sigma_{13} \sigma_{23}-\sigma_{12}^{2} \sigma_{33}-\sigma_{13}^{2} \sigma_{22}-\sigma_{23}^{2} \sigma_{11}}} \\
\exp \left\{-\frac{1}{2\left(\sigma_{11} \sigma_{22} \sigma_{33}+2 \sigma_{12} \sigma_{13} \sigma_{23}-\sigma_{12}^{2} \sigma_{33}-\sigma_{13}^{2} \sigma_{22}-\sigma_{23}^{2} \sigma_{11}\right)} W\left(y_{1}, y_{2}, y_{3}\right)\right\}
\end{gathered}
$$

where

$$
\begin{array}{r}
W\left(y_{1}, y_{2}, y_{3}\right)=\left(y_{1}-\mu_{1}\right)^{2}\left(\sigma_{22} \sigma_{33}-\sigma_{23}^{2}\right)+\left(y_{2}-\mu_{2}\right)^{2}\left(\sigma_{11} \sigma_{33}-\sigma_{13}^{2}\right) \\
+\left(y_{3}-\mu_{3}\right)^{2}\left(\sigma_{11} \sigma_{22}-\sigma_{12}^{2}\right)+2\left[\left(y_{1}-\mu_{1}\right)\left(y_{2}-\mu_{2}\right)\right. \\
\left(\sigma_{13} \sigma_{23}-\sigma_{12} \sigma_{33}\right)+\left(y_{1}-\mu_{1}\right)\left(y_{2}-\mu_{2}\right)\left(\sigma_{13} \sigma_{23}-\sigma_{12} \sigma_{33}\right) \\
\left.+\left(y_{1}-\mu_{1}\right)\left(y_{2}-\mu_{2}\right)\left(\sigma_{13} \sigma_{23}-\sigma_{12} \sigma_{33}\right)\right]
\end{array}
$$

and provided that $\sigma_{11} \sigma_{22} \sigma_{33}+2 \sigma_{12} \sigma_{13} \sigma_{23}-\sigma_{12}^{2} \sigma_{33}-\sigma_{13}^{2} \sigma_{22}-\sigma_{23}^{2} \sigma_{11} \neq 0$.

\subsection{Covariance Matrix Symmetric}

We focus our study on a special form of the variance-covariance matrix $\Sigma$, a symmetric matrix where all variances are assumed to be equal and correlation coefficients too. It will be discussed below.

\subsubsection{Model}

For our study let us consider the case of equal variances, i.e. $\sigma_{11}=\sigma_{22}=\sigma_{33}=\sigma^{2}$ and equal correlation coefficient for each pair of variables, that is $\rho_{12}=\rho_{13}=\rho_{23}=$ $\rho$, thus the variance-covariance matrix $\Sigma$ has the form

$$
\Sigma=\left(\begin{array}{ccc}
\sigma^{2} & \sigma^{2} \rho & \sigma^{2} \rho \\
\sigma^{2} \rho & \sigma^{2} & \sigma^{2} \rho \\
\sigma^{2} \rho & \sigma^{2} \rho & \sigma^{2}
\end{array}\right)=\sigma^{2}\left(\begin{array}{ccc}
1 & \rho & \rho \\
\rho & 1 & \rho \\
\rho & \rho & 1
\end{array}\right)
$$


It can be shown that $|\Sigma|=\left(\sigma^{2}\right)^{3}\left(1+2 \rho^{3}-3 \rho^{2}\right)$ and thus

$$
\Sigma^{-1}=\frac{1}{\sigma^{2}\left(2 \rho^{3}-3 \rho^{2}+1\right)}\left(\begin{array}{ccc}
1-\rho^{2} & \rho^{2}-\rho & \rho^{2}-\rho \\
\rho^{2}-\rho & 1-\rho^{2} & \rho^{2}-\rho \\
\rho^{2}-\rho & \rho^{2}-\rho & 1-\rho^{2}
\end{array}\right)
$$

provided that $|\Sigma|=\left(\sigma^{2}\right)^{3}\left(2 \rho^{3}-3 \rho^{2}+1\right)=\left(\sigma^{2}\right)^{3}(2 \rho+1)(\rho-1)^{2} \neq 0$.

Therefore, the probability density function for this Trivaritate Normal Distribution is written as

$$
f\left(y_{1}, y_{2}, y_{3}\right)=\frac{1}{(2 \pi)^{\frac{3}{2}}\left(\sigma^{2}\right)^{\frac{3}{2}} \sqrt{2 \rho^{3}-3 \rho^{2}+1}} \exp \left\{-\frac{w^{*}}{2 \sigma^{2}\left(2 \rho^{2}-\rho-1\right)}\right\}
$$

where

$$
\begin{array}{r}
w^{*}=2 \rho\left[\left(y_{1}-\mu_{1}\right)\left(y_{2}-\mu_{2}\right)+\left(y_{1}-\mu_{1}\right)\left(y_{3}-\mu_{3}\right)+\left(y_{2}-\mu_{2}\right)\left(y_{3}-\mu_{3}\right)\right] \\
-(1+\rho)\left[\left(y_{1}-\mu_{1}\right)^{2}+\left(y_{2}-\mu_{2}\right)^{2}+\left(y_{3}-\mu_{3}\right)^{2}\right]
\end{array}
$$

and $-\frac{1}{2}<\rho<1$.

\subsubsection{Likelihood Function}

To obtain the likelihood function of the Covariance matrix Symmetric Trivariate Normal Distribution let us take a random sample of $n 3 \times 1$-vectors $\boldsymbol{y}_{1}, \boldsymbol{y}_{2}, \ldots, \boldsymbol{y}_{n}$, where $\boldsymbol{y}_{k}=\left(y_{k 1}, y_{k 2}, y_{k 3}\right)$ for $1 \leq k \leq n$.

Thus, the likelihood function $L\left(\boldsymbol{y} ; \boldsymbol{\mu}, \sigma^{2}, \rho\right)$ is

$$
\begin{aligned}
L\left(\boldsymbol{\mu}, \sigma^{2}, \rho ; \boldsymbol{y}_{1}, \boldsymbol{y}_{2}, \ldots, \boldsymbol{y}_{n}\right) & =\prod_{i=1}^{n} f\left(\boldsymbol{y}_{i} ; \boldsymbol{\mu}, \sigma^{2}, \rho\right) \\
& =\prod_{i=1}^{n} \frac{1}{(2 \pi)^{\frac{3}{2}}\left(\sigma^{2}\right)^{\frac{3}{2}} \sqrt{2 \rho^{3}-3 \rho^{2}+1}} \exp \left\{\frac{-w_{i}^{*}}{2 \sigma^{2}\left(2 \rho^{2}-\rho-1\right)}\right\}
\end{aligned}
$$


where

$$
\begin{aligned}
w_{i}^{*}= & 2 \rho\left[\left(y_{i 1}-\mu_{1}\right)\left(y_{i 2}-\mu_{2}\right)+\left(y_{i 1}-\mu_{1}\right)\left(y_{i 3}-\mu_{3}\right)+\left(y_{i 2}-\mu_{2}\right)\left(y_{i 3}-\mu_{3}\right)\right] \\
& -(1+\rho)\left[\left(y_{i 1}-\mu_{1}\right)^{2}+\left(y_{i 2}-\mu_{2}\right)^{2}+\left(y_{i 3}-\mu_{3}\right)^{2}\right]
\end{aligned}
$$

Taking $\tau=\sigma^{2}$, the likelihood function $L\left(\boldsymbol{\mu}, \sigma^{2}, \rho ; \boldsymbol{y}_{1}, \boldsymbol{y}_{2}, \ldots, \boldsymbol{y}_{n}\right)$ is

$$
L\left(\boldsymbol{\mu}, \sigma^{2}, \rho ; \boldsymbol{y}_{1}, \boldsymbol{y}_{2}, \ldots, \boldsymbol{y}_{n}\right)=\frac{1}{(2 \pi)^{\frac{3 n}{2}} \tau^{\frac{3 n}{2}}\left(2 \rho^{3}-3 \rho^{2}+1\right)^{\frac{n}{2}}} \exp \left\{\frac{-w^{* *}}{2 \tau\left(2 \rho^{2}-\rho-1\right)}\right\}
$$

where $\mu=\left(\mu_{1}, \mu_{2}, \mu_{3}\right)$ and

$$
\begin{aligned}
w^{* *}= & 2 \rho \sum_{i=1}^{n}\left[\left(y_{i 1}-\mu_{1}\right)\left(y_{i 2}-\mu_{2}\right)+\left(y_{i 1}-\mu_{1}\right)\left(y_{i 3}-\mu_{3}\right)+\left(y_{i 2}-\mu_{2}\right)\left(y_{i 3}-\mu_{3}\right)\right] \\
& -(1+\rho) \sum_{i=1}^{n}\left[\left(y_{i 1}-\mu_{1}\right)^{2}+\left(y_{i 2}-\mu_{2}\right)^{2}+\left(y_{i 3}-\mu_{3}\right)^{2}\right]
\end{aligned}
$$

and the natural logarithm of the same function

$$
\begin{aligned}
\ln L\left(\boldsymbol{\mu}, \sigma^{2}, \rho ; \boldsymbol{y}_{1}, \boldsymbol{y}_{2}, \ldots, \boldsymbol{y}_{n}\right)= & \frac{(1+\rho) \sum_{i=1}^{n} \sum_{j=1}^{3}\left(y_{i j}-\mu_{j}\right)^{2}}{2 \tau\left(2 \rho^{2}-\rho-1\right)} \\
& -\frac{2 \rho \sum_{i=1}^{n} \sum_{\substack{j, k=1 \\
j \neq k}}^{3}\left(y_{i j}-\mu_{j}\right)\left(y_{i k}-\mu_{k}\right)}{2 \tau\left(2 \rho^{2}-\rho-1\right)} \\
& -\ln (2 \pi)^{\frac{3 n}{2}}-\frac{3 n}{2} \ln \tau-\frac{n}{2} \ln \left(2 \rho^{3}-3 \rho^{2}+1\right)
\end{aligned}
$$

\subsubsection{Derivation of Maximum Likelihood Estimators}

Since $\mu_{1}, \mu_{2}, \mu_{3}, \tau$ and $\rho$ are unknown, we want to find the MLE's for these parameters, solving the system of equations

$$
\frac{\partial \ln L}{\partial \mu_{i}}=0, i=1,2,3 ; \quad \frac{\partial \ln L}{\partial \tau}=0, \frac{\partial \ln L}{\partial \rho}=0
$$


that is:

$$
\begin{aligned}
& \frac{\partial \ln L}{\partial \mu_{1}}= \frac{-2(1+\rho) \sum_{i=1}^{n}\left(y_{i 1}-\mu_{1}\right)+2 \rho \sum_{i=1}^{n}\left[\left(y_{i 2}-\mu_{2}\right)+\left(y_{i 3}-\mu_{3}\right)\right]}{2 \tau\left(2 \rho^{2}-\rho-1\right)}=0 \\
& \frac{\partial \ln L}{\partial \mu_{2}}= \frac{-2(1+\rho) \sum_{i=1}^{n}\left(y_{i 2}-\mu_{2}\right)+2 \rho \sum_{i=1}^{n}\left[\left(y_{i 1}-\mu_{1}\right)+\left(y_{i 3}-\mu_{3}\right)\right]}{2 \tau\left(2 \rho^{2}-\rho-1\right)}=0 \\
& \frac{\partial \ln L}{\partial \mu_{3}}= \frac{-2(1+\rho) \sum_{i=1}^{n}\left(y_{i 3}-\mu_{3}\right)+2 \rho \sum_{i=1}^{n}\left[\left(y_{i 1}-\mu_{1}\right)+\left(y_{i 2}-\mu_{2}\right)\right]}{2 \tau\left(2 \rho^{2}-\rho-1\right)}=0 \\
& \frac{\partial \ln L}{\partial \tau}=-\frac{(1+\rho) \sum_{i=1}^{n} \sum_{j=1}^{3}\left(y_{i j}-\mu_{j}\right)^{2}-2 \rho \sum_{i=1}^{n} \sum_{\substack{j, k=1 \\
j \neq k}}^{3}\left(y_{i j}-\mu_{j}\right)\left(y_{i k}-\mu_{k}\right)}{2 \tau^{2}\left(2 \rho^{2}-\rho-1\right)} \\
&-\frac{3 n}{2 \tau}=0 \\
& \frac{\partial \ln L}{\partial \rho}=\left(2 \rho^{2}-\rho-1\right) \psi\left[\sum_{i=1}^{n} \sum_{j=1}^{3}\left(y_{i j}-\mu_{j}\right)^{2}-2 \sum_{i=1}^{n} \sum_{j, k=1}^{3}\left(y_{i j}-\mu_{j}\right)\left(y_{i k}-\mu_{k}\right)\right] \\
&-\frac{n\left(6 \rho^{2}-6 \rho\right)}{2\left(2 \rho^{3}-3 \rho^{2}+1\right)}=0 \\
&-(4 \rho-1) \psi\left[(1+\rho) \sum_{i=1}^{n} \sum_{j=1}^{3}\left(y_{i j}-\mu_{j}\right)^{2}-2 \rho \sum_{i=1}^{3} \sum_{j, k=1}^{3}\left(y_{i j}-\mu_{j}\right)\left(y_{i k}-\mu_{k}\right)\right] \\
& j \neq k
\end{aligned}
$$

where

$$
\psi=\frac{2 \tau}{4 \tau^{2}\left(2 \rho^{2}-\rho-1\right)^{2}}
$$

In order to solve (3.15) - (3.18) for $\mu_{1}, \mu_{2}$ and $\mu_{3}$ we begin by multiplying equations (3.15),(3.16) and $(3.17)$ by $-\tau\left(2 \rho^{2}-\rho-1\right)$ and simplifying, we get

$$
\begin{aligned}
& (1+\rho) \sum_{i=1}^{n}\left(y_{i 1}-\mu_{1}\right)-\rho \sum_{i=1}^{n}\left[\left(y_{i 2}-\mu_{2}\right)+\left(y_{i 3}-\mu_{3}\right)\right]=0 \\
& (1+\rho) \sum_{i=1}^{n}\left(y_{i 2}-\mu_{2}\right)-\rho \sum_{i=1}^{n}\left[\left(y_{i 1}-\mu_{1}\right)+\left(y_{i 3}-\mu_{3}\right)\right]=0 \\
& (1+\rho) \sum_{i=1}^{n}\left(y_{i 3}-\mu_{3}\right)-\rho \sum_{i=1}^{n}\left[\left(y_{i 1}-\mu_{1}\right)+\left(y_{i 2}-\mu_{2}\right)\right]=0
\end{aligned}
$$


Now, adding equation (3.21) to equation (3.22) and simplifying the sum we get

$$
\left.\sum_{i=1}^{n}\left[\left(y_{i 2}-\mu_{2}\right)+\left(y_{i 3}-\mu_{3}\right)\right]\right)-2 \rho \sum_{i=1}^{n}\left(y_{i 1}-\mu_{1}\right)=0
$$

Multiplying (3.23) by $\rho$, adding it to equation (3.20) the we obtain

$$
\begin{aligned}
& \left(-2 \rho^{2}+\rho+1\right) \sum_{i=1}^{n}\left(y_{1 i}-\mu_{1}\right)=0 \\
& (2 \rho+1)(\rho-1) \sum_{i=1}^{n}\left(y_{1 i}-\mu_{1}\right)=0
\end{aligned}
$$

recall that $\rho \neq 1$ and $\rho \neq-\frac{1}{2}$, therefore it follows that

$$
\sum_{i=1}^{n}\left(y_{i 1}-\mu_{1}\right)=0
$$

which implies that the MLE $\hat{\mu}_{1}$ for $\mu_{1}$ is

$$
\hat{\mu}_{1}=\frac{\sum_{i=1}^{n} y_{i 1}}{n}=\bar{y}_{1}
$$

In similar way, we can obtain

$$
\hat{\mu}_{2}=\frac{\sum_{i=1}^{n} y_{i 2}}{n}=\bar{y}_{2}
$$

and

$$
\hat{\mu}_{3}=\frac{\sum_{i=1}^{n} y_{i 3}}{n}=\bar{y}_{3}
$$

Now, in order to find the estimators for $\rho$ and $\tau$, let us simplify equations (3.18) and equation (3.19) and substitute the estimators of $\mu_{1}, \mu_{2}$, and $\mu_{3}$ found in equations $(3.24),(3.25)$ and $(3.26)$ :

Multiplying equation (3.18) by $-2 \tau^{2}\left(2 \rho^{2}-\rho-1\right)$ we obtain

$$
\begin{array}{r}
(1+\rho) \sum_{i=1}^{n} \sum_{j=1}^{3}\left(y_{i j}-\bar{y}_{j}\right)^{2}-2 \rho \sum_{\substack { i=1 \\
\begin{subarray}{c}{j, k=1 \\
j \neq k{ i = 1 \\
\begin{subarray} { c } { j , k = 1 \\
j \neq k } }\end{subarray}}^{n}\left(y_{i j}-\bar{y}_{j}\right)\left(y_{i k}-\bar{y}_{k}\right) \\
+3 n \tau\left(2 \rho^{2}-\rho-1\right)=0
\end{array}
$$


and multiplying equation (3.19) by $2 \tau\left(2 \rho^{2}-\rho-1\right)^{2}$ and symplifying it we get

$$
\begin{array}{r}
\left(2 \rho^{2}-\rho-1\right)\left[\sum_{i=1}^{n} \sum_{j=1}^{3}\left(y_{i j}-\bar{y}_{j}\right)^{2}-2 \sum_{i=1}^{n} \sum_{\substack{j, k=1 \\
j \neq k}}^{3}\left(y_{i j}-\bar{y}_{j}\right)\left(y_{i k}-\bar{y}_{k}\right)\right] \\
-(4 \rho-1)\left[(1+\rho) \sum_{i=1}^{n} \sum_{j=1}^{3}\left(y_{i j}-\bar{y}_{j}\right)^{2}-2 \rho \sum_{\substack { i=1 \\
\begin{subarray}{c}{j, k=1 \\
j \neq k{ i = 1 \\
\begin{subarray} { c } { j , k = 1 \\
j \neq k } }\end{subarray}}^{3}\left(y_{i j}-\bar{y}_{j}\right)\left(y_{i k}-\bar{y}_{k}\right)\right] \\
-6 n \rho \tau\left(2 \rho^{2}-\rho-1\right)=0 .
\end{array}
$$

Grouping commom terms, the equation (3.28) will become

$$
\begin{array}{r}
{\left[\left(2 \rho^{2}-\rho-1\right)-(4 \rho-1)(1+\rho)\right] \sum_{i=1}^{n} \sum_{j=1}^{3}\left(y_{i j}-\bar{y}_{j}\right)^{2}} \\
+\left[-2\left(2 \rho^{2}-\rho-1\right)+2 \rho(4 \rho-1)\right] \sum_{\substack { i=1 \\
\begin{subarray}{c}{j, k=1 \\
j \neq k{ i = 1 \\
\begin{subarray} { c } { j , k = 1 \\
j \neq k } }\end{subarray}}^{3}\left(y_{i j}-\bar{y}_{j}\right)\left(y_{i k}-\bar{y}_{k}\right) \\
-6 n \rho \tau\left(2 \rho^{2}-\rho-1\right)=0
\end{array}
$$

and finally combining like terms we get

$$
\begin{array}{r}
-2 \rho(\rho+2) \sum_{i=1}^{n} \sum_{j=1}^{3}\left(y_{i j}-\bar{y}_{j}\right)^{2}+2\left(2 \rho^{2}+1\right) \sum_{\substack { i=1 \\
\begin{subarray}{c}{j, k=1 \\
j \neq k{ i = 1 \\
\begin{subarray} { c } { j , k = 1 \\
j \neq k } }\end{subarray}}^{n}\left(y_{i j}-\bar{y}_{j}\right)\left(y_{i k}-\bar{y}_{k}\right) \\
-6 n \rho \tau\left(2 \rho^{2}-\rho-1\right)=0
\end{array}
$$

Now, adding equations (3.30) and the product of equation (3.27) by $2 \rho$, we obtain

$$
-2 \rho \sum_{i=1}^{n} \sum_{j=1}^{3}\left(y_{i j}-\mu_{j}\right)^{2}+2 \sum_{i=1}^{n} \sum_{\substack{j, k=1 \\ j \neq k}}^{3}\left(y_{i j}-\mu_{j}\right)\left(y_{i k}-\mu_{k}\right)=0
$$

and solving it for $\rho$ we get

$$
\hat{\rho}=\frac{\sum_{i=1}^{n} \sum_{\substack{j, k=1 \\ j \neq k}}^{3}\left(y_{i j}-\bar{y}_{j}\right)\left(y_{i k}-\bar{y}_{k}\right)}{\sum_{i=1}^{n} \sum_{j=1}^{3}\left(y_{i j}-\bar{y}_{j}\right)^{2}}
$$


which is equivalent to

$$
\hat{\rho}=\frac{\sum_{i=1}^{n}\left[\left(y_{i 1}-\bar{y}_{1}\right)\left(y_{i 2}-\bar{y}_{2}\right)+\left(y_{i 1}-\bar{y}_{1}\right)\left(y_{i 3}-\bar{y}_{3}\right)+\left(y_{i 2}-\bar{y}_{2}\right)\left(y_{i 3}-\bar{y}_{3}\right)\right]}{\sum_{i=1}^{n}\left[\left(y_{i 1}-\bar{y}_{1}\right)^{2}+\left(y_{i 2}-\bar{y}_{2}\right)^{2}+\left(y_{i 3}-\bar{y}_{3}\right)^{2}\right]}
$$

Replacing $\rho$ in (3.27) by $\hat{\rho}$ in(3.33) and solving (??) for $\tau$, we obtain the MLE $\hat{\tau}$ for $\tau$

$$
\hat{\tau}=\frac{\sum_{i=1}^{n}\left[\left(y_{i 1}-\bar{y}_{1}\right)^{2}+\left(y_{i 2}-\bar{y}_{2}\right)^{2}+\left(y_{i 3}-\bar{y}_{3}\right)^{2}\right]}{3 n}
$$

and thus $\hat{\sigma}$ is

$$
\hat{\sigma}=\sqrt{\frac{\sum_{i=1}^{n}\left[\left(y_{i 1}-\bar{y}_{1}\right)^{2}+\left(y_{i 2}-\bar{y}_{2}\right)^{2}+\left(y_{i 3}-\bar{y}_{3}\right)^{2}\right]}{3 n}}
$$

\subsubsection{Properties of the MLE of $\rho$}

Theorem 3.2.1. It holds that $-\frac{1}{2}<\hat{\rho}<1$ with probability one.

Proof.

$$
\begin{gathered}
\hat{\rho} \geq-\frac{1}{2} \Leftrightarrow \frac{\sum_{i=1}^{n} \sum_{\substack{j, k=1 \\
j \neq k}}^{3}\left(y_{i j}-\bar{y}_{j}\right)\left(y_{i k}-\bar{y}_{k}\right)}{\sum_{i=1}^{n} \sum_{j=1}^{3}\left(y_{i j}-\bar{y}_{j}\right)^{2}} \geq-\frac{1}{2} \\
\Leftrightarrow 2 \sum_{i=1}^{n} \sum_{\substack{j, k=1 \\
j \neq k}}^{3}\left(y_{i j}-\bar{y}_{j}\right)\left(y_{i k}-\bar{y}_{k}\right) \geq-\sum_{i=1}^{n} \sum_{j=1}^{3}\left(y_{i j}-\bar{y}_{j}\right)^{2} \\
\Leftrightarrow \sum_{i=1}^{n} \sum_{j=1}^{3}\left(y_{i j}-\bar{y}_{j}\right)^{2}+2 \sum_{i=1}^{n} \sum_{\substack{j, k=1 \\
j \neq k}}^{3}\left(y_{i j}-\bar{y}_{j}\right)\left(y_{i k}-\bar{y}_{k}\right) \geq 0
\end{gathered}
$$




$$
\begin{aligned}
& \Leftrightarrow \sum_{i=1}^{n}\left[\left(y_{i 1}-\bar{y}_{1}\right)^{2}+\left(y_{i 2}-\bar{y}_{2}\right)^{2}+\left(y_{i 3}-\bar{y}_{3}\right)^{2}+2\left(y_{i 1}-\bar{y}_{1}\right)\left(y_{i 2}-\bar{y}_{2}\right)\right. \\
& \left.\quad+2\left(y_{i 1}-\bar{y}_{1}\right)\left(y_{i 3}-\bar{y}_{3}\right)+2\left(y_{i 2}-\bar{y}_{2}\right)\left(y_{i 3}-\bar{y}_{3}\right)\right] \geq 0 \\
& \Leftrightarrow \sum_{i=1}^{n}\left(\left(y_{i 1}-\bar{y}_{1}\right)+\left(y_{i 2}-\bar{y}_{2}\right)+\left(y_{i 3}-\bar{y}_{3}\right)\right)^{2} \geq 0
\end{aligned}
$$

which is true for all $y_{i j}, i=1, \ldots, n ; j=1,2,3$.

Now, let us prove that $\hat{\rho} \leq 1$ :

$$
\begin{aligned}
\hat{\rho} \leq 1 \Leftrightarrow & \frac{\sum_{i=1}^{n} \sum_{\substack{j, k=1 \\
j \neq k}}^{3}\left(y_{i j}-\bar{y}_{j}\right)\left(y_{i k}-\bar{y}_{k}\right)}{\sum_{i=1}^{n} \sum_{j=1}^{3}\left(y_{i j}-\bar{y}_{j}\right)^{2}} \leq 1 \\
\Leftrightarrow & \sum_{i=1}^{n} \sum_{\substack{j, k=1 \\
j \neq k}}^{3}\left(y_{i j}-\bar{y}_{j}\right)\left(y_{i k}-\bar{y}_{k}\right) \leq \sum_{i=1}^{n} \sum_{j=1}^{3}\left(y_{i j}-\bar{y}_{j}\right)^{2} \\
\Leftrightarrow & \sum_{i=1}^{n} \sum_{j=1}^{3}\left(y_{i j}-\bar{y}_{j}\right)^{2}-\sum_{i=1}^{n} \sum_{\substack{j, k=1 \\
j \neq k}}^{3}\left(y_{i j}-\bar{y}_{j}\right)\left(y_{i k}-\bar{y}_{k}\right) \geq 0 \\
\Leftrightarrow & \sum_{i=1}^{n}\left[2\left(y_{i 1}-\bar{y}_{1}\right)^{2}+2\left(y_{i 2}-\bar{y}_{2}\right)^{2}+2\left(y_{i 3}-\bar{y}_{3}\right)^{2}-2\left(y_{i 1}-\bar{y}_{1}\right)\left(y_{i 2}-\bar{y}_{2}\right)\right. \\
\Leftrightarrow & \left.-2\left(y_{i 1}-\bar{y}_{1}\right)\left(y_{i 3}-\bar{y}_{3}\right)-2\left(y_{i 2}-\bar{y}_{2}\right)\left(y_{i 3}-\bar{y}_{3}\right)\right] \geq 0 \\
& \sum_{i=1}^{n}\left[\left(\left(y_{i 1}-\bar{y}_{1}\right)-\left(y_{i 2}-\bar{y}_{2}\right)\right)^{2}+\left(\left(y_{i 1}-\bar{y}_{1}\right)-\left(y_{i 3}-\bar{y}_{3}\right)\right)^{2}\right. \\
& \left.+\left(\left(y_{i 2}-\bar{y}_{2}\right)-\left(y_{i 3}-\bar{y}_{3}\right)\right)^{2}\right] \geq 0
\end{aligned}
$$

which is also true for all $y_{i j}, i=1, \ldots, n ; j=1,2,3$.

Note that $P\left(\hat{\rho}=-\frac{1}{2}\right.$ or 1$)=0$, therefore, we can conclude that $-\frac{1}{2}<\hat{\rho}<1$ with probability one. 


\subsection{Sampling Distribution of $\hat{\rho}$ of Trivariate Normal Distri- bution}

In Section 3.2.3 we derived the maximum likelihood estimator $\hat{\rho}$ of the correlation coefficient $\rho$. Now we will derive its limiting distribution.

\subsubsection{The Limiting Distribution of $\hat{\rho}$}

Mi Mie has shown that under the covariance-matrix symmetric Trivariate Normal Distribution the MLE $\hat{\rho}_{n}$ of the correlation coefficient $\rho$ is asymptotically normal, that is

$$
\sqrt{n}\left(\hat{\rho_{n}}-\rho\right) \rightarrow N\left(0, \frac{(1-\rho)^{2}(1+2 \rho)^{2}}{3}\right)
$$

in distribution, but since the variance in the limiting distribution depends on the unknown parameter $\rho$, we want to find a function $h(\cdot)$ such that the limiting distribution of $\sqrt{n}\left(h\left(\hat{\rho_{n}}\right)-h(\rho)\right)$ will be parameter-free, that is

$$
\sqrt{n}\left(h\left(\hat{\rho_{n}}\right)-h(\rho)\right) \rightarrow N(0,1)
$$

For our purpose, we will use the Variance-Stabilizing Transformation in the next section.

\subsubsection{Variance-stabilizing transformation: The limiting dis- tribution of the $z$ - transformation of $\hat{\rho}$}

Using the Variance - Stabilizing transformation explained in Section 2.2.7, we have to find the function $h(\cdot)$ such that

$$
\sqrt{n}\left(h\left(\hat{\theta_{n}}\right)-h(\theta)\right) \rightarrow N\left(0,\left(h^{\prime}(\theta)^{2} \tau^{2}(\theta)\right)\right)
$$


which in our desired case $\left[h^{\prime}(\theta)\right]^{2} \tau^{2}(\theta)=1$ and where $\tau^{2}(\rho)=\frac{(1-\rho)^{2}(1+2 \rho)^{2}}{3}$.

Solving for $h^{\prime}(\rho)$ in the previous equation

$$
h^{\prime}(\rho)=\frac{\sqrt{3}}{(1-\rho)(1+2 \rho)}
$$

and thus

$$
\begin{aligned}
h(\rho) & =\int h^{\prime}(\rho) d \rho \\
& =\sqrt{3} \int \frac{1}{(1-\rho)(1+2 \rho)} d \rho \\
& =\sqrt{3} \int \frac{1}{3}\left(\frac{2}{1+2 \rho}+\frac{1}{1-\rho}\right) d \rho \\
& =\frac{1}{\sqrt{3}}[\ln (1+2 \rho)-\ln (1-\rho)] \\
& =\frac{1}{\sqrt{3}} \ln \left(\frac{1+2 \rho}{1-\rho}\right)
\end{aligned}
$$

Therefore, applying the previous result, we can conclude that

$$
\sqrt{\frac{n}{3}}\left(\ln \left(\frac{1+2 \hat{\rho}}{1-\hat{\rho}}\right)-\ln \left(\frac{1+2 \rho}{1-\rho}\right)\right) \rightarrow N(0,1)
$$

in distribution. 


\section{CHAPTER 4 \\ NUMERICAL STUDY ON THE CASE OF BIVARIATE NORMAL DISTRIBUTION}

In Chapter 4 a simulation study is conducted to compare the performance of the estimators $\hat{\rho}_{n}$ and $\hat{r}_{n}$ of the correlation coefficient in the case of equal variances for the Bivariate Normal Distribution discussed in Chapter 2.

We compare these two point estimators in Section 4.1.1 and the performance of those have been evaluated using the Bias and Mean Square Error (MSE) in Section 4.1.2. Since in Theorem 2.2.7 was discussed the limiting distribution of $\hat{\rho}$ and in Section 2.2.7 was presented a parameter-free distribution for $\hat{\rho}$, a study on the confidence intervals for $\rho$ are made in Section 4.2 and Test of Hypothesis in Section 4.3.

\subsection{Comparison between $\hat{r}_{n}$ and $\hat{\rho}_{n}$}

?? In Section ?? we conduct simulations to compare (a) the Pearson Correlation Coefficient $r_{n}$ defined in Equation (2.7) and (b) the MLE defined in the Equation (2.33).

Without loss of generality, we assume $\mu^{T}=(0,0)$ and $\sigma^{2}=4$. We picked seven different sample sizes $(n)$, with $n$ ranging from 20 to 50 in increments of 5 and the 19 values $\rho$ that ranges from -0.9 to 0.9 in 0.1 increments. The simulations were run 1000 times. 


\subsubsection{Comparison Point Estimators of the Correlation Co- efficient $\rho$}

Figure 4.1 shows the comparison of the true value of $\rho$ and the two estimators considered here: (a) The MLE $\hat{\rho}_{n}$ and (b) the Pearson Correlation Coefficient $r_{n}$. Table 4.1 shows the results of the simulation.

In this figure we can see that the value of the estimators are close to the true value of $\rho$; however, the Pearson Correlation Coeffient is closer to $\rho$ than the MLE except when $\rho=0$ where both are the same. Note also that as the sample size increases, the difference between the estimators and $\rho$ decreases. 
Table 4.1: Comparison Point Estimators

\begin{tabular}{|c|c|c|c|c|c|c|c|c|}
\hline & \multirow{2}{*}{ Rho } & \multicolumn{7}{|c|}{ SAMPLE SIZE } \\
\hline & & 20 & 25 & 30 & 35 & 40 & 45 & 50 \\
\hline \multirow{19}{*}{$\sqrt{0}$} & -0.9 & -0.8910 & -0.8929 & -0.8934 & -0.8943 & -0.8955 & -0.8957 & -0.8967 \\
\hline & -0.8 & -0.7830 & -0.7886 & -0.7895 & -0.7919 & -0.7922 & -0.7929 & -0.7938 \\
\hline & -0.7 & -0.6810 & -0.6845 & -0.6881 & -0.6902 & -0.6892 & -0.6907 & -0.6908 \\
\hline & -0.6 & -0.5778 & -0.5853 & -0.5869 & -0.5877 & -0.5903 & -0.5919 & -0.5921 \\
\hline & -0.5 & -0.4795 & -0.4842 & -0.4877 & -0.4898 & -0.4919 & -0.4913 & -0.4938 \\
\hline & -0.4 & -0.3861 & -0.3866 & -0.3880 & -0.3916 & -0.3858 & -0.3909 & -0.3944 \\
\hline & -0.3 & -0.2871 & -0.2874 & -0.2914 & -0.2917 & -0.2933 & -0.2944 & -0.2944 \\
\hline & -0.2 & -0.1876 & -0.1927 & -0.1928 & -0.1938 & -0.1994 & -0.1952 & -0.1971 \\
\hline & -0.1 & -0.0947 & -0.0977 & -0.0959 & -0.0957 & -0.0974 & -0.0975 & -0.0968 \\
\hline & 0 & 0.0019 & 0.0018 & -0.0030 & -0.0016 & -0.0015 & 0.0025 & -0.0009 \\
\hline & 0.1 & 0.0952 & 0.0940 & 0.0957 & 0.0994 & 0.0974 & 0.0992 & 0.0983 \\
\hline & 0.2 & 0.1913 & 0.1887 & 0.1945 & 0.1955 & 0.1970 & 0.1932 & 0.1964 \\
\hline & 0.3 & 0.2837 & 0.2879 & 0.2918 & 0.2918 & 0.2948 & 0.2930 & 0.2948 \\
\hline & 0.4 & 0.3792 & 0.3861 & 0.3894 & 0.3915 & 0.3909 & 0.3931 & 0.3942 \\
\hline & 0.5 & 0.4793 & 0.4847 & 0.4878 & 0.4875 & 0.4909 & 0.4925 & 0.4935 \\
\hline & 0.6 & 0.5828 & 0.5821 & 0.5870 & 0.5900 & 0.5905 & 0.5906 & 0.5917 \\
\hline & 0.7 & 0.6813 & 0.6859 & 0.6866 & 0.6881 & 0.6903 & 0.6926 & 0.6929 \\
\hline & 0.8 & 0.7846 & 0.7879 & 0.7896 & 0.7915 & 0.7922 & 0.7936 & 0.7941 \\
\hline & 0.9 & 0.8902 & 0.8923 & 0.8935 & 0.8948 & 0.8957 & 0.8956 & 0.8964 \\
\hline \multirow{19}{*}{ อ } & -0.9 & -0.8959 & -0.8967 & -0.8965 & -0.8969 & -0.8978 & -0.8977 & -0.8984 \\
\hline & -0.8 & -0.7908 & -0.7946 & -0.7946 & -0.7963 & -0.7959 & -0.7963 & -0.7968 \\
\hline & -0.7 & -0.6904 & -0.6920 & -0.6943 & -0.6955 & -0.6939 & -0.6947 & -0.6944 \\
\hline & -0.6 & -0.5875 & -0.5930 & -0.5935 & -0.5934 & -0.5952 & -0.5961 & -0.5960 \\
\hline & -0.5 & -0.4889 & -0.4918 & -0.4940 & -0.4951 & -0.4966 & -0.4956 & -0.4976 \\
\hline & -0.4 & -0.3944 & -0.3931 & -0.3938 & -0.3966 & -0.3900 & -0.3946 & -0.3977 \\
\hline & -0.3 & -0.2937 & -0.2928 & -0.2959 & -0.2956 & -0.2966 & -0.2975 & -0.2970 \\
\hline & -0.2 & -0.1923 & -0.1965 & -0.1959 & -0.1966 & -0.2018 & -0.1973 & -0.1989 \\
\hline & -0.1 & -0.0970 & -0.0997 & -0.0974 & -0.0971 & -0.0987 & -0.0986 & -0.0977 \\
\hline & 0 & 0.0021 & 0.0019 & -0.0030 & -0.0016 & -0.0015 & 0.0025 & -0.0010 \\
\hline & 0.1 & 0.0976 & 0.0960 & 0.0973 & 0.1008 & 0.0987 & 0.1003 & 0.0993 \\
\hline & 0.2 & 0.1960 & 0.1924 & 0.1977 & 0.1982 & 0.1994 & 0.1953 & 0.1983 \\
\hline & 0.3 & 0.2905 & 0.2933 & 0.2963 & 0.2956 & 0.2982 & 0.2961 & 0.2975 \\
\hline & 0.4 & 0.3871 & 0.3927 & 0.3950 & 0.3964 & 0.3951 & 0.3968 & 0.3975 \\
\hline & 0.5 & 0.4885 & 0.4923 & 0.4941 & 0.4929 & 0.4956 & 0.4968 & 0.4973 \\
\hline & 0.6 & 0.5927 & 0.5901 & 0.5937 & 0.5956 & 0.5954 & 0.5948 & 0.5956 \\
\hline & 0.7 & 0.6906 & 0.6932 & 0.6928 & 0.6933 & 0.6949 & 0.6967 & 0.6966 \\
\hline & 0.8 & 0.7923 & 0.7940 & 0.7946 & 0.7957 & 0.7959 & 0.7970 & 0.7972 \\
\hline & 0.9 & 0.8950 & 0.8961 & 0.8966 & 0.8974 & 0.8979 & 0.8976 & 0.8982 \\
\hline
\end{tabular}



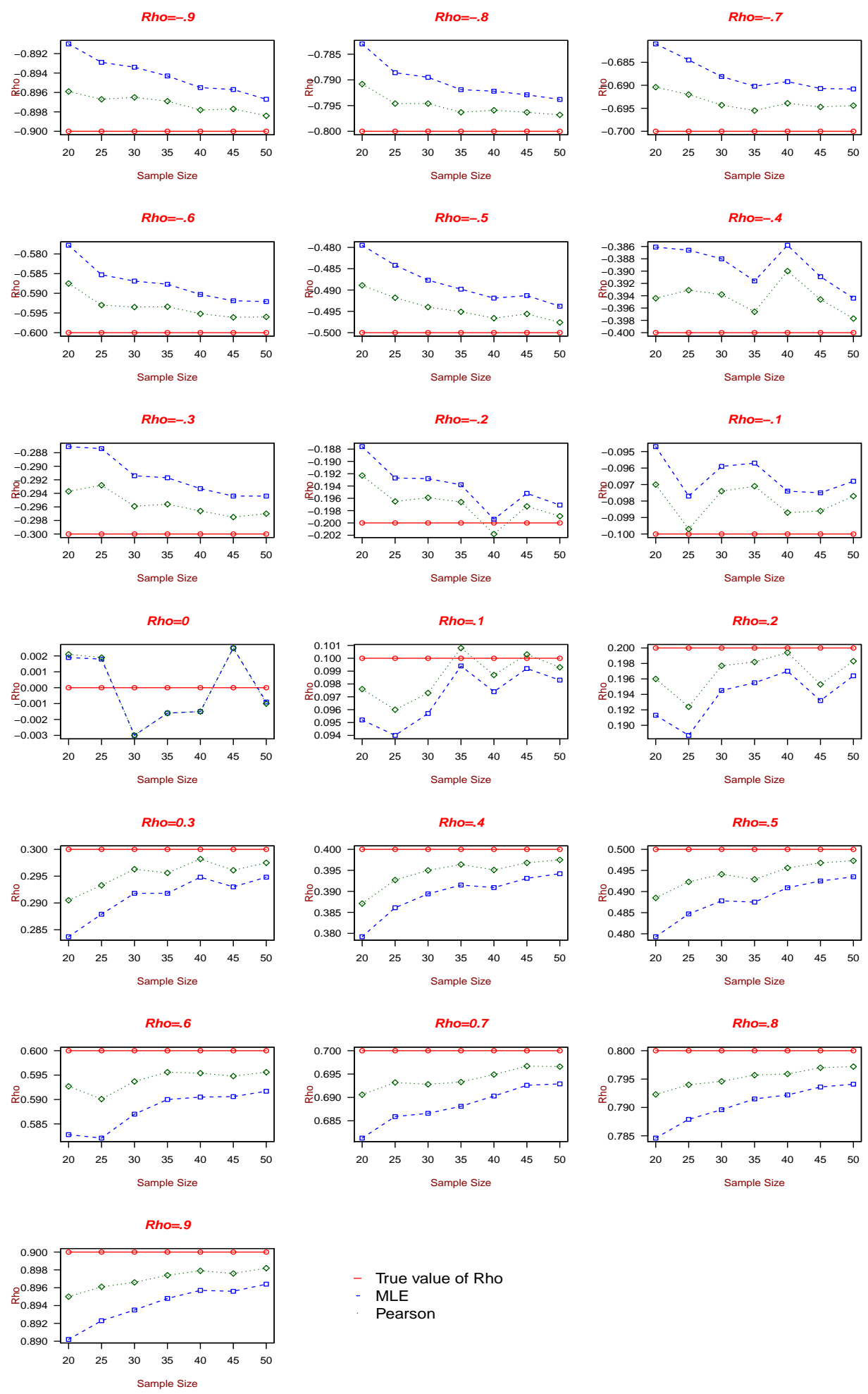

Figure 4.1: Comparison Point Estimators of Rho 


\subsubsection{Study on the Performance of the Estimators}

In order to compare the MLE and Pearson Correlation Coefficient, we evaluate two properties of the estimators: The Bias and the Mean Square Error.

In Table 4.2 and in Figure 4.2, the bias of both estimators are shown. The Pearson Correlation Coefficient always has smaller bias than the MLE except when $\rho=0$. In Figure 4.3 the absolute value of the Bias can be observed. From this Figure we can see that the absolute value of the bias decreases as the sample size increases.

Since the Bias is not enough to compare the performance of both estimators, the MSE of the estimators were found. Table 4.3 describes those values and are represented in Figure 4.4. It can be observed that there is not a noticeable difference on the MSE of the MLE compared to the Pearson Correlation Coefficient. 
Table 4.2: Comparison Bias Estimators

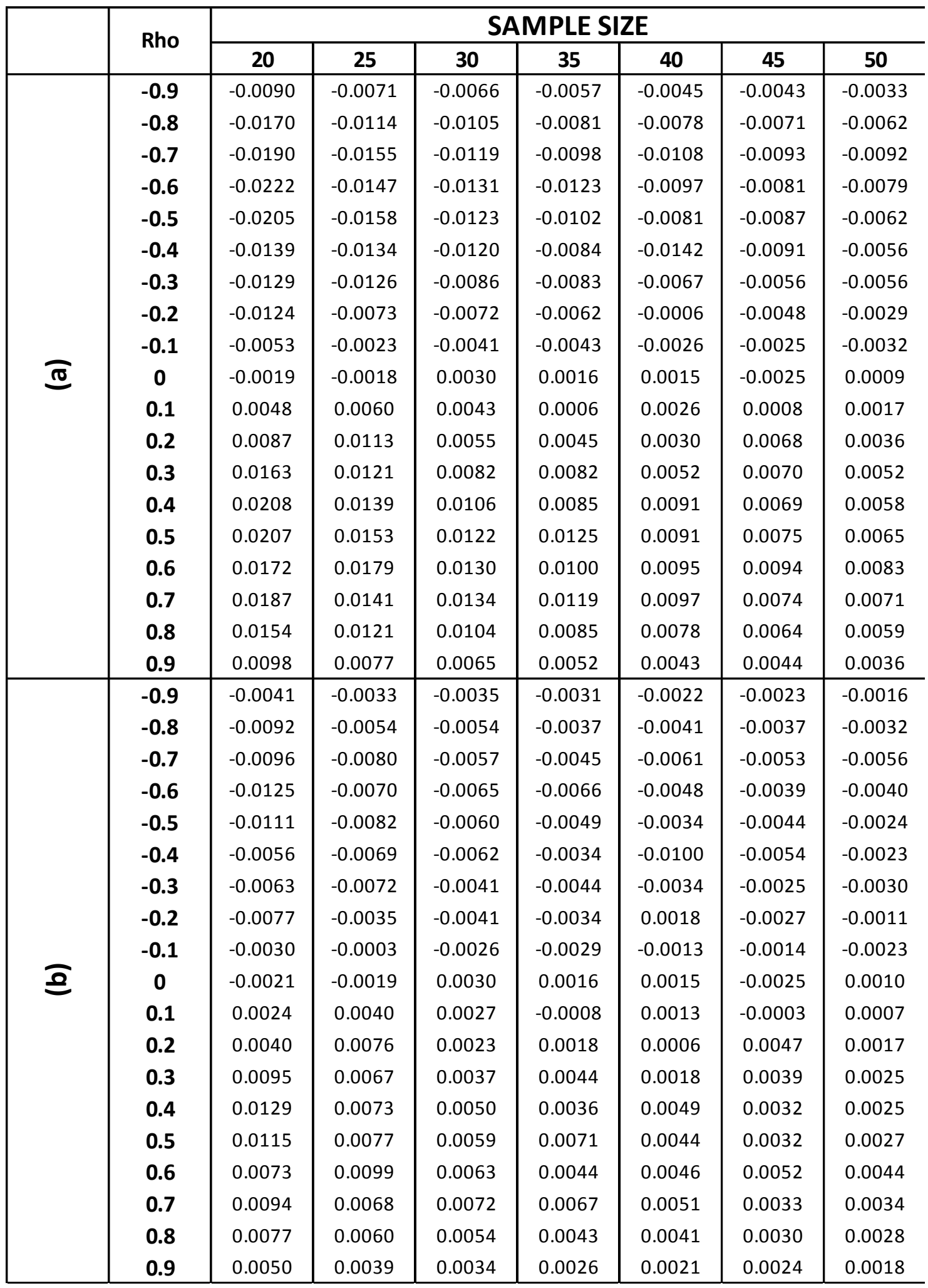



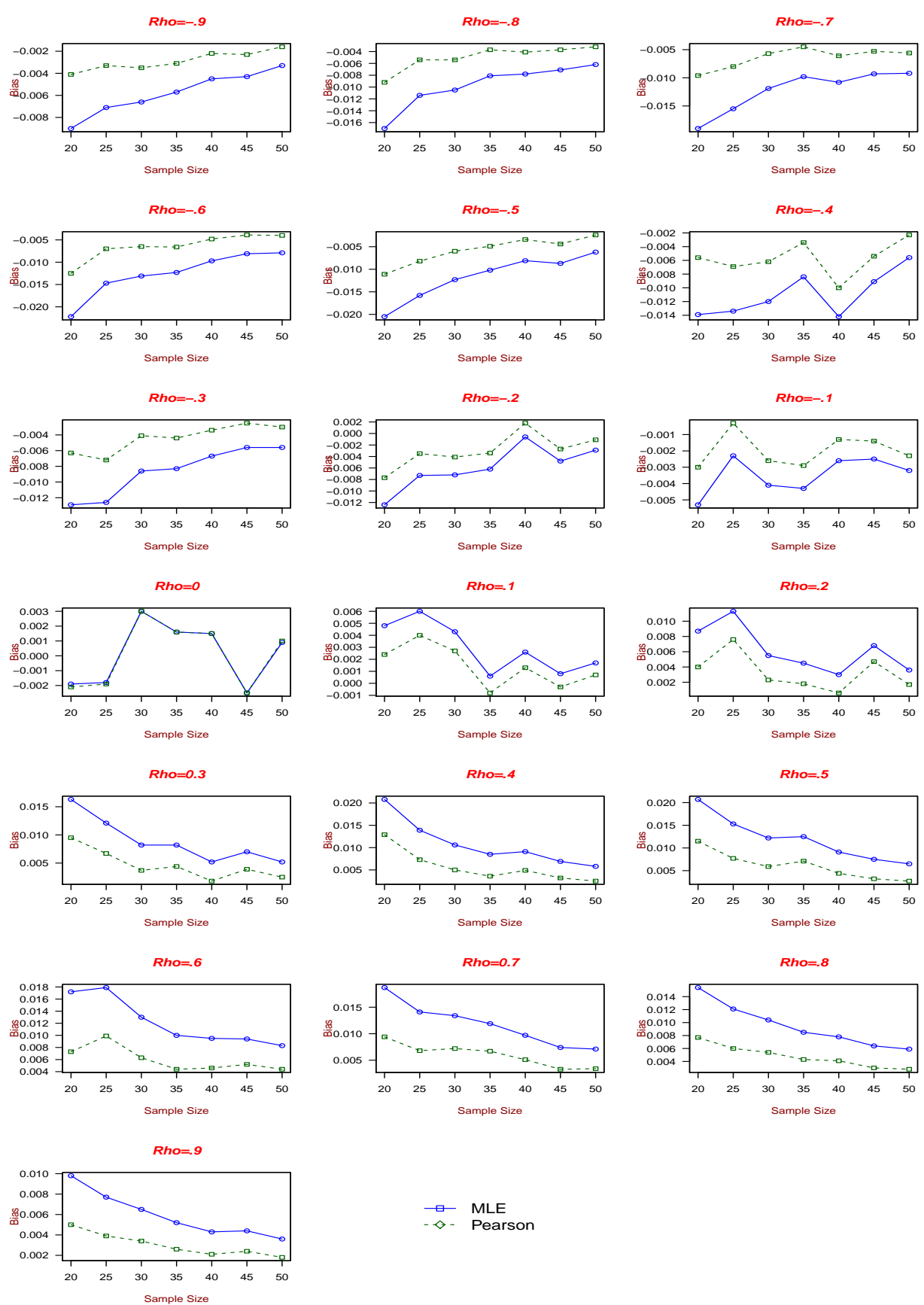

Figure 4.2: Comparison Bias of Point Estimators 
Rho $=-.9$

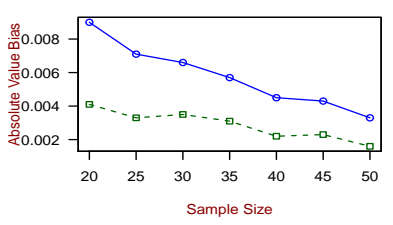

Rho $=-.6$

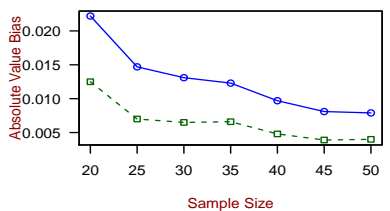

Rho $=-3$
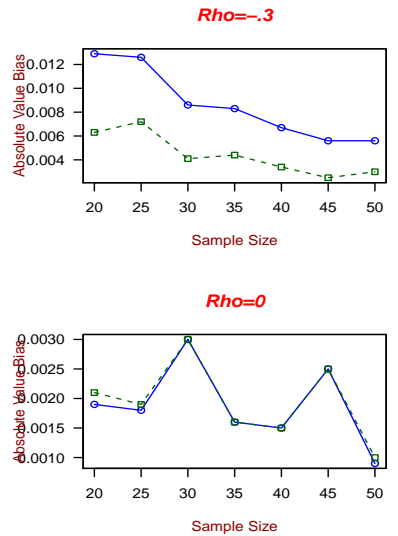

$R h o=0.3$

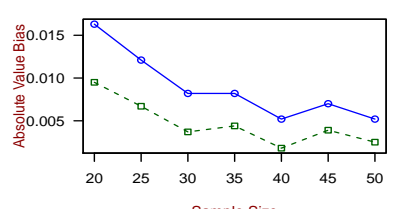

Rho $=.6$

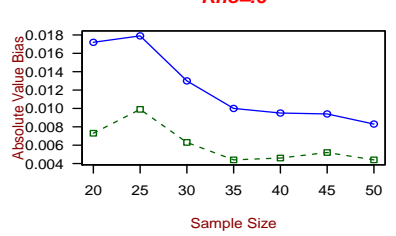

Rho $=.9$

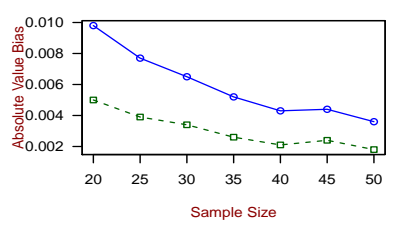

$R h o=-.8$

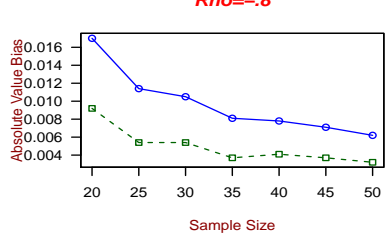

$R h o=-.5$

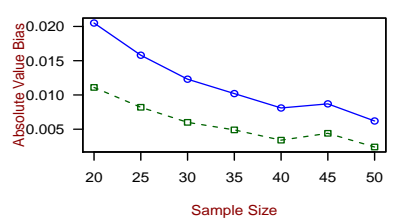

Rho $=-.2$

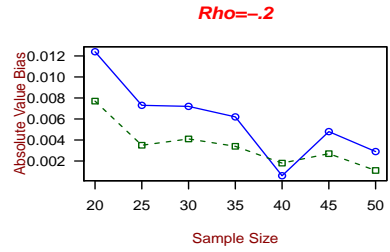

Rho $=.1$

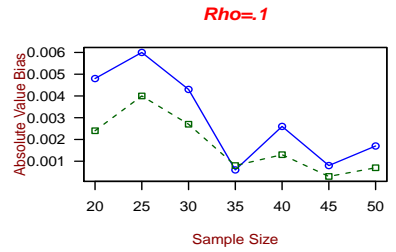

Rho $=.4$

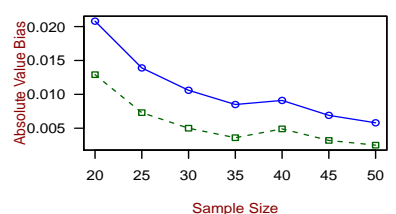

Rho $=0.7$

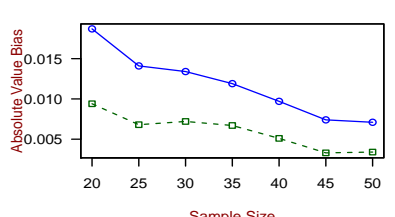

Sample Size

๑- MLE

$-\diamond$ - Pearson

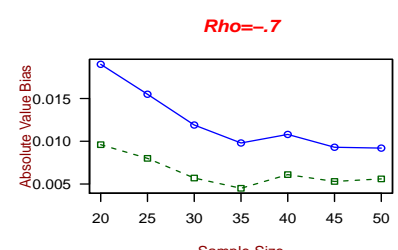

Rho $=-.4$

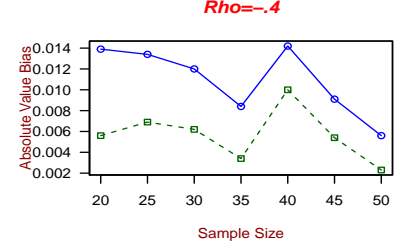

Rho $=-.1$
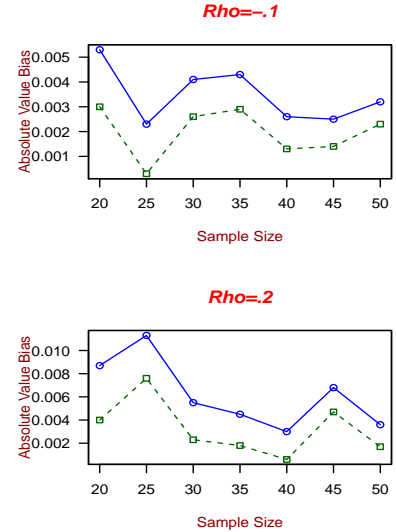

Rho $=.5$

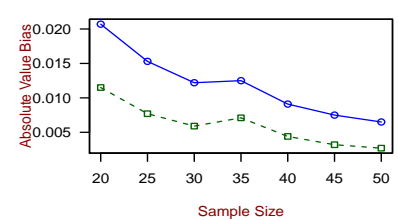

$R h o=.8$

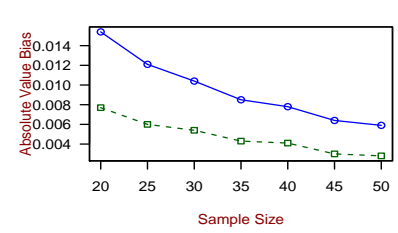

Figure 4.3: Comparison Absolute Value of Bias 
Table 4.3: Comparison MSE Estimators

\begin{tabular}{|c|c|c|c|c|c|c|c|c|}
\hline & \multirow{2}{*}{ Rho } & \multicolumn{7}{|c|}{ SAMPLE SIZE } \\
\hline & & 20 & 25 & 30 & 35 & 40 & 45 & 50 \\
\hline \multirow{19}{*}{$\sqrt{0}$} & -0.9 & 0.0026 & 0.0019 & 0.0016 & 0.0013 & 0.0011 & 0.0010 & 0.0008 \\
\hline & -0.8 & 0.0086 & 0.0064 & 0.0052 & 0.0043 & 0.0038 & 0.0032 & 0.0029 \\
\hline & -0.7 & 0.0162 & 0.0122 & 0.0097 & 0.0081 & 0.0073 & 0.0066 & 0.0059 \\
\hline & -0.6 & 0.0240 & 0.0186 & 0.0159 & 0.0129 & 0.0108 & 0.0098 & 0.0086 \\
\hline & -0.5 & 0.0313 & 0.0246 & 0.0200 & 0.0162 & 0.0146 & 0.0128 & 0.0119 \\
\hline & -0.4 & 0.0366 & 0.0308 & 0.0246 & 0.0205 & 0.0189 & 0.0160 & 0.0143 \\
\hline & -0.3 & 0.0429 & 0.0346 & 0.0279 & 0.0240 & 0.0209 & 0.0190 & 0.0170 \\
\hline & -0.2 & 0.0463 & 0.0367 & 0.0309 & 0.0267 & 0.0231 & 0.0211 & 0.0186 \\
\hline & -0.1 & 0.0485 & 0.0393 & 0.0331 & 0.0278 & 0.0243 & 0.0221 & 0.0193 \\
\hline & 0 & 0.0493 & 0.0389 & 0.0333 & 0.0286 & 0.0252 & 0.0221 & 0.0202 \\
\hline & 0.1 & 0.0490 & 0.0391 & 0.0326 & 0.0284 & 0.0243 & 0.0218 & 0.0201 \\
\hline & 0.2 & 0.0466 & 0.0372 & 0.0313 & 0.0265 & 0.0231 & 0.0206 & 0.0187 \\
\hline & 0.3 & 0.0430 & 0.0331 & 0.0277 & 0.0240 & 0.0212 & 0.0186 & 0.0166 \\
\hline & 0.4 & 0.0385 & 0.0303 & 0.0245 & 0.0209 & 0.0183 & 0.0165 & 0.0147 \\
\hline & 0.5 & 0.0312 & 0.0239 & 0.0203 & 0.0174 & 0.0151 & 0.0130 & 0.0118 \\
\hline & 0.6 & 0.0235 & 0.0185 & 0.0152 & 0.0127 & 0.0109 & 0.0097 & 0.0088 \\
\hline & 0.7 & 0.0157 & 0.0119 & 0.0100 & 0.0084 & 0.0073 & 0.0063 & 0.0058 \\
\hline & 0.8 & 0.0085 & 0.0068 & 0.0053 & 0.0044 & 0.0037 & 0.0032 & 0.0029 \\
\hline & 0.9 & 0.0026 & 0.0020 & 0.0016 & 0.0013 & 0.0011 & 0.0010 & 0.0008 \\
\hline \multirow{19}{*}{$\underline{2}$} & -0.9 & 0.0024 & 0.0018 & 0.0015 & 0.0013 & 0.0011 & 0.0009 & 0.0008 \\
\hline & -0.8 & 0.0082 & 0.0062 & 0.0051 & 0.0042 & 0.0038 & 0.0032 & 0.0029 \\
\hline & -0.7 & 0.0160 & 0.0119 & 0.0096 & 0.0080 & 0.0071 & 0.0065 & 0.0058 \\
\hline & -0.6 & 0.0240 & 0.0185 & 0.0159 & 0.0129 & 0.0107 & 0.0098 & 0.0086 \\
\hline & -0.5 & 0.0319 & 0.0249 & 0.0202 & 0.0164 & 0.0147 & 0.0129 & 0.0119 \\
\hline & -0.4 & 0.0377 & 0.0314 & 0.0251 & 0.0209 & 0.0191 & 0.0162 & 0.0145 \\
\hline & -0.3 & 0.0446 & 0.0357 & 0.0287 & 0.0245 & 0.0213 & 0.0193 & 0.0172 \\
\hline & -0.2 & 0.0484 & 0.0381 & 0.0318 & 0.0274 & 0.0237 & 0.0215 & 0.0189 \\
\hline & -0.1 & 0.0511 & 0.0410 & 0.0342 & 0.0286 & 0.0249 & 0.0225 & 0.0197 \\
\hline & 0 & 0.0519 & 0.0405 & 0.0345 & 0.0294 & 0.0259 & 0.0226 & 0.0206 \\
\hline & 0.1 & 0.0515 & 0.0407 & 0.0338 & 0.0293 & 0.0249 & 0.0222 & 0.0205 \\
\hline & 0.2 & 0.0488 & 0.0385 & 0.0323 & 0.0272 & 0.0237 & 0.0210 & 0.0191 \\
\hline & 0.3 & 0.0448 & 0.0341 & 0.0284 & 0.0245 & 0.0216 & 0.0190 & 0.0168 \\
\hline & 0.4 & 0.0395 & 0.0310 & 0.0250 & 0.0213 & 0.0186 & 0.0167 & 0.0149 \\
\hline & 0.5 & 0.0317 & 0.0241 & 0.0205 & 0.0175 & 0.0152 & 0.0131 & 0.0118 \\
\hline & 0.6 & 0.0235 & 0.0184 & 0.0151 & 0.0127 & 0.0109 & 0.0097 & 0.0088 \\
\hline & 0.7 & 0.0154 & 0.0117 & 0.0098 & 0.0082 & 0.0073 & 0.0062 & 0.0057 \\
\hline & 0.8 & 0.0081 & 0.0065 & 0.0052 & 0.0042 & 0.0036 & 0.0032 & 0.0028 \\
\hline & 0.9 & 0.0024 & 0.0018 & 0.0015 & 0.0012 & 0.0011 & 0.0009 & 0.0008 \\
\hline
\end{tabular}



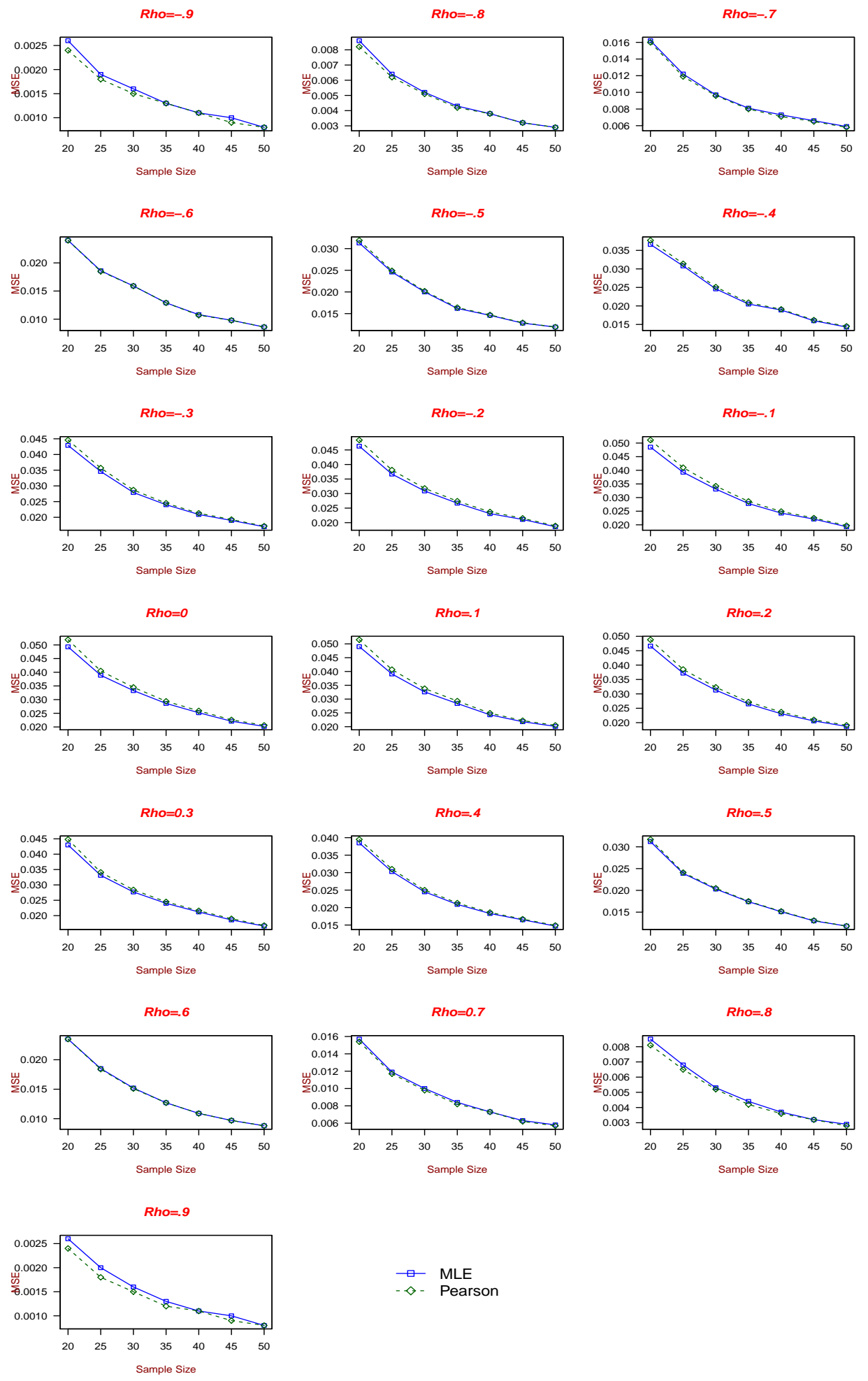

$\square \quad$ MLE

$-\diamond$ - Pearson

Figure 4.4: Comparison MSE 


\subsection{Confidence Interval for $\rho$}

As noted in Theorem 2.2.7, $\hat{\rho}_{n} \rightarrow N\left(\rho, \frac{\left(1-\rho^{2}\right)^{2}}{n}\right)$ in distribution; however, since the variance of it depends on $\rho$, a variance stabilizing transformation was used in Section 2.2.7 and it was found that $\frac{1}{2} \ln \left(\frac{1+\hat{\rho}_{n}}{1-\hat{\rho}_{n}}\right) \sim N\left(\frac{1}{2} \ln \left(\frac{1+\rho}{1-\rho}\right), \frac{1}{n}\right)$.

Using the previous two distributions, a $(1-\alpha) 100 \%$ Confidence Interval for $\rho$ can be found as follow:

$$
\left(\hat{\rho}-z_{\alpha / 2} \frac{1-\rho^{2}}{\sqrt{n}}, \hat{\rho}+z_{\alpha / 2} \frac{1-\rho^{2}}{\sqrt{n}}\right)
$$

or

$$
\left(\frac{e^{2 l}-1}{e^{2 l}+1}, \frac{e^{2 u}-1}{e^{2 u}+1}\right)
$$

respectively, where $l=\frac{1}{2} \ln \left(\frac{1+\hat{\rho}_{n}}{1-\hat{\rho}_{n}}\right)-z_{\alpha / 2} \frac{1}{\sqrt{n}}$ and $u=\frac{1}{2} \ln \left(\frac{1+\hat{\rho}_{n}}{1-\hat{\rho}_{n}}\right)+z_{\alpha / 2} \frac{1}{\sqrt{n}} ;$ however the desired confidence level is not attained using these intervals. Therefore, to achieve the $(1-\alpha) 100 \%$ level of confidence, a study on the denominators of the sampling errors is performed.

\subsubsection{Study on the Denominator for the Confidence Inter- vals}

Our overaching goal is to find a $95 \%$ confidence interval for $\rho$. For this purpose, the coverage probability of the intervals

$$
\left(\hat{\rho}-z_{\alpha / 2} \frac{1-\hat{\rho}^{2}}{\sqrt{n-k}}, \hat{\rho}+z_{\alpha / 2} \frac{1-\hat{\rho}^{2}}{\sqrt{n-k}}\right)
$$

for $k=0,1,2,3,4,5$ is calculated and recorded in Tables 4.4-4.5 and 4.6-4.7 where the MLE and Pearson Correlation Coefficient are used, respectively. 


\begin{tabular}{|c|c|c|c|c|c|c|c|c|c|c|c|c|c|c|c|}
\hline \multirow[b]{2}{*}{ RHO } & \multirow[b]{2}{*}{$\begin{array}{c}\text { SAMPLE } \\
\text { SIZE } \\
\end{array}$} & \multicolumn{6}{|c|}{ DENOMINADOR } & \multirow[b]{2}{*}{ RHO } & \multirow[b]{2}{*}{$\begin{array}{c}\text { SAMPLE } \\
\text { SIZE } \\
\end{array}$} & \multicolumn{6}{|c|}{ DENOMINADOR } \\
\hline & & $n$ & $n-1$ & $\mathrm{n}-\mathbf{2}$ & $n-3$ & $n-4$ & $n-5$ & & & $\mathbf{n}$ & $n-1$ & $n-2$ & $\mathbf{n}-\mathbf{3}$ & $n-4$ & $n-5$ \\
\hline \multirow{7}{*}{-0.9} & 20 & 0.9075 & 0.913 & 0.9184 & 0.923 & 0.9267 & 0.9322 & \multirow{7}{*}{-0.2} & 20 & 0.9093 & 0.9147 & 0.9208 & 0.9276 & 0.935 & 0.9414 \\
\hline & 25 & 0.9204 & 0.9241 & 0.9268 & 0.9307 & 0.9346 & 0.938 & & 25 & 0.9199 & 0.9257 & 0.9309 & 0.9354 & 0.9411 & 0.9461 \\
\hline & 30 & 0.9253 & 0.9277 & 0.9311 & 0.9347 & 0.9379 & 0.9413 & & 30 & 0.9242 & 0.9296 & 0.9354 & 0.9387 & 0.9433 & 0.947 \\
\hline & 35 & 0.9243 & 0.9273 & 0.9299 & 0.9322 & 0.935 & 0.9389 & & 35 & 0.9293 & 0.9329 & 0.9357 & 0.9382 & 0.9423 & 0.9455 \\
\hline & 40 & 0.9286 & 0.9316 & 0.9342 & 0.9358 & 0.9398 & 0.9431 & & 40 & 0.9272 & 0.9301 & 0.9325 & 0.9359 & 0.9384 & 0.9417 \\
\hline & 45 & 0.9332 & 0.9342 & 0.9371 & 0.9387 & 0.9414 & 0.9439 & & 45 & 0.9282 & 0.9303 & 0.9334 & 0.9368 & 0.9386 & 0.9422 \\
\hline & 50 & 0.9361 & 0.9377 & 0.9401 & 0.9431 & 0.9443 & 0.946 & & 50 & 0.9336 & 0.9356 & 0.9372 & 0.9399 & 0.9428 & 0.9459 \\
\hline \multirow{7}{*}{-0.8} & 20 & 0.9166 & 0.9223 & 0.9277 & 0.9325 & 0.9377 & 0.9423 & \multirow{7}{*}{-0.1} & 20 & 0.9107 & 0.9172 & 0.9236 & 0.9304 & 0.9361 & 0.9422 \\
\hline & 25 & 0.9209 & 0.9248 & 0.9293 & 0.9327 & 0.9368 & 0.9398 & & 25 & 0.9165 & 0.9229 & 0.9284 & 0.9334 & 0.9371 & 0.9424 \\
\hline & 30 & 0.929 & 0.9318 & 0.9346 & 0.9378 & 0.9405 & 0.9434 & & 30 & 0.9214 & 0.9256 & 0.9291 & 0.9343 & 0.9382 & 0.9425 \\
\hline & 35 & 0.9328 & 0.9358 & 0.939 & 0.9419 & 0.944 & 0.9469 & & 35 & 0.9289 & 0.9327 & 0.936 & 0.9389 & 0.9428 & 0.9474 \\
\hline & 40 & 0.9301 & 0.9319 & 0.9348 & 0.9377 & 0.9399 & 0.943 & & 40 & 0.9312 & 0.9345 & 0.937 & 0.9402 & 0.9432 & 0.9458 \\
\hline & 45 & 0.9335 & 0.9367 & 0.9392 & 0.9409 & 0.9426 & 0.9442 & & 45 & 0.9294 & 0.9328 & 0.9358 & 0.9379 & 0.9403 & 0.9428 \\
\hline & 50 & 0.9345 & 0.9363 & 0.9389 & 0.9409 & 0.9431 & 0.9454 & & 50 & 0.9324 & 0.9352 & 0.9377 & 0.9404 & 0.9431 & 0.9453 \\
\hline \multirow{7}{*}{-0.7} & 20 & 0.9099 & 0.9144 & 0.9207 & 0.9272 & 0.933 & 0.9384 & \multirow{7}{*}{0} & 20 & 0.909 & 0.9163 & 0.9233 & 0.9309 & 0.9376 & 0.9434 \\
\hline & 25 & 0.9222 & 0.925 & 0.929 & 0.9345 & 0.9382 & 0.9421 & & 25 & 0.9217 & 0.926 & 0.9318 & 0.9356 & 0.939 & 0.9436 \\
\hline & 30 & 0.923 & 0.9269 & 0.9302 & 0.934 & 0.9375 & 0.9412 & & 30 & 0.9231 & 0.9274 & 0.9312 & 0.9348 & 0.9394 & 0.9438 \\
\hline & 35 & 0.9322 & 0.9343 & 0.9375 & 0.9404 & 0.9434 & 0.946 & & 35 & 0.9264 & 0.9302 & 0.9339 & 0.9372 & 0.9412 & 0.9444 \\
\hline & 40 & 0.9343 & 0.9367 & 0.9393 & 0.9421 & 0.9452 & 0.9471 & & 40 & 0.9274 & 0.9301 & 0.9329 & 0.9356 & 0.9393 & 0.942 \\
\hline & 45 & 0.9323 & 0.9346 & 0.9371 & 0.939 & 0.941 & 0.944 & & 45 & 0.9339 & 0.9362 & 0.9395 & 0.9422 & 0.9446 & 0.9473 \\
\hline & 50 & 0.9343 & 0.9364 & 0.9392 & 0.9411 & 0.9427 & 0.945 & & 50 & 0.932 & 0.9345 & 0.9368 & 0.939 & 0.9417 & 0.9441 \\
\hline \multirow{7}{*}{-0.6} & 20 & 0.9115 & 0.9167 & 0.9228 & 0.929 & 0.9346 & 0.9405 & \multirow{7}{*}{0.1} & 20 & 0.9069 & 0.9132 & 0.9191 & 0.9264 & 0.9342 & 0.9414 \\
\hline & 25 & 0.918 & 0.922 & 0.9262 & 0.9326 & 0.9374 & 0.9398 & & 25 & 0.9149 & 0.9204 & 0.9258 & 0.9326 & 0.9365 & 0.9418 \\
\hline & 30 & 0.9157 & 0.9199 & 0.9241 & 0.928 & 0.9317 & 0.9348 & & 30 & 0.9237 & 0.9287 & 0.9336 & 0.9368 & 0.9408 & 0.9451 \\
\hline & 35 & 0.9259 & 0.9301 & 0.9334 & 0.9363 & 0.9393 & 0.9441 & & 35 & 0.9229 & 0.9259 & 0.9294 & 0.9324 & 0.9363 & 0.9402 \\
\hline & 40 & 0.9352 & 0.9379 & 0.9398 & 0.9422 & 0.9456 & 0.9476 & & 40 & 0.9311 & 0.9348 & 0.9373 & 0.9388 & 0.9423 & 0.9446 \\
\hline & 45 & 0.9298 & 0.9335 & 0.936 & 0.9382 & 0.9401 & 0.9428 & & 45 & 0.9302 & 0.9333 & 0.9353 & 0.9386 & 0.9421 & 0.9458 \\
\hline & 50 & 0.9347 & 0.938 & 0.9394 & 0.9419 & 0.9441 & 0.9462 & & 50 & 0.9333 & 0.9353 & 0.9375 & 0.9387 & 0.9412 & 0.9439 \\
\hline \multirow{7}{*}{-0.5} & 20 & 0.9141 & 0.921 & 0.9255 & 0.9305 & 0.9363 & 0.9425 & \multirow{7}{*}{0.2} & 20 & 0.9116 & 0.9183 & 0.9249 & 0.9305 & 0.9361 & 0.9422 \\
\hline & 25 & 0.9158 & 0.9204 & 0.9251 & 0.9296 & 0.9331 & 0.9379 & & 25 & 0.9192 & 0.9243 & 0.9293 & 0.9351 & 0.9395 & 0.9445 \\
\hline & 30 & 0.9217 & 0.9254 & 0.9297 & 0.9336 & 0.9377 & 0.9415 & & 30 & 0.9199 & 0.9252 & 0.9281 & 0.9322 & 0.937 & 0.9406 \\
\hline & 35 & 0.9305 & 0.9336 & 0.937 & 0.94 & 0.9429 & 0.9459 & & 35 & 0.9255 & 0.9282 & 0.9323 & 0.9355 & 0.9396 & 0.9428 \\
\hline & 40 & 0.9303 & 0.9334 & 0.9353 & 0.9389 & 0.9414 & 0.9436 & & 40 & 0.9307 & 0.9339 & 0.9366 & 0.9396 & 0.9425 & 0.9453 \\
\hline & 45 & 0.9334 & 0.9363 & 0.9387 & 0.9416 & 0.9441 & 0.9474 & & 45 & 0.9322 & 0.9349 & 0.9375 & 0.9414 & 0.9443 & 0.9466 \\
\hline & 50 & 0.9319 & 0.934 & 0.9364 & 0.9385 & 0.9408 & 0.9433 & & 50 & 0.9311 & 0.9341 & 0.9365 & 0.9385 & 0.9409 & 0.9436 \\
\hline & 20 & 0.9134 & 0.9201 & 0.925 & 0.9321 & 0.938 & 0.9424 & & 20 & 0.9099 & 0.9158 & 0.9221 & 0.93 & 0.9358 & 0.9425 \\
\hline & 25 & 0.9136 & 0.9183 & 0.9228 & 0.9282 & 0.9327 & 0.9375 & & 25 & 0.9229 & 0.9264 & 0.9311 & 0.9353 & 0.9411 & 0.9447 \\
\hline & 30 & 0.9251 & 0.9289 & 0.9331 & 0.9374 & 0.9422 & 0.9463 & & 30 & 0.9235 & 0.9265 & 0.9303 & 0.9343 & 0.9387 & 0.9431 \\
\hline-0.4 & 35 & 0.9293 & 0.9327 & 0.9356 & 0.9399 & 0.9436 & 0.9477 & 0.3 & 35 & 0.9304 & 0.9341 & 0.9388 & 0.9417 & 0.9444 & 0.9476 \\
\hline & 40 & 0.9265 & 0.9295 & 0.9333 & 0.9365 & 0.9397 & 0.9429 & & 40 & 0.9292 & 0.9319 & 0.9344 & 0.9379 & 0.9408 & 0.9442 \\
\hline & 45 & 0.9359 & 0.9384 & 0.9407 & 0.9437 & 0.9474 & 0.9486 & & 45 & 0.93 & 0.933 & 0.9356 & 0.9385 & 0.941 & 0.9432 \\
\hline & 50 & 0.9349 & 0.937 & 0.9386 & 0.9412 & 0.9436 & 0.946 & & 50 & 0.9367 & 0.9392 & 0.9423 & 0.9445 & 0.9457 & 0.9478 \\
\hline & 20 & 0.9105 & 0.9186 & 0.9242 & 0.9298 & 0.935 & 0.9405 & & 20 & 0.9085 & 0.9147 & 0.9206 & 0.927 & 0.9344 & 0.9412 \\
\hline & 25 & 0.9184 & 0.9244 & 0.9291 & 0.9349 & 0.939 & 0.9437 & & 25 & 0.9131 & 0.9179 & 0.9233 & 0.9286 & 0.9343 & 0.9393 \\
\hline & 30 & 0.9222 & 0.9262 & 0.9304 & 0.9339 & 0.939 & 0.9429 & & 30 & 0.9236 & 0.9282 & 0.9328 & 0.9359 & 0.94 & 0.9441 \\
\hline-0.3 & 35 & 0.9282 & 0.9311 & 0.9344 & 0.9376 & 0.9419 & 0.945 & 0.4 & 35 & 0.9297 & 0.9325 & 0.936 & 0.9388 & 0.9424 & 0.9455 \\
\hline & 40 & 0.9281 & 0.9309 & 0.9337 & 0.9372 & 0.9399 & 0.9427 & & 40 & 0.9279 & 0.9313 & 0.9343 & 0.9365 & 0.9389 & 0.9422 \\
\hline & 45 & 0.9319 & 0.9351 & 0.9374 & 0.9403 & 0.9434 & 0.9464 & & 45 & 0.9291 & 0.9317 & 0.9342 & 0.9365 & 0.9385 & 0.9411 \\
\hline & 50 & 0.9334 & 0.9363 & 0.9382 & 0.94 & 0.9425 & 0.9446 & & 50 & 0.9313 & 0.9335 & 0.9359 & 0.9382 & 0.9412 & 0.9436 \\
\hline
\end{tabular}

Table 4.4: Comparison Denominadors MLE using Confidence Interval in Expression (4.3) 


\begin{tabular}{|r|c|r|r|r|r|r|r|}
\hline & & \multicolumn{6}{|c|}{ DENOMINADOR } \\
\hline RHO & $\begin{array}{c}\text { SAMPLE } \\
\text { SIZE }\end{array}$ & $\mathbf{n}$ & $\mathbf{n}-\mathbf{1}$ & $\mathbf{n - 2}$ & $\mathbf{n}-\mathbf{3}$ & $\mathbf{n}-\mathbf{4}$ & $\mathbf{n}-\mathbf{5}$ \\
\hline & $\mathbf{2 0}$ & 0.9115 & 0.9174 & 0.9225 & 0.9298 & 0.9355 & 0.9413 \\
& $\mathbf{2 5}$ & 0.9218 & 0.928 & 0.9327 & 0.9375 & 0.9414 & 0.9448 \\
& $\mathbf{3 0}$ & 0.9243 & 0.9277 & 0.9316 & 0.9346 & 0.9379 & 0.9425 \\
$\mathbf{0 . 5}$ & $\mathbf{3 5}$ & 0.927 & 0.9304 & 0.9337 & 0.9372 & 0.941 & 0.9449 \\
& $\mathbf{4 0}$ & 0.9291 & 0.9324 & 0.935 & 0.9376 & 0.9401 & 0.9427 \\
& $\mathbf{4 5}$ & 0.9316 & 0.9335 & 0.9349 & 0.9374 & 0.9407 & 0.9435 \\
& $\mathbf{5 0}$ & 0.9333 & 0.9358 & 0.9383 & 0.9403 & 0.9422 & 0.9447 \\
\hline & $\mathbf{2 0}$ & 0.9098 & 0.9165 & 0.9218 & 0.9265 & 0.9335 & 0.9389 \\
& $\mathbf{2 5}$ & 0.9198 & 0.9253 & 0.9304 & 0.9349 & 0.9393 & 0.943 \\
& $\mathbf{3 0}$ & 0.9249 & 0.9279 & 0.9316 & 0.9361 & 0.9396 & 0.9429 \\
$\mathbf{0 . 6}$ & $\mathbf{3 5}$ & 0.9271 & 0.9303 & 0.9333 & 0.9377 & 0.9409 & 0.9439 \\
& $\mathbf{4 0}$ & 0.9339 & 0.9366 & 0.9392 & 0.9425 & 0.9447 & 0.9462 \\
& $\mathbf{4 5}$ & 0.9334 & 0.9361 & 0.9379 & 0.9406 & 0.9434 & 0.9455 \\
& $\mathbf{5 0}$ & 0.9374 & 0.9391 & 0.9416 & 0.9444 & 0.946 & 0.9475 \\
\hline & $\mathbf{2 0}$ & 0.9155 & 0.9214 & 0.9277 & 0.9327 & 0.9384 & 0.9437 \\
& $\mathbf{2 5}$ & 0.921 & 0.9254 & 0.9287 & 0.9329 & 0.9365 & 0.9402 \\
& $\mathbf{3 0}$ & 0.9265 & 0.9316 & 0.9358 & 0.9391 & 0.9424 & 0.946 \\
$\mathbf{0 . 7}$ & $\mathbf{3 5}$ & 0.9303 & 0.9329 & 0.9359 & 0.9374 & 0.9402 & 0.9431 \\
& $\mathbf{4 0}$ & 0.9289 & 0.9305 & 0.9341 & 0.9373 & 0.9406 & 0.9428 \\
& $\mathbf{4 5}$ & 0.9339 & 0.9357 & 0.9378 & 0.9405 & 0.9425 & 0.9448 \\
& $\mathbf{5 0}$ & 0.9318 & 0.9342 & 0.9363 & 0.9383 & 0.9411 & 0.9434 \\
& & & & & & & \\
& & & & &
\end{tabular}

\begin{tabular}{|c|c|c|r|r|r|c|c|}
\hline & & \multicolumn{6}{|c|}{ DENOMINADOR } \\
\hline RHO & $\begin{array}{c}\text { SAMPLE } \\
\text { SIZE }\end{array}$ & $\mathbf{n}$ & $\mathbf{n}-\mathbf{1}$ & \multicolumn{1}{|c|}{$\mathbf{n - 2}$} & $\mathbf{n}-\mathbf{3}$ & $\mathbf{n}-\mathbf{4}$ & $\mathbf{n}-\mathbf{5}$ \\
\hline \multirow{6}{*}{$\mathbf{0 . 8}$} & $\mathbf{2 0}$ & 0.9094 & 0.9153 & 0.9211 & 0.9263 & 0.9321 & 0.9376 \\
& $\mathbf{2 5}$ & 0.9131 & 0.9175 & 0.9227 & 0.927 & 0.9318 & 0.9357 \\
& $\mathbf{3 0}$ & 0.9254 & 0.928 & 0.932 & 0.9345 & 0.9383 & 0.9409 \\
& $\mathbf{3 5}$ & 0.9276 & 0.9311 & 0.9335 & 0.9355 & 0.9382 & 0.9413 \\
& $\mathbf{4 0}$ & 0.9317 & 0.9341 & 0.9364 & 0.9385 & 0.9412 & 0.9435 \\
& $\mathbf{4 5}$ & 0.9376 & 0.9399 & 0.9414 & 0.9438 & 0.9459 & 0.9476 \\
& $\mathbf{5 0}$ & 0.9366 & 0.9384 & 0.9403 & 0.9422 & 0.9445 & 0.9471 \\
\hline & $\mathbf{2 0}$ & 0.911 & 0.915 & 0.9213 & 0.9259 & 0.9303 & 0.9357 \\
& $\mathbf{2 5}$ & 0.9199 & 0.9232 & 0.9265 & 0.9305 & 0.9337 & 0.9373 \\
& $\mathbf{3 0}$ & 0.9224 & 0.9257 & 0.9294 & 0.9322 & 0.9358 & 0.9394 \\
$\mathbf{0 . 9}$ & $\mathbf{3 5}$ & 0.926 & 0.9288 & 0.9314 & 0.9346 & 0.9379 & 0.9402 \\
& $\mathbf{4 0}$ & 0.9283 & 0.9308 & 0.9338 & 0.9366 & 0.9389 & 0.9417 \\
& $\mathbf{4 5}$ & 0.9375 & 0.9391 & 0.9411 & 0.9432 & 0.9449 & 0.9467 \\
& $\mathbf{5 0}$ & 0.9323 & 0.9348 & 0.937 & 0.9387 & 0.9414 & 0.9439 \\
\hline
\end{tabular}

Table 4.5: Cont. Comparison Denominadors MLE using Confidence Interval in Expression. (4.3) 

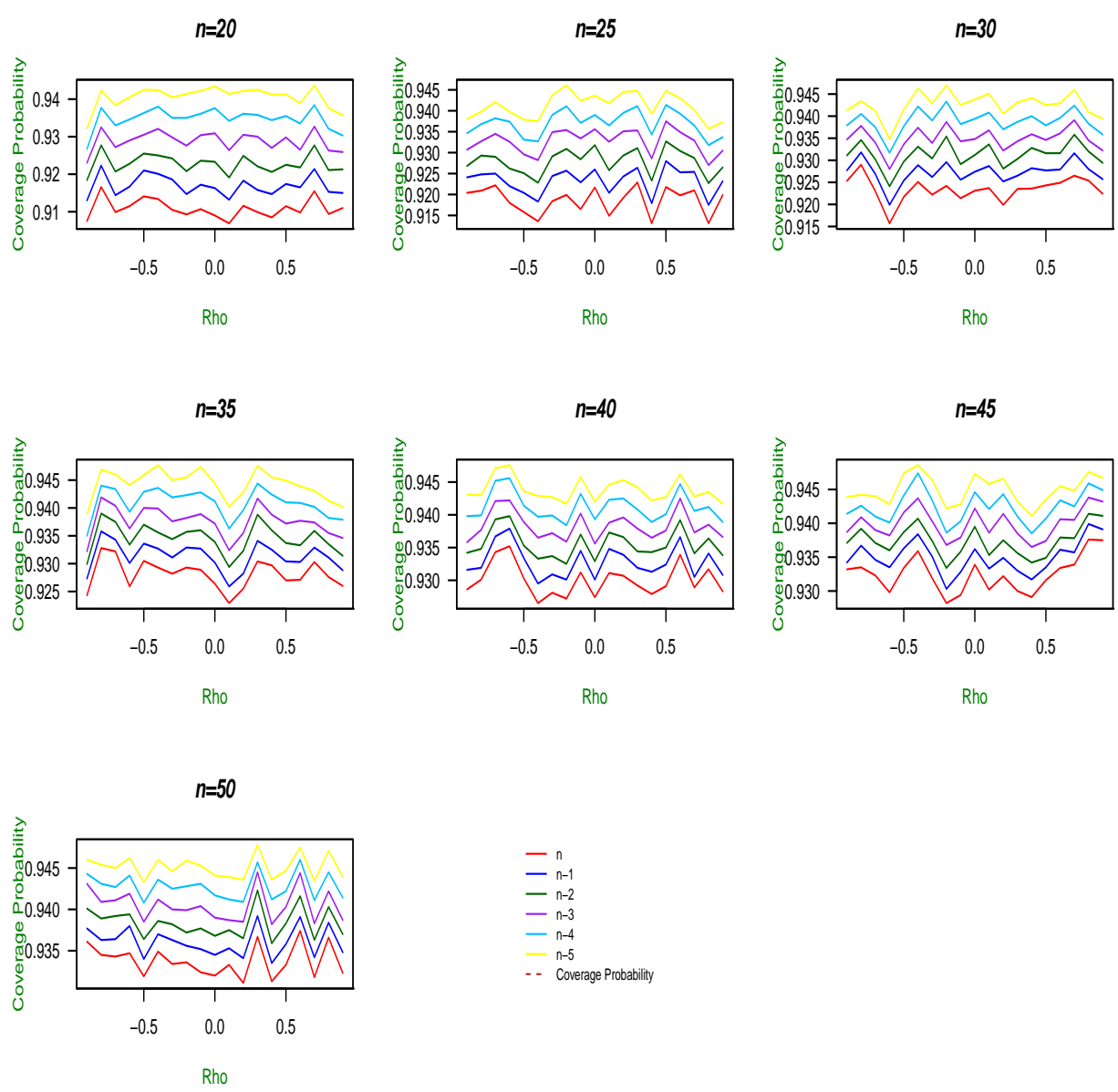

Figure 4.5: Comparison Denominators for MLE using Confidence Interval in Expression. (4.3)

It can be observed from Figure 4.5 that the $95 \%$ level for the confidence interval for $\rho$ is not attained for the values of $k$ and all sample sizes. 


\begin{tabular}{|c|c|c|c|c|c|c|c|}
\hline \multirow{2}{*}{\begin{tabular}{|l} 
\\
RHO
\end{tabular}} & \multirow[b]{2}{*}{$\begin{array}{c}\text { SAMPLE } \\
\text { SIZE }\end{array}$} & \multicolumn{6}{|c|}{ DENOMINADOR } \\
\hline & & $\mathbf{n}$ & $n-1$ & $n-2$ & $n-3$ & $n-4$ & $n-5$ \\
\hline \multirow{7}{*}{-0.9} & 20 & 0.8832 & 0.8883 & 0.8938 & 0.8998 & 0.9066 & 0.9131 \\
\hline & 25 & 0.9039 & 0.9075 & 0.9112 & 0.9149 & 0.9191 & 0.9227 \\
\hline & 30 & 0.9084 & 0.9112 & 0.9141 & 0.9175 & 0.9222 & 0.9266 \\
\hline & 35 & 0.9117 & 0.9146 & 0.9177 & 0.9204 & 0.9234 & 0.9273 \\
\hline & 40 & 0.9169 & 0.9191 & 0.9219 & 0.9243 & 0.9267 & 0.9293 \\
\hline & 45 & 0.9245 & 0.9269 & 0.9289 & 0.9310 & 0.9331 & 0.9347 \\
\hline & 50 & 0.9270 & 0.9291 & 0.9309 & 0.9338 & 0.9351 & 0.9373 \\
\hline \multirow{7}{*}{-0.8} & 20 & 0.8956 & 0.9021 & 0.9078 & 0.9141 & 0.9200 & 0.9256 \\
\hline & 25 & 0.9063 & 0.9096 & 0.9141 & 0.9192 & 0.9246 & 0.9283 \\
\hline & 30 & 0.9167 & 0.9199 & 0.9233 & 0.9265 & 0.9296 & 0.9331 \\
\hline & 35 & 0.9214 & 0.9244 & 0.9269 & 0.9301 & 0.9328 & 0.9366 \\
\hline & 40 & 0.9201 & 0.9220 & 0.9259 & 0.9292 & 0.9318 & 0.9341 \\
\hline & 45 & 0.9243 & 0.9269 & 0.9291 & 0.9309 & 0.9330 & 0.9351 \\
\hline & 50 & 0.9268 & 0.9286 & 0.9312 & 0.9334 & 0.9355 & 0.9379 \\
\hline \multirow{7}{*}{-0.7} & 20 & 0.8929 & 0.8976 & 0.9048 & 0.9107 & 0.9166 & 0.9223 \\
\hline & 25 & 0.9078 & 0.9126 & 0.9185 & 0.9234 & 0.9272 & 0.9309 \\
\hline & 30 & 0.9144 & 0.9176 & 0.9217 & 0.9254 & 0.9283 & 0.9324 \\
\hline & 35 & 0.9228 & 0.9265 & 0.9290 & 0.9316 & 0.9346 & 0.9382 \\
\hline & 40 & 0.9272 & 0.9295 & 0.9322 & 0.9344 & 0.9370 & 0.9399 \\
\hline & 45 & 0.9259 & 0.9285 & 0.9305 & 0.9327 & 0.9358 & 0.9380 \\
\hline & 50 & 0.9290 & 0.9311 & 0.9340 & 0.9362 & 0.9383 & 0.9397 \\
\hline \multirow{7}{*}{-0.6} & 20 & 0.8977 & 0.9042 & 0.9115 & 0.9177 & 0.9234 & 0.9287 \\
\hline & 25 & 0.9072 & 0.9109 & 0.9163 & 0.9224 & 0.9268 & 0.9319 \\
\hline & 30 & 0.9054 & 0.9099 & 0.9145 & 0.9181 & 0.9221 & 0.9255 \\
\hline & 35 & 0.9191 & 0.9220 & 0.9251 & 0.9282 & 0.9304 & 0.9351 \\
\hline & 40 & 0.9286 & 0.9307 & 0.9339 & 0.9361 & 0.9397 & 0.9420 \\
\hline & 45 & 0.9254 & 0.9283 & 0.9307 & 0.9339 & 0.9355 & 0.9383 \\
\hline & 50 & 0.9284 & 0.9307 & 0.9322 & 0.9347 & 0.9369 & 0.9393 \\
\hline \multirow{7}{*}{-0.5} & 20 & 0.9025 & 0.9086 & \begin{tabular}{|c|}
0.9138 \\
\end{tabular} & 0.9196 & 0.9250 & 0.9310 \\
\hline & 25 & 0.9058 & 0.9111 & 0.9148 & 0.9201 & 0.9243 & 0.9287 \\
\hline & 30 & 0.9124 & 0.9172 & 0.9220 & 0.9255 & 0.9298 & 0.9323 \\
\hline & 35 & 0.9245 & 0.9281 & 0.9317 & 0.9346 & 0.9368 & 0.9400 \\
\hline & 40 & 0.9241 & 0.9274 & 0.9308 & 0.9338 & 0.9365 & 0.9389 \\
\hline & 45 & 0.9283 & 0.9311 & 0.9340 & 0.9365 & 0.9394 & 0.9422 \\
\hline & 50 & 0.9276 & 0.9299 & 0.9319 & 0.9336 & 0.9368 & 0.9400 \\
\hline \multirow{7}{*}{-0.4} & 20 & 0.9021 & 0.9080 & 0.9141 & 0.9203 & 0.9268 & 0.9339 \\
\hline & 25 & 0.9044 & 0.9102 & 0.9153 & 0.9197 & 0.9256 & 0.9305 \\
\hline & 30 & 0.9161 & 0.9199 & 0.9240 & 0.9294 & 0.9334 & 0.9374 \\
\hline & 35 & 0.9247 & 0.9281 & 0.9309 & 0.9354 & 0.9387 & 0.9417 \\
\hline & 40 & 0.9213 & 0.9241 & 0.9282 & 0.9316 & 0.9350 & 0.9380 \\
\hline & 45 & 0.9320 & 0.9344 & 0.9365 & 0.9396 & 0.9435 & 0.9457 \\
\hline & 50 & 0.9309 & 0.9331 & 0.9350 & 0.9374 & 0.9401 & 0.9429 \\
\hline \multirow{7}{*}{-0.3} & 20 & 0.8995 & 0.9077 & 0.9143 & 0.9207 & 0.9268 & 0.9321 \\
\hline & 25 & 0.9099 & 0.9155 & 0.9211 & 0.9267 & 0.9313 & 0.9362 \\
\hline & 30 & 0.9177 & 0.9224 & 0.9253 & 0.9289 & 0.9334 & 0.9372 \\
\hline & 35 & 0.9229 & 0.9262 & 0.9289 & 0.9327 & 0.9364 & 0.9395 \\
\hline & 40 & 0.9240 & 0.9270 & 0.9294 & 0.9324 & 0.9351 & 0.9384 \\
\hline & 45 & 0.9279 & 0.9308 & 0.9333 & 0.9358 & 0.9383 & 0.9419 \\
\hline & 50 & 0.9306 & 0.9337 & 0.9352 & 0.9373 & 0.9399 & 0.9427 \\
\hline
\end{tabular}

\begin{tabular}{|c|c|c|c|c|c|c|c|}
\hline \multirow[b]{2}{*}{ RHO } & \multirow[b]{2}{*}{$\begin{array}{c}\text { SAMPLE } \\
\text { SIZE }\end{array}$} & \multicolumn{6}{|c|}{ DENOMINADOR } \\
\hline & & $\mathbf{n}$ & $n-1$ & $n-2$ & $n-3$ & $n-4$ & $n-5$ \\
\hline \multirow{7}{*}{-0.2} & 20 & 0.9002 & 0.9054 & 0.9120 & 0.9193 & 0.9269 & 0.9332 \\
\hline & 25 & 0.9130 & 0.9187 & 0.9237 & 0.9293 & 0.9357 & 0.9399 \\
\hline & 30 & 0.9173 & 0.9225 & 0.9287 & 0.9328 & 0.9379 & 0.9419 \\
\hline & 35 & 0.9232 & 0.9281 & 0.9312 & 0.9347 & 0.9378 & 0.9416 \\
\hline & 40 & 0.9220 & 0.9256 & 0.9289 & 0.9318 & 0.9351 & 0.9381 \\
\hline & 45 & 0.9240 & 0.9266 & 0.9293 & 0.9325 & 0.9354 & 0.9395 \\
\hline & 50 & 0.9298 & 0.9328 & 0.9342 & 0.9366 & 0.9399 & 0.9430 \\
\hline \multirow{7}{*}{-0.1} & 20 & 0.9007 & 0.9079 & 0.9149 & 0.9223 & 0.9282 & 0.9355 \\
\hline & 25 & 0.9089 & 0.9150 & 0.9216 & 0.9264 & 0.9319 & 0.9366 \\
\hline & 30 & 0.9169 & 0.9207 & 0.9239 & 0.9272 & 0.9328 & 0.9373 \\
\hline & 35 & 0.9242 & 0.9281 & 0.9323 & 0.9351 & 0.9387 & 0.9420 \\
\hline & 40 & 0.9270 & 0.9301 & 0.9329 & 0.9367 & 0.9392 & 0.9423 \\
\hline & 45 & 0.9264 & 0.9291 & 0.9319 & 0.9352 & 0.9378 & 0.9404 \\
\hline & 50 & 0.9296 & 0.9323 & 0.9344 & 0.9378 & 0.9404 & 0.9433 \\
\hline \multirow{7}{*}{$\mathbf{0}$} & 20 & 0.8993 & 0.9073 & 0.9148 & 0.9225 & 0.9300 & 0.9369 \\
\hline & 25 & 0.9157 & 0.9206 & 0.9257 & 0.9306 & 0.9345 & 0.9391 \\
\hline & 30 & 0.9175 & 0.9218 & 0.9256 & 0.9294 & 0.9344 & 0.9388 \\
\hline & 35 & 0.9223 & 0.9263 & 0.9299 & 0.9330 & 0.9371 & 0.9406 \\
\hline & 40 & 0.9240 & 0.9271 & 0.9299 & 0.9323 & 0.9356 & 0.9390 \\
\hline & 45 & 0.9289 & 0.9321 & 0.9361 & 0.9388 & 0.9418 & 0.9448 \\
\hline & 50 & 0.9293 & 0.9320 & 0.9344 & 0.9373 & 0.9400 & 0.9424 \\
\hline \multirow{7}{*}{0.1} & 20 & 0.8983 & 0.9050 & 0.9104 & 0.9173 & 0.9245 & 0.9322 \\
\hline & 25 & 0.9085 & 0.9129 & 0.9197 & 0.9259 & 0.9307 & 0.9352 \\
\hline & 30 & 0.9189 & 0.9234 & 0.9280 & 0.9317 & 0.9362 & 0.9398 \\
\hline & 35 & 0.9183 & 0.9219 & 0.9251 & 0.9289 & 0.9328 & 0.9364 \\
\hline & 40 & 0.9279 & 0.9314 & 0.9348 & 0.9372 & 0.9397 & 0.9415 \\
\hline & 45 & 0.9273 & 0.9300 & 0.9330 & 0.9360 & 0.9390 & 0.9429 \\
\hline & 50 & 0.9294 & 0.9317 & 0.9344 & 0.9364 & 0.9385 & 0.9412 \\
\hline \multirow{7}{*}{0.2} & 20 & 0.9005 & 0.9082 & 0.9148 & 0.9209 & 0.9279 & 0.9341 \\
\hline & 25 & 0.9125 & 0.9173 & 0.9222 & 0.9282 & 0.9340 & 0.9389 \\
\hline & 30 & 0.9152 & 0.9202 & 0.9233 & 0.9280 & 0.9330 & 0.9362 \\
\hline & 35 & 0.9216 & 0.9247 & 0.9285 & 0.9319 & 0.9347 & 0.9383 \\
\hline & 40 & 0.9272 & 0.9307 & 0.9333 & 0.9357 & 0.9390 & 0.9420 \\
\hline & 45 & 0.9282 & 0.9318 & 0.9341 & 0.9373 & 0.9400 & 0.9436 \\
\hline & 50 & 0.9282 & 0.9309 & 0.9337 & 0.9365 & 0.9385 & 0.9410 \\
\hline \multirow{7}{*}{0.3} & 20 & 0.8993 & 0.9044 & 0.9122 & 0.9192 & 0.9259 & 0.9327 \\
\hline & 25 & 0.9145 & 0.9183 & 0.9235 & 0.9291 & 0.9350 & 0.9387 \\
\hline & 30 & 0.9165 & 0.9204 & 0.9243 & 0.9287 & 0.9321 & 0.9368 \\
\hline & 35 & 0.9255 & 0.9278 & 0.9323 & 0.9353 & 0.9388 & 0.9432 \\
\hline & 40 & 0.9249 & 0.9276 & 0.9305 & 0.9343 & 0.9374 & 0.9406 \\
\hline & 45 & 0.9257 & 0.9284 & 0.9308 & 0.9336 & 0.9365 & 0.9397 \\
\hline & 50 & 0.9324 & 0.9354 & 0.9393 & 0.9413 & 0.9430 & 0.9449 \\
\hline \multirow{7}{*}{0.4} & 20 & 0.8962 & 0.9020 & 0.9093 & 0.9168 & 0.9238 & 0.9319 \\
\hline & 25 & 0.9028 & 0.9085 & 0.9137 & 0.9193 & 0.9258 & 0.9312 \\
\hline & 30 & 0.9171 & 0.9225 & 0.9269 & 0.9303 & 0.9338 & 0.9370 \\
\hline & 35 & 0.9221 & 0.9255 & 0.9291 & 0.9325 & 0.9354 & 0.9390 \\
\hline & 40 & 0.9227 & 0.9256 & 0.9286 & 0.9312 & 0.9338 & 0.9364 \\
\hline & 45 & 0.9248 & 0.9272 & 0.9298 & 0.9328 & 0.9349 & 0.9373 \\
\hline & 50 & 0.9281 & 0.9301 & 0.9324 & 0.9346 & 0.9372 & 0.9404 \\
\hline
\end{tabular}

Table 4.6: Comparison Denominadors Pearson using Confidence Interval in Expression. (4.3) 


\begin{tabular}{|r|c|r|r|r|r|r|r|}
\hline & & \multicolumn{5}{|c|}{ DENOMINADOR } \\
\hline \multirow{2}{*}{ RHO } & $\begin{array}{c}\text { SAMPLE } \\
\text { SIZE }\end{array}$ & $\mathbf{n}$ & $\mathbf{n}-\mathbf{1}$ & $\mathbf{n}-\mathbf{2}$ & $\mathbf{n}-\mathbf{3}$ & $\mathbf{n}-\mathbf{4}$ & $\mathbf{n - 5}$ \\
\hline \multirow{5}{*}{$\mathbf{0 . 5}$} & $\mathbf{2 0}$ & 0.8987 & 0.9048 & 0.9102 & 0.9179 & 0.9243 & 0.9301 \\
& $\mathbf{2 5}$ & 0.9127 & 0.9179 & 0.9231 & 0.9284 & 0.9328 & 0.9375 \\
& $\mathbf{3 0}$ & 0.916 & 0.9201 & 0.9248 & 0.9274 & 0.931 & 0.9352 \\
& $\mathbf{3 5}$ & 0.9186 & 0.923 & 0.9266 & 0.9295 & 0.9337 & 0.9376 \\
& $\mathbf{4 0}$ & 0.922 & 0.9263 & 0.9285 & 0.9308 & 0.9332 & 0.9367 \\
& $\mathbf{4 5}$ & 0.9265 & 0.9285 & 0.9312 & 0.9331 & 0.9361 & 0.9389 \\
& $\mathbf{5 0}$ & 0.9285 & 0.9314 & 0.9337 & 0.9358 & 0.9374 & 0.9399 \\
\hline \multirow{5}{*}{$\mathbf{0 . 6}$} & $\mathbf{2 0}$ & 0.8931 & 0.8987 & 0.9062 & 0.9119 & 0.9197 & 0.9258 \\
& $\mathbf{2 5}$ & 0.9063 & 0.9118 & 0.9184 & 0.9239 & 0.9296 & 0.9349 \\
& $\mathbf{3 0}$ & 0.9141 & 0.9178 & 0.9218 & 0.9266 & 0.9307 & 0.9355 \\
& $\mathbf{3 5}$ & 0.9199 & 0.9228 & 0.926 & 0.93 & 0.9332 & 0.9368 \\
& $\mathbf{4 0}$ & 0.9262 & 0.9285 & 0.9318 & 0.9351 & 0.938 & 0.9413 \\
& $\mathbf{4 5}$ & 0.9291 & 0.931 & 0.9335 & 0.9354 & 0.9384 & 0.9407 \\
& $\mathbf{5 0}$ & 0.9309 & 0.9338 & 0.9365 & 0.9392 & 0.9412 & 0.9425 \\
\hline \multirow{6}{*}{$\mathbf{0 . 7}$} & $\mathbf{2 0}$ & 0.8997 & 0.9048 & 0.912 & 0.9174 & 0.9227 & 0.9296 \\
& $\mathbf{2 5}$ & 0.9096 & 0.914 & 0.9168 & 0.9208 & 0.9259 & 0.9299 \\
& $\mathbf{3 0}$ & 0.9164 & 0.9206 & 0.9243 & 0.9284 & 0.9319 & 0.9358 \\
& $\mathbf{4 0}$ & 0.921 & 0.9239 & 0.9262 & 0.9296 & 0.9327 & 0.9352 \\
& $\mathbf{4 5}$ & 0.9202 & 0.923 & 0.9256 & 0.9282 & 0.9319 & 0.9337 \\
& $\mathbf{5 0}$ & 0.9244 & 0.9269 & 0.9296 & 0.9322 & 0.935 & 0.9366 \\
\hline
\end{tabular}

\begin{tabular}{|r|r|r|r|r|r|r|r|}
\hline & & \multicolumn{5}{|c|}{ DENOMINADOR } \\
\hline \multirow{2}{*}{ RHO } & $\begin{array}{c}\text { SAMPLE } \\
\text { SIZE }\end{array}$ & $\mathbf{n}$ & $\mathbf{n}-\mathbf{1}$ & $\mathbf{n}-\mathbf{2}$ & $\mathbf{n}-\mathbf{3}$ & $\mathbf{n}-\mathbf{4}$ & $\mathbf{n}-\mathbf{5}$ \\
\hline & $\mathbf{2 0}$ & 0.8893 & 0.8964 & 0.9027 & 0.9093 & 0.9145 & 0.9212 \\
& $\mathbf{2 5}$ & 0.8977 & 0.9022 & 0.908 & 0.9129 & 0.9187 & 0.9238 \\
& $\mathbf{3 0}$ & 0.9143 & 0.9166 & 0.9205 & 0.9243 & 0.9276 & 0.931 \\
$\mathbf{0 . 8}$ & $\mathbf{3 5}$ & 0.9185 & 0.9212 & 0.9242 & 0.9265 & 0.9292 & 0.9324 \\
& $\mathbf{4 0}$ & 0.9233 & 0.9251 & 0.9283 & 0.9302 & 0.9324 & 0.9351 \\
& $\mathbf{4 5}$ & 0.9289 & 0.932 & 0.9345 & 0.9363 & 0.9379 & 0.9396 \\
& $\mathbf{5 0}$ & 0.9299 & 0.9318 & 0.9332 & 0.9352 & 0.937 & 0.9394 \\
\hline & $\mathbf{2 0}$ & 0.8881 & 0.8927 & 0.8978 & 0.9031 & 0.9088 & 0.9163 \\
& $\mathbf{2 5}$ & 0.901 & 0.905 & 0.9095 & 0.914 & 0.9181 & 0.9215 \\
& $\mathbf{3 0}$ & 0.9091 & 0.9119 & 0.9152 & 0.9179 & 0.9217 & 0.9262 \\
$\mathbf{0 . 9}$ & $\mathbf{3 5}$ & 0.9128 & 0.9167 & 0.9201 & 0.9222 & 0.9251 & 0.9282 \\
& $\mathbf{4 0}$ & 0.9177 & 0.9202 & 0.923 & 0.9257 & 0.9286 & 0.9318 \\
& $\mathbf{4 5}$ & 0.9264 & 0.9294 & 0.9315 & 0.9344 & 0.9364 & 0.9393 \\
& $\mathbf{5 0}$ & 0.925 & 0.9277 & 0.9297 & 0.9322 & 0.9346 & 0.9366 \\
\hline
\end{tabular}

Table 4.7: Cont. Comparison Denominadors Pearson using Confidence Interval in Expression. (4.3)

Similarly, Figure 4.6 shows us that the $95 \%$ level for the confidence interval for $\rho$ using the Pearson Estimator is not attained for neither of the values of $k$ in all sample sizes considered.

For the previous observations, since the desired level is not achieved, this distribution for $\hat{\rho}$ in Expression (4.3) will not be considered further. 

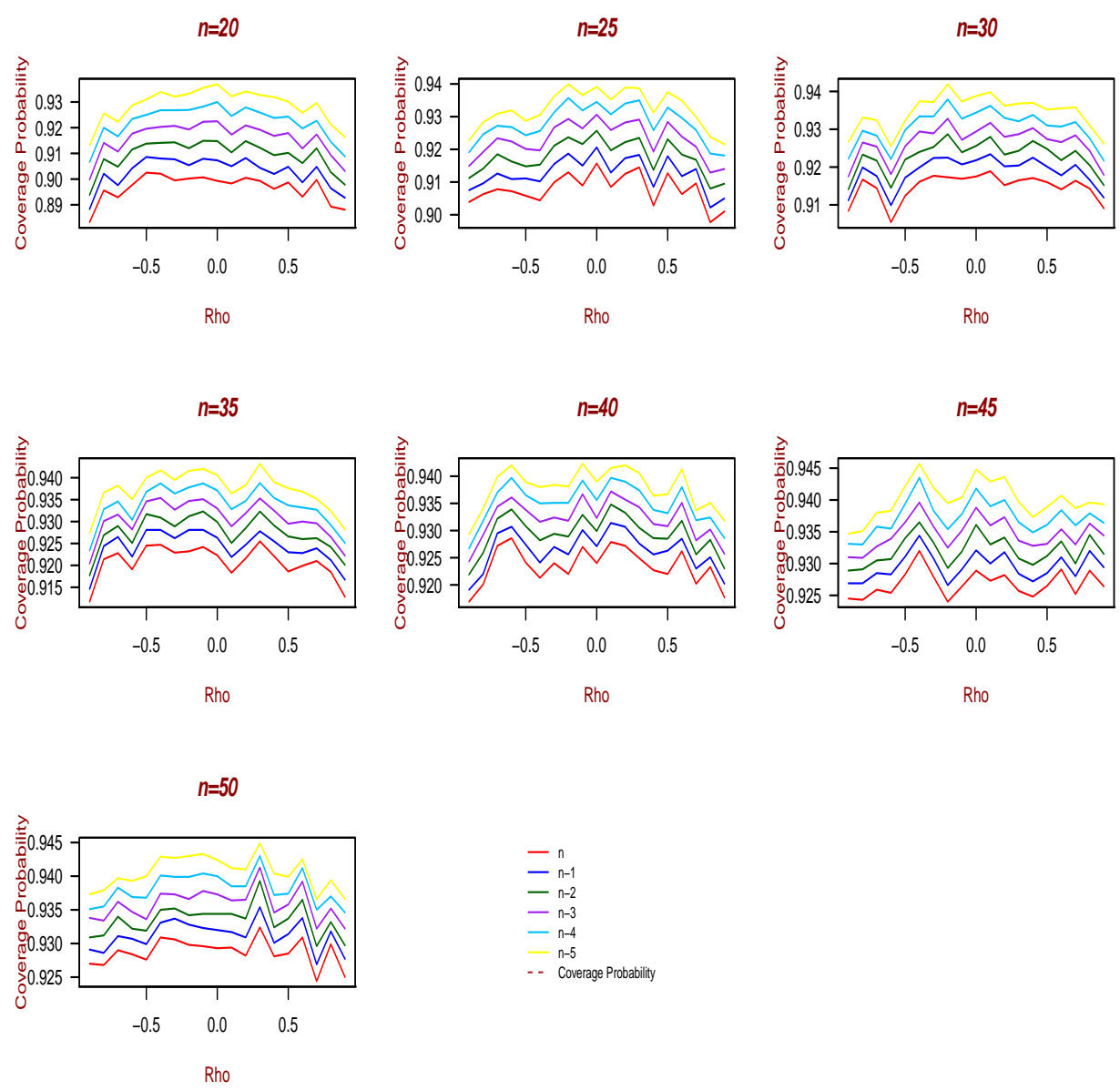

Figure 4.6: Comparison Denominators for Pearson Estimator using Confidence Interval in Espression. (4.3)

Likewise, a coverage probability is measured using the $95 \%$ confidence interval given in the expression (4.2):

$$
\left(\frac{e^{2 l}-1}{e^{2 l}+1}, \frac{e^{2 u}-1}{e^{2 u}+1}\right)
$$

where $l=\frac{1}{2} \ln \left(\frac{1+\hat{\rho}_{n}}{1-\hat{\rho}_{n}}\right)-1.96 \frac{1}{\sqrt{n-k}}$ and $u=\frac{1}{2} \ln \left(\frac{1+\hat{\rho}_{n}}{1-\hat{\rho}_{n}}\right)+1.96 \frac{1}{\sqrt{n-k}}$ for $k=0, \ldots, 5$ using both estimators. 


\begin{tabular}{|c|c|c|c|c|c|c|c|}
\hline \multirow{2}{*}{\begin{tabular}{|l} 
\\
RHO
\end{tabular}} & \multirow[b]{2}{*}{$\begin{array}{c}\text { SAMPLE } \\
\text { SIZE }\end{array}$} & \multicolumn{6}{|c|}{ DENOMINADOR } \\
\hline & & $\mathbf{n}$ & $n-1$ & $n-2$ & $n-3$ & $n-4$ & $n-5$ \\
\hline \multirow{7}{*}{-0.9} & 20 & 0.9379 & 0.9448 & 0.9517 & 0.9580 & 0.9642 & 0.9688 \\
\hline & 25 & 0.9392 & 0.9443 & 0.9500 & 0.9556 & 0.9601 & 0.9659 \\
\hline & 30 & 0.9406 & 0.9445 & 0.9488 & 0.9529 & 0.9567 & 0.9621 \\
\hline & 35 & 0.9406 & 0.9438 & 0.9481 & 0.9521 & 0.9551 & 0.9586 \\
\hline & 40 & 0.9424 & 0.9453 & 0.9478 & 0.9505 & 0.9540 & 0.9568 \\
\hline & 45 & 0.9431 & 0.9448 & 0.9483 & 0.9511 & 0.9538 & 0.9562 \\
\hline & 50 & 0.9448 & 0.9478 & 0.9506 & 0.9523 & 0.9546 & 0.9570 \\
\hline \multirow{7}{*}{-0.8} & 20 & 0.9424 & 0.9469 & 0.9537 & 0.9606 & 0.9662 & 0.9719 \\
\hline & 25 & 0.9406 & 0.9458 & 0.9507 & 0.9552 & 0.9602 & 0.9644 \\
\hline & 30 & 0.9423 & 0.9455 & 0.9505 & 0.9549 & 0.9589 & 0.9624 \\
\hline & 35 & 0.9448 & 0.9481 & 0.9512 & 0.9542 & 0.9586 & 0.9614 \\
\hline & 40 & 0.9430 & 0.9461 & 0.9489 & 0.9533 & 0.9545 & 0.9572 \\
\hline & 45 & 0.9436 & 0.9466 & 0.9491 & 0.9516 & 0.9539 & 0.9567 \\
\hline & 50 & 0.9446 & 0.9463 & 0.9485 & 0.9511 & 0.9537 & 0.9563 \\
\hline \multirow{7}{*}{-0.7} & 20 & 0.9331 & 0.9400 & 0.9470 & 0.9518 & 0.9593 & 0.9648 \\
\hline & 25 & 0.9417 & 0.9462 & 0.9504 & 0.9568 & 0.9615 & 0.9653 \\
\hline & 30 & 0.9440 & 0.9479 & 0.9514 & 0.9555 & 0.9593 & 0.9631 \\
\hline & 35 & 0.9463 & 0.9500 & 0.9526 & 0.9565 & 0.9598 & 0.9633 \\
\hline & 40 & 0.9451 & 0.9476 & 0.9502 & 0.9531 & 0.9563 & 0.9597 \\
\hline & 45 & 0.9391 & 0.9414 & 0.9440 & 0.9482 & 0.9502 & 0.9535 \\
\hline & 50 & 0.9419 & 0.9449 & 0.9470 & 0.9505 & 0.9529 & 0.9556 \\
\hline \multirow{7}{*}{-0.6} & 20 & 0.9393 & 0.9446 & 0.9501 & 0.9545 & 0.9601 & 0.9664 \\
\hline & 25 & 0.9380 & 0.9434 & 0.9488 & 0.9547 & 0.9596 & 0.9652 \\
\hline & 30 & 0.9332 & 0.9387 & 0.9428 & 0.9478 & 0.9522 & 0.9557 \\
\hline & 35 & 0.9406 & 0.9437 & 0.9472 & 0.9507 & 0.9540 & 0.9580 \\
\hline & 40 & 0.9459 & 0.9482 & 0.9510 & 0.9540 & 0.9567 & 0.9602 \\
\hline & 45 & 0.9409 & 0.9436 & 0.9458 & 0.9482 & 0.9516 & 0.9537 \\
\hline & 50 & 0.9469 & 0.9494 & 0.9519 & 0.9540 & 0.9563 & 0.9578 \\
\hline \multirow{7}{*}{-0.5} & 20 & 0.9390 & 0.9450 & 0.9502 & 0.9564 & 0.9622 & 0.9678 \\
\hline & 25 & 0.9379 & 0.9436 & 0.9492 & 0.9539 & 0.9575 & 0.9627 \\
\hline & 30 & 0.9410 & 0.9453 & 0.9497 & 0.9527 & 0.9565 & 0.9596 \\
\hline & 35 & 0.9462 & 0.9496 & 0.9533 & 0.9568 & 0.9605 & 0.9630 \\
\hline & 40 & 0.9435 & 0.9471 & 0.9507 & 0.9537 & 0.9573 & 0.9600 \\
\hline & 45 & 0.9487 & 0.9515 & 0.9546 & 0.9564 & 0.9588 & 0.9605 \\
\hline & 50 & 0.9400 & 0.9425 & 0.9445 & 0.9466 & 0.9487 & 0.9514 \\
\hline \multirow{7}{*}{-0.4} & 20 & 0.9397 & 0.9463 & 0.9519 & 0.9582 & 0.9629 & 0.9691 \\
\hline & 25 & 0.9343 & 0.9407 & 0.9453 & 0.9505 & 0.9559 & 0.9603 \\
\hline & 30 & 0.9436 & 0.9472 & 0.9514 & 0.9546 & 0.9586 & 0.9628 \\
\hline & 35 & 0.9455 & 0.9496 & 0.9519 & 0.9546 & 0.9573 & 0.9614 \\
\hline & 40 & 0.9396 & 0.9422 & 0.9440 & 0.9477 & 0.9502 & 0.9545 \\
\hline & 45 & 0.9477 & 0.9504 & 0.9523 & 0.9544 & 0.9574 & 0.9595 \\
\hline & 50 & 0.9456 & 0.9476 & 0.9501 & 0.9526 & 0.9554 & 0.9581 \\
\hline \multirow{7}{*}{-0.3} & 20 & 0.9391 & 0.9463 & 0.9513 & 0.9560 & 0.9611 & 0.9659 \\
\hline & 25 & 0.9404 & 0.9458 & 0.9514 & 0.9551 & 0.9598 & 0.9639 \\
\hline & 30 & 0.9418 & 0.9469 & 0.9503 & 0.9538 & 0.9581 & 0.9627 \\
\hline & 35 & 0.9447 & 0.9484 & 0.9513 & 0.9542 & 0.9569 & 0.9604 \\
\hline & 40 & 0.9431 & 0.9469 & 0.9498 & 0.9522 & 0.9550 & 0.9587 \\
\hline & 45 & 0.9446 & 0.9470 & 0.9488 & 0.9515 & 0.9542 & 0.9569 \\
\hline & 50 & 0.9456 & 0.9476 & 0.9505 & 0.9533 & 0.9551 & 0.9568 \\
\hline
\end{tabular}

\begin{tabular}{|c|c|c|c|c|c|c|c|}
\hline \multirow[b]{2}{*}{ RHO } & \multirow[b]{2}{*}{$\begin{array}{c}\text { SAMPLE } \\
\text { SIZE }\end{array}$} & \multicolumn{6}{|c|}{ DENOMINADOR } \\
\hline & & $\mathbf{n}$ & $n-1$ & $\mathrm{n}-2$ & $n-3$ & $n-4$ & $n-5$ \\
\hline \multirow{7}{*}{-0.2} & 20 & 0.9372 & \begin{tabular}{|l|}
0.9439 \\
\end{tabular} & 0.9498 & 0.9563 & 0.9623 & 0.9678 \\
\hline & 25 & 0.9436 & 0.9480 & 0.9529 & 0.9578 & 0.9611 & 0.9662 \\
\hline & 30 & 0.9439 & 0.9495 & 0.9520 & 0.9554 & 0.9591 & 0.9625 \\
\hline & 35 & 0.9456 & 0.9485 & 0.9521 & 0.9551 & 0.9589 & 0.9613 \\
\hline & 40 & 0.9421 & 0.9459 & 0.9494 & 0.9531 & 0.9545 & 0.9567 \\
\hline & 45 & 0.9403 & 0.9420 & 0.9451 & 0.9488 & 0.9509 & 0.9539 \\
\hline & 50 & 0.9449 & 0.9469 & 0.9484 & 0.9507 & 0.9534 & 0.9561 \\
\hline \multirow{7}{*}{-0.1} & 20 & 0.9382 & 0.9446 & 0.9499 & 0.9567 & 0.9624 & $\overline{0.9676}$ \\
\hline & 25 & 0.9412 & 0.9456 & 0.9507 & 0.9555 & 0.9610 & 0.9659 \\
\hline & 30 & 0.9423 & 0.9466 & 0.9496 & 0.9541 & 0.9570 & 0.9618 \\
\hline & 35 & 0.9448 & 0.9481 & 0.9520 & 0.9546 & 0.9574 & 0.9604 \\
\hline & 40 & 0.9462 & 0.9487 & 0.9511 & 0.9545 & 0.9573 & 0.9598 \\
\hline & 45 & 0.9427 & 0.9455 & 0.9482 & 0.9505 & 0.9541 & 0.9568 \\
\hline & 50 & 0.9444 & 0.9465 & 0.9485 & 0.9503 & 0.9521 & 0.9549 \\
\hline \multirow{7}{*}{0} & 20 & 0.9400 & 0.9461 & 0.9514 & 0.9565 & 0.9616 & 0.9680 \\
\hline & 25 & 0.9419 & 0.9462 & 0.9517 & 0.9552 & 0.9602 & 0.9650 \\
\hline & 30 & 0.9420 & 0.9455 & 0.9479 & 0.9518 & 0.9559 & 0.9592 \\
\hline & 35 & 0.9432 & 0.9468 & 0.9499 & 0.9540 & 0.9573 & 0.9608 \\
\hline & 40 & 0.9413 & 0.9444 & 0.9479 & 0.9504 & 0.9534 & 0.9568 \\
\hline & 45 & 0.9463 & 0.9493 & 0.9519 & 0.9547 & 0.9574 & 0.9596 \\
\hline & 50 & 0.9439 & 0.9469 & 0.9485 & 0.9506 & 0.9521 & 0.9547 \\
\hline \multirow{7}{*}{0.1} & 20 & 0.9366 & 0.9422 & 0.9496 & 0.9557 & 0.9627 & 0.9686 \\
\hline & 25 & 0.9399 & 0.9442 & 0.9496 & 0.9551 & 0.9606 & 0.9657 \\
\hline & 30 & 0.9427 & 0.9468 & 0.9512 & 0.9544 & 0.9586 & 0.9626 \\
\hline & 35 & 0.9397 & 0.9424 & 0.9463 & 0.9495 & 0.9537 & 0.9567 \\
\hline & 40 & 0.9444 & 0.9478 & 0.9494 & 0.9525 & 0.9551 & 0.9579 \\
\hline & 45 & 0.9440 & 0.9478 & 0.9505 & 0.9534 & 0.9575 & 0.9593 \\
\hline & 50 & 0.9436 & 0.9455 & 0.9482 & 0.9507 & 0.9526 & 0.9552 \\
\hline \multirow{7}{*}{0.2} & 20 & 0.9388 & 0.9445 & 0.9521 & 0.9560 & 0.9618 & 0.9676 \\
\hline & 25 & 0.9409 & 0.9467 & 0.9531 & 0.9573 & 0.9616 & 0.9648 \\
\hline & 30 & 0.9399 & 0.9433 & 0.9465 & 0.9500 & 0.9539 & 0.9583 \\
\hline & 35 & 0.9420 & 0.9457 & 0.9497 & 0.9525 & 0.9557 & 0.9585 \\
\hline & 40 & 0.9442 & 0.9479 & 0.9504 & 0.9536 & 0.9568 & 0.9588 \\
\hline & 45 & 0.9451 & 0.9480 & 0.9500 & 0.9535 & 0.9557 & 0.9579 \\
\hline & 50 & 0.9427 & 0.9451 & 0.9476 & 0.9509 & 0.9524 & 0.9554 \\
\hline \multirow{7}{*}{0.3} & 20 & 0.9352 & 0.9408 & 0.9478 & 0.9557 & 0.9625 & 0.9687 \\
\hline & 25 & 0.9439 & 0.9497 & 0.9537 & 0.9582 & 0.9627 & 0.9663 \\
\hline & 30 & 0.9420 & 0.9459 & 0.9507 & 0.9547 & 0.9584 & 0.9617 \\
\hline & 35 & 0.9458 & 0.9488 & 0.9519 & 0.9547 & 0.9578 & 0.9615 \\
\hline & 40 & 0.9437 & 0.9465 & 0.9498 & 0.9520 & 0.9536 & 0.9574 \\
\hline & 45 & 0.9444 & 0.9483 & 0.9501 & 0.9524 & 0.9547 & 0.9570 \\
\hline & 50 & 0.9475 & 0.9497 & 0.9513 & 0.9541 & 0.9562 & 0.9593 \\
\hline \multirow{7}{*}{0.4} & 20 & 0.9356 & 0.9425 & 0.9490 & 0.9544 & 0.9610 & 0.9660 \\
\hline & 25 & 0.9397 & 0.9439 & 0.9492 & 0.9532 & 0.9569 & 0.9606 \\
\hline & 30 & 0.9414 & 0.9458 & 0.9499 & 0.9531 & 0.9577 & 0.9627 \\
\hline & 35 & 0.9447 & 0.9472 & 0.9507 & 0.9543 & 0.9568 & 0.9591 \\
\hline & 40 & 0.9445 & 0.9477 & 0.9513 & 0.9540 & 0.9567 & 0.9591 \\
\hline & 45 & 0.9412 & 0.9449 & 0.9471 & 0.9498 & 0.9523 & 0.9550 \\
\hline & 50 & 0.9425 & 0.9455 & 0.9483 & 0.9506 & 0.9528 & 0.9544 \\
\hline
\end{tabular}

Table 4.8: Comparison Denominadors MLE using Fisher Transformation 


\begin{tabular}{|c|c|c|c|c|c|c|c|}
\hline & & \multicolumn{6}{|c|}{ DENOMINADOR } \\
\hline RHO & $\begin{array}{c}\text { SAMPLE } \\
\text { SIZE }\end{array}$ & $\mathbf{n}$ & $\mathbf{n - 1}$ & $\mathbf{n}-\mathbf{2}$ & $\mathbf{n}-\mathbf{3}$ & $\mathbf{n}-\mathbf{4}$ & $\mathbf{n}-\mathbf{5}$ \\
\hline & $\mathbf{2 0}$ & 0.9376 & 0.9428 & 0.9486 & 0.9556 & 0.9612 & 0.9678 \\
& $\mathbf{2 5}$ & 0.9417 & 0.9477 & 0.9519 & 0.9570 & 0.9617 & 0.9664 \\
& $\mathbf{3 0}$ & 0.9422 & 0.9470 & 0.9514 & 0.9551 & 0.9589 & 0.9617 \\
$\mathbf{0 . 5}$ & $\mathbf{3 5}$ & 0.9418 & 0.9449 & 0.9492 & 0.9538 & 0.9562 & 0.9593 \\
& $\mathbf{4 0}$ & 0.9409 & 0.9439 & 0.9479 & 0.9514 & 0.9554 & 0.9577 \\
& $\mathbf{4 5}$ & 0.9443 & 0.9477 & 0.9502 & 0.9529 & 0.9559 & 0.9578 \\
& $\mathbf{5 0}$ & 0.9451 & 0.9478 & 0.9503 & 0.9524 & 0.9550 & 0.9563 \\
\hline & $\mathbf{2 0}$ & 0.9366 & 0.9438 & 0.9495 & 0.9551 & 0.9616 & 0.9678 \\
& $\mathbf{2 5}$ & 0.9396 & 0.9449 & 0.9500 & 0.9540 & 0.9585 & 0.9632 \\
& $\mathbf{3 0}$ & 0.9418 & 0.9455 & 0.9493 & 0.9542 & 0.9586 & 0.9626 \\
$\mathbf{0 . 6}$ & $\mathbf{3 5}$ & 0.9443 & 0.9480 & 0.9519 & 0.9556 & 0.9582 & 0.9617 \\
& $\mathbf{4 0}$ & 0.9473 & 0.9498 & 0.9520 & 0.9550 & 0.9581 & 0.9607 \\
& $\mathbf{4 5}$ & 0.9446 & 0.9483 & 0.9512 & 0.9537 & 0.9564 & 0.9590 \\
& $\mathbf{5 0}$ & 0.9456 & 0.9484 & 0.9500 & 0.9516 & 0.9543 & 0.9562 \\
\hline & $\mathbf{2 0}$ & 0.9409 & 0.9482 & 0.9536 & 0.9587 & 0.9632 & 0.9696 \\
& $\mathbf{2 5}$ & 0.9415 & 0.9458 & 0.9517 & 0.9567 & 0.9608 & 0.9649 \\
& $\mathbf{3 0}$ & 0.9441 & 0.9490 & 0.9538 & 0.9568 & 0.9602 & 0.9636 \\
$\mathbf{0 . 7}$ & $\mathbf{3 5}$ & 0.9458 & 0.9491 & 0.9520 & 0.9552 & 0.9584 & 0.9625 \\
& $\mathbf{4 0}$ & 0.9420 & 0.9455 & 0.9481 & 0.9507 & 0.9536 & 0.9565 \\
& $\mathbf{4 5}$ & 0.9458 & 0.9493 & 0.9512 & 0.9539 & 0.9559 & 0.9594 \\
& $\mathbf{5 0}$ & 0.9445 & 0.9464 & 0.9479 & 0.9507 & 0.9529 & 0.9558 \\
\hline
\end{tabular}

\begin{tabular}{|c|c|c|c|c|c|c|c|}
\hline & & \multicolumn{6}{|c|}{ DENOMINADOR } \\
\hline RHO & $\begin{array}{c}\text { SAMPLE } \\
\text { SIZE }\end{array}$ & $\mathbf{n}$ & $\mathbf{n}-\mathbf{1}$ & $\mathbf{n}-\mathbf{2}$ & $\mathbf{n}-\mathbf{3}$ & $\mathbf{n}-\mathbf{4}$ & $\mathbf{n}-\mathbf{5}$ \\
\hline \multirow{6}{*}{$\mathbf{2 0}$} & 0.9396 & 0.9477 & 0.9530 & 0.9583 & 0.9647 & 0.9700 \\
& $\mathbf{2 5}$ & 0.9346 & 0.9401 & 0.9443 & 0.9499 & 0.9546 & 0.9601 \\
& $\mathbf{3 0}$ & 0.9420 & 0.9475 & 0.9509 & 0.9549 & 0.9601 & 0.9635 \\
& $\mathbf{3 5}$ & 0.9425 & 0.9467 & 0.9494 & 0.9535 & 0.9571 & 0.9594 \\
& $\mathbf{4 0}$ & 0.9451 & 0.9479 & 0.9513 & 0.9541 & 0.9571 & 0.9590 \\
& $\mathbf{4 5}$ & 0.9444 & 0.9476 & 0.9499 & 0.9531 & 0.9550 & 0.9573 \\
& $\mathbf{5 0}$ & 0.9470 & 0.9488 & 0.9508 & 0.9534 & 0.9558 & 0.9576 \\
\hline \multirow{4}{*}{$\mathbf{0 . 9}$} & 0.9336 & 0.9393 & 0.9474 & 0.9547 & 0.9612 & 0.9668 \\
& $\mathbf{2 0}$ & 0.9384 & 0.9430 & 0.9478 & 0.9530 & 0.9574 & 0.9631 \\
& $\mathbf{2 0}$ & 0.9415 & 0.9452 & 0.9493 & 0.9540 & 0.9585 & 0.9624 \\
& $\mathbf{3 5}$ & 0.9419 & 0.9449 & 0.9479 & 0.9514 & 0.9563 & 0.9601 \\
& $\mathbf{4 0}$ & 0.9433 & 0.9463 & 0.9485 & 0.9511 & 0.9539 & 0.9566 \\
& $\mathbf{4 5}$ & 0.9471 & 0.9495 & 0.9516 & 0.9537 & 0.9562 & 0.9591 \\
& $\mathbf{5 0}$ & 0.9437 & 0.9470 & 0.9489 & 0.9509 & 0.9531 & 0.9543 \\
\hline
\end{tabular}

Table 4.9: Cont. Comparison Denominadors MLE using Fisher Transformation

Tables 4.8 and 4.9 include the coverages of the intervals using the MLE. 

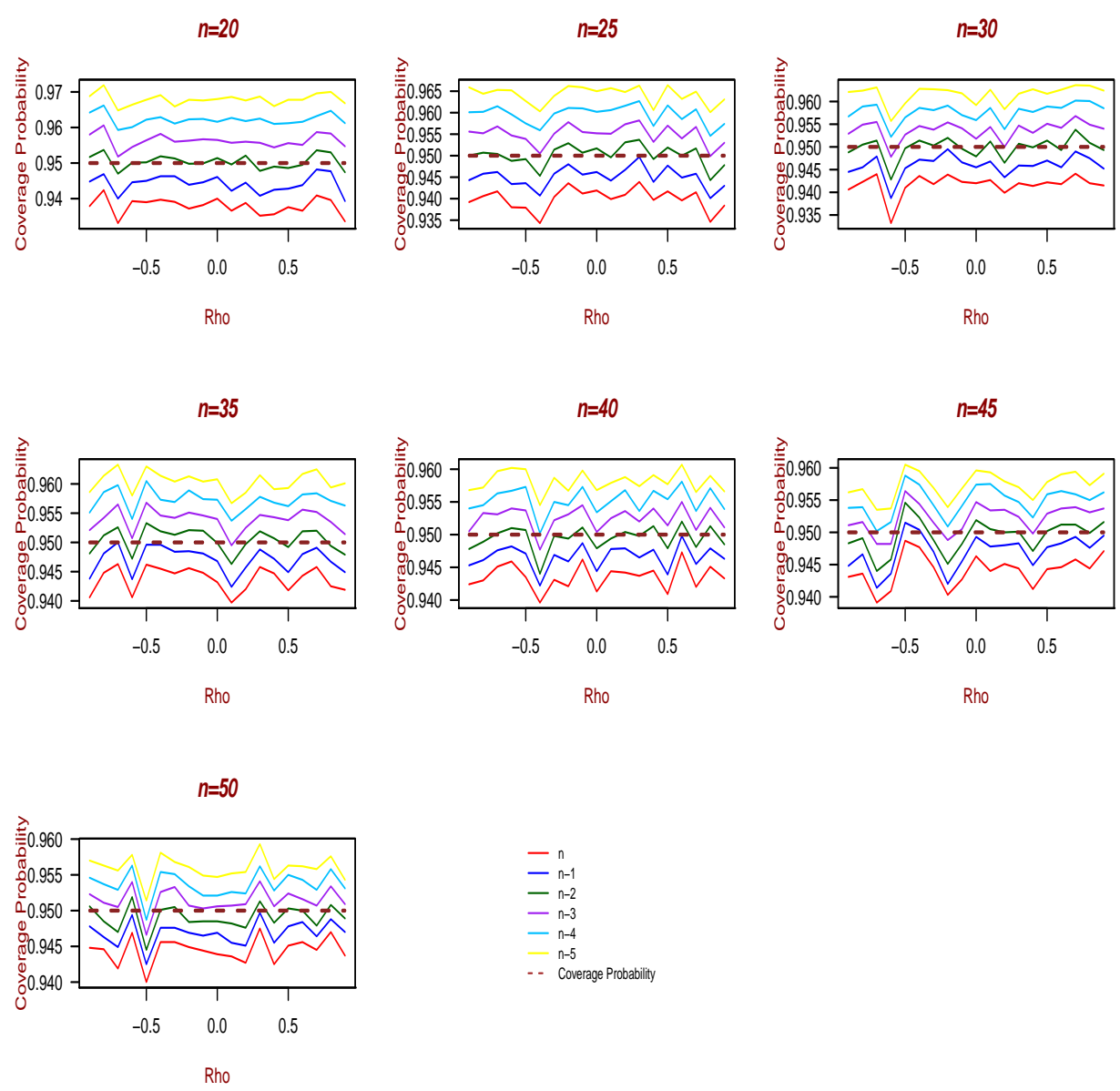

Figure 4.7: Comparison Denominators for MLE using Fisher Transformation

In Figure 4.7 is easily observable that for $k=2$, the $95 \%$ level is achieved. Therefore, it can be conclude that

$$
\frac{1}{2} \ln \left(\frac{1+\hat{\rho}_{n}}{1-\hat{\rho}_{n}}\right) \sim N\left(\frac{1}{2} \ln \left(\frac{1+\rho}{1-\rho}\right), \frac{1}{n-2}\right)
$$

is more appropiate. 
Table 4.10: Comparison Denominadors Pearson using Fisher Dist.

\begin{tabular}{|c|c|c|c|c|c|c|c|}
\hline \multirow[b]{2}{*}{ RHO } & \multirow[b]{2}{*}{$\begin{array}{c}\text { SAMPLE } \\
\text { SIZE }\end{array}$} & \multicolumn{6}{|c|}{ DENOMINADOR } \\
\hline & & $\mathbf{n}$ & $n-1$ & $\mathrm{n}-2$ & $n-3$ & $n-4$ & $n-5$ \\
\hline \multirow{7}{*}{-0.9} & 20 & 0.9301 & 0.9361 & 0.9437 & 0.9522 & 0.9588 & 0.9638 \\
\hline & 25 & 0.9343 & 0.9405 & 0.9447 & 0.9508 & 0.9554 & 0.9603 \\
\hline & 30 & 0.9353 & 0.9387 & 0.9442 & 0.9489 & 0.9543 & 0.9587 \\
\hline & 35 & 0.9374 & 0.9421 & 0.9455 & 0.9491 & 0.9521 & 0.9564 \\
\hline & 40 & 0.9396 & 0.9427 & 0.9465 & 0.9488 & 0.9517 & 0.9551 \\
\hline & 45 & 0.9417 & 0.9443 & 0.9465 & 0.9497 & 0.9522 & 0.9545 \\
\hline & 50 & 0.9435 & 0.9458 & 0.9482 & 0.9501 & 0.9521 & 0.9551 \\
\hline \multirow{7}{*}{-0.8} & 20 & 0.9362 & 0.9420 & 0.9486 & 0.9556 & 0.9614 & 0.9676 \\
\hline & 25 & 0.9359 & 0.9404 & 0.9465 & 0.9510 & 0.9561 & 0.9605 \\
\hline & 30 & 0.9390 & 0.9434 & 0.9481 & 0.9521 & 0.9567 & 0.9610 \\
\hline & 35 & 0.9435 & 0.9463 & 0.9490 & 0.9521 & 0.9553 & 0.9583 \\
\hline & 40 & 0.9396 & 0.9425 & 0.9466 & 0.9493 & 0.9525 & 0.9551 \\
\hline & 45 & 0.9415 & 0.9445 & 0.9478 & 0.9504 & 0.9528 & 0.9548 \\
\hline & 50 & 0.9427 & 0.9447 & 0.9464 & 0.9495 & 0.9511 & 0.9527 \\
\hline \multirow{7}{*}{-0.7} & 20 & 0.9249 & 0.9318 & 0.9384 & 0.9462 & 0.9532 & 0.9600 \\
\hline & 25 & 0.9356 & 0.9413 & 0.9460 & 0.9519 & 0.9567 & 0.9612 \\
\hline & 30 & 0.9382 & 0.9424 & 0.9476 & 0.9514 & 0.9559 & 0.9604 \\
\hline & 35 & 0.9420 & 0.9456 & 0.9492 & 0.9529 & 0.9567 & 0.9603 \\
\hline & 40 & 0.9427 & 0.9452 & 0.9483 & 0.9511 & 0.9537 & 0.9573 \\
\hline & 45 & 0.9370 & 0.9396 & 0.9421 & 0.9458 & 0.9491 & 0.9518 \\
\hline & 50 & 0.9400 & 0.9422 & 0.9449 & 0.9476 & 0.9500 & 0.9526 \\
\hline \multirow{7}{*}{-0.6} & 2 & 0.9319 & 0.9376 & 0.9427 & 0.9494 & 0.9555 & 0.9616 \\
\hline & 25 & 0.9340 & 0.9392 & 0.9440 & 0.9510 & 0.9552 & 0.9610 \\
\hline & 30 & 0.9287 & 0.9329 & 0.9385 & 0.9431 & 0.9478 & 0.9518 \\
\hline & 35 & 0.9369 & 0.9395 & 0.9435 & 0.9470 & 0.9508 & 0.9551 \\
\hline & 40 & 0.9441 & 0.9470 & 0.9491 & 0.9520 & 0.9543 & 0.9580 \\
\hline & 45 & 0.9377 & 0.9400 & 0.9430 & 0.9461 & 0.9489 & 0.9515 \\
\hline & 50 & 0.9441 & 0.9464 & 0.9490 & 0.95 & 0.9527 & 0.9554 \\
\hline \multirow{7}{*}{-0.5} & 20 & 0.9306 & 0.9380 & 0.9450 & 0.95 & 0.9577 & 0.9628 \\
\hline & 25 & 0.9334 & 0.9383 & 0.9433 & 0.9488 & 0.9536 & 0.9585 \\
\hline & 30 & 0.9362 & 0.9413 & 0.9451 & 0.9487 & 0.9522 & 0.9564 \\
\hline & 35 & 0.9431 & 0.9459 & 0.9489 & 0.9534 & 0.9570 & 0.9599 \\
\hline & 40 & 0.94 & 0.9444 & 0.9477 & 0.95 & 0.9541 & 0.9573 \\
\hline & 45 & 0.9452 & 0.9490 & 0.9520 & 0.9544 & 0.9563 & 0.9595 \\
\hline & 50 & 0.9381 & 0.9407 & 0.9436 & 0.9456 & 0.9478 & 0.9503 \\
\hline \multirow{7}{*}{-0.4} & 2 & 0.9322 & 0.9395 & 0.9459 & 0.9525 & 0.9580 & 0.9638 \\
\hline & 25 & 0.930 & 0.9358 & 0.9412 & 0.94 & 0.9519 & 0.9569 \\
\hline & 30 & 0.9383 & 0.9436 & 0.9483 & 0.9513 & 0.9551 & 0.9593 \\
\hline & 35 & 0.9408 & 0.9451 & 0.9478 & 0.9517 & 0.9540 & 0.9582 \\
\hline & 40 & 0.9362 & 0.9393 & 0.9423 & 0.9456 & 0.9474 & 0.9509 \\
\hline & 45 & 0.9450 & 0.9474 & 0.9493 & 0.9519 & 0.9550 & 0.9574 \\
\hline & 50 & 9421 & 0.9443 & 0.9476 & 0.9502 & 0.9530 & 0.9556 \\
\hline \multirow{7}{*}{-0.3} & 20 & 0.9317 & 0.9384 & 0.9455 & 0.9506 & 0.9564 & 0.9612 \\
\hline & 25 & 0.9340 & 0.9407 & 0.9452 & 0.9499 & 0.9551 & 0.9599 \\
\hline & 30 & 0.9366 & 0.9417 & 0.9453 & 0.9493 & 0.9534 & 0.9589 \\
\hline & 35 & 0.9416 & 0.9450 & 0.9480 & 0.9514 & 0.9542 & 0.9579 \\
\hline & 40 & 0.9392 & 0.9434 & 0.9464 & 0.9492 & 0.9517 & 0.9550 \\
\hline & 45 & 0.9419 & 0.9444 & 0.9466 & 0.9494 & 0.9516 & 0.9541 \\
\hline & 50 & 0.9434 & 0.9456 & 0.9482 & 0.9511 & 0.9537 & 0.9553 \\
\hline
\end{tabular}

\begin{tabular}{|c|c|c|c|c|c|c|c|}
\hline \multirow[b]{2}{*}{ RHO } & \multirow[b]{2}{*}{$\begin{array}{c}\text { SAMPLE } \\
\text { SIZE }\end{array}$} & \multicolumn{6}{|c|}{ DENOMINADOR } \\
\hline & & $\mathbf{n}$ & $n-1$ & $\mathrm{n}-2$ & $n-3$ & $n-4$ & $n-5$ \\
\hline \multirow{7}{*}{-0.2} & 20 & 0.9315 & 0.9370 & 0.9442 & 0.9502 & 0.9571 & 0.9630 \\
\hline & 25 & 0.9390 & 0.9431 & 0.9475 & 0.9530 & 0.9563 & 0.9626 \\
\hline & 30 & 0.9395 & 0.9450 & 0.9487 & 0.9527 & 0.9559 & 0.9589 \\
\hline & 35 & 0.9416 & 0.9458 & 0.9491 & 0.9516 & 0.9553 & 0.9578 \\
\hline & 40 & 0.9385 & 0.9418 & 0.9458 & 0.9497 & 0.9513 & 0.9536 \\
\hline & 45 & 0.9384 & 0.9398 & 0.9418 & 0.9456 & 0.9486 & 0.9514 \\
\hline & 50 & 0.9431 & 0.9452 & 0.9464 & 0.9483 & 0.9515 & 0.9542 \\
\hline \multirow{7}{*}{-0.1} & 20 & 0.9308 & 0.9371 & 0.9438 & 0.9495 & 0.9570 & 0.9628 \\
\hline & 25 & 0.9346 & 0.9402 & 0.9452 & 0.9507 & 0.9553 & 0.9619 \\
\hline & 30 & 0.9364 & 0.9416 & 0.9450 & 0.9500 & 0.9540 & 0.9587 \\
\hline & 35 & 0.9420 & 0.9448 & 0.9477 & 0.9513 & 0.9542 & 0.9582 \\
\hline & 40 & 0.9415 & 0.9450 & 0.9485 & 0.9515 & 0.9546 & 0.9573 \\
\hline & 45 & 0.9400 & 0.9431 & 0.9457 & 0.9479 & 0.9512 & 0.9546 \\
\hline & 50 & 0.9415 & 0.9439 & 0.9464 & 0.9487 & 0.9504 & 0.9524 \\
\hline \multirow{7}{*}{0} & 20 & 0.9326 & 0.9393 & 0.9458 & 0.9521 & 0.9574 & 0.9632 \\
\hline & 25 & 0.9369 & 0.9412 & 0.9470 & 0.9511 & 0.9570 & 0.9616 \\
\hline & 30 & 0.9367 & 0.9412 & 0.9453 & 0.9484 & 0.9518 & 0.9555 \\
\hline & 35 & 0.9393 & 0.9437 & 0.9474 & 0.9504 & 0.9545 & 0.9582 \\
\hline & 40 & 0.9385 & 0.9414 & 0.9449 & 0.9474 & 0.9504 & 0.9533 \\
\hline & 45 & 0.9441 & 0.9469 & 0.9488 & 0.9519 & 0.9550 & 0.9573 \\
\hline & 50 & 0.9421 & 0.9439 & 0.9466 & 0.9492 & 0.9507 & 0.9526 \\
\hline \multirow{7}{*}{0.1} & 20 & 0.9287 & 0.9342 & 0.9415 & 0.9492 & 0.9568 & 0.9625 \\
\hline & 25 & 0.9335 & 0.9378 & 0.9435 & 0.9490 & 0.9549 & 0.9606 \\
\hline & 30 & 0.9381 & 0.9425 & 0.9470 & 0.9501 & 0.9542 & 0.9584 \\
\hline & 35 & 0.9353 & 0.9376 & 0.9423 & 0.9464 & 0.9509 & 0.9534 \\
\hline & 40 & 0.9415 & 0.9444 & 0.9471 & 0.9497 & 0.9523 & 0.9550 \\
\hline & 45 & 0.9405 & 0.9446 & 0.9476 & 0.9505 & 0.9535 & 0.9566 \\
\hline & 50 & 0.9414 & 0.9437 & 0.9453 & 0.9475 & 0.9505 & 0.9530 \\
\hline \multirow{7}{*}{0.2} & 20 & 0.9308 & 0.9373 & 0.9446 & 0.9495 & 0.9569 & 0.9631 \\
\hline & 25 & 0.9363 & 0.9417 & 0.9469 & 0.9518 & 0.9575 & 0.9614 \\
\hline & 30 & 0.9357 & 0.9387 & 0.9423 & 0.9464 & 0.9503 & 0.9549 \\
\hline & 35 & 0.9382 & 0.9417 & 0.9459 & 0.9500 & 0.9534 & 0.9566 \\
\hline & 40 & 0.9418 & 0.9452 & 0.9476 & 0.9513 & 0.9548 & 0.9572 \\
\hline & 45 & 0.9423 & 0.9460 & 0.9477 & 0.9509 & 0.9536 & 0.9558 \\
\hline & 50 & 0.9405 & 0.9427 & 0.9454 & 0.9474 & 0.9498 & 0.9524 \\
\hline \multirow{7}{*}{0.3} & 20 & 0.9287 & 0.9335 & 0.9404 & 0.9487 & 0.9565 & 0.9633 \\
\hline & 25 & 0.9393 & 0.9450 & 0.9483 & 0.9540 & 0.9593 & 0.9625 \\
\hline & 30 & 0.9373 & 0.9426 & 0.9464 & 0.9510 & 0.9559 & 0.9585 \\
\hline & 35 & 0.9422 & 0.9457 & 0.9490 & 0.9522 & 0.9557 & 0.9589 \\
\hline & 40 & 0.9408 & 0.9439 & 0.9462 & 0.9490 & 0.9507 & 0.9554 \\
\hline & 45 & 0.9397 & 0.9437 & 0.9465 & 0.9491 & 0.9516 & 0.9540 \\
\hline & 50 & 0.9441 & 0.9469 & 0.9495 & 0.9516 & 0.9538 & 0.9567 \\
\hline \multirow{7}{*}{0.4} & 20 & 0.9298 & 0.9360 & 0.9431 & 0.9500 & 0.9557 & 0.9617 \\
\hline & 25 & 0.9332 & 0.9392 & 0.9445 & 0.9483 & 0.9523 & 0.9558 \\
\hline & 30 & 0.9364 & 0.9403 & 0.9449 & 0.9491 & 0.9528 & 0.9589 \\
\hline & 35 & 0.9411 & 0.9446 & 0.9478 & 0.9510 & 0.9547 & 0.9572 \\
\hline & 40 & 0.9401 & 0.9449 & 0.9481 & 0.9510 & 0.9536 & 0.9566 \\
\hline & 45 & 0.9380 & 0.9415 & 0.9443 & 0.9473 & 0.9499 & 0.9527 \\
\hline & 50 & 0.9402 & 0.9430 & 0.9458 & 0.9488 & 0.9507 & 0.9526 \\
\hline
\end{tabular}


Table 4.11: Cont. Comparison Denominadors Pearson using Fisher Dist.

\begin{tabular}{|r|c|r|r|r|r|r|r|}
\hline & & \multicolumn{6}{|c|}{ DENOMINADOR } \\
\hline RHO & $\begin{array}{c}\text { SAMPLE } \\
\text { SIZE }\end{array}$ & $\mathbf{n}$ & $\mathbf{n}-\mathbf{1}$ & $\mathbf{n}-\mathbf{2}$ & $\mathbf{n}-\mathbf{3}$ & $\mathbf{n}-\mathbf{4}$ & $\mathbf{n}-\mathbf{5}$ \\
\hline & $\mathbf{2 0}$ & 0.9312 & 0.9369 & 0.9433 & 0.9498 & 0.9561 & 0.9626 \\
& $\mathbf{2 5}$ & 0.9369 & 0.9425 & 0.9471 & 0.9528 & 0.9575 & 0.9621 \\
& $\mathbf{3 0}$ & 0.9379 & 0.9429 & 0.9477 & 0.9517 & 0.9549 & 0.9586 \\
$\mathbf{0 . 5}$ & $\mathbf{3 5}$ & 0.9385 & 0.9422 & 0.9458 & 0.9498 & 0.9534 & 0.9567 \\
& $\mathbf{4 0}$ & 0.9373 & 0.9407 & 0.9447 & 0.9474 & 0.9514 & 0.955 \\
& $\mathbf{4 5}$ & 0.941 & 0.9437 & 0.9469 & 0.9499 & 0.9531 & 0.9555 \\
& $\mathbf{5 0}$ & 0.9433 & 0.9453 & 0.948 & 0.9507 & 0.9525 & 0.9545 \\
\hline & $\mathbf{2 0}$ & 0.9295 & 0.9363 & 0.9438 & 0.9503 & 0.9559 & 0.9627 \\
& $\mathbf{2 5}$ & 0.9358 & 0.9415 & 0.9464 & 0.9505 & 0.9550 & 0.9597 \\
& $\mathbf{3 0}$ & 0.9390 & 0.9421 & 0.9461 & 0.9511 & 0.9557 & 0.9596 \\
$\mathbf{0 . 6}$ & $\mathbf{3 5}$ & 0.9413 & 0.9456 & 0.9503 & 0.9531 & 0.9559 & 0.9594 \\
& $\mathbf{4 0}$ & 0.9450 & 0.9474 & 0.9492 & 0.9521 & 0.9549 & 0.9578 \\
& $\mathbf{4 5}$ & 0.9430 & 0.9459 & 0.9484 & 0.9509 & 0.9535 & 0.9565 \\
& $\mathbf{5 0}$ & 0.9434 & 0.946 & 0.9487 & 0.9501 & 0.9525 & 0.9545 \\
\hline & $\mathbf{2 0}$ & 0.9362 & 0.943 & 0.9492 & 0.9556 & 0.9605 & 0.9659 \\
& $\mathbf{2 5}$ & 0.9378 & 0.9435 & 0.9483 & 0.9526 & 0.9567 & 0.9606 \\
& $\mathbf{3 0}$ & 0.9403 & 0.9457 & 0.949 & 0.9522 & 0.9573 & 0.9615 \\
$\mathbf{0 . 7}$ & $\mathbf{3 5}$ & 0.9421 & 0.9454 & 0.9496 & 0.9531 & 0.9568 & 0.9601 \\
& $\mathbf{4 0}$ & 0.9384 & 0.942 & 0.9454 & 0.9483 & 0.9519 & 0.9543 \\
& $\mathbf{4 5}$ & 0.9440 & 0.9462 & 0.9489 & 0.9504 & 0.9532 & 0.956 \\
& $\mathbf{5 0}$ & 0.9411 & 0.9427 & 0.9453 & 0.9483 & 0.9507 & 0.9539 \\
\hline
\end{tabular}

\begin{tabular}{|l|c|r|r|r|r|r|c|}
\hline & & \multicolumn{6}{|c|}{ DENOMINADOR } \\
\hline RHO & $\begin{array}{c}\text { SAMPLE } \\
\text { SIZE }\end{array}$ & $\mathbf{n}$ & $\mathbf{n}-\mathbf{1}$ & $\mathbf{n - 2}$ & $\mathbf{n}-\mathbf{3}$ & $\mathbf{n}-\mathbf{4}$ & $\mathbf{n}-\mathbf{5}$ \\
\hline & $\mathbf{2 0}$ & 0.9325 & 0.9381 & 0.9448 & 0.9514 & 0.958 & 0.9639 \\
& $\mathbf{2 5}$ & 0.9298 & 0.935 & 0.9407 & 0.9455 & 0.9504 & 0.9543 \\
& $\mathbf{3 0}$ & 0.9380 & 0.9427 & 0.9470 & 0.9513 & 0.9555 & 0.9595 \\
$\mathbf{0 . 8}$ & $\mathbf{3 5}$ & 0.9373 & 0.9415 & 0.9449 & 0.9498 & 0.9543 & 0.9562 \\
& $\mathbf{4 0}$ & 0.941 & 0.9448 & 0.9478 & 0.9506 & 0.9534 & 0.9567 \\
& $\mathbf{4 5}$ & 0.9433 & 0.9456 & 0.9485 & 0.95 & 0.952 & 0.9554 \\
& $\mathbf{5 0}$ & 0.9436 & 0.9467 & 0.9488 & 0.9515 & 0.9547 & 0.9577 \\
\hline & $\mathbf{2 0}$ & 0.9295 & 0.9362 & 0.9436 & 0.9513 & 0.9564 & 0.9619 \\
& $\mathbf{2 5}$ & 0.9342 & 0.9399 & 0.9442 & 0.9486 & 0.9542 & 0.9604 \\
& $\mathbf{3 0}$ & 0.9353 & 0.9399 & 0.9443 & 0.9490 & 0.9545 & 0.9584 \\
$\mathbf{0 . 9}$ & $\mathbf{3 5}$ & 0.9395 & 0.9429 & 0.9465 & 0.9497 & 0.954 & 0.9574 \\
& $\mathbf{4 0}$ & 0.9403 & 0.9439 & 0.9465 & 0.9496 & 0.9524 & 0.9551 \\
& $\mathbf{4 5}$ & 0.9464 & 0.9488 & 0.9508 & 0.9528 & 0.9554 & 0.9580 \\
& $\mathbf{5 0}$ & 0.9416 & 0.9441 & 0.9466 & 0.9484 & 0.9505 & 0.9529 \\
\hline
\end{tabular}



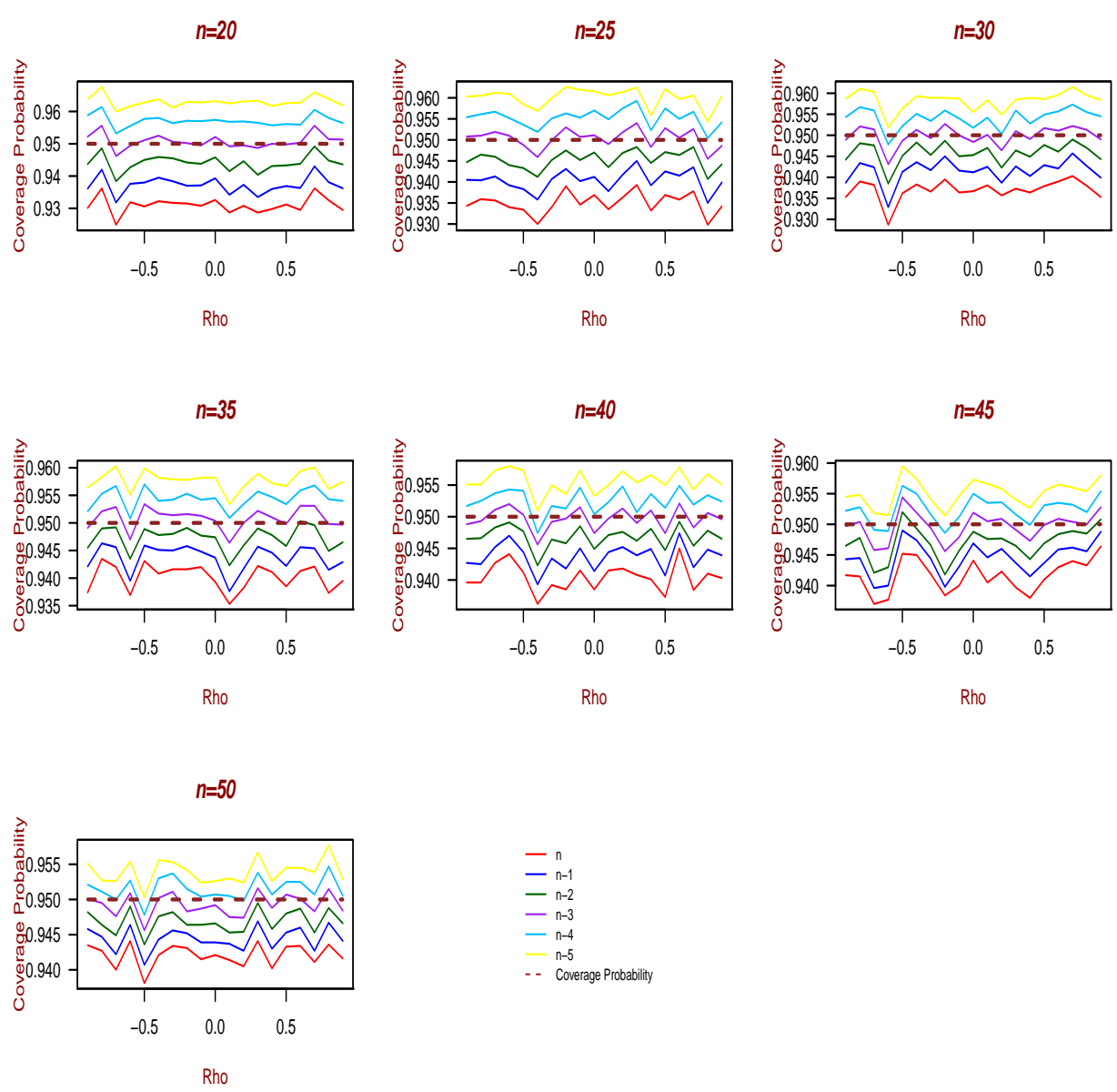

Figure 4.8: Comparison Denominators for Pearson Estimator using Fisher Transformation

The coverage probability for the confidences intervals in expression (4.4) using the Pearson Correlation Coefficient is represented Figure 4.8 whose values are presented in Tables 4.10 and 4.11 . It can be seen that $n-3$ is the best denominator when the Pearson Correlation Coefficient $\hat{r}$ is used as estimator. Therefore,

$$
\frac{1}{2} \ln \left(\frac{1+\hat{r}_{n}}{1-\hat{r}_{n}}\right) \sim N\left(\frac{1}{2} \ln \left(\frac{1+\rho}{1-\rho}\right), \frac{1}{n-3}\right)
$$




\subsubsection{Confidence Intervals}

In previous section it was shown that the for the MLE $\hat{\rho}_{n}$

$$
\frac{1}{2} \ln \left(\frac{1+\hat{\rho}_{n}}{1-\hat{\rho}_{n}}\right) \sim N\left(\frac{1}{2} \ln \left(\frac{1+\rho}{1-\rho}\right), \frac{1}{n-2}\right)
$$

and for the Pearson correlation coefficient $\hat{r}_{n}$

$$
\frac{1}{2} \ln \left(\frac{1+\hat{r}_{n}}{1-\hat{r}_{n}}\right) \sim N\left(\frac{1}{2} \ln \left(\frac{1+\rho}{1-\rho}\right), \frac{1}{n-3}\right) .
$$

and thus, a confidence interval for $\rho$ can be found.

In this section a comparison between those intervals will be performed. For our purpose, the coverage probability and the average width of the intervals were recorded and showed in the next figures for (a) the MLE and (b) the Pearson Correlation Coefficient. 
Table 4.12: Comparison Coverage Probability

\begin{tabular}{|c|c|c|c|c|c|c|c|c|}
\hline & \multirow{2}{*}{ Rho } & \multicolumn{7}{|c|}{ SAMPLE SIZE } \\
\hline & & 20 & 25 & 30 & 35 & 40 & 45 & 50 \\
\hline \multirow{19}{*}{$\sqrt{0}$} & -0.9 & 0.9517 & 0.9500 & 0.9488 & 0.9481 & 0.9478 & 0.9483 & 0.9506 \\
\hline & -0.8 & 0.9537 & 0.9507 & 0.9505 & 0.9512 & 0.9489 & 0.9491 & 0.9485 \\
\hline & -0.7 & 0.9470 & 0.9504 & 0.9514 & 0.9526 & 0.9502 & 0.9440 & 0.9470 \\
\hline & -0.6 & 0.9501 & 0.9488 & 0.9428 & 0.9472 & 0.9510 & 0.9458 & 0.9519 \\
\hline & -0.5 & 0.9502 & 0.9492 & 0.9497 & 0.9533 & 0.9507 & 0.9546 & 0.9445 \\
\hline & -0.4 & 0.9519 & 0.9453 & 0.9514 & 0.9519 & 0.9440 & 0.9523 & 0.9501 \\
\hline & -0.3 & 0.9513 & 0.9514 & 0.9503 & 0.9513 & 0.9498 & 0.9488 & 0.9505 \\
\hline & -0.2 & 0.9498 & 0.9529 & 0.9520 & 0.9521 & 0.9494 & 0.9451 & 0.9484 \\
\hline & -0.1 & 0.9499 & 0.9507 & 0.9496 & 0.9520 & 0.9511 & 0.9482 & 0.9485 \\
\hline & 0 & 0.9514 & 0.9517 & 0.9479 & 0.9499 & 0.9479 & 0.9519 & 0.9485 \\
\hline & 0.1 & 0.9496 & 0.9496 & 0.9512 & 0.9463 & 0.9494 & 0.9505 & 0.9482 \\
\hline & 0.2 & 0.9521 & 0.9531 & 0.9465 & 0.9497 & 0.9504 & 0.9500 & 0.9476 \\
\hline & 0.3 & 0.9478 & 0.9537 & 0.9507 & 0.9519 & 0.9498 & 0.9501 & 0.9513 \\
\hline & 0.4 & 0.9490 & 0.9492 & 0.9499 & 0.9507 & 0.9513 & 0.9471 & 0.9483 \\
\hline & 0.5 & 0.9486 & 0.9519 & 0.9514 & 0.9492 & 0.9479 & 0.9502 & 0.9503 \\
\hline & 0.6 & 0.9495 & 0.9500 & 0.9493 & 0.9519 & 0.9520 & 0.9512 & 0.9500 \\
\hline & 0.7 & 0.9536 & 0.9517 & 0.9538 & 0.9520 & 0.9481 & 0.9512 & 0.9479 \\
\hline & 0.8 & 0.9530 & 0.9443 & 0.9509 & 0.9494 & 0.9513 & 0.9499 & 0.9508 \\
\hline & 0.9 & 0.9474 & 0.9478 & 0.9493 & 0.9479 & 0.9485 & 0.9516 & 0.9489 \\
\hline \multirow{19}{*}{ 은 } & -0.9 & 0.9522 & 0.9508 & 0.9489 & 0.9491 & 0.9488 & 0.9497 & 0.9501 \\
\hline & -0.8 & 0.9556 & 0.9510 & 0.9521 & 0.9521 & 0.9493 & 0.9504 & 0.9495 \\
\hline & -0.7 & 0.9462 & 0.9519 & 0.9514 & 0.9529 & 0.9511 & 0.9458 & 0.9476 \\
\hline & -0.6 & 0.9494 & 0.9510 & 0.9431 & 0.9470 & 0.9520 & 0.9461 & 0.9509 \\
\hline & -0.5 & 0.9511 & 0.9488 & 0.9487 & 0.9534 & 0.9503 & 0.9544 & 0.9456 \\
\hline & -0.4 & 0.9525 & 0.9459 & 0.9513 & 0.9517 & 0.9456 & 0.9519 & 0.9502 \\
\hline & -0.3 & 0.9506 & 0.9499 & 0.9493 & 0.9514 & 0.9492 & 0.9494 & 0.9511 \\
\hline & -0.2 & 0.9502 & 0.9530 & 0.9527 & 0.9516 & 0.9497 & 0.9456 & 0.9483 \\
\hline & -0.1 & 0.9495 & 0.9507 & 0.9500 & 0.9513 & 0.9515 & 0.9479 & 0.9487 \\
\hline & 0 & 0.9521 & 0.9511 & 0.9484 & 0.9504 & 0.9474 & 0.9519 & 0.9492 \\
\hline & 0.1 & 0.9492 & 0.9490 & 0.9501 & 0.9464 & 0.9497 & 0.9505 & 0.9475 \\
\hline & 0.2 & 0.9495 & 0.9518 & 0.9464 & 0.9500 & 0.9513 & 0.9509 & 0.9474 \\
\hline & 0.3 & 0.9487 & 0.9540 & 0.9510 & 0.9522 & 0.9490 & 0.9491 & 0.9516 \\
\hline & 0.4 & 0.9500 & 0.9483 & 0.9491 & 0.9510 & 0.9510 & 0.9473 & 0.9488 \\
\hline & 0.5 & 0.9498 & 0.9528 & 0.9517 & 0.9498 & 0.9474 & 0.9499 & 0.9507 \\
\hline & 0.6 & 0.9503 & 0.9505 & 0.9511 & 0.9531 & 0.9521 & 0.9509 & 0.9501 \\
\hline & 0.7 & 0.9556 & 0.9526 & 0.9522 & 0.9531 & 0.9483 & 0.9504 & 0.9483 \\
\hline & 0.8 & 0.9514 & 0.9455 & 0.9513 & 0.9498 & 0.9506 & 0.9500 & 0.9515 \\
\hline & 0.9 & 0.9513 & 0.9486 & 0.9490 & 0.9497 & 0.9496 & 0.9528 & 0.9484 \\
\hline
\end{tabular}



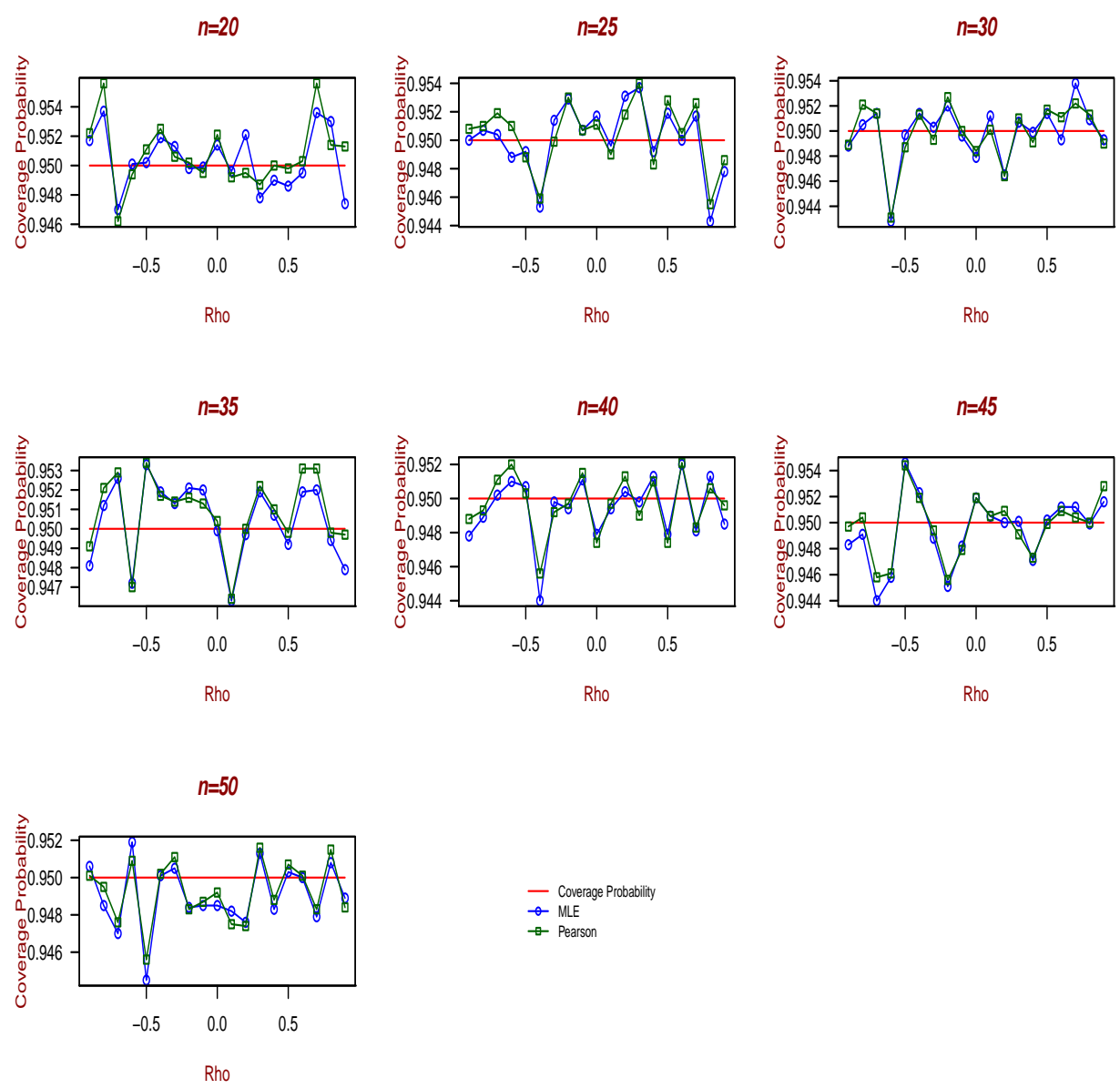

Figure 4.9: Comparison Coverage Probability Confidence Interval

It can be observed form Figure 4.9 that, in both cases (a) and (b), the coverage probabilities of the $95 \%$ confidence intervals of $\rho$ are close to 0.95 and with a small difference between them. 
Table 4.13: Comparison Average Width CI

\begin{tabular}{|c|c|c|c|c|c|c|c|c|}
\hline & \multirow{2}{*}{ Rho } & \multicolumn{7}{|c|}{ SAMPLE SIZE } \\
\hline & & 20 & 25 & 30 & 35 & 40 & 45 & 50 \\
\hline \multirow{19}{*}{ (0) } & -0.9 & 0.2049 & 0.1760 & 0.1574 & 0.1428 & 0.1310 & 0.1224 & 0.1145 \\
\hline & -0.8 & 0.3663 & 0.3162 & 0.2847 & 0.2591 & 0.2408 & 0.2253 & 0.2121 \\
\hline & -0.7 & 0.4889 & 0.4313 & 0.3884 & 0.3565 & 0.3331 & 0.3120 & 0.2953 \\
\hline & -0.6 & 0.5900 & 0.5209 & 0.4732 & 0.4375 & 0.4075 & 0.3826 & 0.3627 \\
\hline & -0.5 & 0.6671 & 0.5951 & 0.5422 & 0.5018 & 0.4681 & 0.4420 & 0.4179 \\
\hline & -0.4 & 0.7256 & 0.6511 & 0.5967 & 0.5524 & 0.5199 & 0.4892 & 0.4632 \\
\hline & -0.3 & 0.7713 & 0.6951 & 0.6371 & 0.5919 & 0.5548 & 0.5236 & 0.4977 \\
\hline & -0.2 & 0.8040 & 0.7248 & 0.6658 & 0.6189 & 0.5798 & 0.5488 & 0.5217 \\
\hline & -0.1 & 0.8219 & 0.7418 & 0.6821 & 0.6352 & 0.5963 & 0.5637 & 0.5365 \\
\hline & 0 & 0.8279 & 0.7486 & 0.6878 & 0.6401 & 0.6011 & 0.5688 & 0.5409 \\
\hline & 0.1 & 0.8214 & 0.7426 & 0.6825 & 0.6343 & 0.5963 & 0.5637 & 0.5360 \\
\hline & 0.2 & 0.8028 & 0.7256 & 0.6651 & 0.6185 & 0.5804 & 0.5495 & 0.5217 \\
\hline & 0.3 & 0.7728 & 0.6959 & 0.6370 & 0.5919 & 0.5541 & 0.5243 & 0.4978 \\
\hline & 0.4 & 0.7284 & 0.6517 & 0.5961 & 0.5523 & 0.5179 & 0.4879 & 0.4630 \\
\hline & 0.5 & 0.6673 & 0.5953 & 0.5418 & 0.5024 & 0.4685 & 0.4412 & 0.4181 \\
\hline & 0.6 & 0.5857 & 0.5238 & 0.4737 & 0.4359 & 0.4071 & 0.3835 & 0.3628 \\
\hline & 0.7 & 0.4893 & 0.4301 & 0.3897 & 0.3582 & 0.3320 & 0.3107 & 0.2938 \\
\hline & 0.8 & 0.3642 & 0.3167 & 0.2845 & 0.2596 & 0.2408 & 0.2246 & 0.2119 \\
\hline & 0.9 & 0.2063 & 0.1769 & 0.1572 & 0.1422 & 0.1308 & 0.1225 & 0.1147 \\
\hline \multirow{19}{*}{ e } & -0.9 & 0.2033 & 0.1747 & 0.1562 & 0.1419 & 0.1302 & 0.1218 & 0.1140 \\
\hline & -0.8 & 0.3667 & 0.3161 & 0.2843 & 0.2587 & 0.2403 & 0.2250 & 0.2118 \\
\hline & -0.7 & 0.4922 & 0.4332 & 0.3896 & 0.3572 & 0.3337 & 0.3125 & 0.2958 \\
\hline & -0.6 & 0.5971 & 0.5255 & 0.4763 & 0.4400 & 0.4093 & 0.3842 & 0.3640 \\
\hline & -0.5 & 0.6773 & 0.6022 & 0.5474 & 0.5058 & 0.4714 & 0.4447 & 0.4203 \\
\hline & -0.4 & 0.7388 & 0.6606 & 0.6037 & 0.5579 & 0.5245 & 0.4931 & 0.4664 \\
\hline & -0.3 & 0.7872 & 0.7064 & 0.6457 & 0.5988 & 0.5605 & 0.5283 & 0.5018 \\
\hline & -0.2 & 0.8218 & 0.7377 & 0.6757 & 0.6267 & 0.5863 & 0.5543 & 0.5265 \\
\hline & -0.1 & 0.8406 & 0.7556 & 0.6928 & 0.6437 & 0.6034 & 0.5696 & 0.5416 \\
\hline & 0 & 0.8470 & 0.7627 & 0.6987 & 0.6489 & 0.6083 & 0.5748 & 0.5460 \\
\hline & 0.1 & 0.8402 & 0.7563 & 0.6930 & 0.6429 & 0.6034 & 0.5696 & 0.5410 \\
\hline & 0.2 & 0.8204 & 0.7385 & 0.6749 & 0.6265 & 0.5869 & 0.5550 & 0.5264 \\
\hline & 0.3 & 0.7885 & 0.7073 & 0.6457 & 0.5988 & 0.5597 & 0.5290 & 0.5019 \\
\hline & 0.4 & 0.7419 & 0.6611 & 0.6031 & 0.5579 & 0.5225 & 0.4917 & 0.4664 \\
\hline & 0.5 & 0.6777 & 0.6024 & 0.5471 & 0.5065 & 0.4717 & 0.4439 & 0.4204 \\
\hline & 0.6 & 0.5922 & 0.5282 & 0.4768 & 0.4383 & 0.4091 & 0.3851 & 0.3641 \\
\hline & 0.7 & 0.4926 & 0.4322 & 0.3909 & 0.3591 & 0.3327 & 0.3111 & 0.2942 \\
\hline & 0.8 & 0.3645 & 0.3165 & 0.2843 & 0.2592 & 0.2405 & 0.2243 & 0.2115 \\
\hline & 0.9 & 0.2049 & 0.1756 & 0.1560 & 0.1413 & 0.1301 & 0.1219 & 0.1141 \\
\hline
\end{tabular}



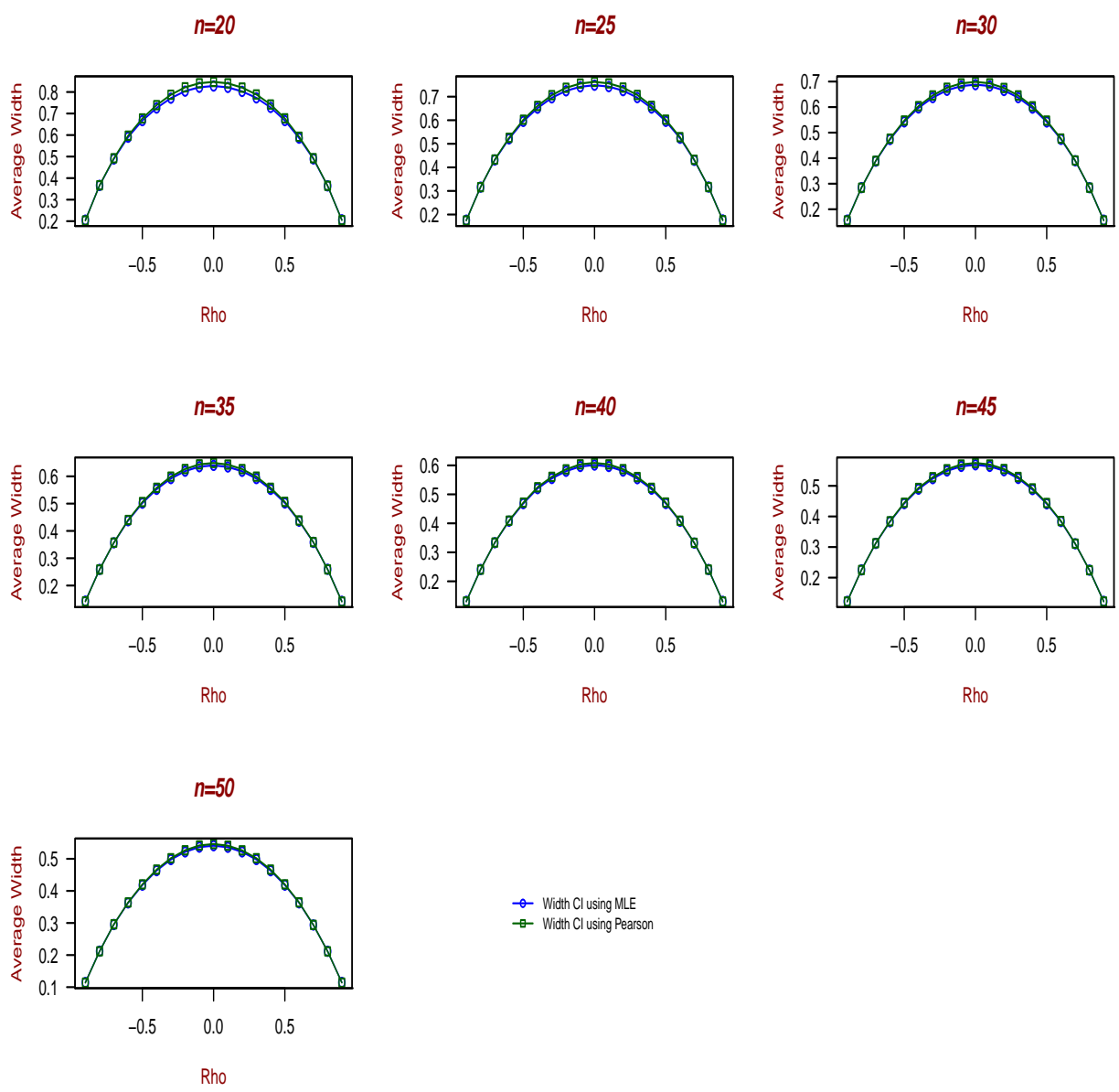

$\rightarrow$ Width Cl using MLE
+ Width Cl using Pearso

Figure 4.10: Comparison Average Width of the 95\% Confidence Interval

As presented in Figure 4.10, the average width of confidence intervals of $\rho$ is smaller when the MLE is used except for the extreme values of $\rho$.

In conclusion, it is better to use the MLE than the Pearson Correlation Coefficient if we want to find confidence intervals to estimate the true value of $\rho$. 


\subsection{Testing hypothesis}

A continuation we compare the probability of type I error of testing

$$
H_{0}: \rho=\rho_{0} \quad \text { vs. } H a: \rho \neq \rho_{0}
$$

and power of testing

$$
H_{0}: \rho=\rho_{0} \quad \text { vs. } \quad H a: \rho \neq \rho_{0}
$$

where $\rho_{0}$ is the true value of $\rho$ using both estimators mentioned before: (a) the MLE and (b) the Pearson Correlation Coefficient.

\subsubsection{Probability of a Type I error}

In Table 4.14 the probability of type I error is presented when testing the hypothesis defined in (5.5). The values of $\rho$ were chosen to be from -0.9 to 0.9 in 0.1 increments and it was used $\alpha=0.05$ as the level of significance. In Figure 4.11 is evident that the type I errors for both (a) and (b) are close to 0.05 
Table 4.14: Comparison Type I Error

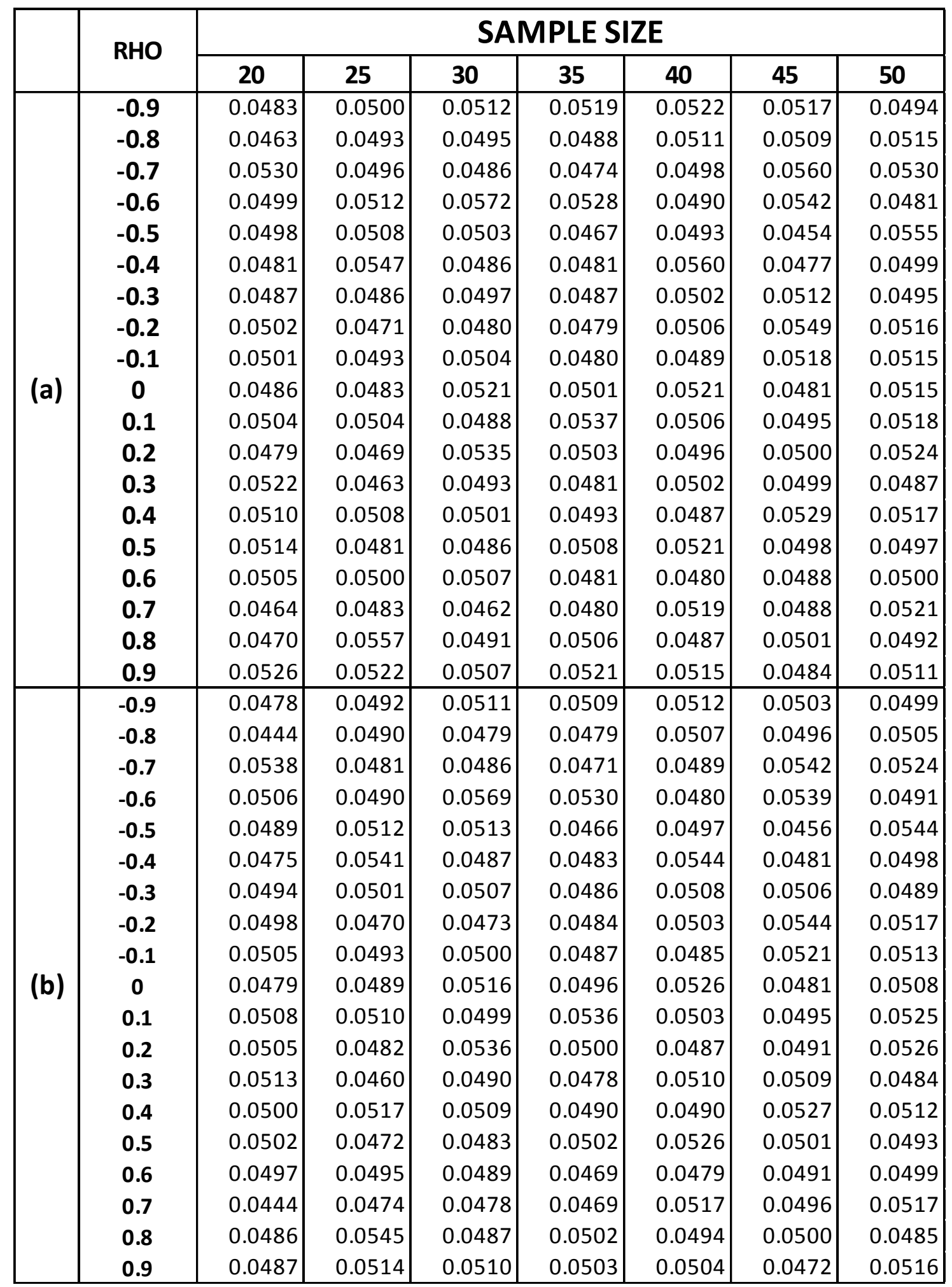



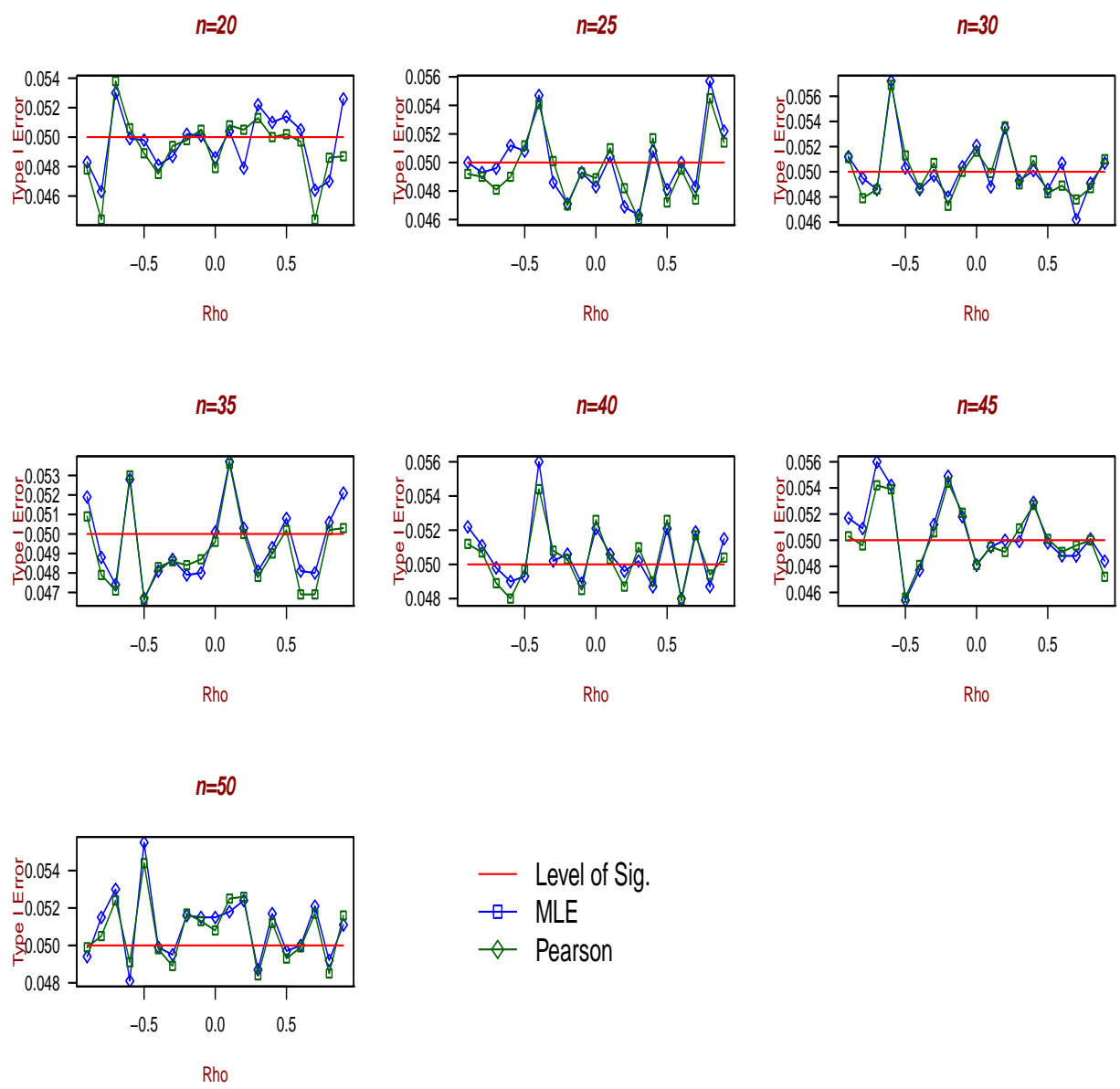

$$
\begin{aligned}
& - \text { Level of Sig. } \\
& \square \text { MLE } \\
& \neg \text { Pearson }
\end{aligned}
$$

Figure 4.11: Comparison Type I error

\subsubsection{Power of the Test}

In order to calculate the power of testing the hypothesis in (4.8), different simulations were conducted.

Assuming $\rho=\rho_{0}$ take the values from -0.8 to 0.8 in 0.1 increments that were use in the null hypothesis, the values for $\rho_{1}$ in the alternative hypothesis were chosen as $\rho_{1}=\rho \pm 0.10$. Table 4.15 presents the values of the power using the MLE and Table 4.16 using the Pearson Correlation Coefficient. 
Table 4.15: Power MLE when $H a: \rho_{1}=\rho \pm 0.10$

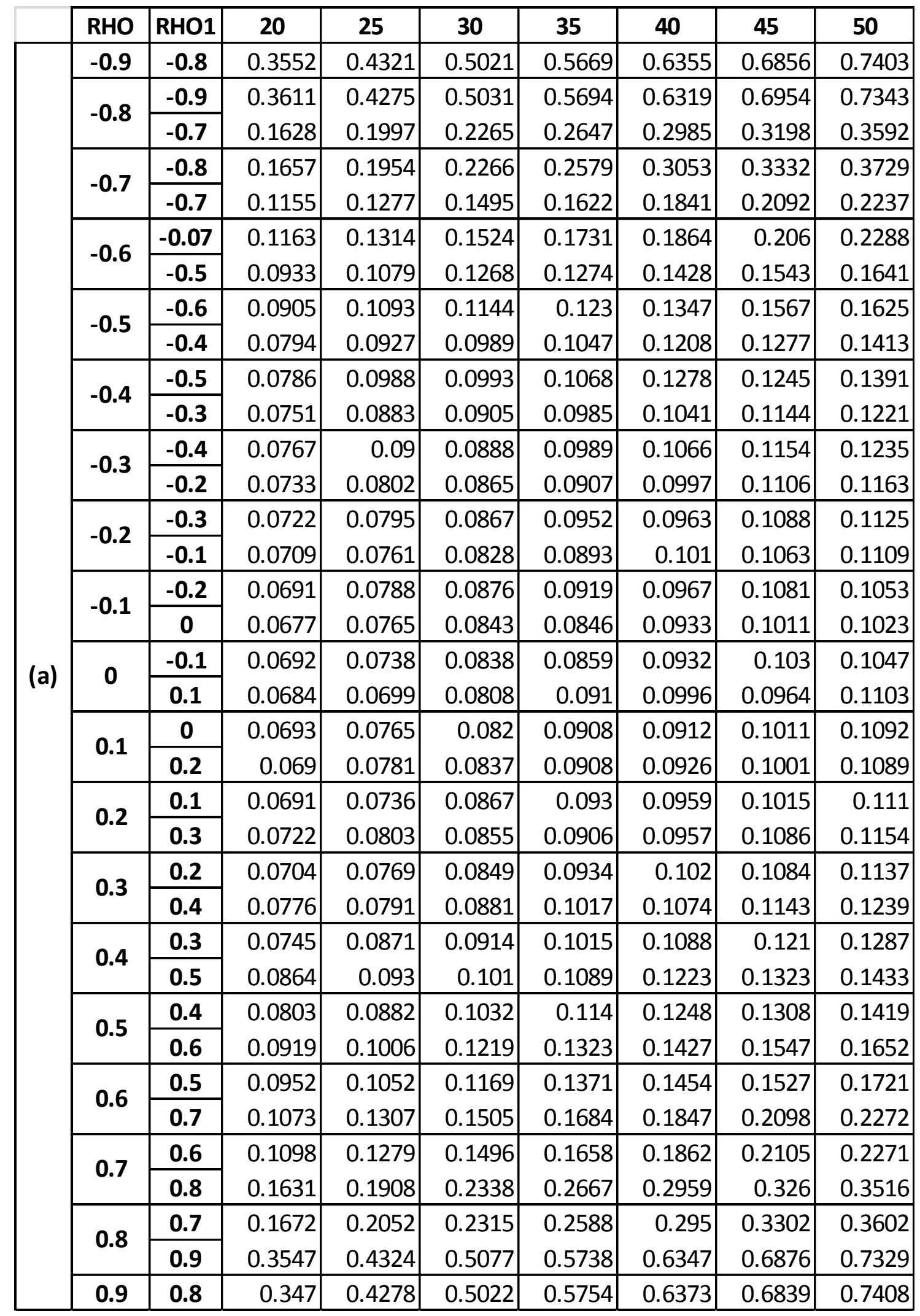


Table 4.16: Power Pearson when $H a: \rho_{1}=\rho \pm .10$

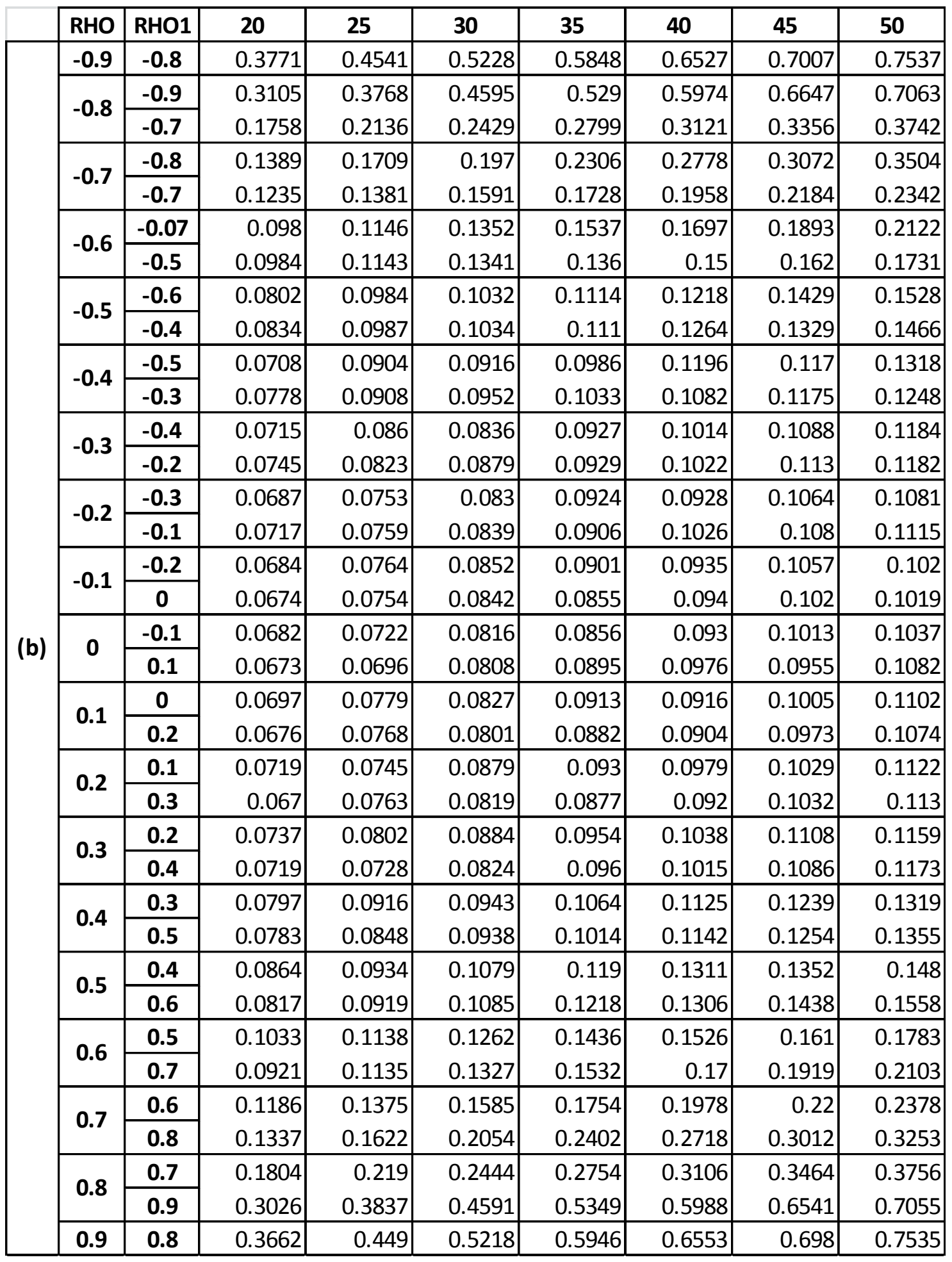



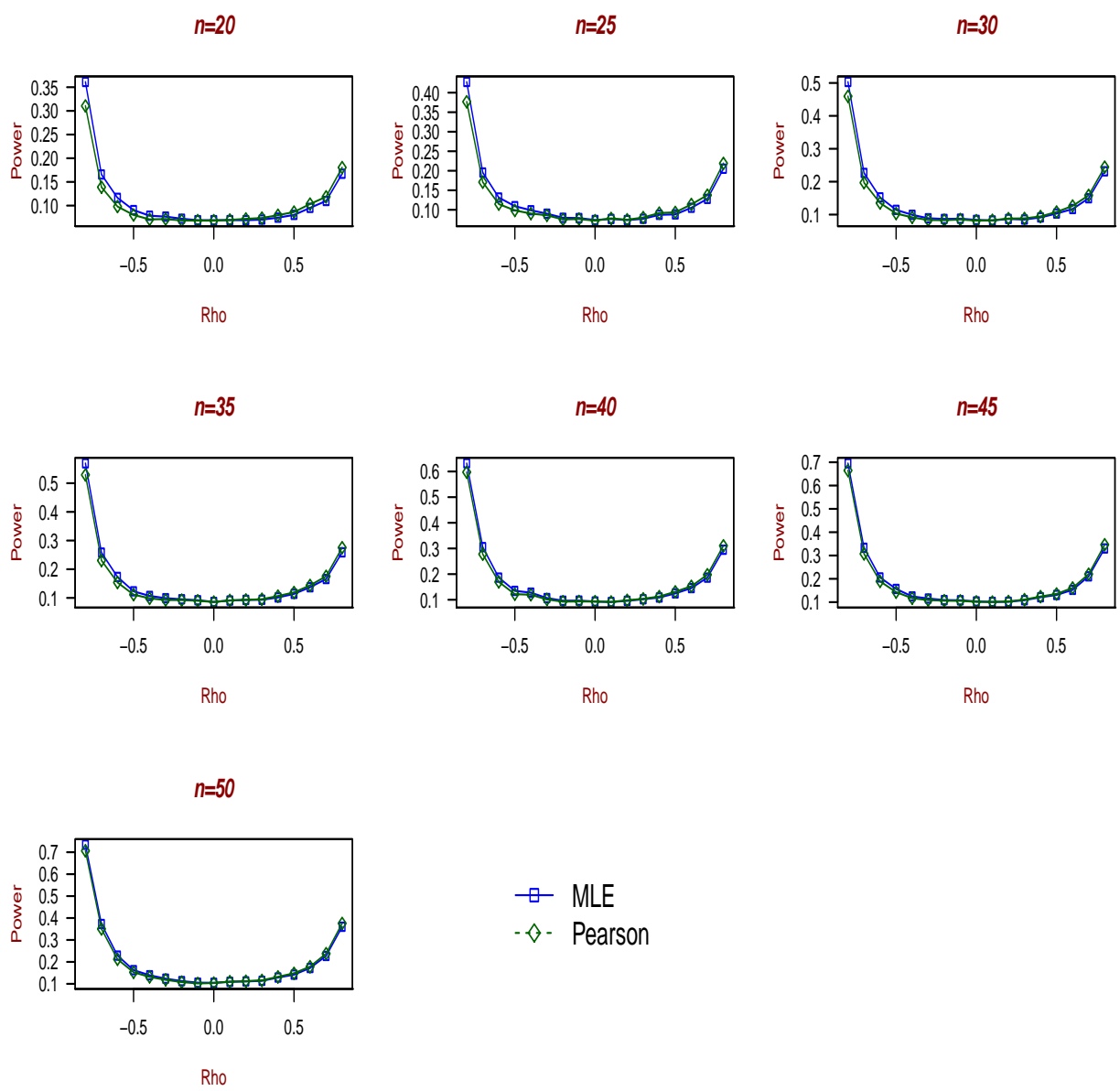

$\square$ MLE

- $\diamond$ - Pearson

Figure 4.12: Comparison Power Ha: $\rho=\rho-0.10$ 

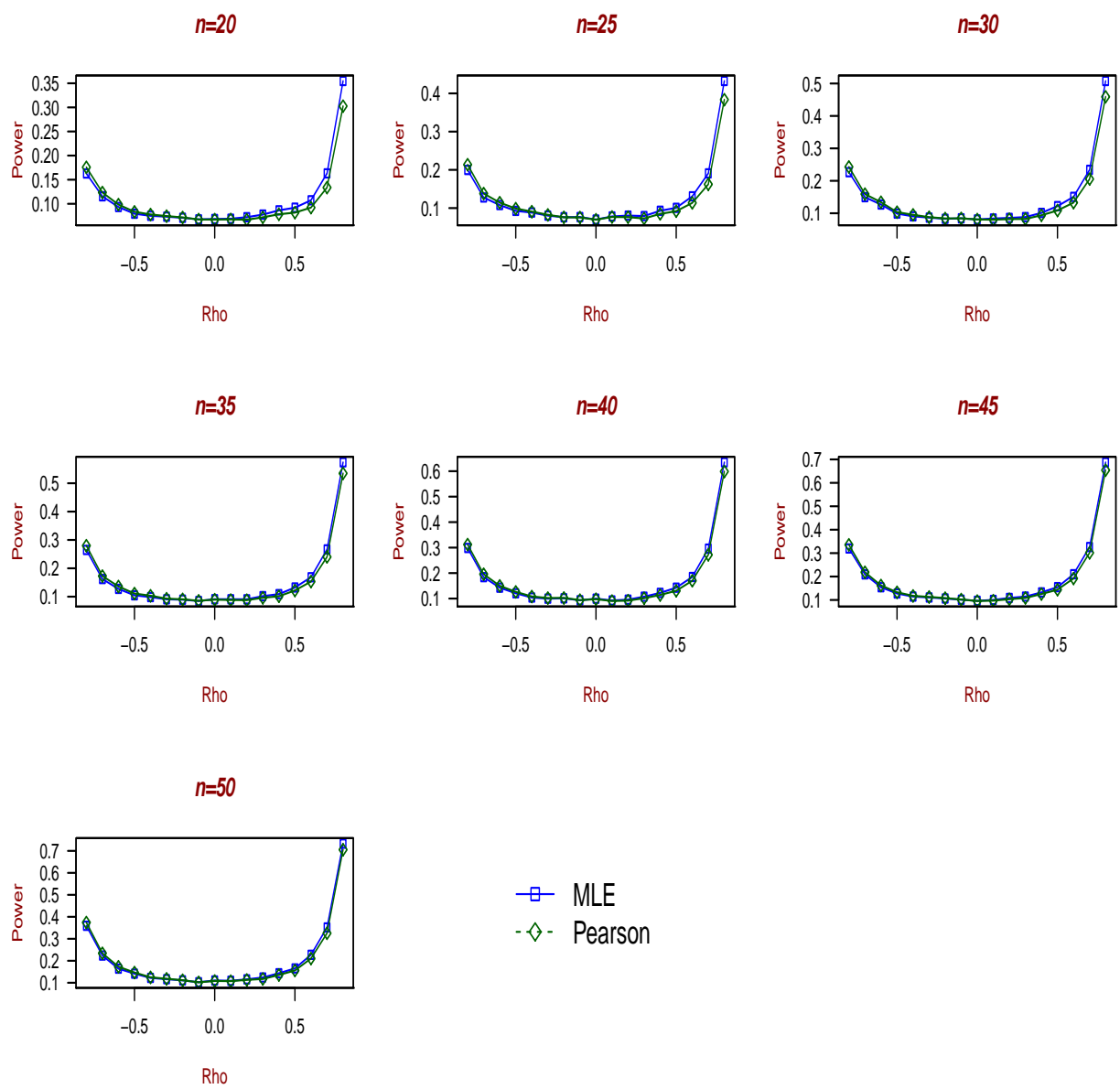

$$
\begin{aligned}
& \rightarrow \text { MLE } \\
& -\diamond-\text { Pearson }
\end{aligned}
$$

Figure 4.13: Comparison Power $\mathrm{Ha}: \rho=\rho+0.10$

It can be observed in Figures 4.12 and 4.13 the power of testing for the MLE and Pearson Correlation Coefficient. There is no difference between them. Moreover, the power of testing is much higher when $\rho$ approches to its extreme values.

Additionally, $\rho_{1}=\rho-0.05$ and $\rho_{1}=\rho+0.05$ were also evaluated for $\rho=\rho_{0}$ ranging from -0.9 to 0.9 . Tables 4.17 and 4.18 present the power of testing when using the (a) MLE and (b) the Pearson Correlation Coefficient, respectively and can be observed in Figures 4.14 and 4.15 . 
Table 4.17: Power MLE when $H a: \rho_{1}=\rho \pm .05$

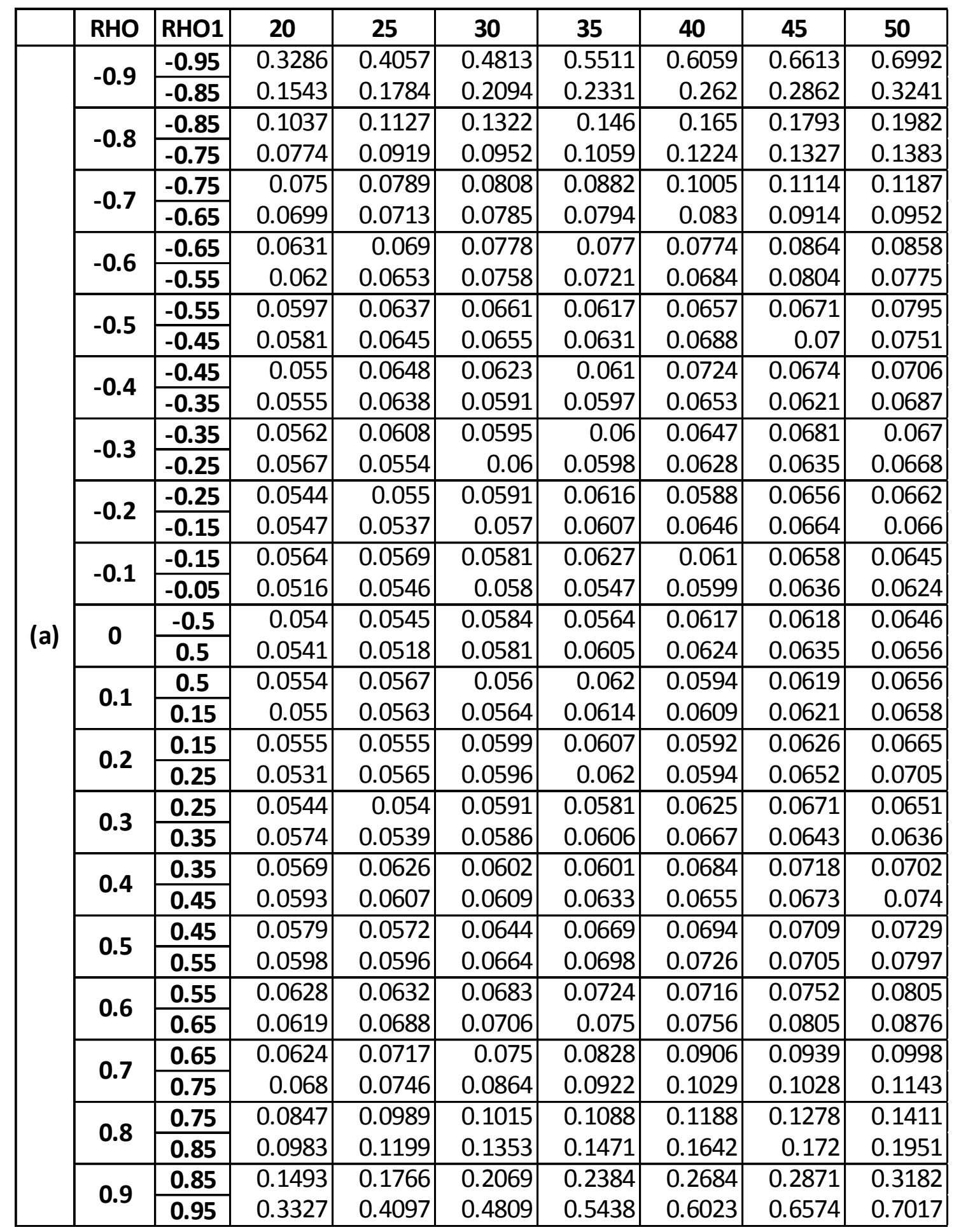


Table 4.18: Power Pearson when $H a: \rho_{1}=\rho \pm .05$

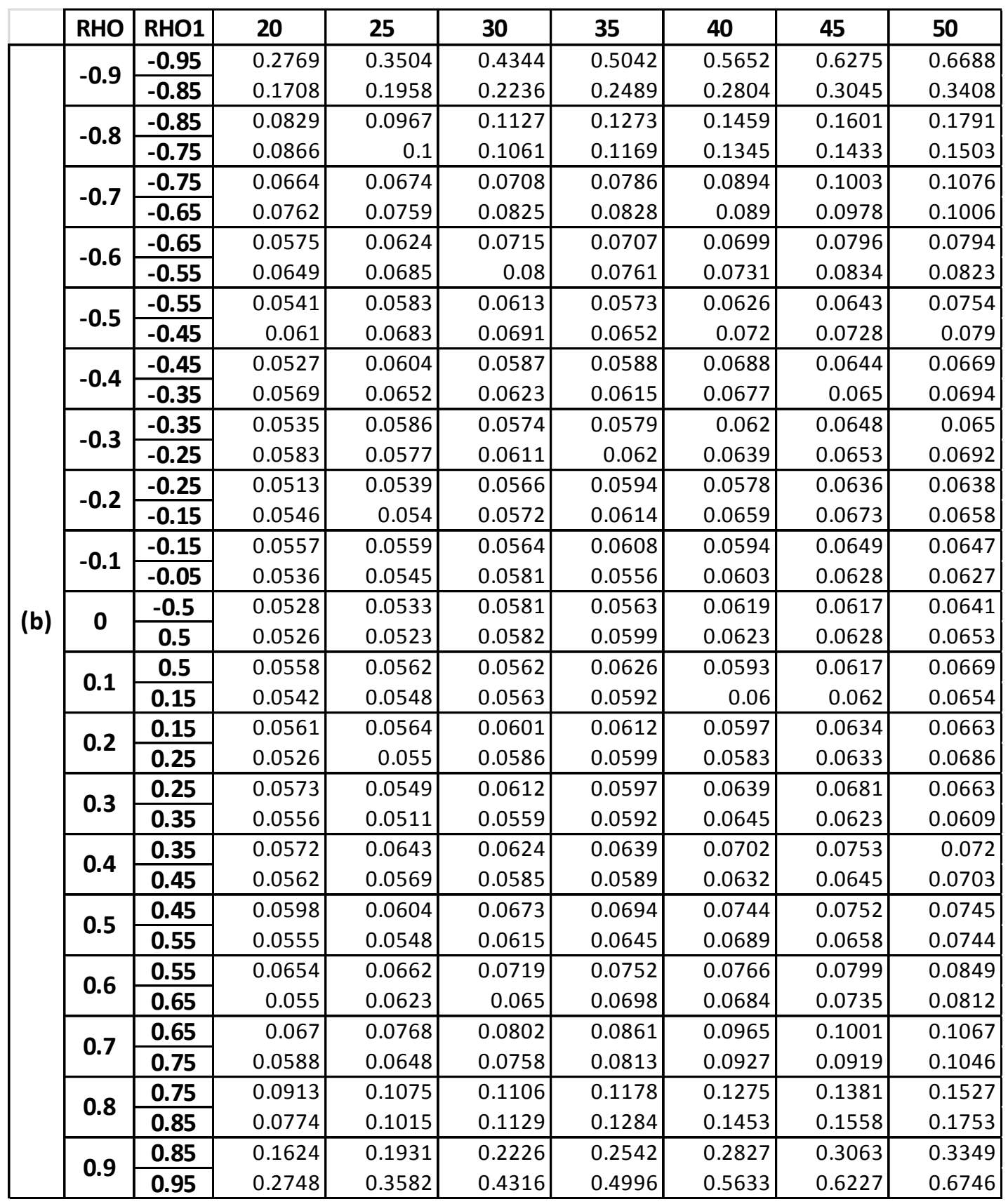



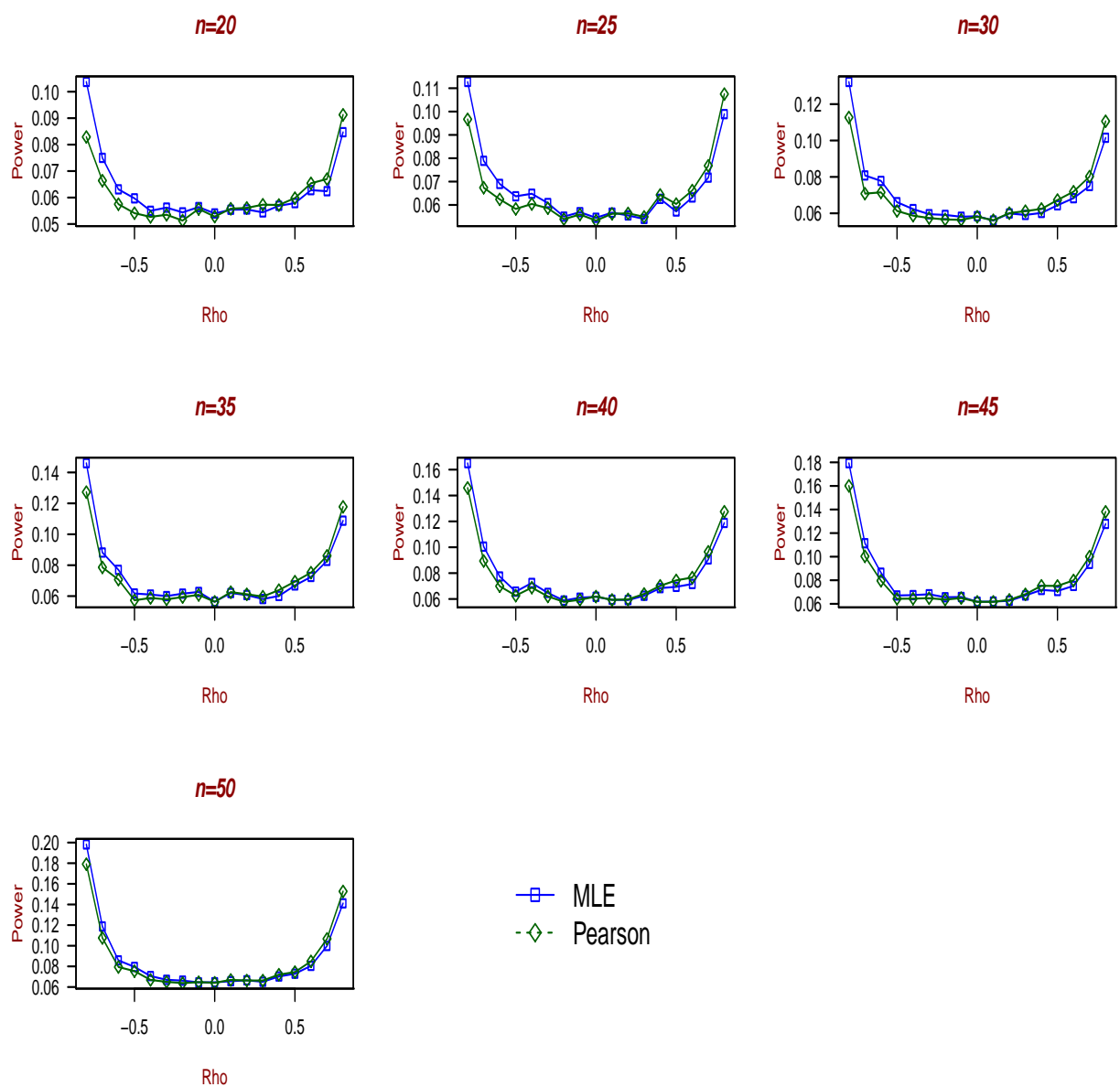

$\square$ MLE

- $\diamond$ - Pearson

Figure 4.14: Comparison Power Ha: $\rho=\rho-0.05$ 

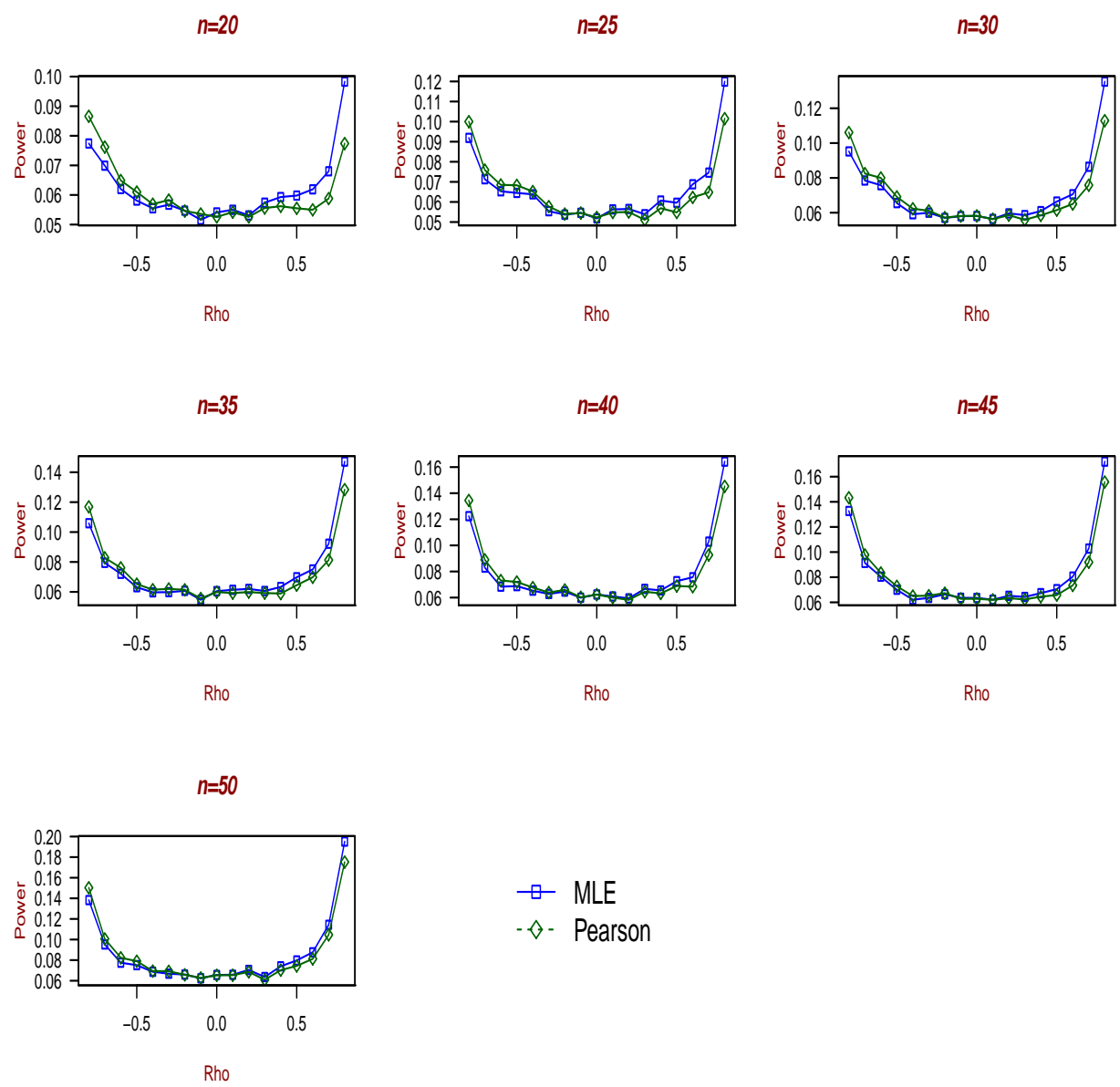

$$
\begin{aligned}
& \rightarrow-\text { MLE } \\
& -\diamond-\text { Pearson }
\end{aligned}
$$

Figure 4.15: Comparison Power $\mathrm{Ha}: \rho=\rho+0.05$

It can be observed in Figures 4.14 and 4.15 the power of testing for the MLE and Pearson Correlation Coefficient. Like before, there is no difference when using both estimators, and the power of testing is much higher when $\rho$ approches to its extreme values. 


\section{CHAPTER 5 \\ NUMERICAL STUDY ON THE CASE OF TRIVARIATE NORMAL DISTRIBUTION}

In Chapter 3 was found the MLE of the correlation coefficient in the case of equal variances in the Trivariate Normal Distribution. In this chapter simulations are made to evaluate its performance to estimate the true value of $\rho$.

For our simulations, we assumed $\mu^{T}=(0,0,0)$ and $\sigma^{2}=4$ and picked seven different sample sizes $(n)$, with $n$ ranging from 20 to 50 in increments of 5 and the 14 values $\rho$ that ranges from -0.4 to 0.9 in 0.1 increments. One thousand of replicates were run.

The Chapter 5 is organized as follows: In Section 5.1 are discussed three estimators of $\rho$, and the MSE and Bias have been evaluated. Confidence intervals for the correlation coefficient are calculated in Section 5.2 and the probability of type I error and power when testing some hypotheses are found in Section 5.3.

\subsection{Comparison Estimators of $\rho$}

Three estimators of the correlation coefficient $\rho$ have been considered: (a) MLE defined in Expression (3.33), (b) The Average Pearson and (c) the Average MLE. The Average Pearson and Average of the MLE's are found computing the average of the three pairwise Pearson correlation coefficients and Bivariate MLE of $\rho$, respectively, discussed in Chapter 2. 


\subsubsection{Point Estimators}

\begin{tabular}{|c|c|c|c|c|c|c|c|c|}
\hline & \multirow{2}{*}{ Rho } & \multicolumn{7}{|c|}{ SAMPLE SIZE } \\
\hline & & 20 & 25 & 30 & 35 & 40 & 45 & 50 \\
\hline \multirow{14}{*}{$\tilde{\sigma}$} & -0.4 & -0.3955 & -0.3969 & $\begin{array}{l}-0.3974 \\
\end{array}$ & -0.3981 & -0.3984 & -0.3983 & -0.3981 \\
\hline & -0.3 & -0.2945 & -0.2957 & -0.2952 & -0.2971 & -0.2965 & -0.2974 & -0.2976 \\
\hline & -0.2 & -0.1967 & -0.1961 & -0.1958 & -0.1968 & -0.1972 & -0.1994 & -0.1975 \\
\hline & -0.1 & -0.0958 & -0.0967 & -0.0993 & -0.0991 & -0.0982 & -0.0989 & -0.0989 \\
\hline & 0 & 0.0004 & 0.0007 & -0.0017 & 0.0005 & 0.0002 & -0.0001 & 0.0004 \\
\hline & 0.1 & 0.0983 & 0.0967 & 0.1001 & 0.0995 & 0.0992 & 0.0979 & 0.0998 \\
\hline & 0.2 & 0.1935 & 0.1937 & 0.1947 & 0.1961 & 0.1956 & 0.1967 & 0.1964 \\
\hline & 0.3 & 0.2903 & 0.2888 & 0.2922 & 0.2937 & 0.2946 & 0.2941 & 0.2954 \\
\hline & 0.4 & 0.3848 & 0.3873 & 0.3904 & 0.3937 & 0.3940 & 0.3941 & 0.3935 \\
\hline & 0.5 & 0.4826 & 0.4866 & 0.4886 & 0.4900 & 0.4915 & 0.4935 & 0.4943 \\
\hline & 0.6 & 0.5806 & 0.5864 & 0.5875 & 0.5904 & 0.5907 & 0.5921 & 0.5930 \\
\hline & 0.7 & 0.6820 & 0.6845 & 0.6879 & 0.6896 & 0.6906 & 0.6928 & 0.6923 \\
\hline & 0.8 & 0.7848 & 0.7884 & 0.7901 & 0.7912 & 0.7932 & 0.7934 & 0.7941 \\
\hline & 0.9 & 0.8905 & 0.8923 & 0.8940 & 0.8949 & 0.8957 & 0.8960 & 0.8963 \\
\hline \multirow{14}{*}{$\tilde{2}$} & -0.4 & -0.3905 & -0.3931 & -0.3942 & -0.3953 & -0.3960 & -0.3961 & -0.3962 \\
\hline & -0.3 & -0.2927 & -0.2944 & -0.2942 & -0.2962 & -0.2958 & -0.2968 & -0.2970 \\
\hline & -0.2 & -0.1966 & -0.1962 & -0.1958 & -0.1968 & -0.1972 & -0.1994 & -0.1975 \\
\hline & -0.1 & -0.0963 & -0.0969 & -0.0996 & -0.0993 & -0.0983 & -0.0990 & -0.0992 \\
\hline & 0 & 0.0004 & 0.0006 & -0.0019 & 0.0004 & 0.0001 & -0.0001 & 0.0004 \\
\hline & 0.1 & 0.0993 & 0.0977 & 0.1008 & 0.1000 & 0.0998 & 0.0984 & 0.1001 \\
\hline & 0.2 & 0.1959 & 0.1957 & 0.1963 & 0.1977 & 0.1969 & 0.1979 & 0.1975 \\
\hline & 0.3 & 0.2944 & 0.2922 & 0.2951 & 0.2962 & 0.2968 & 0.2961 & 0.2971 \\
\hline & 0.4 & 0.3905 & 0.3919 & 0.3944 & 0.3971 & 0.3968 & 0.3967 & 0.3958 \\
\hline & 0.5 & 0.4898 & 0.4923 & 0.4934 & 0.4941 & 0.4953 & 0.4968 & 0.4972 \\
\hline & 0.6 & 0.5885 & 0.5930 & 0.5930 & 0.5951 & 0.5947 & 0.5956 & 0.5963 \\
\hline & 0.7 & 0.6902 & 0.6910 & 0.6932 & 0.6941 & 0.6947 & 0.6965 & 0.6955 \\
\hline & 0.8 & 0.7920 & 0.7940 & 0.7946 & 0.7951 & 0.7966 & 0.7965 & 0.7968 \\
\hline & 0.9 & 0.8952 & 0.8960 & 0.8970 & 0.8974 & 0.8979 & 0.8979 & 0.8980 \\
\hline \multirow{14}{*}{$\widetilde{u}$} & -0.4 & -0.3822 & -0.3865 & -0.3886 & -0.3905 & -0.3918 & -0.3924 & -0.3928 \\
\hline & -0.3 & -0.2860 & -0.2890 & -0.2898 & -0.2924 & -0.2924 & -0.2938 & -0.2944 \\
\hline & -0.2 & -0.1919 & -0.1925 & -0.1926 & -0.1941 & -0.1948 & -0.1973 & -0.1956 \\
\hline & -0.1 & -0.0939 & -0.0950 & -0.0980 & -0.0979 & -0.0971 & -0.0980 & -0.0982 \\
\hline & 0 & 0.0004 & 0.0006 & -0.0018 & 0.0004 & 0.0001 & -0.0001 & 0.0004 \\
\hline & 0.1 & 0.0969 & 0.0957 & 0.0991 & 0.0986 & 0.0985 & 0.0973 & 0.0991 \\
\hline & 0.2 & 0.1912 & 0.1920 & 0.1932 & 0.1950 & 0.1945 & 0.1957 & 0.1956 \\
\hline & 0.3 & 0.2878 & 0.2869 & 0.2905 & 0.2923 & 0.2935 & 0.2931 & 0.2943 \\
\hline & 0.4 & 0.3823 & 0.3853 & 0.3890 & 0.3923 & 0.3927 & 0.3930 & 0.3925 \\
\hline & 0.5 & 0.4803 & 0.4848 & 0.4871 & 0.4887 & 0.4905 & 0.4925 & 0.4935 \\
\hline & 0.6 & 0.5788 & 0.5850 & 0.5864 & 0.5895 & 0.5898 & 0.5913 & 0.5923 \\
\hline & 0.7 & 0.6808 & 0.6835 & 0.6871 & 0.6889 & 0.6901 & 0.6923 & 0.6919 \\
\hline & 0.8 & 0.7841 & 0.7879 & 0.7897 & 0.7909 & 0.7929 & 0.7932 & 0.7939 \\
\hline & 0.9 & 0.8903 & 0.8922 & 0.8939 & 0.8948 & 0.8956 & 0.8959 & 0.8962 \\
\hline
\end{tabular}

Table 5.1: Comparison Point Estimators Trivariate 

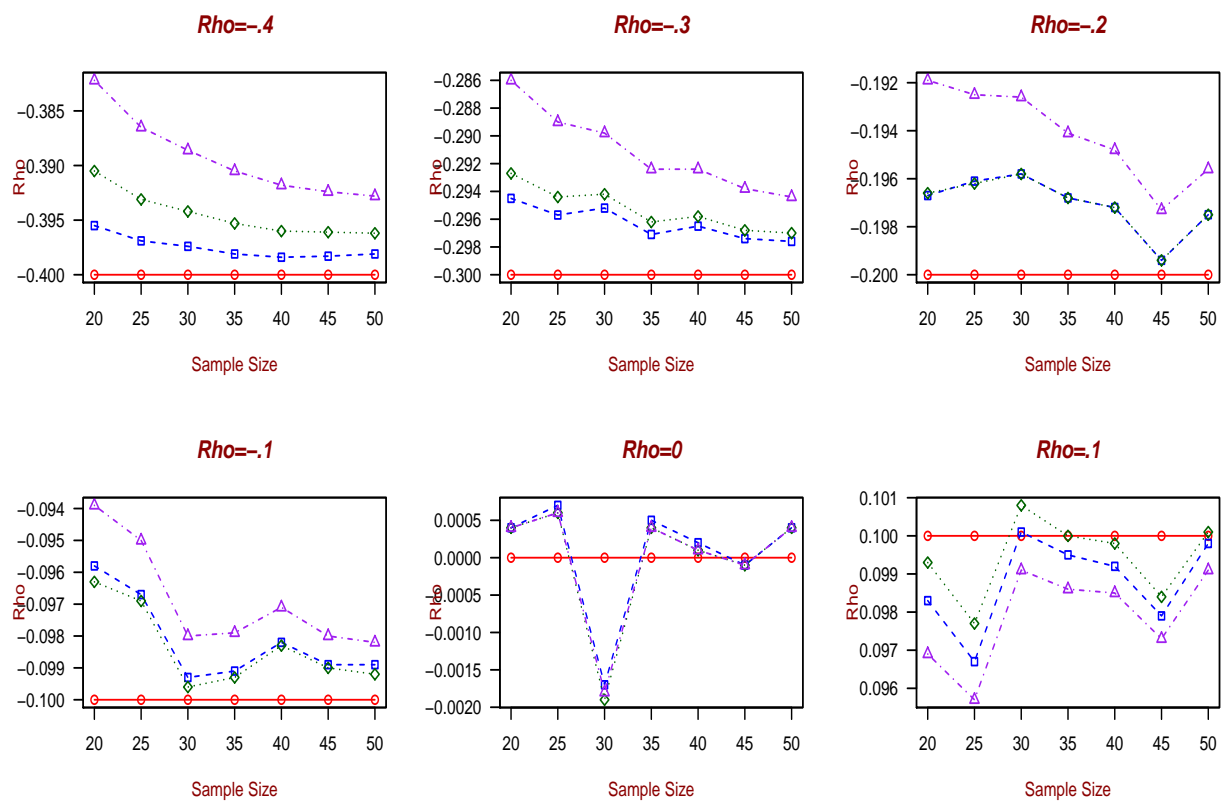

Rho $=.2$

Rho $=0.3$
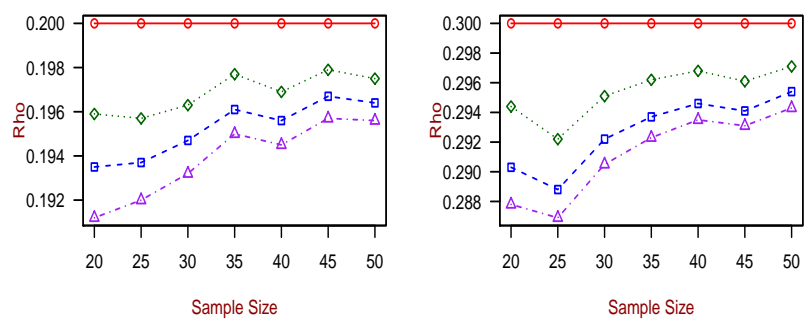

Rho=.4

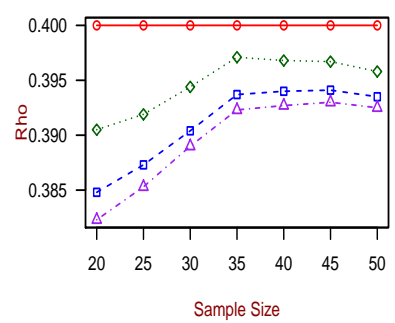

Rho=. 5

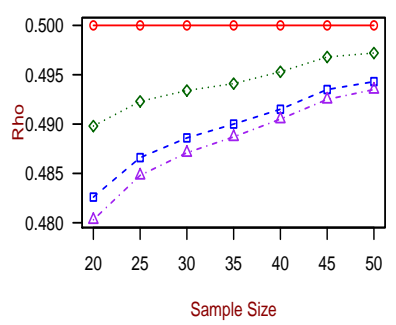

Rho=. 6
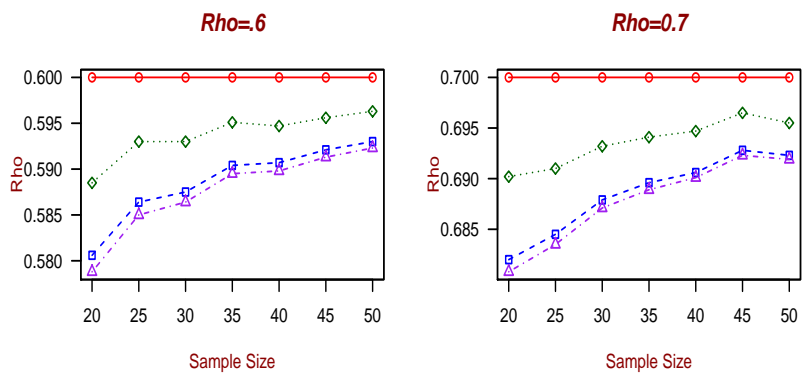

Rho $=.8$

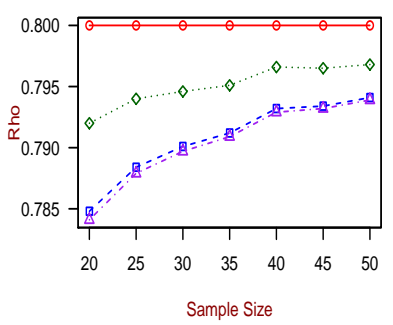

Rho=.9

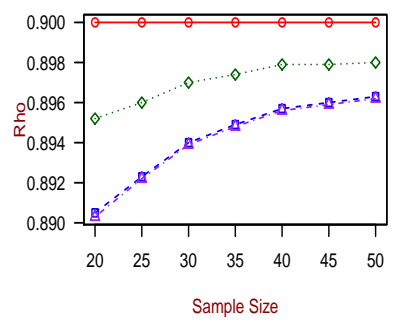

o- Rho

- - - MLE

$\diamond$ Av.Pearson

-A - Av.MLE

Figure 5.1: Comparison Point Estimators Trivariate Normal Distribution 
In Figure 5.1 the three point estimators are presented. These tree estimators are close to the true value of $\rho$, however, the average of Pearsons is closer to it, followed by the MLE. Notice also that when $\rho=0$, the three point estimators are the same.

\subsubsection{Study on the performance of the estimators}

In Table 5.2 is presented the Bias of the estimators and it have been visualized in Figure 5.2. It can be seen that the Average Pearson estimator has smaller bias except when $\rho=0$.

The mean square error MSE is presented in Figure 5.3 and corresponding table,

Table 5.3. The MSE for the tree estimators is small, but a difference between the Average Pearson and MLE can not be observable. 


\begin{tabular}{|c|c|c|c|c|c|c|c|c|}
\hline & \multirow{2}{*}{ Rho } & \multicolumn{7}{|c|}{ SAMPLE SIZE } \\
\hline & & 20 & 25 & 30 & 35 & 40 & 45 & 50 \\
\hline \multirow{14}{*}{$\tilde{\sigma}$} & -0.4 & -0.0045 & -0.0031 & -0.0026 & -0.0019 & -0.0016 & -0.0017 & -0.0019 \\
\hline & -0.3 & -0.0055 & -0.0043 & -0.0048 & -0.0029 & -0.0035 & -0.0026 & -0.0024 \\
\hline & -0.2 & -0.0033 & -0.0039 & -0.0042 & -0.0032 & -0.0028 & -0.0006 & -0.0025 \\
\hline & -0.1 & -0.0042 & -0.0033 & -0.0007 & -0.0009 & -0.0018 & -0.0011 & -0.0011 \\
\hline & 0 & -0.0004 & -0.0007 & 0.0017 & -0.0005 & -0.0002 & 0.0001 & -0.0004 \\
\hline & 0.1 & 0.0017 & 0.0033 & -0.0001 & 0.0005 & 0.0008 & 0.0021 & 0.0002 \\
\hline & 0.2 & 0.0065 & 0.0063 & 0.0053 & 0.0039 & 0.0044 & 0.0033 & 0.0036 \\
\hline & 0.3 & 0.0097 & 0.0112 & 0.0078 & 0.0063 & 0.0054 & 0.0059 & 0.0046 \\
\hline & 0.4 & 0.0152 & 0.0127 & 0.0096 & 0.0063 & 0.0060 & 0.0059 & 0.0065 \\
\hline & 0.5 & 0.0174 & 0.0134 & 0.0114 & 0.0100 & 0.0085 & 0.0065 & 0.0057 \\
\hline & 0.6 & 0.0194 & 0.0136 & 0.0125 & 0.0096 & 0.0093 & 0.0079 & 0.0070 \\
\hline & 0.7 & 0.0180 & 0.0155 & 0.0121 & 0.0104 & 0.0094 & 0.0072 & 0.0077 \\
\hline & 0.8 & 0.0152 & 0.0116 & 0.0099 & 0.0088 & 0.0068 & 0.0066 & 0.0059 \\
\hline & 0.9 & 0.0095 & 0.0077 & 0.0060 & 0.0051 & 0.0043 & 0.0040 & 0.0037 \\
\hline \multirow{14}{*}{$\underline{2}$} & -0.4 & -0.0095 & -0.0069 & -0.0058 & -0.0047 & -0.0040 & -0.0039 & -0.0038 \\
\hline & -0.3 & -0.0073 & -0.0056 & -0.0058 & -0.0038 & -0.0042 & -0.0032 & -0.0030 \\
\hline & -0.2 & -0.0034 & -0.0038 & -0.0042 & -0.0032 & -0.0028 & -0.0006 & -0.0025 \\
\hline & -0.1 & -0.0037 & -0.0031 & -0.0004 & -0.0007 & -0.0017 & -0.0010 & -0.0008 \\
\hline & 0 & -0.0004 & -0.0006 & 0.0019 & -0.0004 & -0.0001 & 0.0001 & -0.0004 \\
\hline & 0.1 & 0.0007 & 0.0023 & -0.0008 & 0.0000 & 0.0002 & 0.0016 & -0.0001 \\
\hline & 0.2 & 0.0041 & 0.0043 & 0.0037 & 0.0023 & 0.0031 & 0.0021 & 0.0025 \\
\hline & 0.3 & 0.0056 & 0.0078 & 0.0049 & 0.0038 & 0.0032 & 0.0039 & 0.0029 \\
\hline & 0.4 & 0.0095 & 0.0081 & 0.0056 & 0.0029 & 0.0032 & 0.0033 & 0.0042 \\
\hline & 0.5 & 0.0102 & 0.0077 & 0.0066 & 0.0059 & 0.0047 & 0.0032 & 0.0028 \\
\hline & 0.6 & 0.0115 & 0.0070 & 0.0070 & 0.0049 & 0.0053 & 0.0044 & 0.0037 \\
\hline & 0.7 & 0.0098 & 0.0090 & 0.0068 & 0.0059 & 0.0053 & 0.0035 & 0.0045 \\
\hline & 0.8 & 0.0080 & 0.0060 & 0.0054 & 0.0049 & 0.0034 & 0.0035 & 0.0032 \\
\hline & 0.9 & 0.0048 & 0.0040 & 0.0030 & 0.0026 & 0.0021 & 0.0021 & 0.0020 \\
\hline \multirow{14}{*}{$\tilde{u}$} & -0.4 & -0.0178 & -0.0135 & -0.0114 & -0.0095 & -0.0082 & -0.0076 & -0.0072 \\
\hline & -0.3 & -0.0140 & -0.0110 & -0.0102 & -0.0076 & -0.0076 & -0.0062 & -0.0056 \\
\hline & -0.2 & -0.0081 & -0.0075 & -0.0074 & -0.0059 & -0.0052 & -0.0027 & -0.0044 \\
\hline & -0.1 & -0.0061 & -0.0050 & -0.0020 & -0.0021 & -0.0029 & -0.0020 & -0.0018 \\
\hline & 0 & -0.0004 & -0.0006 & 0.0018 & -0.0004 & -0.0001 & 0.0001 & -0.0004 \\
\hline & 0.1 & 0.0031 & 0.0043 & 0.0009 & 0.0014 & 0.0015 & 0.0027 & 0.0009 \\
\hline & 0.2 & 0.0088 & 0.0080 & 0.0068 & 0.0050 & 0.0055 & 0.0043 & 0.0044 \\
\hline & 0.3 & 0.0122 & 0.0131 & 0.0095 & 0.0077 & 0.0065 & 0.0069 & 0.0057 \\
\hline & 0.4 & 0.0177 & 0.0147 & 0.0110 & 0.0077 & 0.0073 & 0.0070 & 0.0075 \\
\hline & 0.5 & 0.0197 & 0.0152 & 0.0129 & 0.0113 & 0.0095 & 0.0075 & 0.0065 \\
\hline & 0.6 & 0.0212 & 0.0150 & 0.0136 & 0.0105 & 0.0102 & 0.0087 & 0.0077 \\
\hline & 0.7 & 0.0192 & 0.0165 & 0.0129 & 0.0111 & 0.0099 & 0.0077 & 0.0081 \\
\hline & 0.8 & 0.0159 & 0.0121 & 0.0103 & 0.0091 & 0.0071 & 0.0068 & 0.0061 \\
\hline & 0.9 & 0.0097 & 0.0078 & 0.0061 & 0.0052 & 0.0044 & 0.0041 & 0.0038 \\
\hline
\end{tabular}

Table 5.2: Comparison Bias of Estimators Trivariate 

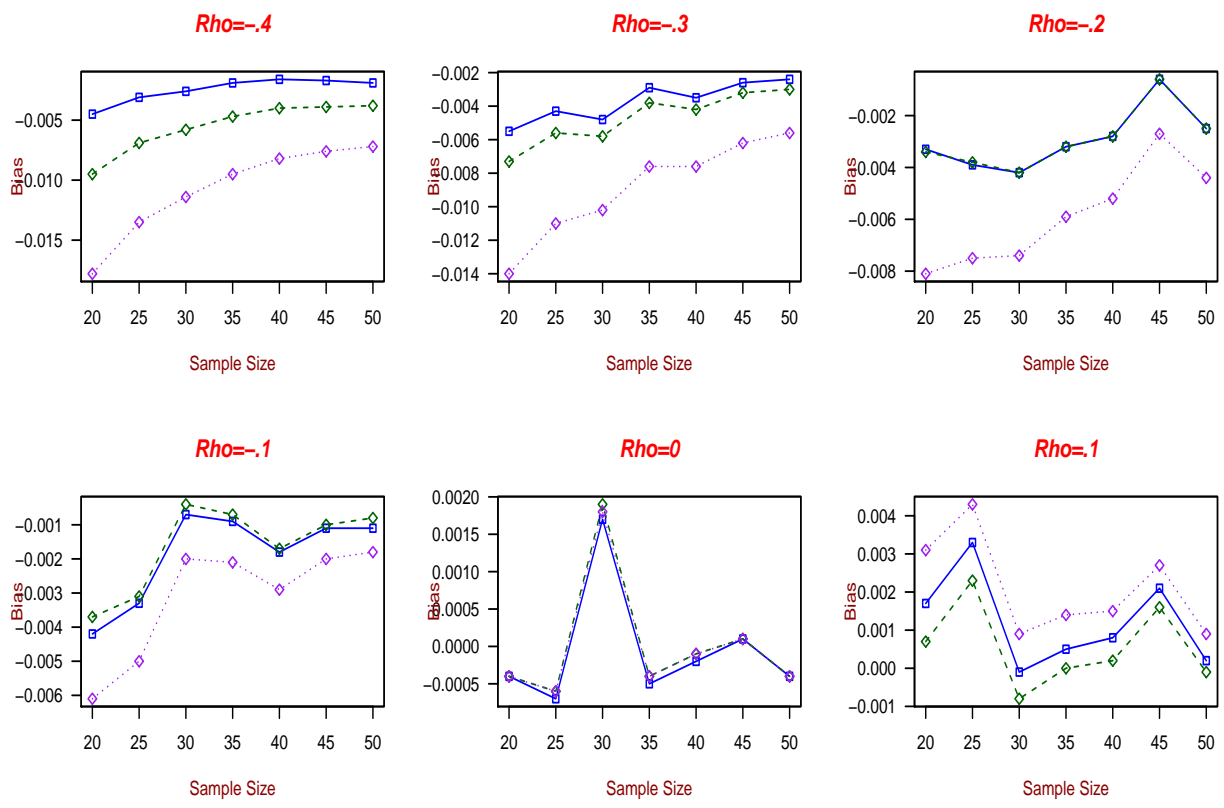

Rho $=.2$
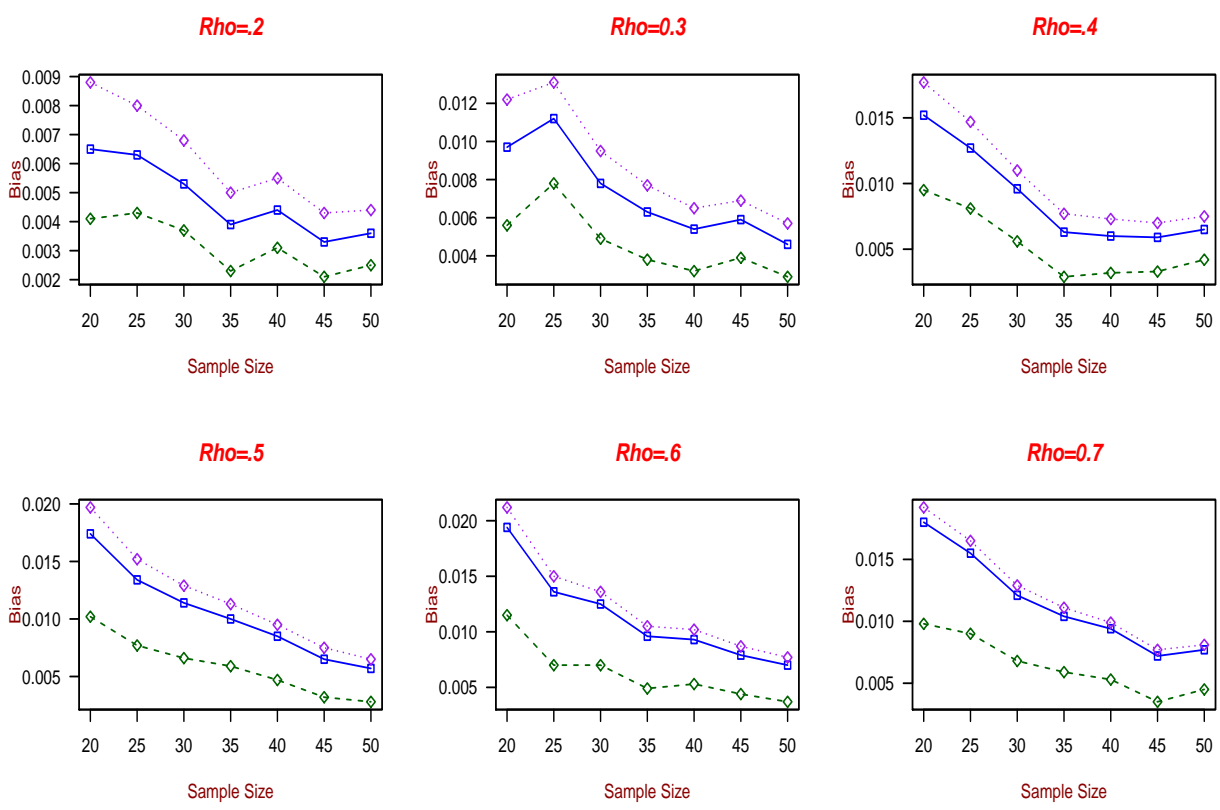

Rho $=.8$
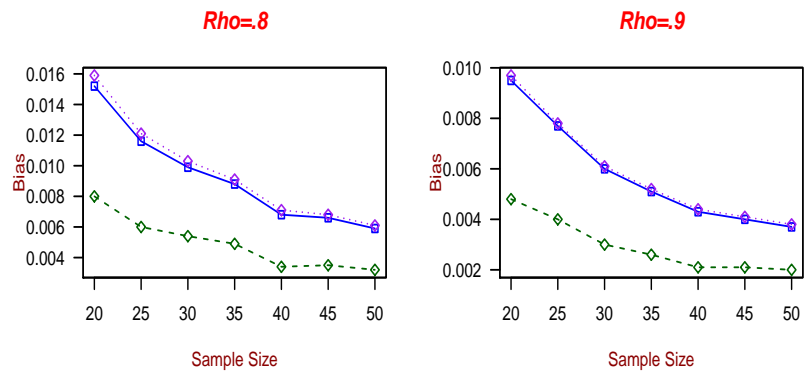

$\square \quad$ MLE

$-\diamond$ - Av.Pearson

@... Av.MLE

Figure 5.2: Comparison Bias Trivariate Normal Distribution 


\begin{tabular}{|c|c|c|c|c|c|c|c|c|}
\hline & \multirow{2}{*}{ Rho } & \multicolumn{7}{|c|}{ SAMPLE SIZE } \\
\hline & & 20 & 25 & 30 & 35 & 40 & 45 & 50 \\
\hline \multirow{14}{*}{$\tilde{\sigma}$} & -0.4 & 0.0015 & 0.0012 & 0.0010 & 0.0008 & 0.0007 & 0.0006 & 0.0006 \\
\hline & -0.3 & 0.0050 & 0.0039 & 0.0032 & 0.0027 & 0.0024 & 0.0021 & 0.0019 \\
\hline & -0.2 & 0.0093 & 0.0073 & 0.0060 & 0.0051 & 0.0045 & 0.0040 & 0.0035 \\
\hline & -0.1 & 0.0134 & 0.0107 & 0.0087 & 0.0073 & 0.0066 & 0.0058 & 0.0051 \\
\hline & 0 & 0.0168 & 0.0134 & 0.0114 & 0.0097 & 0.0083 & 0.0076 & 0.0067 \\
\hline & 0.1 & 0.0195 & 0.0155 & 0.0130 & 0.0110 & 0.0099 & 0.0088 & 0.0075 \\
\hline & 0.2 & 0.0211 & 0.0171 & 0.0146 & 0.0121 & 0.0107 & 0.0093 & 0.0085 \\
\hline & 0.3 & 0.0212 & 0.0175 & 0.0144 & 0.0123 & 0.0106 & 0.0095 & 0.0086 \\
\hline & 0.4 & 0.0204 & 0.0164 & 0.0134 & 0.0116 & 0.0101 & 0.0090 & 0.0080 \\
\hline & 0.5 & 0.0186 & 0.0140 & 0.0119 & 0.0103 & 0.0087 & 0.0078 & 0.0068 \\
\hline & 0.6 & 0.0153 & 0.0115 & 0.0095 & 0.0079 & 0.0069 & 0.0061 & 0.0056 \\
\hline & 0.7 & 0.0106 & 0.0084 & 0.0065 & 0.0055 & 0.0050 & 0.0042 & 0.0037 \\
\hline & 0.8 & 0.0060 & 0.0044 & 0.0037 & 0.0030 & 0.0026 & 0.0023 & 0.0020 \\
\hline & 0.9 & 0.0019 & 0.0014 & 0.0011 & 0.0009 & 0.0008 & 0.0007 & 0.0006 \\
\hline \multirow{14}{*}{$\overline{2}$} & -0.4 & 0.0017 & 0.0012 & 0.0010 & 0.0008 & 0.0007 & 0.0006 & 0.0006 \\
\hline & -0.3 & 0.0051 & 0.0040 & 0.0033 & 0.0027 & 0.0024 & 0.0021 & 0.0019 \\
\hline & -0.2 & 0.0093 & 0.0074 & 0.0061 & 0.0052 & 0.0045 & 0.0040 & 0.0036 \\
\hline & -0.1 & 0.0137 & 0.0109 & 0.0088 & 0.0074 & 0.0067 & 0.0058 & 0.0052 \\
\hline & 0 & 0.0173 & 0.0138 & 0.0116 & 0.0099 & 0.0085 & 0.0077 & 0.0068 \\
\hline & 0.1 & 0.0203 & 0.0161 & 0.0134 & 0.0113 & 0.0101 & 0.0089 & 0.0076 \\
\hline & 0.2 & 0.0220 & 0.0177 & 0.0150 & 0.0124 & 0.0110 & 0.0095 & 0.0087 \\
\hline & 0.3 & 0.0219 & 0.0181 & 0.0148 & 0.0126 & 0.0108 & 0.0097 & 0.0087 \\
\hline & 0.4 & 0.0211 & 0.0169 & 0.0137 & 0.0118 & 0.0103 & 0.0091 & 0.0081 \\
\hline & 0.5 & 0.0191 & 0.0143 & 0.0120 & 0.0104 & 0.0088 & 0.0078 & 0.0068 \\
\hline & 0.6 & 0.0154 & 0.0116 & 0.0095 & 0.0079 & 0.0069 & 0.0061 & 0.0056 \\
\hline & 0.7 & 0.0104 & 0.0082 & 0.0064 & 0.0054 & 0.0049 & 0.0041 & 0.0037 \\
\hline & 0.8 & 0.0057 & 0.0042 & 0.0036 & 0.0029 & 0.0026 & 0.0023 & 0.0020 \\
\hline & 0.9 & 0.0018 & 0.0014 & 0.0010 & 0.0009 & 0.0008 & 0.0007 & 0.0006 \\
\hline \multirow{14}{*}{$\tilde{u}$} & -0.4 & 0.0020 & 0.0014 & 0.0012 & 0.0009 & 0.0008 & 0.0007 & 0.0006 \\
\hline & -0.3 & 0.0051 & 0.0040 & 0.0033 & 0.0027 & 0.0024 & 0.0022 & 0.0019 \\
\hline & -0.2 & 0.0090 & 0.0071 & 0.0059 & 0.0051 & 0.0044 & 0.0040 & 0.0035 \\
\hline & -0.1 & 0.0131 & 0.0105 & 0.0086 & 0.0072 & 0.0066 & 0.0057 & 0.0051 \\
\hline & 0 & 0.0165 & 0.0132 & 0.0113 & 0.0096 & 0.0083 & 0.0075 & 0.0066 \\
\hline & 0.1 & 0.0193 & 0.0155 & 0.0130 & 0.0110 & 0.0099 & 0.0087 & 0.0075 \\
\hline & 0.2 & 0.0210 & 0.0171 & 0.0146 & 0.0121 & 0.0107 & 0.0093 & 0.0085 \\
\hline & 0.3 & 0.0212 & 0.0176 & 0.0144 & 0.0124 & 0.0106 & 0.0096 & 0.0086 \\
\hline & 0.4 & 0.0207 & 0.0166 & 0.0135 & 0.0117 & 0.0102 & 0.0090 & 0.0081 \\
\hline & 0.5 & 0.0190 & 0.0142 & 0.0120 & 0.0104 & 0.0087 & 0.0078 & 0.0068 \\
\hline & 0.6 & 0.0156 & 0.0117 & 0.0096 & 0.0080 & 0.0070 & 0.0061 & 0.0057 \\
\hline & 0.7 & 0.0108 & 0.0085 & 0.0066 & 0.0056 & 0.0050 & 0.0042 & 0.0037 \\
\hline & 0.8 & 0.0061 & 0.0044 & 0.0038 & 0.0031 & 0.0026 & 0.0024 & 0.0020 \\
\hline & 0.9 & 0.0020 & 0.0015 & 0.0011 & 0.0009 & 0.0008 & 0.0007 & 0.0006 \\
\hline
\end{tabular}

Table 5.3: Comparison MSE of Estimators Trivariate 
Rho= -.4

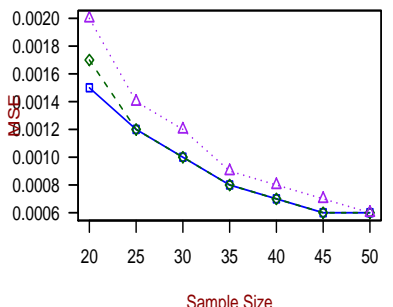

Rho=- 1

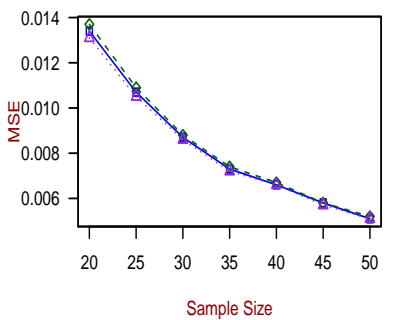

Rho $=.2$

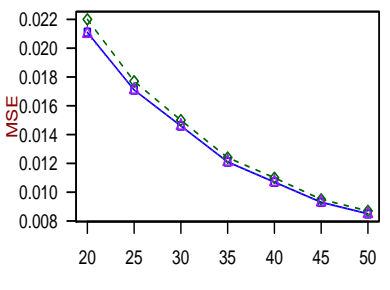

Sample Size

Rho $=.5$

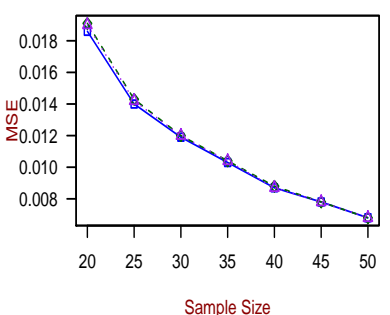

Rho $=.8$

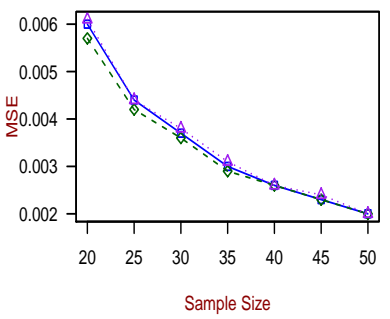

Rho $=-.3$

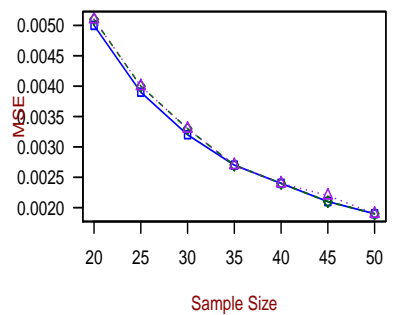

Rho $=0$

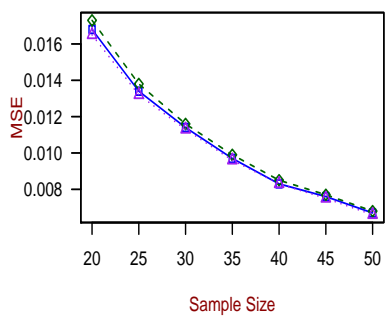

Rho $=0.3$

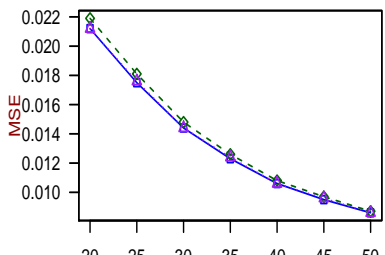

Sample Size

Rho $=6$

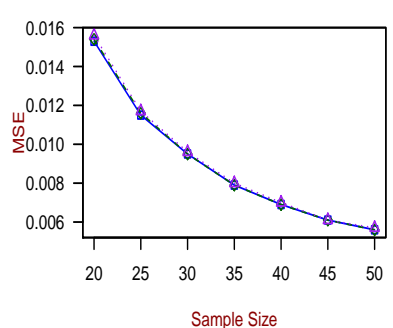

Rho $=.9$

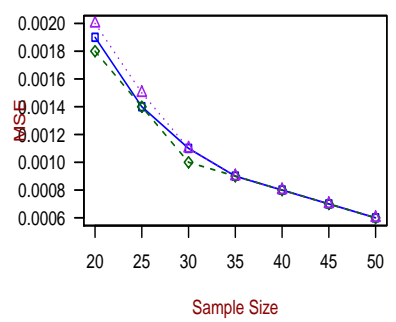

Rho $=-.2$

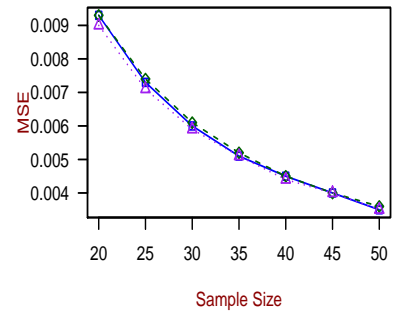

Rho $=.1$

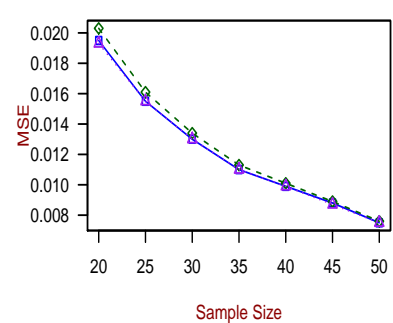

Rho=.4

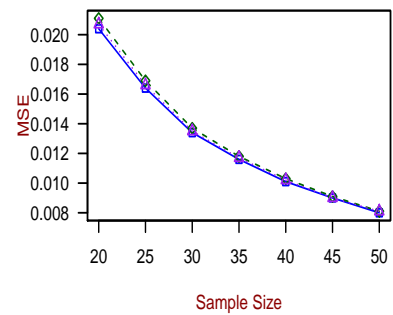

$R h o=0.7$

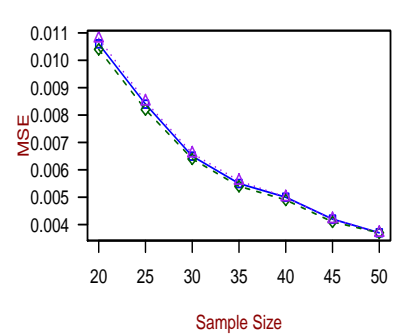

$\square \quad$ MLE

- $\diamond$ - Av.Pearson

$\triangle$ Av.MLE

Figure 5.3: Comparison MSE Trivariate Normal Distribution 


\subsection{Confidence Intervals}

In Section 3.3 was mentioned that

$$
\hat{\rho} \sim N\left(\rho, \frac{\left(1-\rho^{2}\right)^{2}(1+2 \rho)^{2}}{3 n}\right),
$$

therefore a $(1-\alpha) 100 \%$ confidence interval for $\rho$ is given by

$$
\hat{\rho} \pm z_{\alpha / 2} \frac{(1-\hat{\rho})(1+2 \hat{\rho})}{\sqrt{3 n}}
$$

Moreover, using the variance stabilizing transformation in Section 3.3.2 was found that

$$
\frac{1}{\sqrt{3}} \ln \left(\frac{1+2 \hat{\rho}}{1-\hat{\rho}}\right) \sim N\left(\frac{1}{\sqrt{3}} \ln \left(\frac{1+2 \rho}{1-\rho}\right), \frac{1}{n}\right)
$$

thus, a $(1-\alpha) 100 \%$ confidence interval for $\frac{1}{\sqrt{3}} \ln \left(\frac{1+2 \rho}{1-\rho}\right)$ is expressed by

$$
\frac{1}{\sqrt{3}} \ln \left(\frac{1+2 \hat{\rho}}{1-\hat{\rho}}\right) \pm z_{\alpha / 2} \frac{1}{\sqrt{n}}
$$

Since our purpose is to find a confidence interval for $\rho$, it can be made by applying inverse transformation and solve for $\rho$, finding the $(1-\alpha) 100 \%$ given by

$$
\left(\frac{e^{\sqrt{3} l}-1}{2+e^{\sqrt{3} l}}, \frac{e^{\sqrt{3} u}-1}{2+e^{\sqrt{3} u}}\right)
$$

where

$$
l=\frac{1}{\sqrt{3}} \ln \left(\frac{1+2 \hat{\rho}}{1-\hat{\rho}}\right)-z_{\alpha / 2} \frac{1}{\sqrt{n}}
$$

and

$$
u=\frac{1}{\sqrt{3}} \ln \left(\frac{1+2 \hat{\rho}}{1-\hat{\rho}}\right)+z_{\alpha / 2} \frac{1}{\sqrt{n}}
$$

However, using the expressions in (5.1) and (5.2) the $(1-\alpha) 100$ level of confidence is not achieved. For this reason, a study in the denominators of the sampling errors will be performed. 


\subsubsection{Study on the denominators}

A 95\% confidence interval for $\rho$ is desired. For this purpose, the coverage probability of the intervals

$$
\hat{\rho} \pm 1.96 \frac{(1-\hat{\rho})(1+2 \hat{\rho})}{\sqrt{3(n-k)}}
$$

for $k=0,1,2,3,4,5$ is calculated and recorded in Tables 5.4 using the MLE. 


\begin{tabular}{|c|c|c|c|c|c|c|c|}
\hline \multirow[b]{2}{*}{ RHO } & \multirow[b]{2}{*}{\begin{tabular}{|c|} 
SAMPLE \\
SIZE \\
\end{tabular}} & \multicolumn{6}{|c|}{ DENOMINADOR } \\
\hline & & $\mathbf{n}$ & $n-1$ & $n-2$ & $n-3$ & $n-4$ & $n-5$ \\
\hline \multirow{7}{*}{-0.4} & 20 & 0.9093 & 0.9153 & 0.9202 & 0.9256 & 0.9297 & 0.9339 \\
\hline & 25 & 0.9204 & 0.9253 & 0.9289 & 0.9321 & 0.9359 & 0.9396 \\
\hline & 30 & 0.9249 & 0.9284 & 0.9313 & 0.9343 & 0.9374 & 0.9403 \\
\hline & 35 & 0.9262 & 0.9287 & 0.9320 & 0.9348 & 0.9377 & 0.9401 \\
\hline & 40 & 0.9301 & 0.9324 & 0.9346 & 0.9368 & 0.9397 & 0.9423 \\
\hline & 45 & 0.9329 & 0.9355 & 0.9378 & 0.9392 & 0.9408 & 0.9430 \\
\hline & 50 & 0.9359 & 0.9385 & 0.9406 & 0.9428 & 0.9453 & 0.9469 \\
\hline \multirow{7}{*}{-0.3} & 20 & 0.9110 & 0.9156 & 0.9205 & 0.9265 & 0.9331 & 0.9390 \\
\hline & 25 & 0.9162 & 0.9201 & 0.9243 & 0.9277 & 0.9309 & 0.9355 \\
\hline & 30 & 0.9279 & 0.9318 & 0.9353 & 0.9388 & 0.9412 & 0.9444 \\
\hline & 35 & 0.9311 & 0.9344 & 0.9380 & 0.9407 & 0.9438 & 0.9467 \\
\hline & 40 & 0.9328 & 0.9354 & 0.9385 & 0.9408 & 0.9430 & 0.9450 \\
\hline & 45 & 0.9302 & 0.9330 & 0.9361 & 0.9384 & 0.9411 & 0.9434 \\
\hline & 50 & 0.9350 & 0.9366 & 0.9391 & 0.9413 & 0.9433 & 0.9456 \\
\hline \multirow{7}{*}{-0.2} & 20 & 0.9051 & 0.9120 & 0.9167 & 0.9219 & 0.9283 & 0.9339 \\
\hline & 25 & 0.9194 & 0.9239 & 0.9281 & 0.9328 & 0.9366 & 0.9412 \\
\hline & 30 & 0.9247 & 0.9292 & 0.9334 & 0.9374 & 0.9413 & 0.9460 \\
\hline & 35 & 0.9250 & 0.9289 & 0.9315 & 0.9346 & 0.9367 & 0.9389 \\
\hline & 40 & 0.9324 & 0.9346 & 0.9378 & 0.9408 & 0.9430 & 0.9450 \\
\hline & 45 & 0.9272 & 0.9296 & 0.9317 & 0.9349 & 0.9371 & 0.9393 \\
\hline & 50 & 0.9348 & 0.9372 & 0.9394 & 0.9417 & 0.9438 & 0.9458 \\
\hline \multirow{7}{*}{-0.1} & 20 & 9106 & 0.9159 & 0.9232 & 0.9295 & 0.9354 & 0.9420 \\
\hline & 25 & 0.9180 & 0.9221 & 0.9268 & 0.9317 & 0.9373 & 0.9421 \\
\hline & 30 & 0.9278 & 0.9315 & 0.9357 & 0.9394 & 0.9437 & 0.9467 \\
\hline & 35 & 0.9295 & 0.9322 & 0.9348 & 0.9386 & 0.9419 & 0.9458 \\
\hline & 40 & 0.9304 & 0.9331 & 0.9353 & 0.9379 & 0.9408 & 0.9435 \\
\hline & 45 & 0.9350 & 0.9374 & 0.9396 & 0.9420 & 0.9445 & 0.9467 \\
\hline & 50 & 0.9376 & 0.9401 & 0.9423 & 0.9442 & 0.9464 & 0.9490 \\
\hline \multirow{7}{*}{0} & 20 & .9149 & 0.9199 & 0.9263 & 0.9316 & 0.9365 & 0.9422 \\
\hline & 25 & 0.9202 & 0.9254 & 0.9301 & 0.9345 & 0.9402 & 0.9451 \\
\hline & 30 & 0.9201 & 0.9248 & 0.9285 & 0.9326 & 0.9368 & 0.9414 \\
\hline & 35 & 0.9271 & 0.9323 & 0.9365 & 0.9395 & 0.9439 & 0.9472 \\
\hline & 40 & 0.9340 & 0.9380 & 0.9413 & 0.9445 & 0.9470 & 0.9501 \\
\hline & 45 & 0.9296 & 0.9321 & 0.9348 & 0.9374 & 0.9396 & 0.9415 \\
\hline & 50 & 0.9380 & 0.9398 & 0.9425 & 0.9450 & 0.9469 & 0.9484 \\
\hline \multirow{7}{*}{0.1} & 20 & 0.9133 & 0.9194 & 0.9257 & 0.9323 & 0.9402 & 0.9468 \\
\hline & 25 & 0.9228 & 0.9276 & 0.9325 & 0.9378 & 0.9425 & 0.9470 \\
\hline & 30 & 0.9271 & 0.9313 & 0.9353 & 0.9393 & 0.9432 & 0.9483 \\
\hline & 35 & 0.9326 & 0.9359 & 0.9397 & 0.9430 & 0.9462 & 0.9490 \\
\hline & 40 & 0.9321 & 0.9348 & 0.9371 & 0.9405 & 0.9432 & 0.9457 \\
\hline & 45 & 0.9318 & 0.9341 & 0.9368 & 0.9404 & 0.9430 & 0.9456 \\
\hline & 50 & 0.9408 & 0.9436 & 0.9453 & 0.9470 & 0.9501 & 0.9522 \\
\hline \multirow{7}{*}{0.2} & 20 & 0.9184 & 0.9240 & 0.9290 & 0.9359 & 0.9399 & 0.9453 \\
\hline & 25 & 0.9198 & 0.9256 & 0.9305 & 0.9354 & 0.9404 & 0.9450 \\
\hline & 30 & 0.9205 & 0.9247 & 0.9288 & 0.9338 & 0.9381 & 0.9411 \\
\hline & 35 & 0.9282 & 0.9313 & 0.9355 & 0.9386 & 0.9423 & 0.9459 \\
\hline & 40 & 0.9311 & 0.9343 & 0.9363 & 0.9386 & 0.9423 & 0.9449 \\
\hline & 45 & 0.9372 & 0.9395 & 0.9428 & 0.9459 & 0.9489 & 0.9512 \\
\hline & 50 & 0.9331 & 0.9345 & 0.9369 & 0.9398 & 0.9422 & 0.9458 \\
\hline
\end{tabular}

\begin{tabular}{|c|c|c|c|c|c|c|c|}
\hline \multirow[b]{2}{*}{ RHO } & \multirow[b]{2}{*}{$\begin{array}{c}\text { SAMPLE } \\
\text { SIZE } \\
\end{array}$} & \multicolumn{6}{|c|}{ DENOMINADOR } \\
\hline & & $\mathbf{n}$ & $n-1$ & $\mathrm{n}-2$ & $n-3$ & $n-4$ & $n-5$ \\
\hline \multirow{7}{*}{0.3} & 20 & 0.9186 & 0.9244 & 0.9303 & 0.9369 & 0.9435 & 0.9502 \\
\hline & 25 & 0.9190 & 0.9238 & 0.9289 & 0.9336 & 0.9388 & 0.9433 \\
\hline & 30 & 0.9209 & 0.9260 & 0.9315 & 0.9368 & 0.9413 & 0.9468 \\
\hline & 35 & 0.9297 & 0.9335 & 0.9367 & 0.9401 & 0.9451 & 0.9484 \\
\hline & 40 & 0.9331 & 0.9366 & 0.9405 & 0.9435 & 0.9465 & 0.9504 \\
\hline & 45 & 0.9338 & 0.9360 & 0.9394 & 0.9418 & 0.9449 & 0.9472 \\
\hline & 50 & 0.9342 & 0.9369 & 0.9392 & 0.9421 & 0.9437 & 0.9460 \\
\hline \multirow{7}{*}{0.4} & 20 & 0.9196 & 0.9265 & 0.9332 & 0.9396 & 0.9474 & 0.9529 \\
\hline & 25 & 0.9241 & 0.9294 & 0.9330 & 0.9382 & 0.9430 & 0.9488 \\
\hline & 30 & 0.9304 & 0.9334 & 0.9370 & 0.9399 & 0.9432 & 0.9479 \\
\hline & 35 & 0.9319 & 0.9349 & 0.9380 & 0.9418 & 0.9457 & 0.9497 \\
\hline & 40 & 0.9324 & 0.9361 & 0.9389 & 0.9413 & 0.9429 & 0.9461 \\
\hline & 45 & 0.9350 & 0.9378 & 0.9408 & 0.9433 & 0.9456 & 0.9479 \\
\hline & 50 & 0.9378 & 0.9399 & 0.9424 & 0.9444 & 0.9473 & 0.9489 \\
\hline \multirow{7}{*}{0.5} & 20 & 0.9156 & 0.9218 & 0.9289 & 0.9346 & 0.9421 & 0.9493 \\
\hline & 25 & 0.9296 & 0.9337 & 0.9376 & 0.9423 & 0.9486 & 0.9538 \\
\hline & 30 & 0.9282 & 0.9328 & 0.9378 & 0.9426 & 0.9475 & 0.9515 \\
\hline & 35 & 0.9325 & 0.9368 & 0.9397 & 0.9434 & 0.9462 & 0.9495 \\
\hline & 40 & 0.9356 & 0.9391 & 0.9425 & 0.9451 & 0.9487 & 0.9516 \\
\hline & 45 & 0.9396 & 0.9417 & 0.9435 & 0.9465 & 0.9485 & 0.9511 \\
\hline & 50 & 0.9436 & 0.9464 & 0.9489 & 0.9506 & 0.9530 & 0.9543 \\
\hline \multirow{7}{*}{0.6} & 20 & 0.9178 & 0.9240 & 0.9311 & 0.9388 & 0.9444 & 0.9504 \\
\hline & 25 & 0.9293 & 0.9345 & 0.9402 & 0.9442 & 0.9490 & 0.9536 \\
\hline & 30 & 0.9295 & 0.9329 & 0.9379 & 0.9408 & 0.9453 & 0.9486 \\
\hline & 35 & 0.9353 & 0.9388 & 0.9415 & 0.9442 & 0.9475 & 0.9507 \\
\hline & 40 & 0.9401 & 0.9428 & 0.9450 & 0.9483 & 0.9497 & 0.9528 \\
\hline & 45 & 0.9403 & 0.9429 & 0.9457 & 0.9481 & 0.9510 & 0.9531 \\
\hline & 50 & 0.9359 & 0.9383 & 0.9415 & 0.9442 & 0.9466 & 0.9486 \\
\hline \multirow{7}{*}{0.7} & 20 & 0.9231 & 0.9295 & 0.9349 & 0.9410 & 0.9465 & 0.9531 \\
\hline & 25 & 0.9322 & 0.9371 & 0.9414 & 0.9475 & 0.9528 & 0.9571 \\
\hline & 30 & 0.9374 & 0.9427 & 0.9466 & 0.9489 & 0.9525 & 0.9559 \\
\hline & 35 & 0.9371 & 0.9402 & 0.9436 & 0.9478 & 0.9507 & 0.9535 \\
\hline & 40 & 0.9320 & 0.9347 & 0.9376 & 0.9410 & 0.9447 & 0.9475 \\
\hline & 45 & 0.9396 & 0.9425 & 0.9456 & 0.9473 & 0.9494 & 0.9517 \\
\hline & 50 & 0.9456 & 0.9479 & 0.9505 & 0.9529 & 0.9550 & 0.9566 \\
\hline \multirow{7}{*}{0.8} & 20 & 0.9272 & 0.9322 & 0.9372 & 0.9419 & 0.9474 & 0.9540 \\
\hline & 25 & 0.9356 & 0.9402 & 0.9451 & 0.9500 & 0.9535 & 0.9573 \\
\hline & 30 & 0.9334 & 0.9369 & 0.9405 & 0.9446 & 0.9483 & 0.9511 \\
\hline & 35 & 0.9369 & 0.9402 & 0.9438 & 0.9471 & 0.9501 & 0.9528 \\
\hline & 40 & 0.9382 & 0.9407 & 0.9423 & 0.9450 & 0.9481 & 0.9509 \\
\hline & 45 & 0.9393 & 0.9415 & 0.9440 & 0.9460 & 0.9481 & 0.9507 \\
\hline & 50 & 0.9398 & 0.9427 & 0.9452 & 0.9473 & 0.9488 & 0.9509 \\
\hline \multirow{7}{*}{0.9} & 20 & 0.9317 & 0.9368 & 0.9414 & 0.9466 & 0.9507 & 0.9556 \\
\hline & 25 & 0.9344 & 0.9394 & 0.9427 & 0.9467 & 0.9504 & 0.9551 \\
\hline & 30 & 0.9439 & 0.9462 & 0.9486 & 0.9503 & 0.9540 & 0.9567 \\
\hline & 35 & 0.9376 & 0.9403 & 0.9425 & 0.9460 & 0.9484 & 0.9506 \\
\hline & 40 & 0.9373 & 0.9393 & 0.9416 & 0.9443 & 0.9468 & 0.9496 \\
\hline & 45 & 0.9371 & 0.9403 & 0.9423 & 0.9446 & 0.9479 & 0.9505 \\
\hline & 50 & 0.9388 & 0.9409 & 0.9431 & 0.9443 & 0.9466 & 0.9480 \\
\hline
\end{tabular}

Table 5.4: Comparison Denominators MLE Using Normal Trivariate 

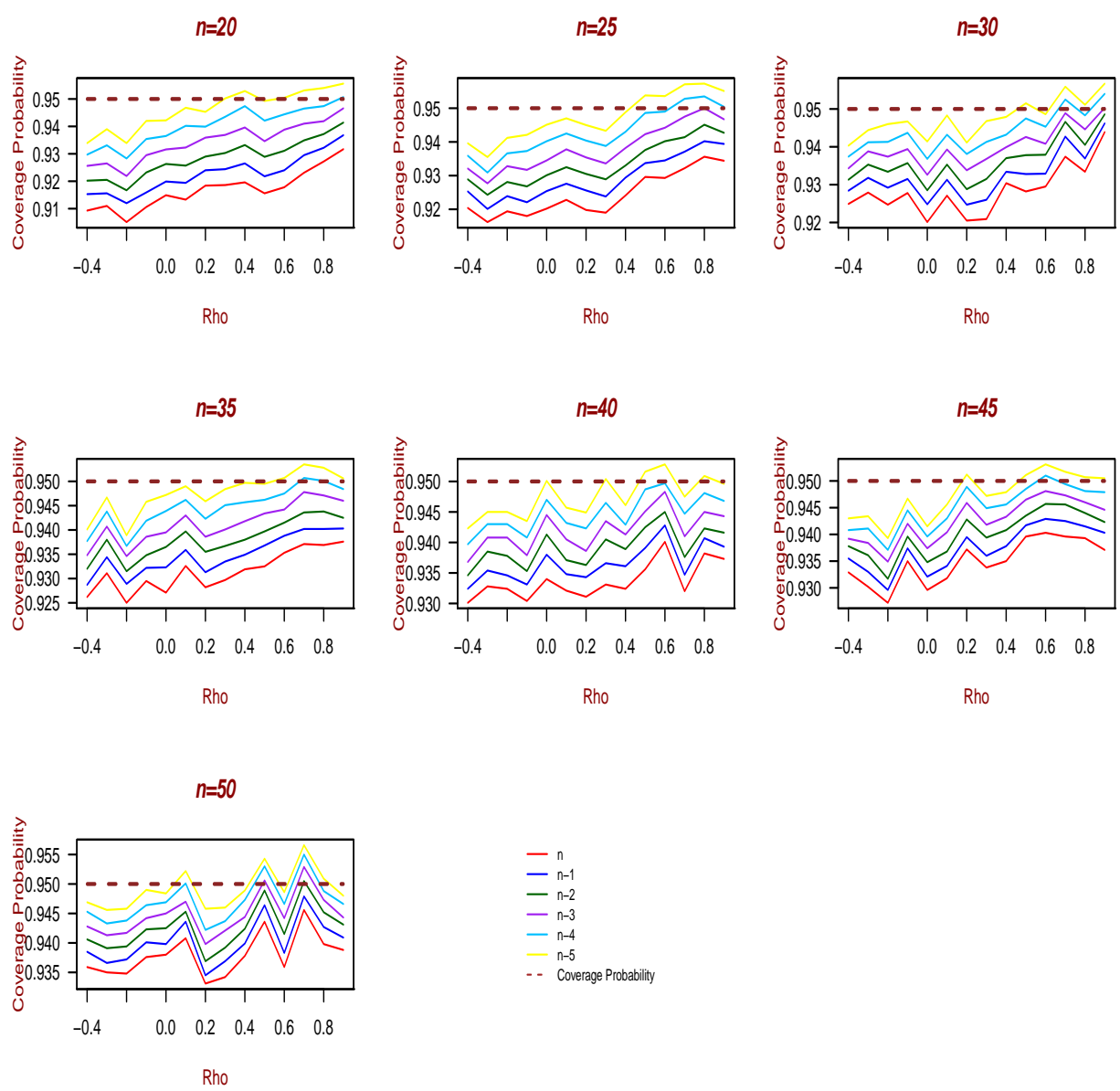

Figure 5.4: Comparison denominators using MLE in Expression 5.3

Figure 5.4 shows us that there is not an appropiate denominator to achieved the desired level of confidence when using the MLE as estimator of $\rho$.

For this reason, the confidence interval given in 5.1 will not be used further.

Now, using the expression in (5.2), a similar study on the denominators is performed; that is, $l$ and $u$ in this expression will be modified as

$$
l=\frac{1}{\sqrt{3}} \ln \left(\frac{1+2 \hat{\rho}}{1-\hat{\rho}}\right)-z_{\alpha / 2} \frac{1}{\sqrt{n-k}}
$$

and

$$
u=\frac{1}{\sqrt{3}} \ln \left(\frac{1+2 \hat{\rho}}{1-\hat{\rho}}\right)+z_{\alpha / 2} \frac{1}{\sqrt{n-k}}
$$


for $k$ ranging from 0 to five, and finally, an inverse transformation is apply to find the $95 \%$ confidence inteval for $\rho$ when $\hat{\rho}$ is the MLE. 


\begin{tabular}{|c|c|c|c|c|c|c|c|}
\hline \multirow[b]{2}{*}{ RHO } & \multirow[b]{2}{*}{$\begin{array}{c}\text { SAMPLE } \\
\text { SIZE }\end{array}$} & \multicolumn{6}{|c|}{ DENOMINADOR } \\
\hline & & $\mathrm{n}$ & $n-1$ & $n-2$ & $n-3$ & $n-4$ & $n-5$ \\
\hline \multirow{7}{*}{-0.4} & 20 & 0.9385 & 0.9451 & 0.9506 & 0.9562 & 0.9616 & 0.9684 \\
\hline & 25 & 0.9452 & 0.9490 & 0.9536 & 0.9591 & 0.9631 & 0.9675 \\
\hline & 30 & 0.9437 & 0.9476 & 0.9515 & 0.9562 & 0.9600 & 0.9639 \\
\hline & 35 & 0.9470 & 0.9508 & 0.9536 & 0.9572 & 0.9602 & 0.9632 \\
\hline & 40 & 0.9484 & 0.9505 & 0.9535 & 0.9557 & 0.9587 & 0.9616 \\
\hline & 45 & 0.9446 & 0.9479 & 0.9501 & 0.9527 & 0.9571 & 0.9592 \\
\hline & 50 & 0.9461 & 0.9475 & 0.9500 & 0.9527 & 0.9550 & 0.9585 \\
\hline \multirow{7}{*}{-0.3} & 20 & 0.9396 & 0.9457 & 0.9516 & 0.9582 & 0.9641 & 0.9701 \\
\hline & 25 & 0.9382 & 0.9437 & 0.9484 & 0.9528 & 0.9584 & 0.9615 \\
\hline & 30 & 0.9425 & 0.9467 & 0.9512 & 0.9551 & 0.9594 & 0.9631 \\
\hline & 35 & 0.9484 & 0.9519 & 0.9554 & 0.9586 & 0.9613 & 0.9641 \\
\hline & 40 & 0.9452 & 0.9481 & 0.9516 & 0.9544 & 0.9578 & 0.9608 \\
\hline & 45 & 0.9450 & 0.9468 & 0.9490 & 0.9523 & 0.9544 & 0.9572 \\
\hline & 50 & 0.9445 & 0.9463 & 0.9486 & 0.9511 & 0.9527 & 0.9549 \\
\hline \multirow{7}{*}{-0.2} & 20 & 0.9315 & 0.9385 & 0.9453 & 0.9516 & 0.9577 & 0.9648 \\
\hline & 25 & 0.9378 & 0.9423 & 0.9485 & 0.9529 & 0.9587 & 0.9639 \\
\hline & 30 & 0.9445 & 0.9491 & 0.9531 & 0.9574 & 0.9612 & 0.9641 \\
\hline & 35 & 0.9408 & 0.9443 & 0.9476 & 0.9509 & 0.9538 & 0.9573 \\
\hline & 40 & 0.9432 & 0.9468 & 0.9496 & 0.9519 & 0.9558 & 0.9581 \\
\hline & 45 & 0.9387 & 0.9414 & 0.9443 & 0.9477 & 0.9498 & 0.9529 \\
\hline & 50 & 0.9448 & 0.9470 & 0.9487 & 0.9516 & 0.9533 & 0.9553 \\
\hline \multirow{7}{*}{-0.1} & 20 & 0.9378 & 0.9445 & 0.9503 & 0.9571 & 0.9616 & 0.9685 \\
\hline & 25 & 0.9416 & 0.9462 & 0.9503 & 0.9549 & 0.9593 & 0.9633 \\
\hline & 30 & 0.9433 & 0.9471 & 0.9513 & 0.9550 & 0.9580 & 0.9618 \\
\hline & 35 & 0.9470 & 0.9502 & 0.9531 & 0.9563 & 0.9597 & 0.9633 \\
\hline & 40 & 0.9420 & 0.9455 & 0.9490 & 0.9526 & 0.9554 & 0.9587 \\
\hline & 45 & 0.9440 & 0.9473 & 0.9493 & 0.9516 & 0.9552 & 0.9588 \\
\hline & 50 & 0.9506 & 0.9522 & 0.9544 & 0.9571 & 0.9593 & 0.9612 \\
\hline \multirow{7}{*}{0} & 20 & 0.9399 & 0.9451 & 0.9505 & 0.9569 & 0.9631 & 0.9671 \\
\hline & 25 & 0.9407 & 0.9462 & 0.9526 & 0.9584 & 0.9621 & 0.9658 \\
\hline & 30 & 0.9391 & 0.9434 & 0.9484 & 0.9527 & 0.9580 & 0.9622 \\
\hline & 35 & 0.9418 & 0.9450 & 0.9483 & 0.9525 & 0.9574 & 0.9601 \\
\hline & 40 & 0.9480 & 0.9511 & 0.9541 & 0.9571 & 0.9593 & 0.9620 \\
\hline & 45 & 0.9421 & 0.9437 & 0.9465 & 0.9483 & 0.9511 & 0.9536 \\
\hline & 50 & 0.9478 & 0.9494 & 0.9520 & 0.9544 & 0.9569 & 0.9592 \\
\hline \multirow{7}{*}{0.1} & 20 & 0.9384 & 0.9436 & 0.9493 & 0.9561 & 0.9611 & 0.9662 \\
\hline & 25 & 0.9421 & 0.9470 & 0.9526 & 0.9566 & 0.9607 & 0.9653 \\
\hline & 30 & 0.9435 & 0.9472 & 0.9505 & 0.9540 & 0.9578 & 0.9614 \\
\hline & 35 & 0.9462 & 0.9494 & 0.9534 & 0.9563 & 0.9589 & 0.9617 \\
\hline & 40 & 0.9440 & 0.9465 & 0.9497 & 0.9514 & 0.9542 & 0.9569 \\
\hline & 45 & 0.9436 & 0.9466 & 0.9491 & 0.9515 & 0.9544 & 0.9569 \\
\hline & 50 & 0.9493 & 0.9514 & 0.9543 & 0.9561 & 0.9590 & 0.9613 \\
\hline \multirow{7}{*}{0.2} & 20 & 0.9374 & 0.9422 & 0.9487 & 0.9554 & 0.9602 & 0.9654 \\
\hline & 25 & 0.9393 & 0.9442 & 0.9481 & 0.9536 & 0.9588 & 0.9626 \\
\hline & 30 & 0.9351 & 0.9388 & 0.9442 & 0.9491 & 0.9520 & 0.9561 \\
\hline & 35 & 0.9419 & 0.9454 & 0.9480 & 0.9508 & 0.9540 & 0.9573 \\
\hline & 40 & 0.9406 & 0.9439 & 0.9475 & 0.9502 & 0.9527 & 0.9560 \\
\hline & 45 & 0.9484 & 0.9508 & 0.9535 & 0.9550 & 0.9578 & 0.9607 \\
\hline & 50 & 0.9419 & 0.9444 & 0.9464 & 0.9503 & 0.9528 & 0.9551 \\
\hline
\end{tabular}

\begin{tabular}{|c|c|c|c|c|c|c|c|}
\hline \multirow{2}{*}{ RHO } & \multirow[b]{2}{*}{$\begin{array}{c}\text { SAMPLE } \\
\text { SIZE }\end{array}$} & \multicolumn{6}{|c|}{ DENOMINADOR } \\
\hline & & $\mathbf{n}$ & $n-1$ & $\mathrm{n}-2$ & $n-3$ & $n-4$ & $n-5$ \\
\hline \multirow{7}{*}{0.3} & 20 & 0.9384 & 0.9455 & 0.9513 & 0.9571 & 0.9628 & 0.9675 \\
\hline & 25 & 0.9357 & 0.9393 & 0.9441 & 0.9498 & 0.9560 & 0.9613 \\
\hline & 30 & 0.9387 & 0.9433 & 0.9477 & 0.9525 & 0.9574 & 0.9617 \\
\hline & 35 & 0.9425 & 0.9457 & 0.9501 & 0.9529 & 0.9558 & 0.9592 \\
\hline & 40 & 0.9447 & 0.9479 & 0.9516 & 0.9547 & 0.9585 & 0.9611 \\
\hline & 45 & 0.9436 & 0.9457 & 0.9483 & 0.9510 & 0.9537 & 0.9566 \\
\hline & 50 & 0.9431 & 0.9457 & 0.9472 & 0.9492 & 0.9512 & 0.9543 \\
\hline \multirow{7}{*}{0.4} & 20 & 0.9407 & 0.9465 & 0.9515 & 0.9585 & 0.9630 & 0.9694 \\
\hline & 25 & 0.9388 & 0.9443 & 0.9493 & 0.9539 & 0.9584 & 0.9625 \\
\hline & 30 & 0.9417 & 0.9454 & 0.9493 & 0.9516 & 0.9551 & 0.9595 \\
\hline & 35 & 0.9445 & 0.9485 & 0.9514 & 0.9545 & 0.9583 & 0.9616 \\
\hline & 40 & 0.9410 & 0.9442 & 0.9475 & 0.9506 & 0.9530 & 0.9556 \\
\hline & 45 & 0.9429 & 0.9454 & 0.9480 & 0.9510 & 0.9532 & 0.9555 \\
\hline & 50 & 0.9444 & 0.9466 & 0.9484 & 0.9509 & 0.9534 & 0.9561 \\
\hline \multirow{7}{*}{0.5} & 20 & 0.9351 & 0.9413 & 0.9471 & 0.9534 & 0.9597 & 0.9655 \\
\hline & 25 & 0.9427 & 0.9481 & 0.9533 & 0.9564 & 0.9613 & 0.9667 \\
\hline & 30 & 0.9410 & 0.9453 & 0.9496 & 0.9540 & 0.9576 & 0.9613 \\
\hline & 35 & 0.9426 & 0.9457 & 0.9488 & 0.9520 & 0.9549 & 0.9581 \\
\hline & 40 & 0.9455 & 0.9481 & 0.9510 & 0.9541 & 0.9562 & 0.9589 \\
\hline & 45 & 0.9470 & 0.9499 & 0.9526 & 0.9546 & 0.9584 & 0.9602 \\
\hline & 50 & 0.9469 & 0.9495 & 0.9522 & 0.9550 & 0.9572 & 0.9590 \\
\hline \multirow{7}{*}{0.6} & 20 & 0.9337 & 0.9400 & 0.9460 & 0.9510 & 0.9584 & 0.9649 \\
\hline & 25 & 0.9406 & 0.9462 & 0.9514 & 0.9572 & 0.9616 & 0.9665 \\
\hline & 30 & 0.9415 & 0.9455 & 0.9498 & 0.9534 & 0.9575 & 0.9617 \\
\hline & 35 & 0.9426 & 0.9465 & 0.9496 & 0.9530 & 0.9562 & 0.9591 \\
\hline & 40 & 0.9460 & 0.9476 & 0.9517 & 0.9541 & 0.9571 & 0.9603 \\
\hline & 45 & 0.9459 & 0.9486 & 0.9508 & 0.9539 & 0.9568 & 0.9597 \\
\hline & 50 & 0.9443 & 0.9467 & 0.9488 & 0.9517 & 0.9537 & 0.9552 \\
\hline \multirow{7}{*}{0.7} & 20 & 0.9382 & 0.9445 & 0.9516 & 0.9565 & 0.9624 & 0.9680 \\
\hline & 25 & 0.9416 & 0.9456 & 0.9500 & 0.9544 & 0.9590 & 0.9645 \\
\hline & 30 & 0.9436 & 0.9484 & 0.9523 & 0.9563 & 0.9592 & 0.9624 \\
\hline & 35 & 0.9419 & 0.9457 & 0.9490 & 0.9533 & 0.9565 & 0.9598 \\
\hline & 40 & 0.9378 & 0.9410 & 0.9447 & 0.9486 & 0.9518 & 0.9553 \\
\hline & 45 & 0.9459 & 0.9482 & 0.9506 & 0.9537 & 0.9568 & 0.9591 \\
\hline & 50 & 0.9497 & 0.9515 & 0.9538 & 0.9566 & 0.9589 & 0.9606 \\
\hline \multirow{7}{*}{0.8} & 20 & 0.9374 & 0.9443 & 0.9502 & 0.9555 & 0.9608 & 0.9665 \\
\hline & 25 & 0.9435 & 0.9480 & 0.9523 & 0.9563 & 0.9613 & 0.9653 \\
\hline & 30 & 0.9416 & 0.9454 & 0.9491 & 0.9530 & 0.9568 & 0.9600 \\
\hline & 35 & 0.9432 & 0.9463 & 0.9498 & 0.9532 & 0.9563 & 0.9599 \\
\hline & 40 & 0.9443 & 0.9472 & 0.9498 & 0.9527 & 0.9562 & 0.9592 \\
\hline & 45 & 0.9387 & 0.9421 & 0.9447 & 0.9475 & 0.9499 & 0.9536 \\
\hline & 50 & 0.9452 & 0.9473 & 0.9491 & 0.9513 & 0.9539 & 0.9554 \\
\hline \multirow{7}{*}{0.9} & 20 & 0.9375 & 0.9442 & 0.9504 & 0.9569 & 0.9622 & 0.9667 \\
\hline & 25 & 0.9408 & 0.9459 & 0.9516 & 0.9568 & 0.9612 & 0.9650 \\
\hline & 30 & 0.9447 & 0.9487 & 0.9523 & 0.9560 & 0.9594 & 0.9636 \\
\hline & 35 & 0.9391 & 0.9429 & 0.9463 & 0.9495 & 0.9536 & 0.9574 \\
\hline & 40 & 0.9408 & 0.9440 & 0.9469 & 0.9495 & 0.9526 & 0.9567 \\
\hline & 45 & 0.9419 & 0.9453 & 0.9478 & 0.9502 & 0.9527 & 0.9552 \\
\hline & 50 & 0.9397 & 0.9418 & 0.9444 & 0.9458 & 0.9485 & 0.9514 \\
\hline
\end{tabular}

Table 5.5: Comparison Denominators MLE Using Variance Stabilizing Transformation Trivariate 


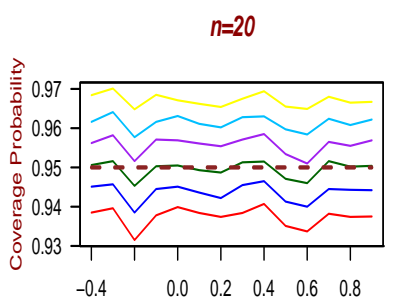

Rho

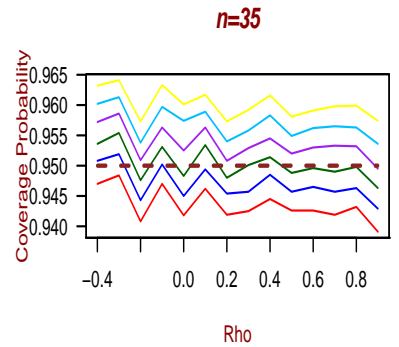

Rho

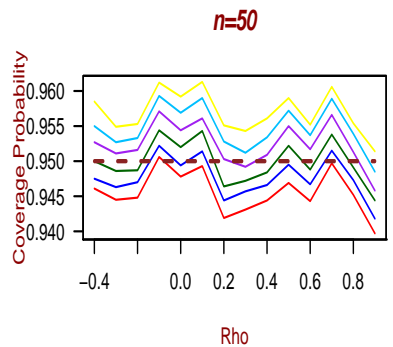

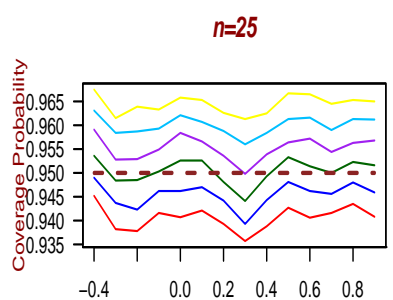

Rho

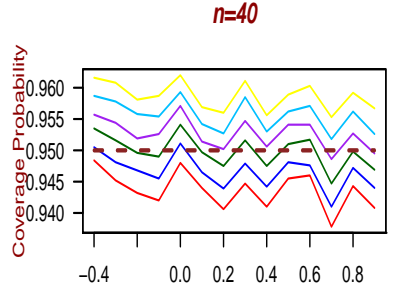

Rho

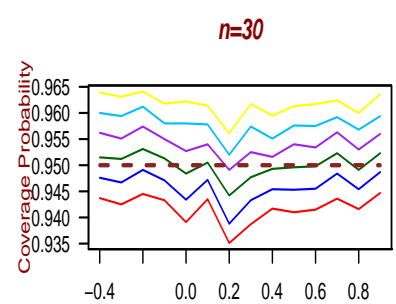

Rho

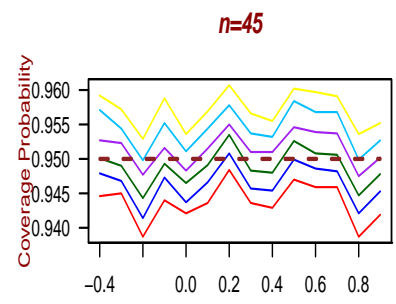

Rho $-n$

$-n-1$

- ${ }^{n-2}-3$

- ${ }^{n-3}-4$

$\begin{array}{ll} & n-5 \\ \text { - - } & \text { Coverage Probability }\end{array}$

Figure 5.5: Comparison denominator MLE using variance stabilizing transformation

The coverage probability of the confidence interval using the MLE is presented in Table 5.5 and Figure 5.5.

It can be notice is Figure 5.5 that $n-2$ is the best denominator to achieve the desired level of confidence. Therefore, it can be conclude that

$$
\frac{1}{\sqrt{3}} \ln \left(\frac{1+2 \hat{\rho}}{1-\hat{\rho}}\right) \sim N\left(\frac{1}{\sqrt{3}} \ln \left(\frac{1+2 \rho}{1-\rho}\right), \frac{1}{n-2}\right)
$$

is the more appropiate when using the MLE. 


\subsubsection{Confidence Intervals}

Once we have the more appropiate distributions of $\frac{1}{\sqrt{3}} \ln \left(\frac{1+2 \hat{\rho}_{A P}}{1-\hat{\rho}_{A P}}\right)$ when using the MLE, a confidence interval for $\rho$ can be found.

In this section the coverage probability of the confidence intervals is made. Moreover, the average width of those intervals are also measured. The results are presented in Tables 5.6 and 5.7, respectively.

\begin{tabular}{|c|c|c|c|c|c|c|c|}
\hline \multirow{2}{*}{ Rho } & \multicolumn{7}{|c|}{ SAMPLE SIZE } \\
\cline { 2 - 8 } & $\mathbf{2 0}$ & $\mathbf{2 5}$ & $\mathbf{3 0}$ & $\mathbf{3 5}$ & $\mathbf{4 0}$ & $\mathbf{4 5}$ & $\mathbf{5 0}$ \\
\hline $\mathbf{- 0 . 4}$ & 0.9506 & 0.9536 & 0.9515 & 0.9536 & 0.9535 & 0.9501 & 0.9500 \\
$\mathbf{- 0 . 3}$ & 0.9516 & 0.9484 & 0.9512 & 0.9554 & 0.9516 & 0.9490 & 0.9486 \\
$\mathbf{- 0 . 2}$ & 0.9453 & 0.9485 & 0.9531 & 0.9476 & 0.9496 & 0.9443 & 0.9487 \\
$\mathbf{- 0 . 1}$ & 0.9503 & 0.9503 & 0.9513 & 0.9531 & 0.9490 & 0.9493 & 0.9544 \\
$\mathbf{0}$ & 0.9505 & 0.9526 & 0.9484 & 0.9483 & 0.9541 & 0.9465 & 0.9520 \\
$\mathbf{0 . 1}$ & 0.9493 & 0.9526 & 0.9505 & 0.9534 & 0.9497 & 0.9491 & 0.9543 \\
$\mathbf{0 . 2}$ & 0.9487 & 0.9481 & 0.9442 & 0.9480 & 0.9475 & 0.9535 & 0.9464 \\
$\mathbf{0 . 3}$ & 0.9513 & 0.9441 & 0.9477 & 0.9501 & 0.9516 & 0.9483 & 0.9472 \\
$\mathbf{0 . 4}$ & 0.9515 & 0.9493 & 0.9493 & 0.9514 & 0.9475 & 0.9480 & 0.9484 \\
$\mathbf{0 . 5}$ & 0.9471 & 0.9533 & 0.9496 & 0.9488 & 0.9510 & 0.9526 & 0.9522 \\
$\mathbf{0 . 6}$ & 0.9460 & 0.9514 & 0.9498 & 0.9496 & 0.9517 & 0.9508 & 0.9488 \\
$\mathbf{0 . 7}$ & 0.9516 & 0.9500 & 0.9523 & 0.9490 & 0.9447 & 0.9506 & 0.9538 \\
$\mathbf{0 . 8}$ & 0.9502 & 0.9523 & 0.9491 & 0.9498 & 0.9498 & 0.9447 & 0.9491 \\
$\mathbf{0 . 9}$ & 0.9504 & 0.9516 & 0.9523 & 0.9463 & 0.9469 & 0.9478 & 0.9444 \\
\hline
\end{tabular}

Table 5.6: Coverage Probability Confidence Interval for $\rho$ Trivariate 


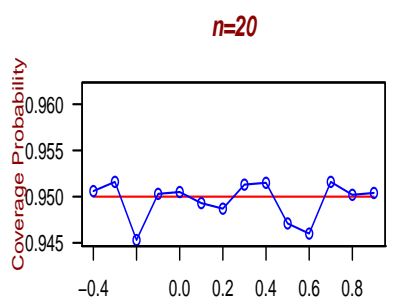

Rho

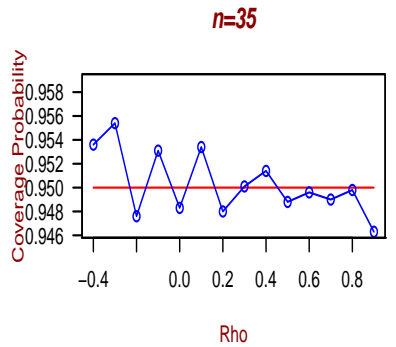

Rho

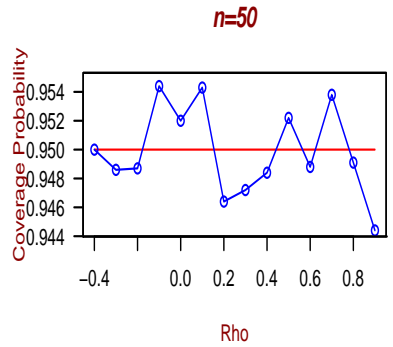

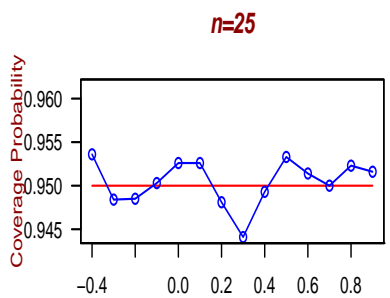

Rho

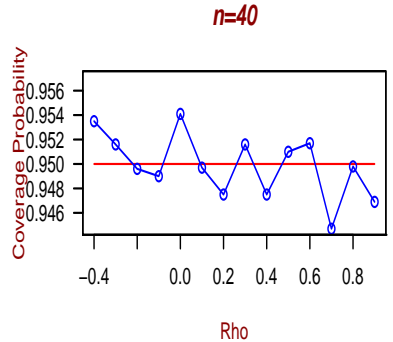

Rho

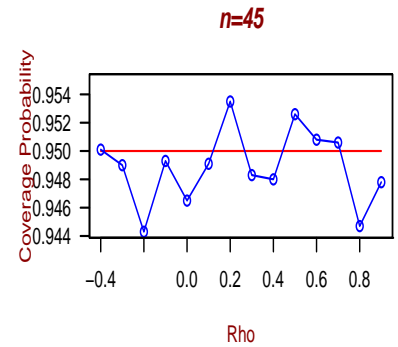

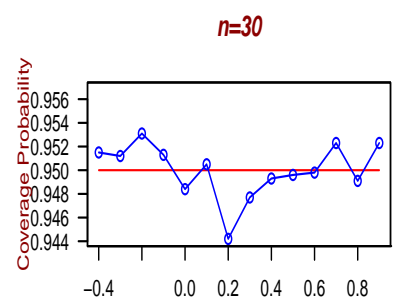

Rho

Figure 5.6: Comparison Coverage Probability of CI using Fisher

In Figure 5.6 it can be observed that the coverage probability of the confidence intervals are close to 0.95 . 


\begin{tabular}{|c|c|c|c|c|c|c|c|}
\hline \multirow{2}{*}{ Rho } & \multicolumn{7}{|c|}{ SAMPLE SIZE } \\
\cline { 2 - 8 } & $\mathbf{2 0}$ & $\mathbf{2 5}$ & $\mathbf{3 0}$ & $\mathbf{3 5}$ & $\mathbf{4 0}$ & $\mathbf{4 5}$ & $\mathbf{5 0}$ \\
\hline $\mathbf{- 0 . 4}$ & 0.1631 & 0.1413 & 0.1267 & 0.1155 & 0.1070 & 0.1004 & 0.0949 \\
$\mathbf{- 0 . 3}$ & 0.2853 & 0.2511 & 0.2279 & 0.2084 & 0.1945 & 0.1821 & 0.1722 \\
$\mathbf{- 0 . 2}$ & 0.3770 & 0.3361 & 0.3061 & 0.2820 & 0.2631 & 0.2463 & 0.2345 \\
$\mathbf{- 0 . 1}$ & 0.4492 & 0.4011 & 0.3648 & 0.3379 & 0.3162 & 0.2979 & 0.2826 \\
$\mathbf{0}$ & 0.4990 & 0.4477 & 0.4083 & 0.3794 & 0.3553 & 0.3350 & 0.3183 \\
$\mathbf{0 . 1}$ & 0.5322 & 0.4779 & 0.4384 & 0.4068 & 0.3810 & 0.3595 & 0.3421 \\
$\mathbf{0 . 2}$ & 0.5486 & 0.4934 & 0.4520 & 0.4201 & 0.3938 & 0.3722 & 0.3534 \\
$\mathbf{0 . 3}$ & 0.5500 & 0.4945 & 0.4532 & 0.4207 & 0.3945 & 0.3726 & 0.3539 \\
$\mathbf{0 . 4}$ & 0.5361 & 0.4812 & 0.4403 & 0.4079 & 0.3823 & 0.3609 & 0.3430 \\
$\mathbf{0 . 5}$ & 0.5052 & 0.4525 & 0.4131 & 0.3824 & 0.3579 & 0.3370 & 0.3198 \\
$\mathbf{0 . 6}$ & 0.4574 & 0.4066 & 0.3707 & 0.3419 & 0.3197 & 0.3008 & 0.2848 \\
$\mathbf{0 . 7}$ & 0.3885 & 0.3443 & 0.3113 & 0.2866 & 0.2668 & 0.2501 & 0.2373 \\
$\mathbf{0 . 8}$ & 0.2950 & 0.2580 & 0.2322 & 0.2131 & 0.1970 & 0.1849 & 0.1746 \\
$\mathbf{0 . 9}$ & 0.1697 & 0.1466 & 0.1303 & 0.1187 & 0.1095 & 0.1024 & 0.0965 \\
\hline
\end{tabular}

Table 5.7: Average Width of Confidence Interval for $\rho$ Trivariate 


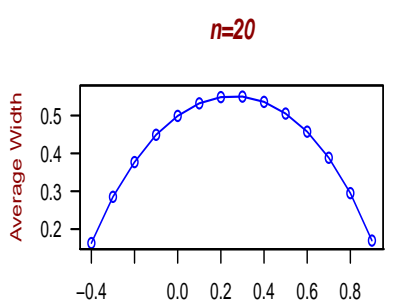

Rho

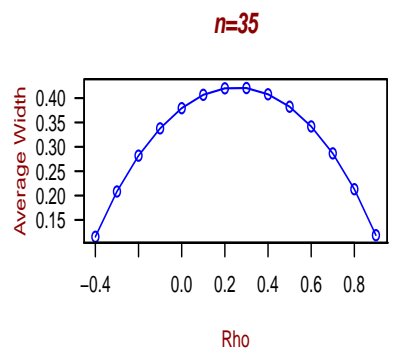

Rho

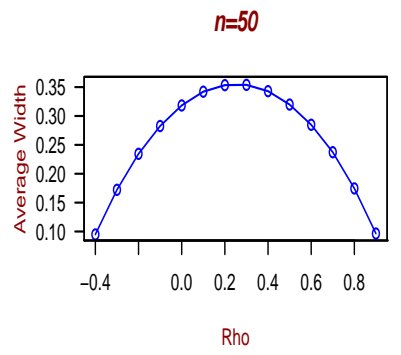

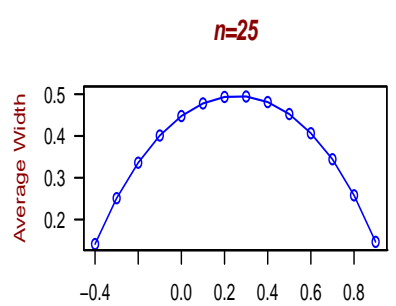

Rho

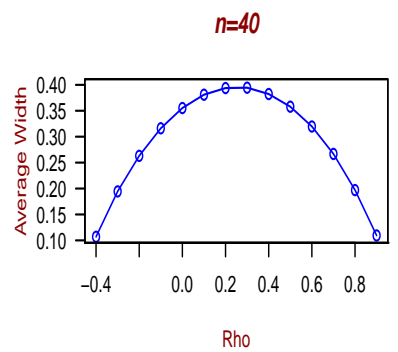

Rho

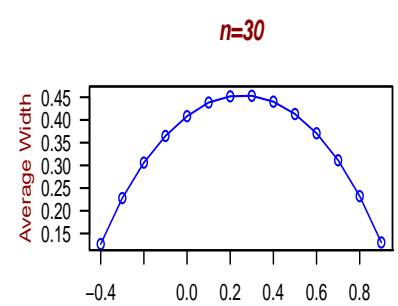

Rho

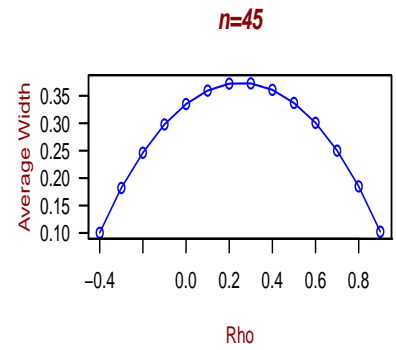

Figure 5.7: Comparison Width 95\% CI using Fisher

Figure 5.7 shows that the average width of the confidence intervals of $\rho$ is small when rho approaches to the extreme values.

\subsection{Test of Hypothesis}

\subsubsection{Probability Type I error}

A comparison of the probability of type I error for testing

$$
H_{0}: \rho=\rho_{0} \quad \text { vs. } \quad H a: \rho \neq \rho_{0}
$$


where $\rho_{0}$ is the true value of $\rho$ are made using the MLE in Table 5.8 using a significance level of $\alpha=0.05$

\begin{tabular}{|c|c|c|c|c|c|c|c|}
\hline \multirow{2}{*}{ Rho } & \multicolumn{7}{|c|}{ SAMPLE SIZE } \\
\cline { 2 - 8 } & $\mathbf{2 0}$ & $\mathbf{2 5}$ & $\mathbf{3 0}$ & $\mathbf{3 5}$ & $\mathbf{4 0}$ & $\mathbf{4 5}$ & $\mathbf{5 0}$ \\
\hline $\mathbf{- 0 . 4}$ & 0.0494 & 0.0464 & 0.0485 & 0.0464 & 0.0465 & 0.0499 & 0.0500 \\
$\mathbf{- 0 . 3}$ & 0.0484 & 0.0516 & 0.0488 & 0.0446 & 0.0484 & 0.0510 & 0.0514 \\
$\mathbf{- 0 . 2}$ & 0.0547 & 0.0515 & 0.0469 & 0.0524 & 0.0504 & 0.0557 & 0.0513 \\
$\mathbf{- 0 . 1}$ & 0.0497 & 0.0497 & 0.0487 & 0.0469 & 0.0510 & 0.0507 & 0.0456 \\
$\mathbf{0}$ & 0.0495 & 0.0474 & 0.0516 & 0.0517 & 0.0459 & 0.0535 & 0.0480 \\
$\mathbf{0 . 1}$ & 0.0507 & 0.0474 & 0.0495 & 0.0466 & 0.0503 & 0.0509 & 0.0457 \\
$\mathbf{0 . 2}$ & 0.0513 & 0.0519 & 0.0558 & 0.0520 & 0.0525 & 0.0465 & 0.0536 \\
$\mathbf{0 . 3}$ & 0.0487 & 0.0559 & 0.0523 & 0.0499 & 0.0484 & 0.0517 & 0.0528 \\
$\mathbf{0 . 4}$ & 0.0485 & 0.0507 & 0.0507 & 0.0486 & 0.0525 & 0.0520 & 0.0516 \\
$\mathbf{0 . 5}$ & 0.0529 & 0.0467 & 0.0504 & 0.0512 & 0.0490 & 0.0474 & 0.0478 \\
$\mathbf{0 . 6}$ & 0.0540 & 0.0486 & 0.0502 & 0.0504 & 0.0483 & 0.0492 & 0.0512 \\
$\mathbf{0 . 7}$ & 0.0484 & 0.0500 & 0.0477 & 0.0510 & 0.0553 & 0.0494 & 0.0462 \\
$\mathbf{0 . 8}$ & 0.0498 & 0.0477 & 0.0509 & 0.0502 & 0.0502 & 0.0553 & 0.0509 \\
$\mathbf{0 . 9}$ & 0.0496 & 0.0484 & 0.0477 & 0.0537 & 0.0531 & 0.0522 & 0.0556 \\
\hline
\end{tabular}

Table 5.8: Type I error Trivariate 

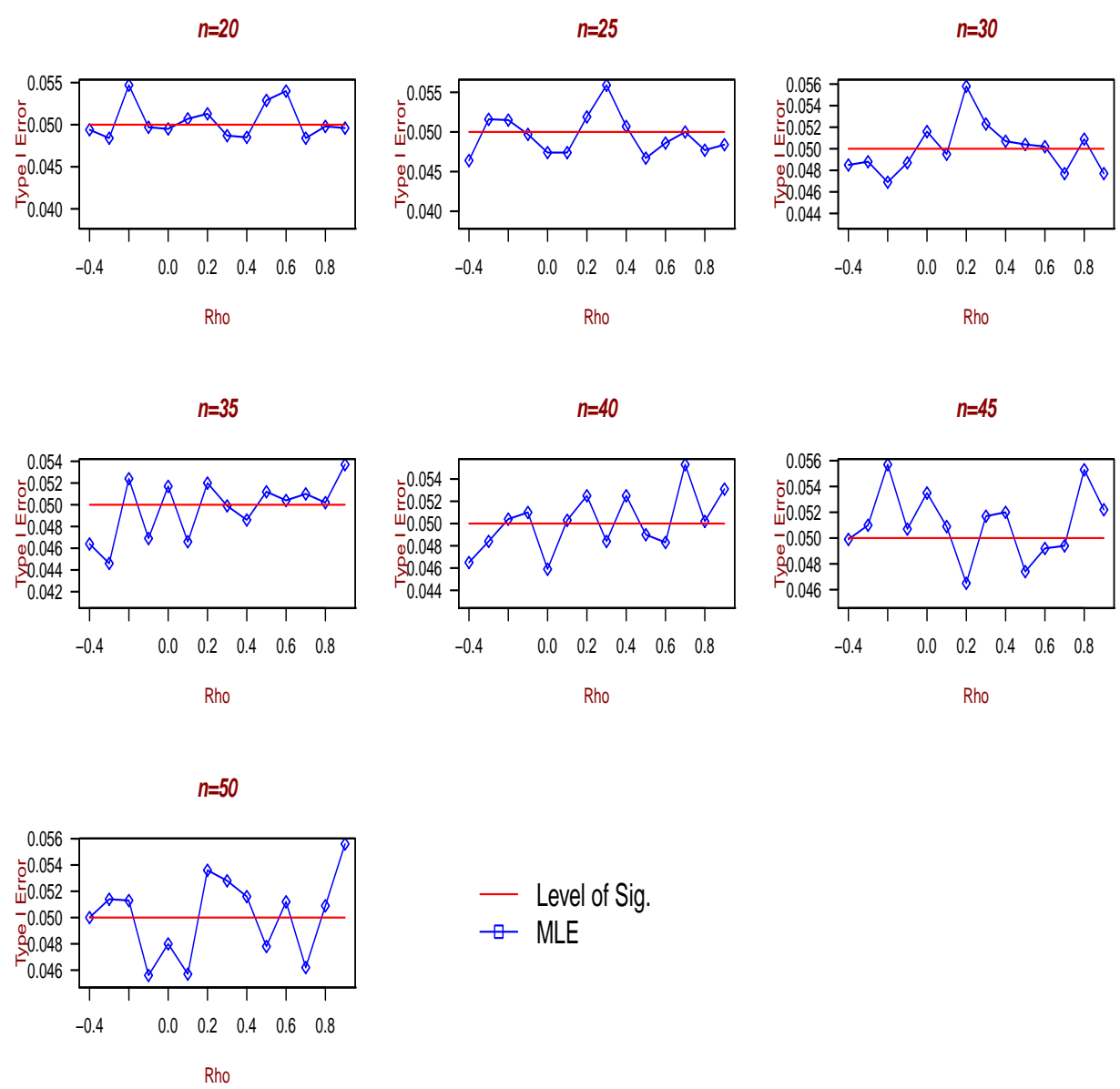

$$
\begin{aligned}
& - \text { Level of Sig. } \\
& \square \quad \text { MLE }
\end{aligned}
$$

Figure 5.8: Comparison Type I error

Figure 5.8 shows that the probability of the Type I error committed for the MLE is around 0.05, ranging between 0.044 and 0.056 .

\subsubsection{Power of the test}

In order to calculate the power when testing the hypotheses

$$
H_{0}: \rho=\rho_{0} \quad \text { vs. } \quad H_{a}: \rho=\rho_{1}
$$

when $\rho_{0}$ is the true value of $\rho$ and $\rho_{1} \neq \rho_{0}$ different simulations are conducted. Assuming that $\rho=\rho_{0}$ take the values from -0.3 to 0.9 in 0.1 increments, the values 
for $\rho_{1}$ in the alternative hypothesis were chosen as $\rho_{1}=\rho_{0} \pm 0.10$. Table 5.9 presents the values of the power using the MLE.

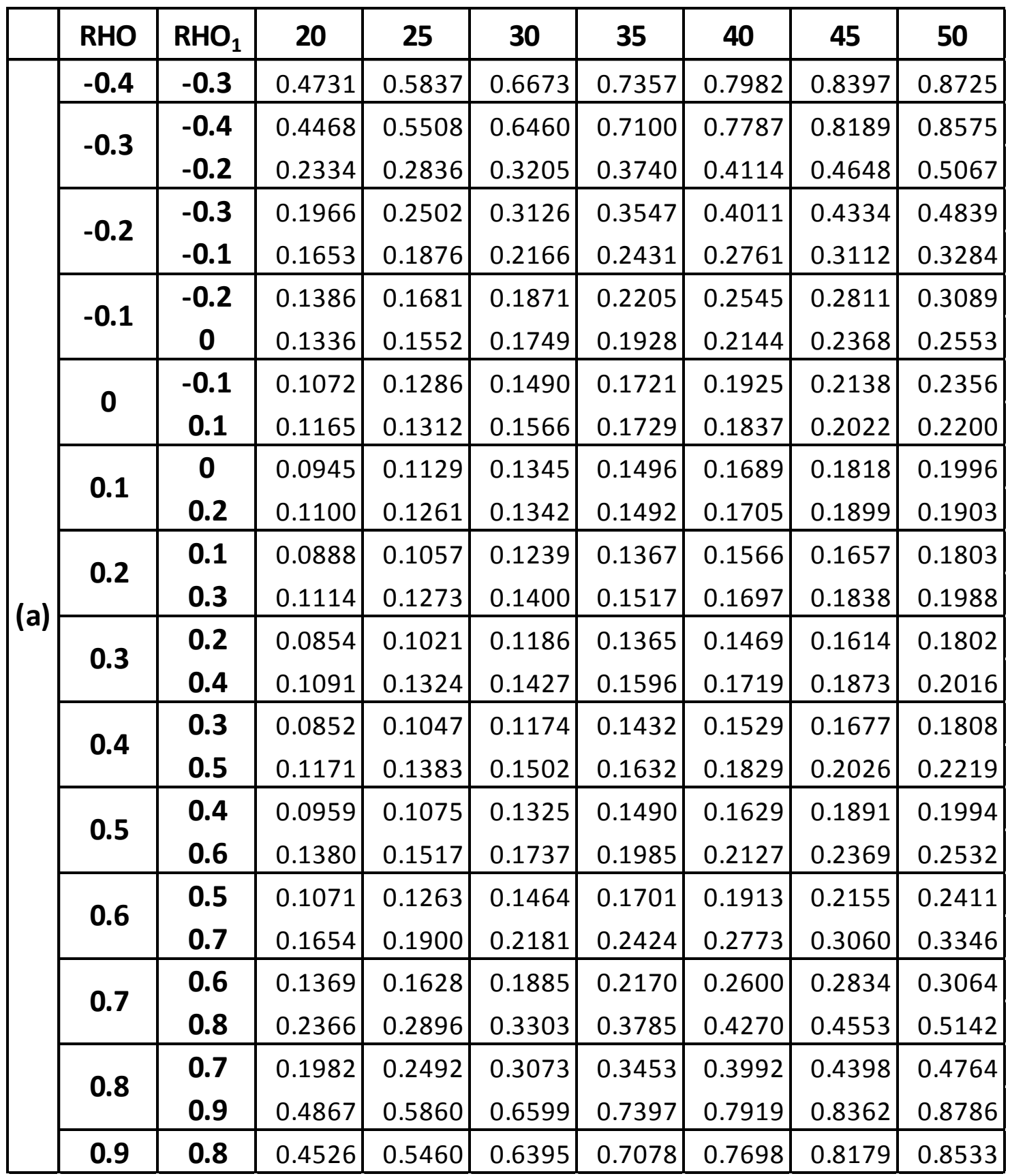

Table 5.9: Comparison Power when $H a: \rho_{1}=\rho_{0} \pm 0.10$ using MLE Trivariate 

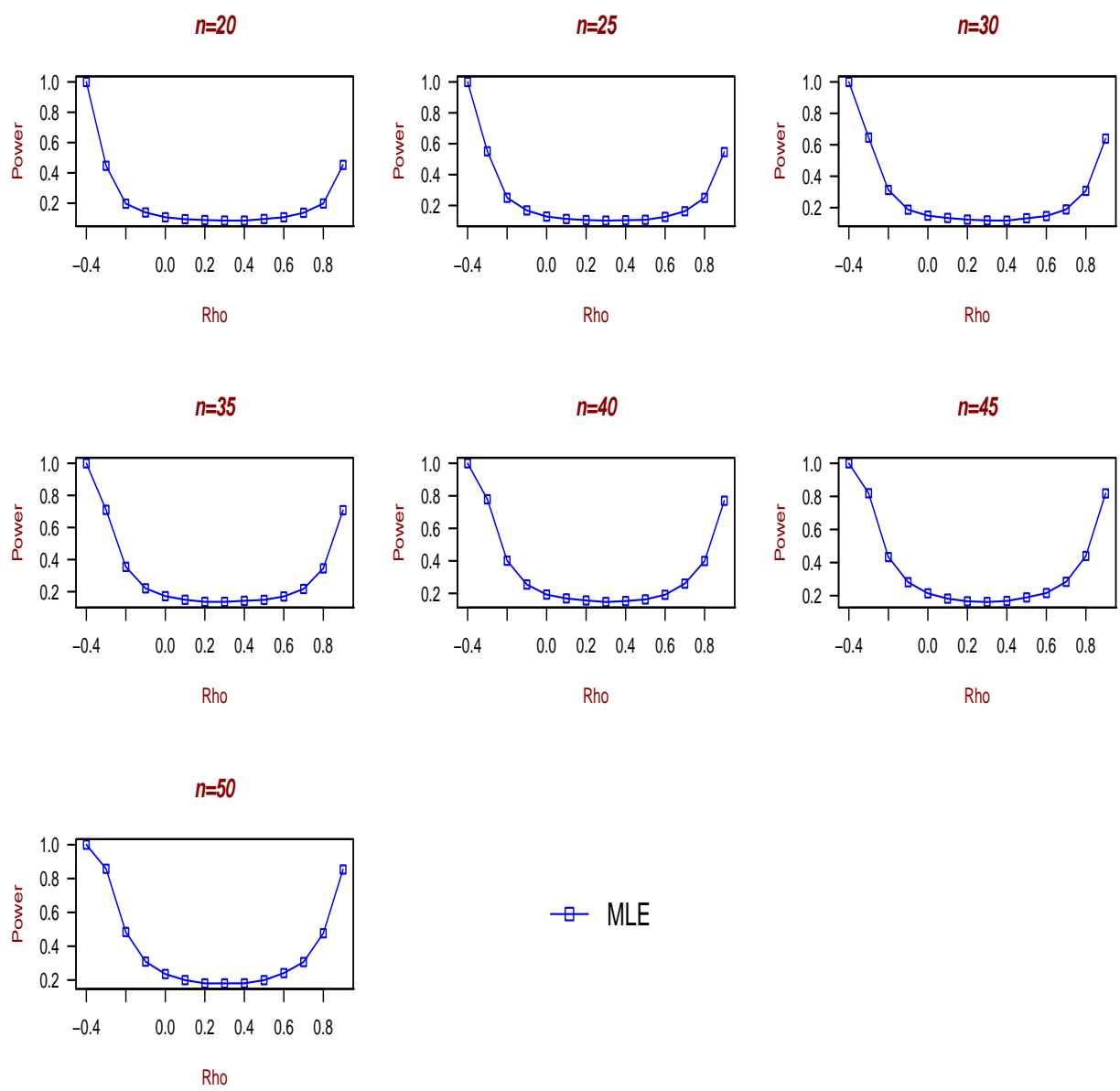

$\rightarrow \cdot M L E$

Figure 5.9: Comparison Power when Ha: $\rho_{1}=\rho_{0}-.10$

In Figure 5.9 we can observed that there the power of testing is higher when $\rho$ approaches to its extreme values and increases as the sample size increases. 

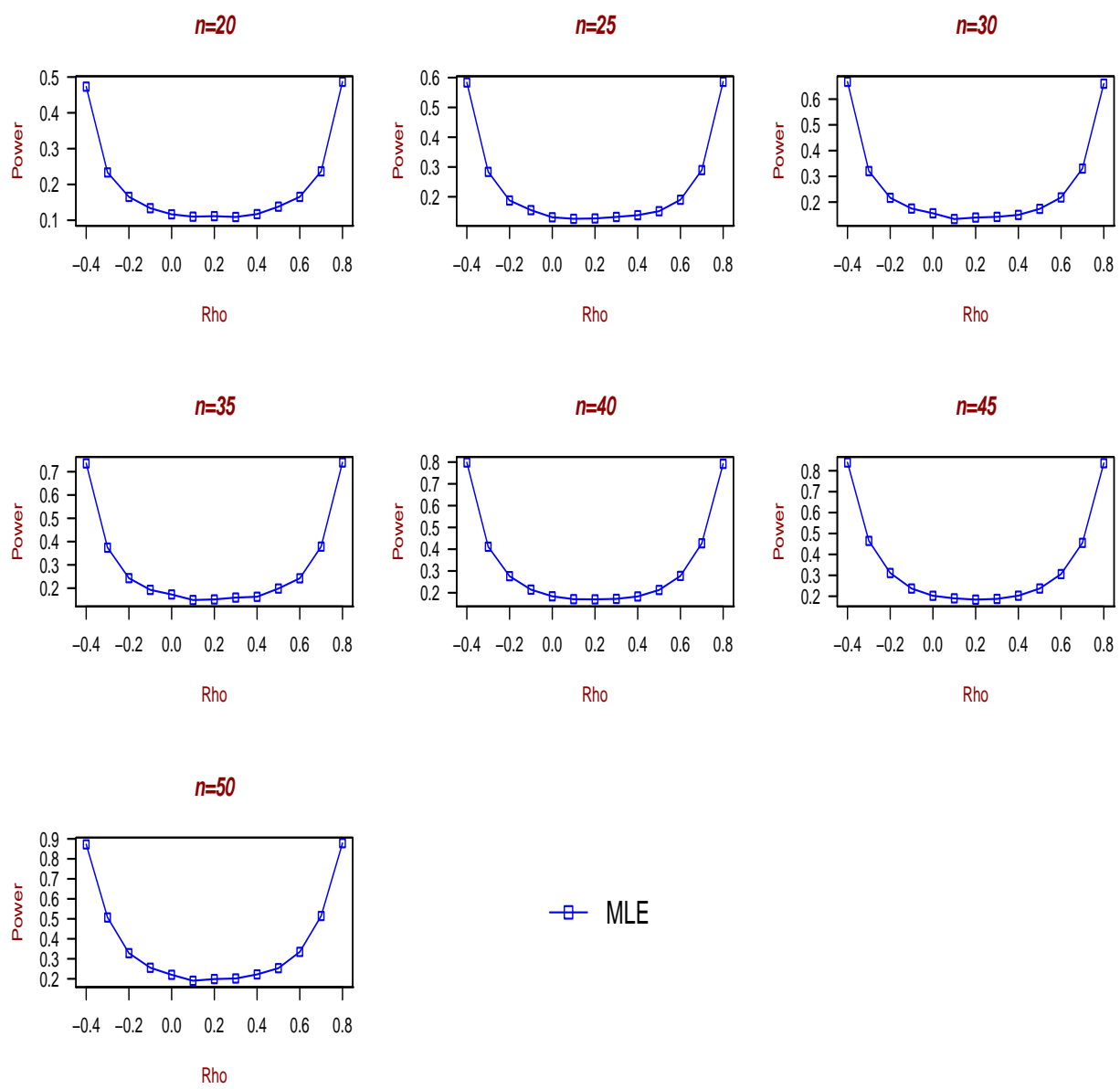

$\rightarrow \cdot M L E$

Figure 5.10: Comparison Power when $\mathrm{Ha}: \rho_{1}=\rho_{0}+.10$

Figure 5.10 occurs the same behaviour as before, that is that the power of testing is higher when $\rho$ approaches to its extreme values and increases as the sample size increases. 


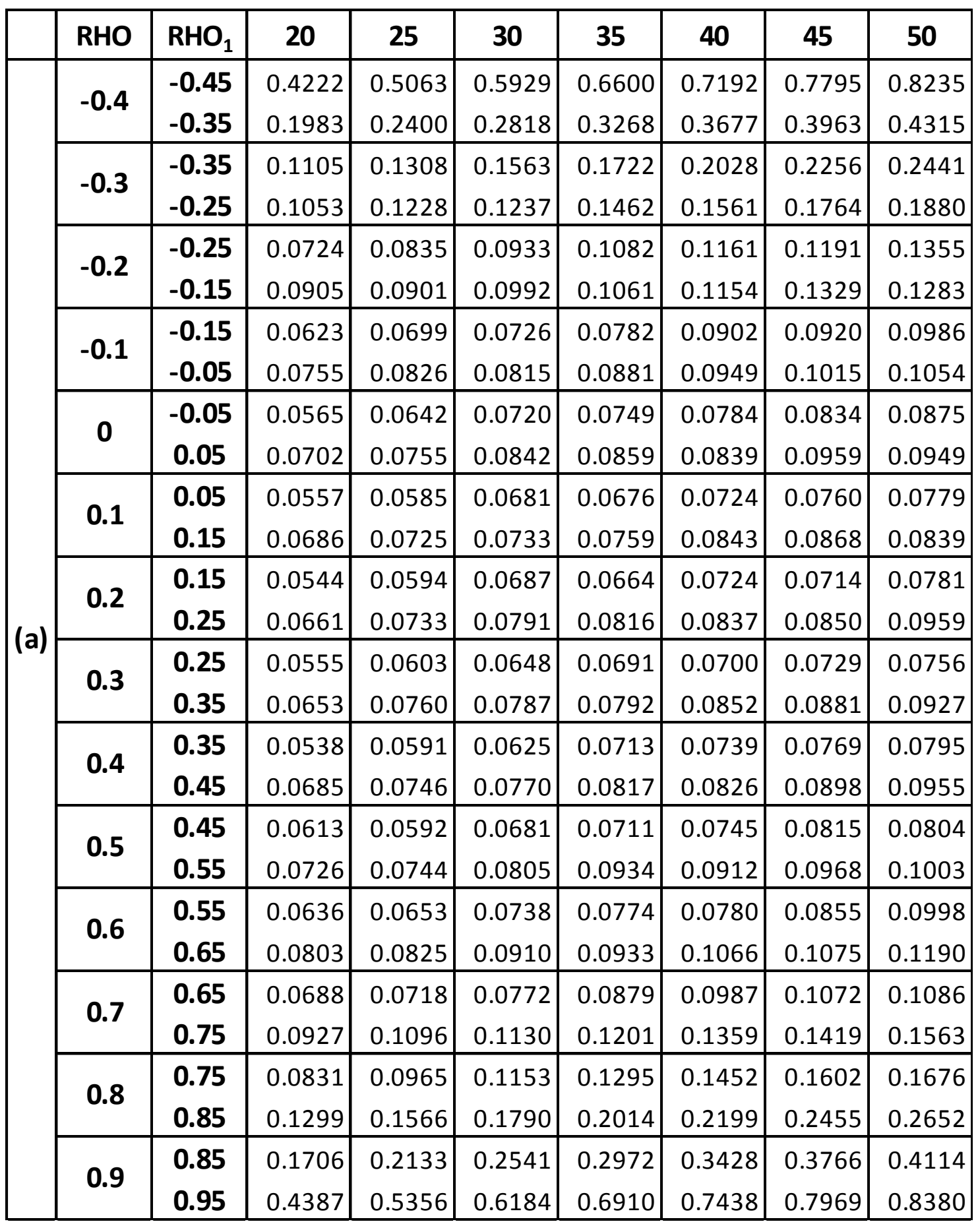

Table 5.10: Comparison Power when $\mathrm{Ha}: \rho=\rho \pm 0.05$ using MLE Trivariate 

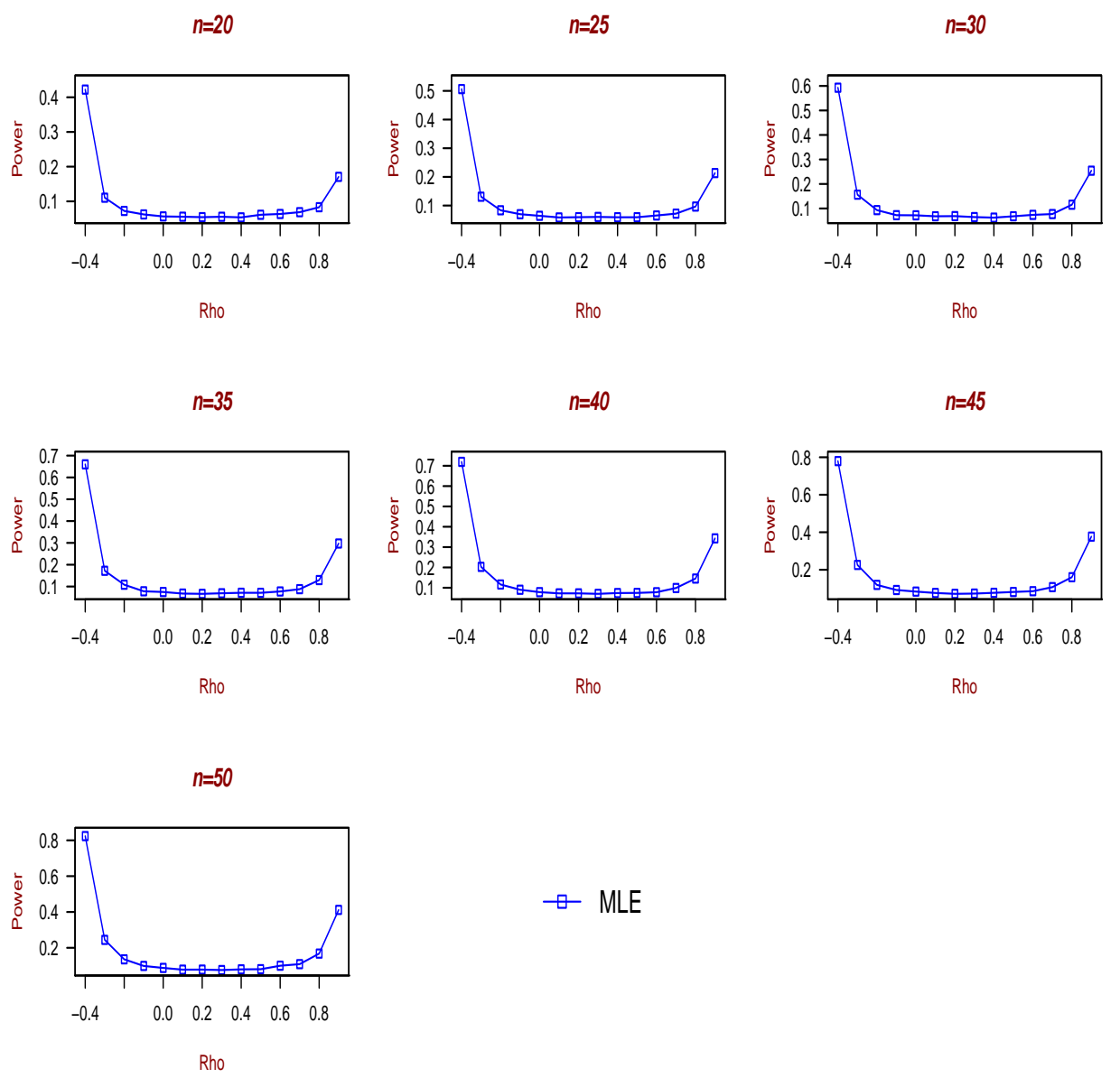

$\rightarrow \cdot M L E$

Figure 5.11: Comparison Power when $H a: \rho_{1}=\rho_{0}-.05$

It can be noticed in Figure 5.11 that the higher power is obtain when $\rho=-0.40$. 

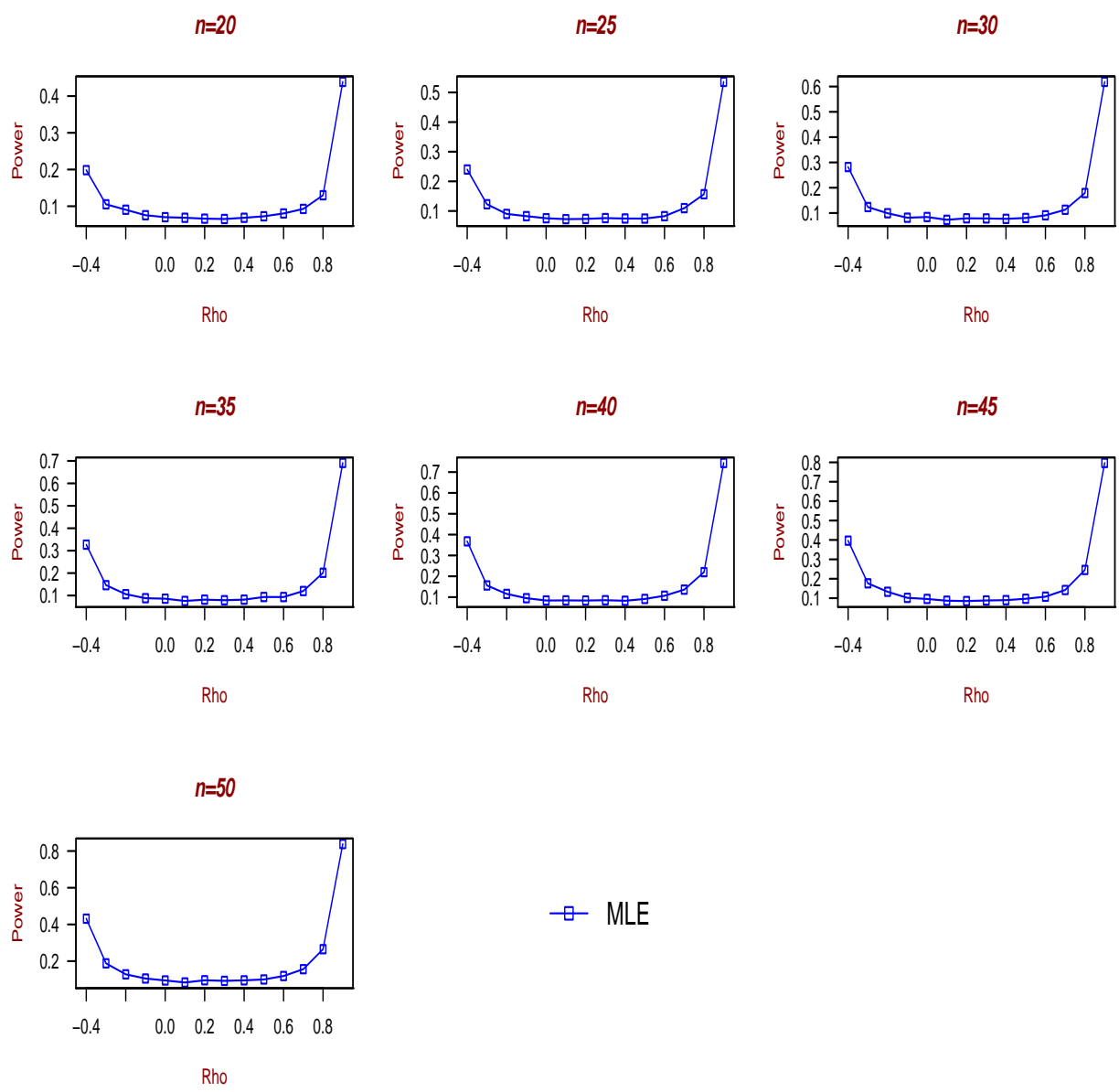

$\rightarrow \cdot M L E$

Figure 5.12: Comparison Power when $\mathrm{Ha}: \rho_{1}=\rho_{0}+.05$

In Figure 5.12 we can see that the higher power is achieved when $\rho=0.9$.

\subsection{Conclusion}

It can be concluded from the numerical study that, the MLE is a good estimator of $\rho$ in both cases, bivariate and trivariate normal distribution with equal variances and convariances. Moreover, the MLE would generally lead to better performance in the following aspects: higher power of testing and narrower confidence intervals, while keeping the coverage probabilities and type I errors close to the desired level. 


\section{BIBLIOGRAPHY}

Y. Sun 2007 and A.C.M. Wong. Interval estimation for the normal correlation coefficient. Statistics And Probability Letters, 77(17):1652 - 1661, 2007. ISSN 0167-7152. doi: http://dx.doi.org/10.1016/j.spl.2007.04.004.

Brian E. Clauser, 2007. The life and labors of francis galton: A review of four recent books about the father of behavioral statistics. Journal of Educational and Behavioral Statistics, 32(4):pp. 440-444, 2007. ISSN 10769986.

R. A. Fisher, 1921. On the probable error of a coefficient of correlation deduced from a small sample. Metron, 1:3-32, 1921.

Ronald Aylmer. Fisher, 2009. Frequency distribution of the values of the correlation coefficient in samples from an indefinitely large population. Biometrika, 10(4): 507-521, 1915.

M. Goria, 1980. On testing the correlation coefficient of a bivariate normal distribution. Metrika, 27(1):189-194, 1980.

Harold Hotelling, 1953. New light on the correlation coefficient and its transforms. Journal of the Royal Statistical Society. Series B (Methodological), 15(2):pp. 193232, 1953. ISSN 00359246.

Elias Masry, 2011. The estimation of the correlation coefficient of bivariate data under dependence: Convergence analysis. Statistics And Probability Letters, 81 (8):1039-1045, 2011.

Jie. Mie. Private communication. 
S. R. Paul, 1988. Estimation of and testing significance for a common correlation coefficient. Communications in Statistics - Theory and Methods, 17(1):pp. 39-53, 1988. ISSN 03610926.

Harold Ruben, 1966. Some new results on the distribution of the sample correlation coefficient. Journal of the Royal Statistical Society. Series $B$ (Methodological), 28(3):pp. 513-525, 1966. ISSN 00359246. URL http: //www. jstor .org/stable/2984445. 